JPL Publication 89-18

\title{
Performance Evaluation of a Six-Axis Generalized Force-Reflecting Teleoperator
}

B. Hannaford

L. Wood

B. Guggisberg

D. McAffee

H. Zak

June 15,1989

\section{N/SN}

National Aeronautics and

Space Administratioñ

Jet Propulsion Laboratory

California institute of Technology

Pasadena, California 


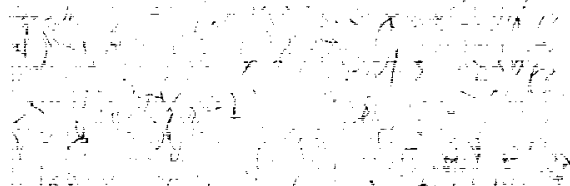

Actor

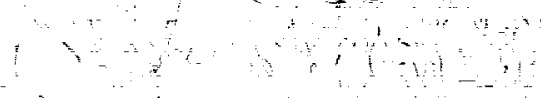

ind

$f=1$

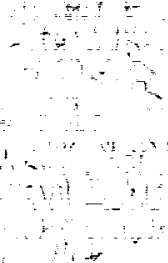

to

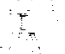

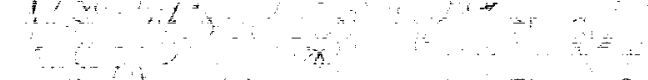

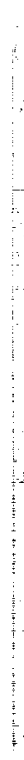


JPL Publication 89-18

\title{
Performance Evaluation of a Six-Axis Generalized Force-Reflecting Teleoperator
}

\author{
B. Hannaford \\ L. Wood \\ B. Guggisberg \\ D. McAffee \\ H. Zak
}

June 15, 1989

\section{N/SA}

National Aeronautics and

Space Administration

Jet Propulsion Laboratory

California Institute of Technology

Pasadena, California 
The research described in this publication was carried out by the Jet Propulsion Laboratory, California Institute of Technology, under a contract with the National Aeronautics and Space Administration.

Reference herein to any specific commercial product, process, or service by trade name, trademark, manufacturer, or otherwise, does not constitute or imply its endorsement by the United States Government or the Jet Propulsion Laboratory, California Institute of Technology. 


\section{ACKNOWLEDGMENT}

The authors would like to thank Antal Bejczy, Paul Lee, Derek Parker, Zoltan Szakaly, and Steven Venema for valuable support and assistance. 


\begin{abstract}
Recent work at JPL in real-time distributed computation and control has culminated in a prototype force-reflecting telemanipulation system having dissimilar master (cable-driven force-reflecting hand controller) and slave (PUMA 560 robot with custom controller), extremely high sampling rate $(1000 \mathrm{~Hz})$, and low loop computation delay (5 $\mathrm{mscc}$. In a series of experiments with this system and five trained test operators covering over 100 hours of teleoperation, we measured performance in a series of generic and application-driven tasks with and without force feedback, and with control shared between teleoperation and local sensor referenced control. Measurements defining task performance include $100-\mathrm{Hz}$ recording of six-axis force/torque information from the slave manipulator wrist, task completion time, and visual observation of predefined task errors.

The tasks consisted of high precision peg-in-hole insertion, electrical connectors, velcro attach-de-attach, and a twist-lock multi-pin connector. Each task was repeated three times under several opcrating conditions: "normal" bilateral telemanipulation, forward position control without force feedback, and shared control. In shared control, orientation was locally servo controlled to comply with applied torques, while translation was under operator control. All performance measures improved as capability was added along a spectrum of capabilities ranging from pure position control through force-reflecting teleoperation and shared control. Performance was maximal for the bare-handed operator.
\end{abstract}




\section{SUMMARY}

An extensive experimental evaluation has been conducted of several modes of teleoperation using an advanced telemanipulation system, a representative sampling of tasks, and five test operators. The results quantified substantial improvements in three measures of task performance as more sophisticated control capabilities were added to the system.

The JPL Enhanced 6-Axis breadboard (ESAB) system (Bejczy \& Szakaly, 1988) is a new telemanipulation architecture featuring the JPL force reflecting hand controller as the input device (master) and a Puma 560 industrial manipulator fitted with the JPL force/torque sensing smart hand as the remote manipulator (slave). Control and coordinate transformation computation is performed by distributing the load among two JPL Universal Motor Control systems, one each on the master and slave sides.

The study developed three measures of performance and applied them to data from multiple repetitions of four classes of tasks by five test operators. The total evaluation represented over 100 hours of experimental teleoperation.

The experiments tested performance of the tasks with 5 modes of teleoperation:

- position control

- position control with visual display of force information

- $\quad$ kinesthetic force feedback

- shared control

- direct manual control

In position control, the slave robot position followed the master and no force/torque information was provided to the operator. In visual display of force, force and torque information was provided to the operator through a computer graphics display. In kinesthetic force feedback, force and torque information was brought back to the operator by applying forces and torques directly to his/her hand through an active joystick. In shared control, force information is simultaneously fed back to the operator, and used by the machine. Machine intelligence and human intelligence cooperate to perform the task.

Direct manual control was achieved by having the operators perform the task directly without the remote manipulation system involved. This experimental condition provided an absolute benchmark independent of any particular telemanipulation technology. The other modes were provided by the control software of the ESAB.

The tasks consisted of a mix of basic tasks designed to measure technical capabilities as well as to illustrate suitability for applications. The four tasks were: 


\section{Attach and detach Velcro fasteners}

Velcro is commonly used in space vehicles for keeping parts fixed in the absence of gravity. Its hook and loop fastening system has nonlinear mechanical properties which challenge manipulation capabilities.

\section{Insert a peg into a machined hole}

Peg-in-hole is the classic task in robotic interaction with a mechanical environment. It is a test of the robot's ability to achieve ultra-precision positioning in spite of nonlinear mechanics and imprecise knowledge. The holes and peg in this experiment were machined to tolerances common in automotive bearing practice.

\section{Manipulate standard electrical connectors}

This task is similar to the peg-in-hole task except that the components were standard offthe-shelf electrical signal and power connectors: $120 \mathrm{~V}$ chassis power cord, $1 / 4$ " headphone jack, 25-pin computer connector.

\section{Connect a bayonet style multi-pin connector}

This connector was more difficult than the others because it required a rolling motion to make/break the physical and electrical connection.

Performance was evaluated with three measures: completion time, error rate, and sum-ofsquared-force (SOSF). All of these measures decrease with "better" performance of a given task. The first is a purely economic measure applicable to predicting the cost of completing a given operation. The last two are quality measures applicable to assessment of the degree of risk with which a task can be performed.

\section{RESULTS}

Some condensed results of the study are presented in Figures $\mathrm{i}$ and ii. Task completion time and the SOSF force performance measure were computed for the peg-in-hole and the electrical connectors tasks. In these computations, data from nine different hole geometries (in the case of the peg-in-hole task) and separately, the three electrical connector types (in the case of the electrical connector task) were averaged over the five test operators to come up with overall performance figures for the five control modes described above.

For the peg-in-hole task, both completion time and force dropped dramatically as the control capability was increased from pure position control to the exquisitely sensitive control of the bare human hand. For the electrical connectors, completion time was substantially reduced only with shared control, but the force generated to complete the tasks dropped significantly even with only visual display of the force information. 


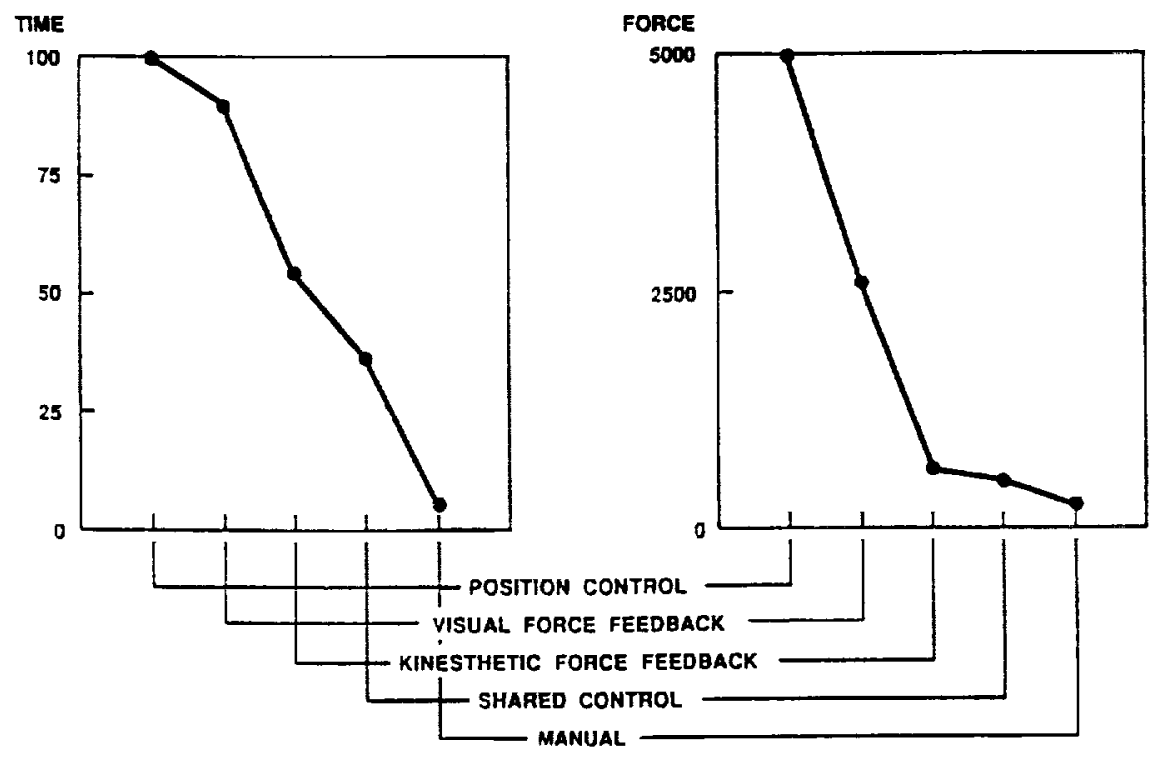

Figure i

Completion time and force performance for the peg-in-hole task. The data are averaged over 27 repetitions by five test operators in each mode.

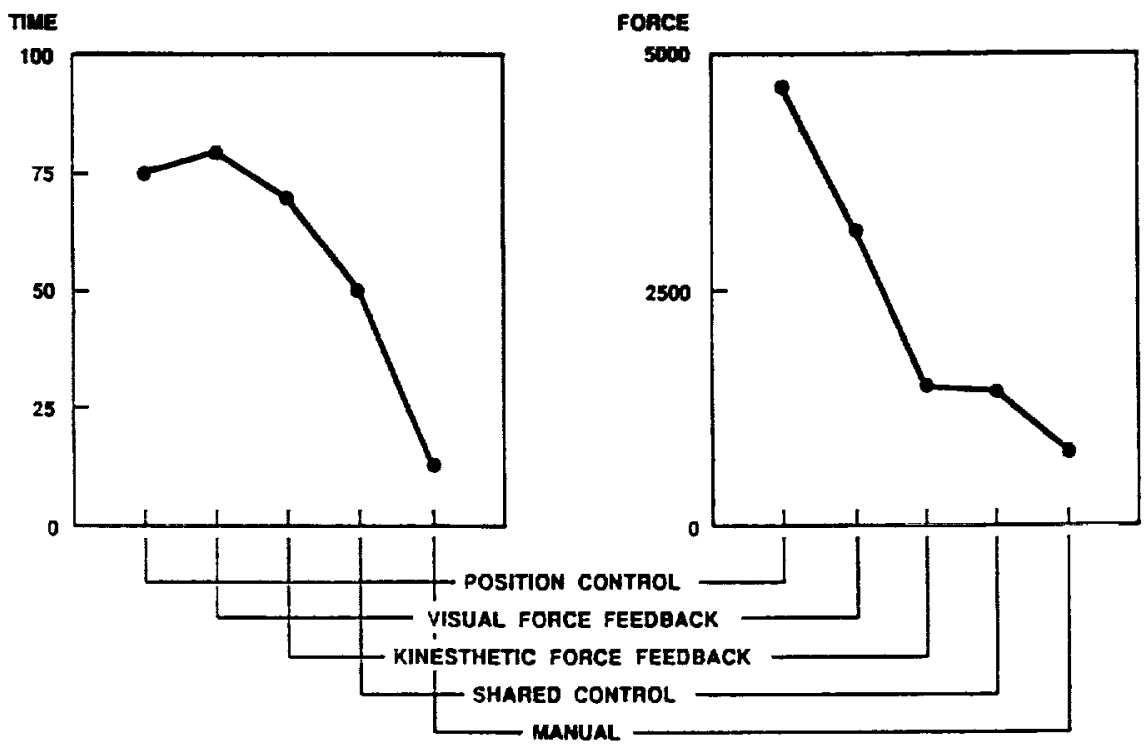

Figure ii

Completion time and force performance for the electrical connectors task. The data are averaged over 3 repetitions of insertion and extraction of three standard electrical connectors by five test operators. 


\section{CONCLUSIONS}

This study has quantified the distance yet to be covered before teleoperators will have the same dexterity as the bare human hand. We have measured task performance in three dimensions: completion time, force performance, and error rate. All of these measures improved as capabilities were added to the manipulation system although the improvement varied as a function of task. The telerobotic system we tested has an extraordinary number of control configurations (at least one million!). Only two of these forms of shared control were tested and the resulting improved task performance has shown significant potential for the technology. The performance of the unaided human was measured to serve as an upper bound on manipulation performance.

The performance of the unaided operator should be carefully differentiated from the case of an EVA astronaut. EVA task performance is reduced due to various factors such as the mechanics of the suit and glove, the elimination of tactile sensation, and space physiological effects. For mission planning purposes, pre-EVA time spent donning and removing the suit as well as prebreathing, etc. must be amortized over the available work time. The exquisite level of dexterity achieved in these experiments by natural human manipulation is a goal to which both telerobot and space suit designers can strive.

Future work will need to address additional constraints and technologies foreseen in space applications. Experiments currently under way are addressing the effects of time delays interposed between master and slave, and the unique characteristics of dual arm telemanipulation. 


\section{TABLE OF CONTENTS}

Acknowledgment $\ldots \ldots \ldots \ldots \ldots \ldots \ldots \ldots \ldots \ldots \ldots$ iii

Abstract $\quad \ldots \ldots \ldots \ldots \ldots \ldots \ldots \ldots \ldots \ldots \ldots$ iv

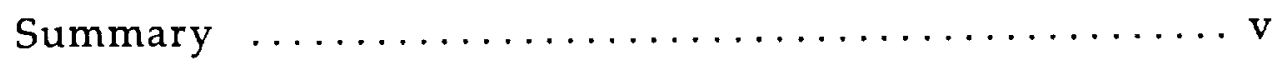

Introduction $\ldots \ldots \ldots \ldots \ldots \ldots \ldots \ldots \ldots \ldots \ldots \ldots \ldots \ldots \ldots$

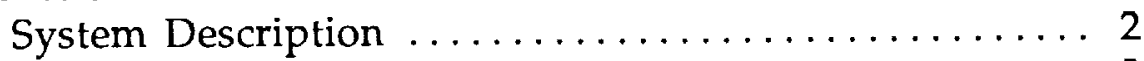

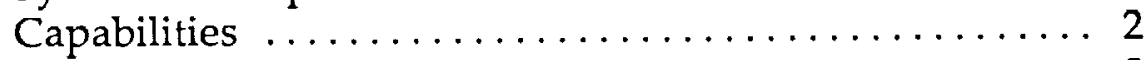

Control Station $\ldots \ldots \ldots \ldots \ldots \ldots \ldots \ldots \ldots \ldots, 8$

Experiment Goals ...................... 8

Methods ................................... 9

Experiment Design $\ldots \ldots \ldots \ldots \ldots \ldots \ldots \ldots \ldots, \ldots$

Analysis and Performance Measures ............. 19

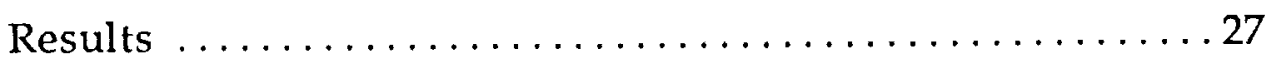

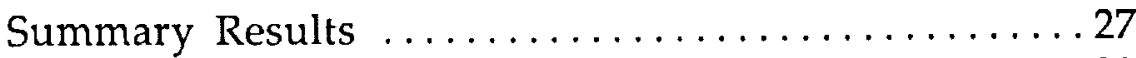

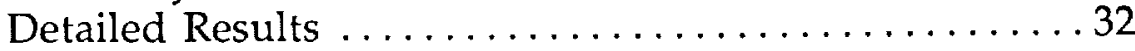

Preliminary Results: Additional Control Modes ...... 45

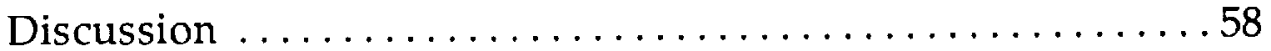

System and Control Modes $\ldots \ldots \ldots \ldots \ldots \ldots \ldots \ldots$

Mechanics and Stability $\ldots \ldots \ldots \ldots \ldots \ldots \ldots \ldots . \ldots \ldots$

Tasks ....................................... 59

Subjects ............................60 60

Conclusions and Recommendations $\ldots \ldots \ldots \ldots \ldots 60$

Future Work ........................61

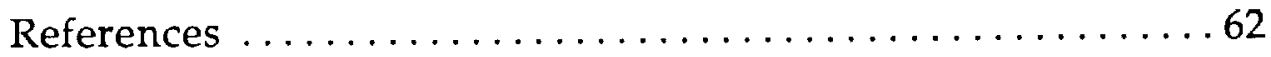

Appendix A: Modular Task Board For Telemanipulation Experiments

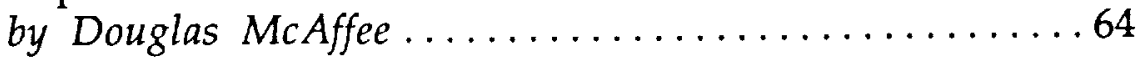

Appendix B: Task Board Modifications and Improvements by Brent Guggisberg ........................ 92

Appendix C: Task Sequence Descriptions $\ldots \ldots \ldots \ldots \ldots 112$

Appendix D: Operator Skill Testing and Prediction

by Haya Zak ............................. 114

Appendix E: Typical Force/Torque Records for

Additional Tasks 
$-$

- 


\section{INTRODUCTION}

Teleoperation is expected to perform a wide variety of tasks in future space operations. Tasks such as space-station construction will create huge demands for extra-vehicular activity (EVA), which will far exceed available astronaut time. Of course, a major benefit of teleoperation is enhanced human safety: the operator is physically removed from the actual worksite and the sources of danger, located instead at a control station on earth or in a "shirtsleeve" environment on orbit. The price paid for the advantages of teleoperation is usually expressed in reduced performance. Thus it is important to devote attention to the quantitive evaluation of telerobotic technology in order to assess its benefits in terms of mission productivity.

In order to pursue the goal of a realistic sense of presence at the worksite, many manipulator designs, starting with Goertz (1954), have incorporated force feedback capabilities. For a good review see Goertz (1964). As a result, many of the studies attempting to quantify the performance of various teleoperator designs have concentrated on the question "Can force/torque feedback improve teleoperation performance?"

Kugath (1972) studied the effects of a compliant manipulation arm and force feedback on manipulation of an inertial load. Tasks performed included the capture of a $400 \mathrm{lb}$ penclulum and manipulation of a $650 \mathrm{lb}$ mass through a maze on a flat-floor air-bearing table. Results showed force feedback had a large effect on task completion time and error rate (i.e., hitting the wall) for the maze task.

Hill and Salisbury (1977) evaluated three master-slave teleoperators (the Ames Exoskeletal Master Slave, MA11 \& MA23 by Flatau et al. 1972) with an instrumented task board which emphasized the peg-in-hole task. Their results documented task completion time for the different arms as a function of peg tolerance and showed improved performance when force feedback was present. For a given peg clearance, task completion time varied linearly with distance. They also reported bare-handed operator performance. The pegs used ranged in size from 1 inch to 1.996 inches and were to be placed in a two-inch hole. Hill's later work (1979) developed a model of teleoperator task completion time from industrial time and motion models.

Other studies include those by Bejczy and Handlykken (1981), who investigated the effects of varying force-feedback gain in the novel generalized bilateral teleoperator architecture in which the force-reflecting master hand controller (Bejczy \& Salisbury 1983) is kinematically optimized for man-machine coupling, and real-time computation is provided for coordinate transformations between the master and slave joint spaces.

Recent evaluation studies (Draper et al. 1987) have emphasized on broadening the base of measurements against which task performance can be judged. Besides task completion time, useful measures included number of task errors, peak force and variance in force (see also Hannaford 1987). Although ANOVA did not show a significant completion time improvement due to force feedback, the other measures did indicating that force feedback allowed the task to be performed with higher-quality results if not at a faster rate. 


\section{SYSTEM DESCRIPTION}

The JPL teleoperation laboratory has recently developed a unique telemanipulation system featuring advanced modes of shared- and supervisory-control-based remote manipulation (Szakalay, Kim, \& Bejczy 1989). The Enhanced Six-Axis Breadboard (ESAB) teleoperation system consists of the JPL-Stanford Force Reflecting Hand Controller (recently refurbished and upgraded from the original [Bejczy \& Salisbury 1981] design), a Puma 560 manipulator, and the JPL Puma Smart Hand (Fiorini 1988) (see Figure 1a and 1b).

The JPL Puma Smart Hand is a single-degree-of-freedom gripping mechanism mounted in series with a six-axis force-torque sensor. A local microprocessor performs gripping control, force-torque sensor processing, and communication functions. These devices are integrated and controlled with two JPL universal-control computer systems (Bejczy \& Szakaly 1987), which perform all coordinate system transformations, master and slave DC motor control, and operator interface functions. No part of the Unimation Puma control system is used.

The computation and control system electronics are completely housed in two specially modified multibus card cages. The card cages contain NS 32000 family computer boards, power supplies, and power amplifiers for the master and slave. Master and slave electronics card cages are connected by a parallel cable for bidirectional exchange of position and force data.

The system contains a total of five NS 32016 processors dedicated to forward and inverse kinematics computations for the master and slave manipulators. Proccssors communicate locally via shared memory, and between master and slave sicles through the parallel cable (Figure 2).

\section{CAPABILITIES}

The ESAB system can be configured by the user in a wide variety of ways. All control modes and gains can be independently selected for each task-space axis. Motion Control modes include position control, rate control, and "none." A "bead diagram" (Figure 3) can be used to express the permissible control modes for each axis. In this diagram, a valid control mode can be selected by stringing one bead from each column without crossing a horizontal bar. The current system thus permits 10 possible control modes per axis. The total number of control modes is thus a mind boggling $10^{6}$ modes! In addition, for each option column, one or more parameter value must be specified. The ESAB thus provides an almost infinite set of control possibilities.

The Position-Control mode servo controls the slave position and orientation to match the master's. An indexing function allows slave excursions beyond the 1-cubic-foot work volume of the Force-Reflecting Hand Controller (FRHC), and allows the operator to work at any task site and orientation from his or her own most comfortable position. 


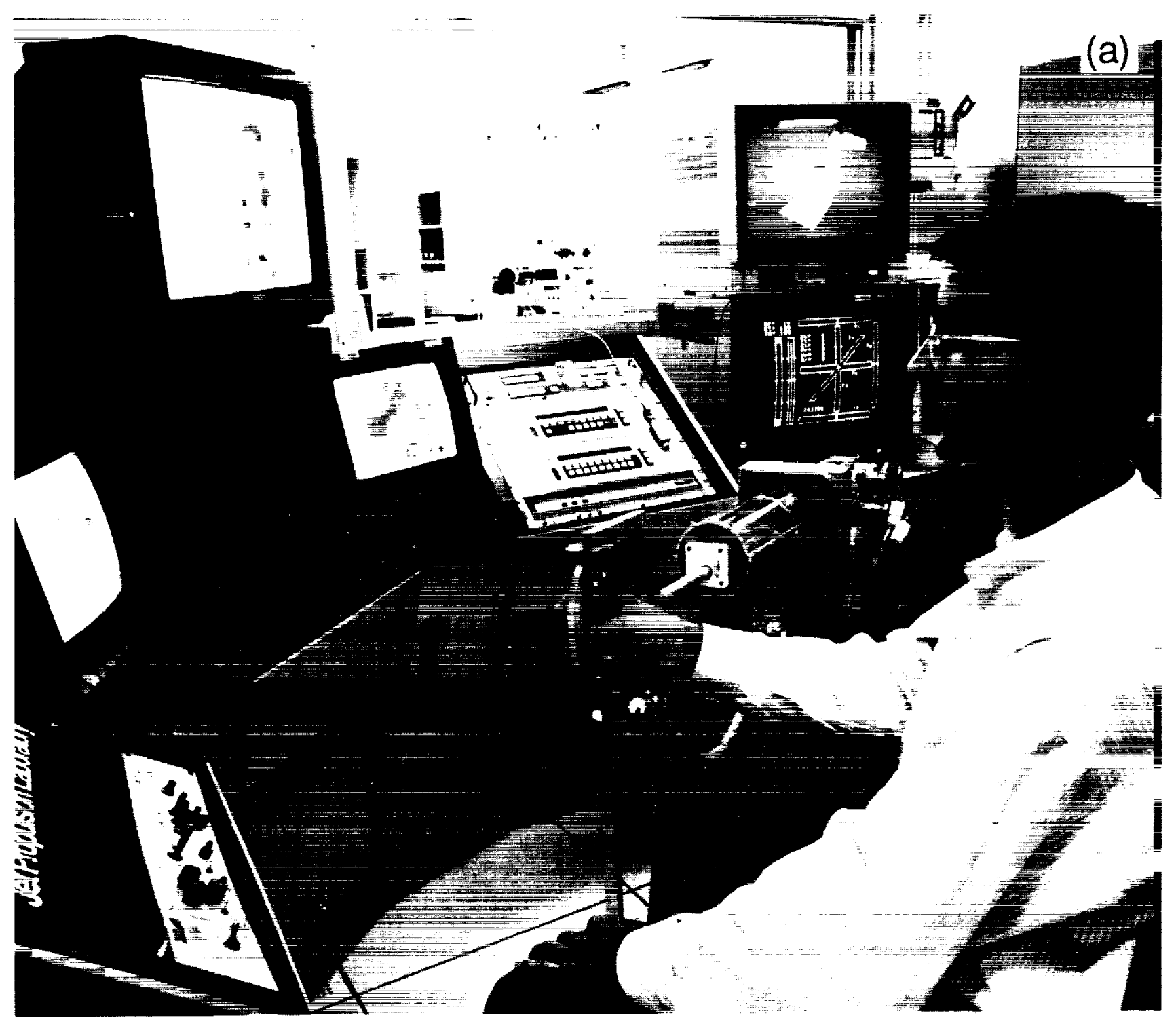

Figure 1(a) JPL Enhanced Six-Axis Breadboard System seen from operator control station. Operator holds six-degree-of-freedom force-reflecting hand controller through which position commands are send to the PUMA 560 industrial manipulator (background). Forces sensed by the manipulator hand are transmitted to the operator's hand via the hand controller. Coordinate transformations are performed by a special-purpose computing architecture (not shown). Operator views task board with the three task monitors (left). A curtain blocks direct viewing during training and experimentation. Also shown are graphics displays: force/torque information (bottom right) and real-time solid shaded animation (top right). 

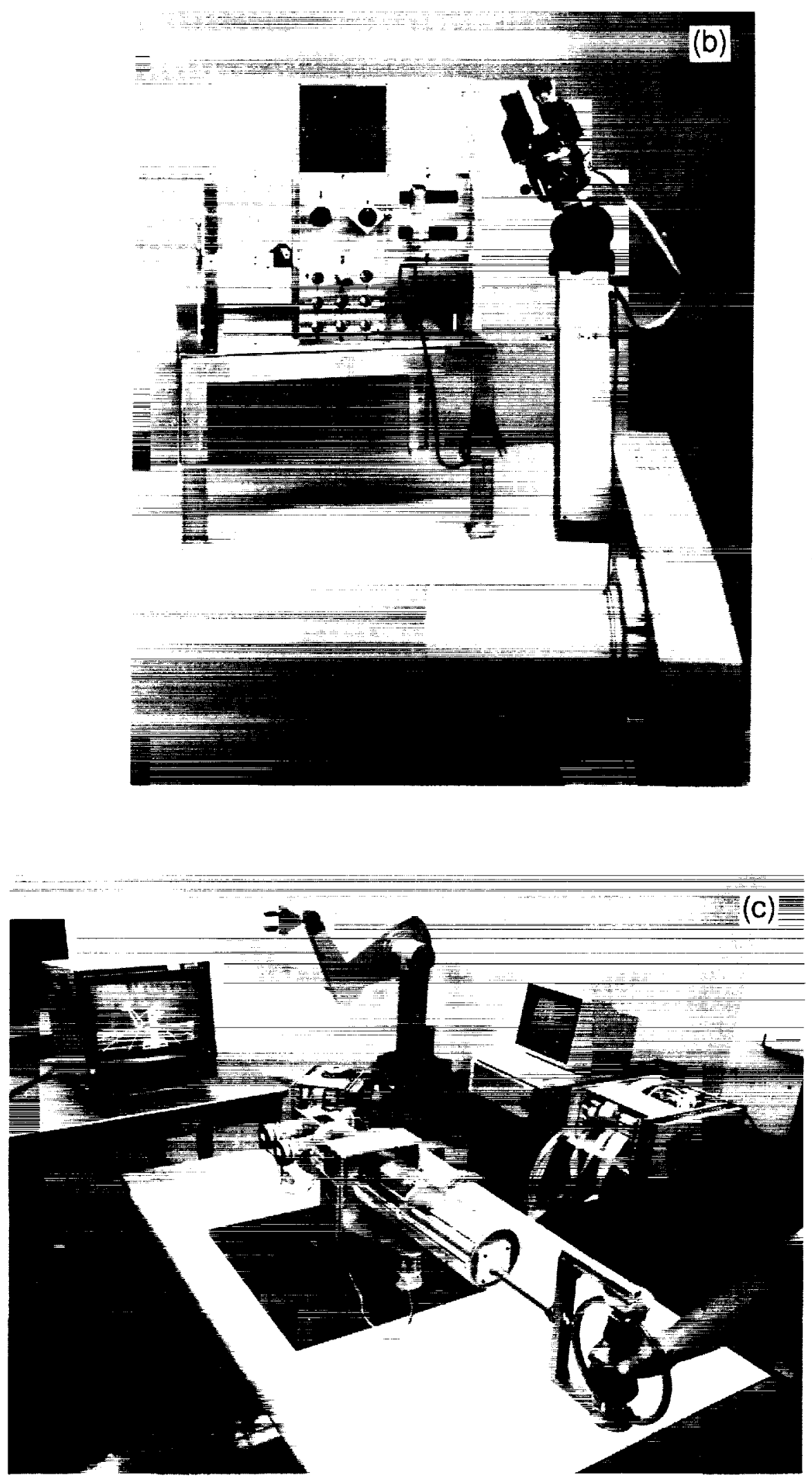

Figure 1(b) and (c). System components consist of (b) the Puma 560 manipulator, JPL Smart Hand, and modular task board; and (c) the force-reflecting hand controller (foreground), control electronics (bottom right), and predictive graphics display. The graphics display was not used in these experiments. 


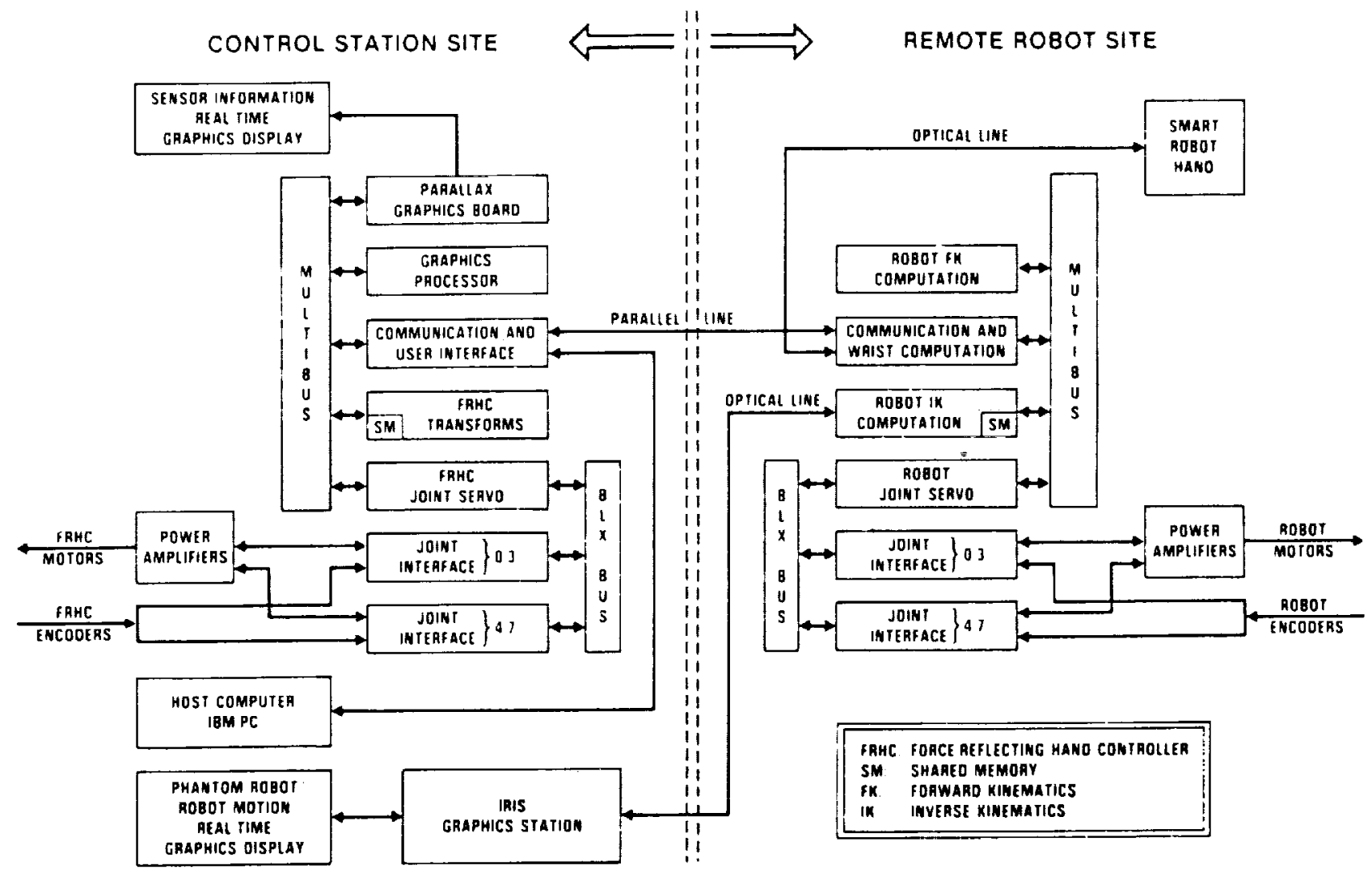

Figure 2. System block diagram of high speed dedicated multi-processor system which performed coordinate transformations and control of master hand controller and slave robot manipulator. 


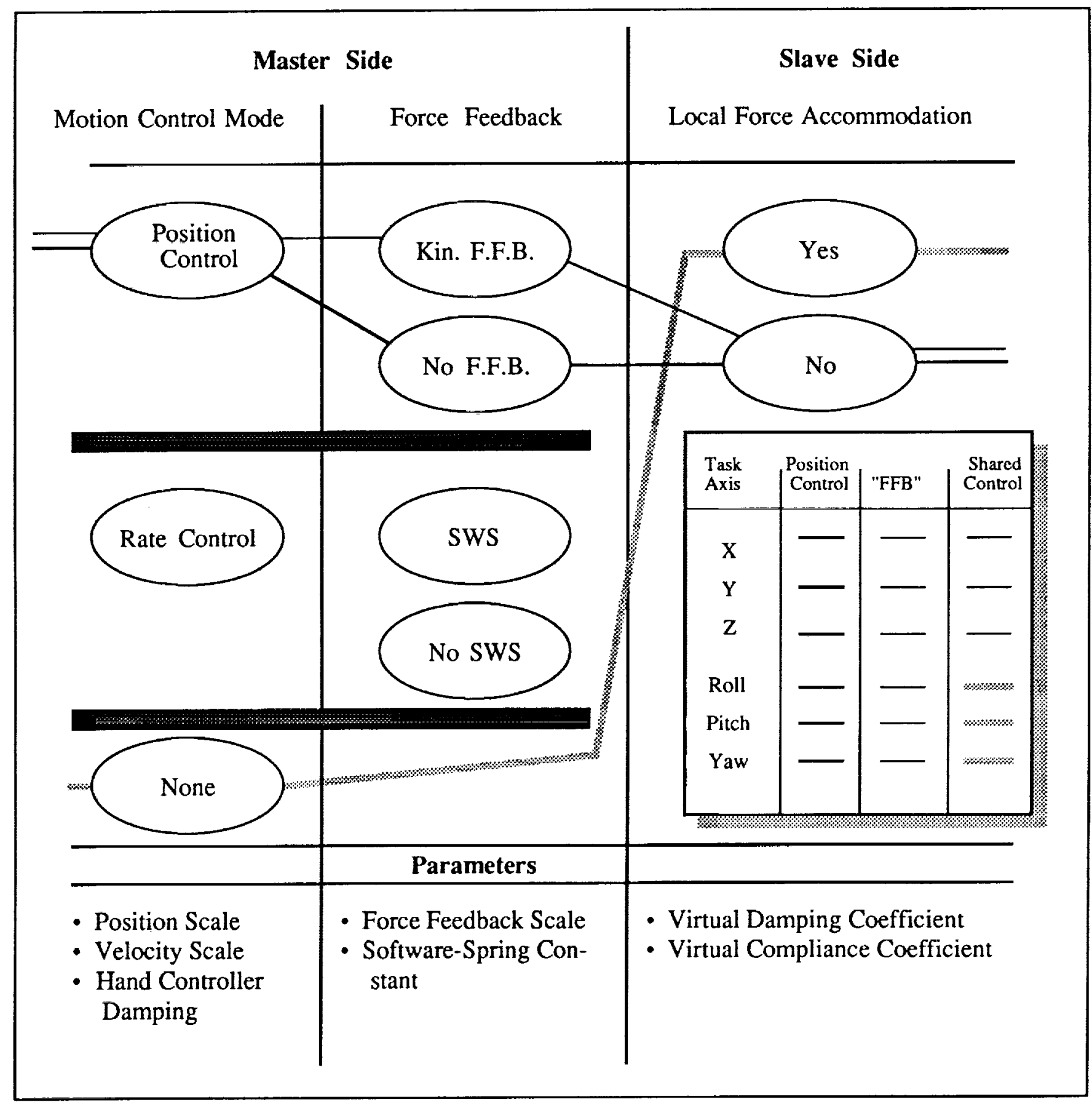

Figure 3. Control of each axis of the six axis telemanipulation system can be specified by conceptually "threading a string of beads" from left to right without crossing any heavy lines. Within each column, one or more parameter values may be chosen as appropriate. Key: "Kin. F.F.B.," Kinesthetic force feedback, "SWS," Software Spring - a restoring force which returns the joystick to the position corresponding to zero velocity. Inset shows typical control modes selected for each axis for the experiments in this study. 
Force Feedback can be generated by the hand controller to serve two functions: reflection of task forces and application of a return force to center the hand controller when released (this function is referred to as "software spring" or SWS). Force reflection is optionally used with position control and SWS with rate control.

Rate Control sets slave endpoint velocity or angular velocity in task space based on the displacement of the FRHC.

Position and rate modes exist solely on the master side - the slave receives the same incremental position commands in either case. In contrast, Force-Accommodation mode resides at the slave side. In this mode, in selected degrees of freedom the slave computer generates position setpoints based on integration of the sensed force or torque in that direction. The effect of this integrated force feedback is to produce a viscous mechanical impedance at the end effector. This is equivalent to the generalized damper of Whitney (1985). (Recent work has extended this mode to include true compliance, e.g., the manipulator emulates a spring and damper in the selected axes.) Force-accommodation mode provides a local control capability for complex manipulation tasks such as the pegin-hole task.

Because of the local force accommodation capability, several forms of "shared control" (in which both manual and automatic control coexist) are possible. For example, control can be allocated in each task-space degree of freedom in a manner similar to Hybrid Control (Raibert \& Craig 1981) between manual and automatic (local) control. Alternatively, control can be shared even in the same axis. In this study, "Shared Force Accommodation" shall describe a mode in which the operator's commands and the local force accommodation signal are summed in task-space orientation axes and position control with force feedback used in translation axes. "Hybrid Force Accommodation" shall describe a mode in which orientation was solely under control of local force accommodation and translation under position control by the operator.

In this study, five control modes plus direct human task performance were experimentally tested. These are:

1. Position control without force feedback

2. Position control with visual force and torque display

3. Position control with kinesthetic force feedback

4. Shared Force Accommodation

5. Hybrid Force Accommodation

6. Direct human operation

The same performance measures were computed for all six modes of operation. Direct human operation was included to provide a measure of task difficulty which is independent of the manipulation system we used. Presumably the tasks used in this study can be related 
to others by comparing human performance figures. The human performance figures also provide a goal point for telemanipulator system performance. Direct human operation bears the same relationship to EVA. Direct human operation represents the performance point toward which space-glove designers can aim.

\section{CONTROL STATION}

The master side of the system is installed in a separate control station without a direct view of the robot work area. Three television cameras provide top, upper-left rear, and right rear views of the task board. The two rear-view cameras could be remotely controlled for focus and zoom, but fixed views were used for all of the experimental tasks.

\section{EXPERIMENT GOALS}

This study is part of a longer-term effort to quantitatively evaluate a snapshot of present telemanipulation technology to expose areas in which improvements are needed for specific real-world applications. An additional goal was to explore the possibilities for the evaluation or preselection of operators of telerobots. As a first step in this direction, we looked at some well-known motor control tests to assess their predictive value for teleoperation performance.

The overall experimental approach was to design a preliminary experiment which looked at a relatively large number of independent variables. The results of this study are being used to guide current work in which focused experiments are performed to obtain statistically powerful results.

The tasks used for teleoperator evaluation fall naturally into two classes: generic tasks and application tasks. Generic tasks are idealized, simplified tasks which are designed to test specific telemanipulation capabilities. Application tasks are designed as much as possible to mimic real-world uses for teleoperation. Evaluations based on generic tasks illustrate the capabilities of existing telerobotic technology, while application tasks guide the technology in the direction of greatest payoff. 


\section{METHODS}

\section{EXPERIMENT DESIGN}

\section{Study Variables}

The experiment design varied control mode, task, and subject. The dependent measures were task completion time, sum of squared forces (SOSF), and number of errors. Three repetitions of each subtask were performed by each subject in each of the control modes. All subtasks were performed in random sequence to form one repetition (randomization without replacement). All subjects performed all repetitions of a given control mode before the next mode was tested.

Robot sensing and control data were recorded by a Unix-based laboratory computer system through its real-time parallel port. This parallel port is connected to a port on the universal controller which can send any of the robot control parameters at the servo rate of $1 \mathrm{kHz}$. We recorded the six axes of force and torque information, as well as gripper opening position and gripping force sensed by the two fingers. A typical record of this raw data forms eight plots. Figure 4 shows one repetition of the peg-in-hole task. The information is serially multiplexed in a packet of 10 bytes for a net sampling rate of $100 \mathrm{~Hz}$ per channel. The complete data vector is described in Table 1. One of the nine data bytes is transferred every millisecond followed by a synchronization byte. Task information such as completion time and task performance strategy can be computed from the time records of forces and torques used in performing the task (Hannaford 1987). Data recording began and ended when the experimenter pressed a mouse button on the laboratory computer system.

We measured force/torque information of the direct human operation by mounting the task modules on a force/torque sensor removed from the robot and attached to a stable base. The task board surface was mounted at the center of the force-sensing coordinate frame. This had the effect of reversing measured forces in the $y$ and $z$ directions relative to the robot tasks. The SOSF (see below) force performance measure ignores this difference. 

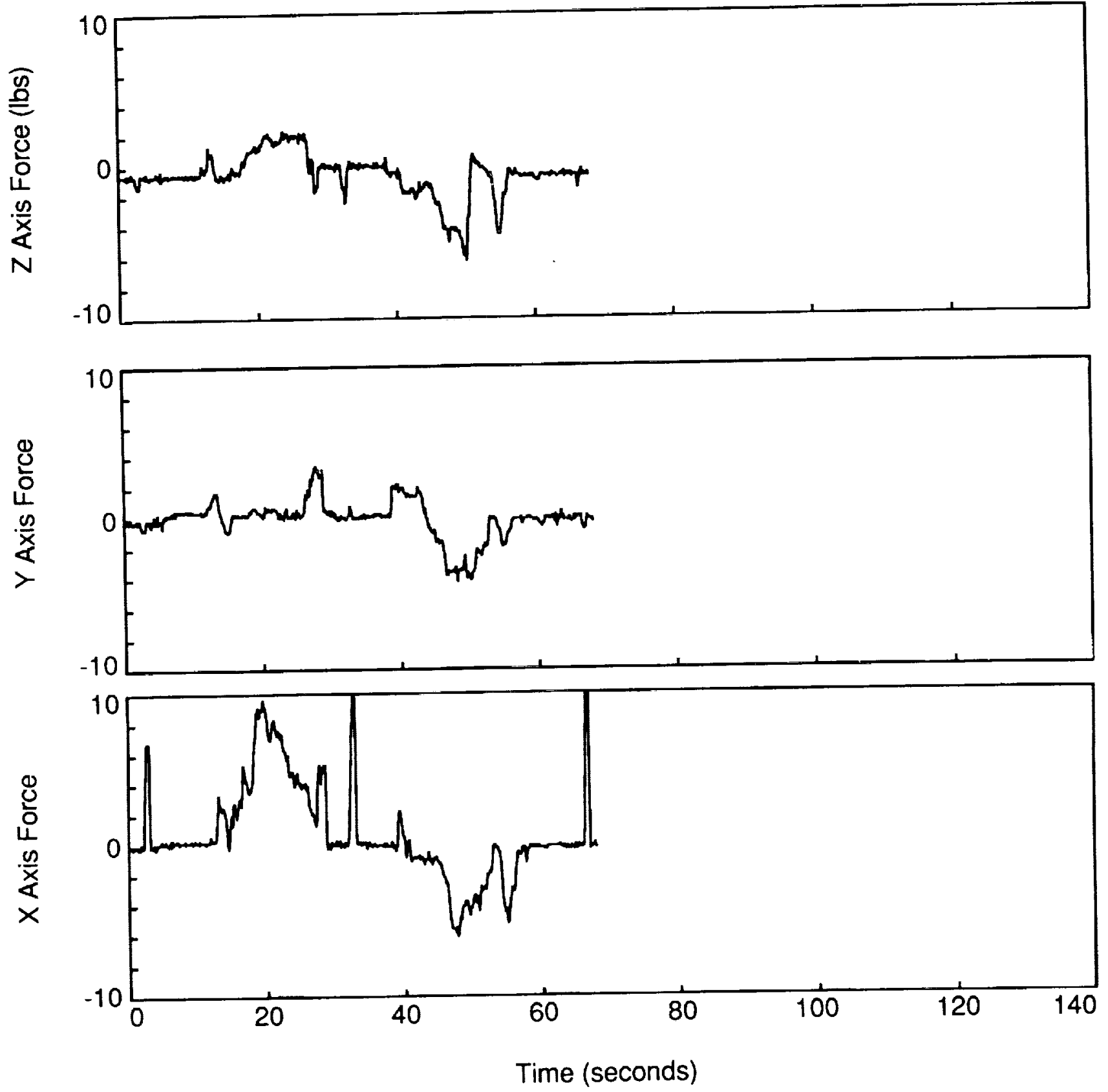

Figure 4. $x, y$, and $z$ Forces during teleoperation. Raw data acquired from a single repetition of the peg-in-hole experiment (continued in Figures 5 and 6). $x, y$, and $z$ axis forces measured in the robot-hand reference frame during insertion ( $t=16$ to $t=24$ seconds) and extraction ( $t=$ 40 to $t=60$ seconds) of the peg. Spikes represent taps made intentionally by the operator to provide reference benchmarks in the force signal. 

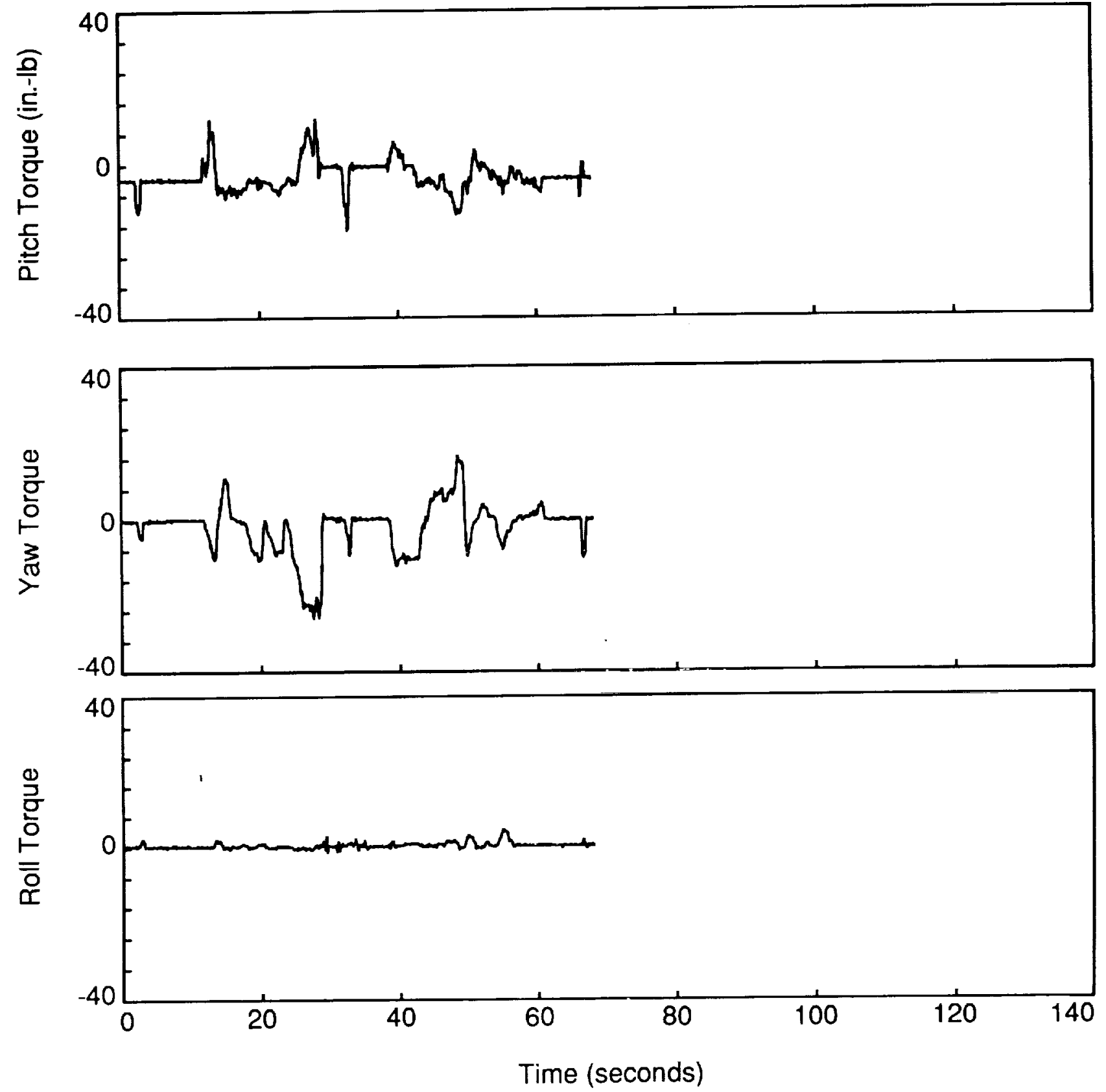

Figure 5. Pitch, yaw and roll torques during the peg-in-hole task. Recording is from the same repetition as in Figure 4. 

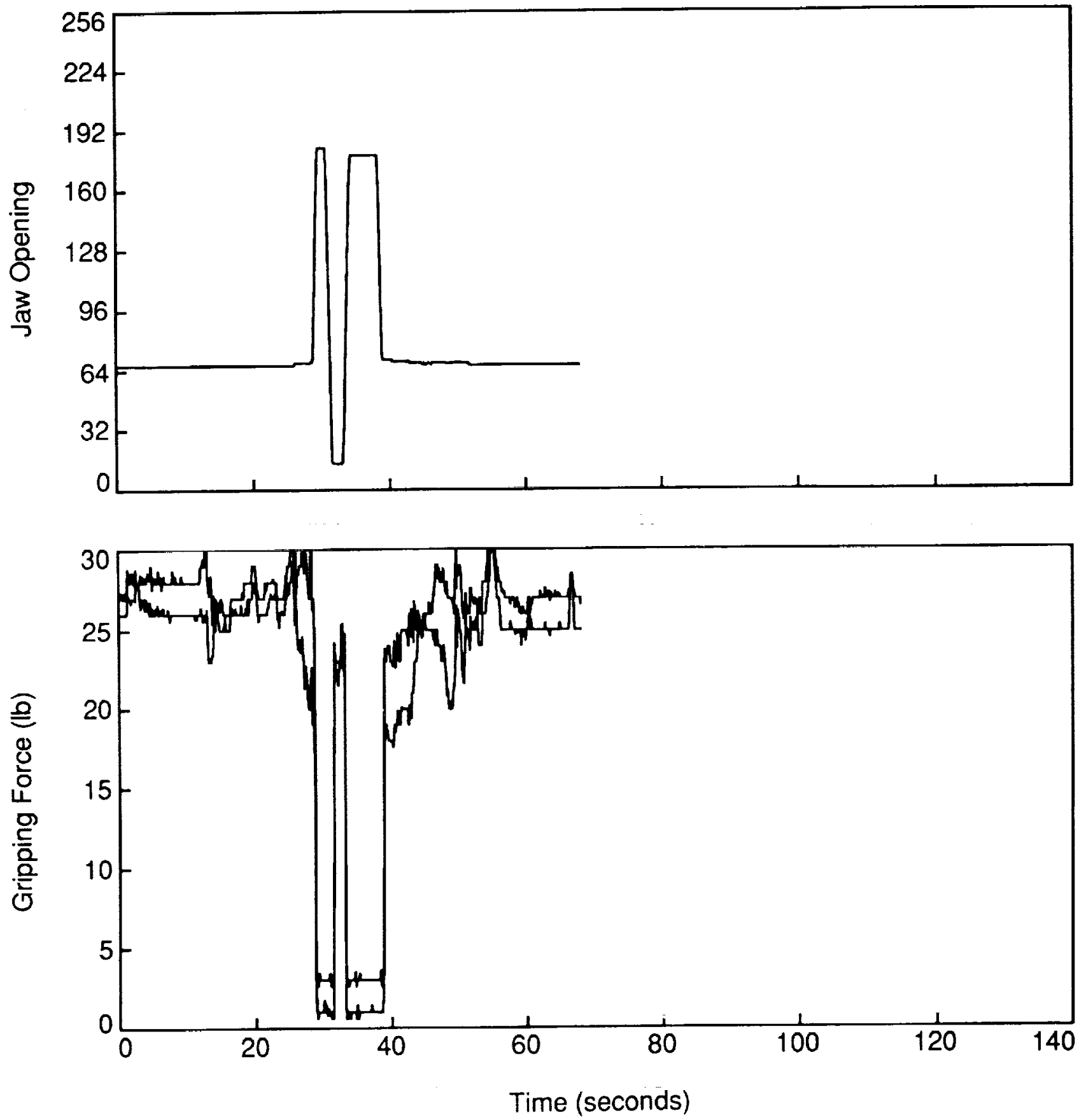

Figure 6. Jaw opening and finger gripping force for the peg-in-hole task. Jaw opening in arbitrary units, gripping force (one trace for each finger) in pounds. Jaws open after insertion ( $t=25$ seconds), close and open for the tap $(t=27-28$ seconds) and close again on the peg prior to extraction ( $t=39$ seconds). 
TABLE 1

DATA RECORDING CHANNELS

\begin{tabular}{llll}
$\#$ & NAME & RANGE & SOURCE \\
\hline 1 & Jaw Position & $0-255$ units & Hand-position transducer \\
2 & Finger Force 1 & $0-100 \mathrm{lb}$ & Left-finger group force sensor \\
3 & Finger Force 2 & $0-100 \mathrm{lb}$ & Right-finger group force sensor \\
4 & $x$ Force & $0-10 \mathrm{lb}$ & 6-axis wrist force-torque sensor \\
5 & $y$ Force & $0-10 \mathrm{lb}$ & 6 -axis wrist force-torque sensor \\
6 & $z$ Force & $0-10 \mathrm{lb}$ & 6 -axis wrist force-torque sensor \\
7 & $x$ Torque & $0-40 \mathrm{in}-\mathrm{lb}$ & 6 -axis wrist force-torque sensor \\
8 & $y$ Torque & $0-40 \mathrm{in}$-lb & 6 -axis wrist force-torque sensor \\
9 & $z$ Torque & $0-40 \mathrm{in}$ - $\mathrm{lb}$ & 6 -axis wrist force-torque sensor
\end{tabular}

\section{Task Board}

The task board (Figure 7 ) consists of a 21-in. by 21-in. frame which accepts modules of either $7 \mathrm{in}$. by $7 \mathrm{in}$. or $14 \mathrm{in.} \mathrm{by} 7 \mathrm{in}$. The frame is adjustable in its vertical angle and can fold down completely for compact storage. The modular design allows for easy construction of new tasks. Modules can be moved or interchanged within the task board. All of our generic tasks have been constructed on the 7 -in. by 7 -in. module size. Detailed information on the task-board design is included in Appendices A and B.

Another advantage of the modular task-board design is that the modules can be mounted individually on a six-axis force-torque sensor to enable force-torque recordings during direct manual operation of the tasks. This would be impractical without the modular design because the large mass of a complete task board would cause enough gravitational force to saturate the sensor. 

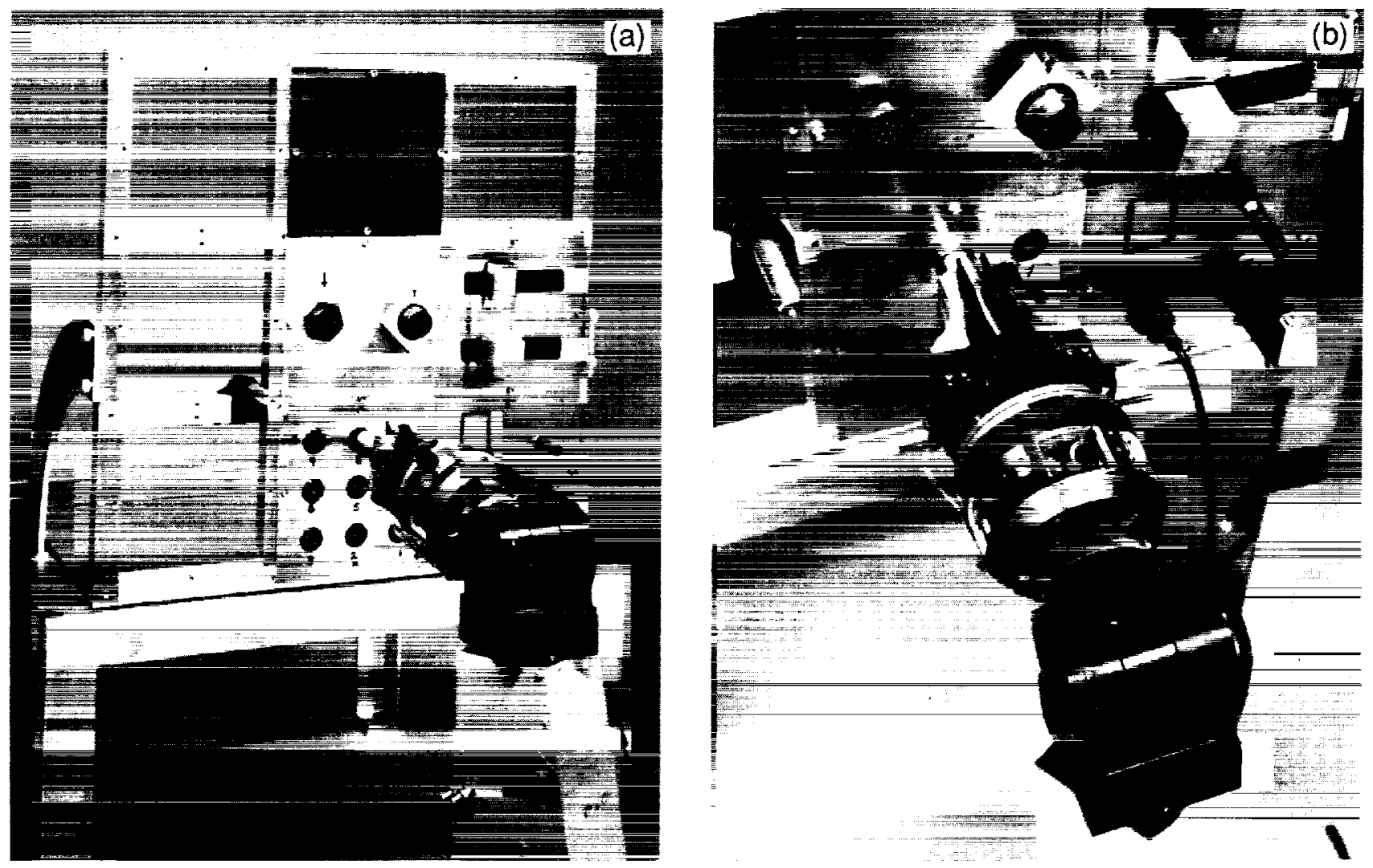

Figure 7. Two views of the task board and the robot hand performing the peg-in-hole task. The modular task board design has nine 7" $\times$ 7" openings for task modules. Shown are the four task modules used in the experiments: (a) Bayonet Connector and Peg-in-hole tasks (upper and lower left modules); (b) Velcro and Electrical Connectors tasks (upper and lower right modules). Task modules could be removed and mounted on a separate force-torque sensor for measurement of force and torques in hand operation.

\author{
ORIGINAL PAGE \\ BLACK AND WHITE PHOTOGRAPH
}




\section{Tasks}

The four tasks used were attach and detach velcro, peg insertion-extraction, manipulating three electrical connectors, and manipulating a bayonet connector. Each task in turn is broken down into component subtasks:

Task 1 Velcro attachment. Exchange the position of two differently shaped blocks attached to the task-board module with velcro. Attempt to attach the blocks securely while minimizing unnecessary force.

Task 2 Peg-in-hole matrix. This task consists of nine holes arranged in a square matrix. The rows each have a progressively larger clearance, and each column has a different chamfer. The subtasks are to take the standard peg and insert it into a given hole. The dimensions of this task were verified after performance of the experiments and are shown in Figure 8.

Task 3 Electrical connectors. The subtasks consist of the mating and unmating of three standard clectrical connectors: a three-prong chassis power-cord connector, a DB25 25-pin signal connector, and a 1/4-in. telephone-style plug.

Task 4 Bayonet connector. This task consists of unlocking, unmating, mating, and locking a Bendix bayonet-style electrical connector.

\section{Task Details}

Peg-in-hole is a popular task because it is easy to specify and thus can be, and has been, duplicated in many labs. In comparing peg-in-hole tolerances among studies in the literature, a useful measure is an information measure of precision, $I$ :

$$
I=\log _{2}(H /(H-P))
$$

where $H$ is the hole diameter and $P$ is the peg diameter. This measure is tabulated in Table 2 for some recent telemanipulation studies which used the peg-in-hole task. These dimensions can be placed in the context of industrial practice using Figure 9.

\section{TABLE 2}

STUDY

Hill \& Salisbury 1977

Hill 1979

McGovern 1974

Pepper \& Kaomea 1988

Present Study
HOLE SIZE

(in.)

2.000

1.000

$2.550-12.540$

0.254

$1.003-1.0006$
PEG SIZE

(in.)

$1.000-1.996$

$0.250-0.984$

2.540

0.295-0.386

0.998
INFORMATION RANGE, I (bits) Figure 9 symbol

$\begin{array}{ll}1.0-9.0 & \triangle \\ 0.4-6.0 & \star \\ 0.3-8.0 & \circ \\ 2.0-5.6 & \diamond \\ 6.0-8.6 & \bullet\end{array}$


BOARD DIMENSIONS
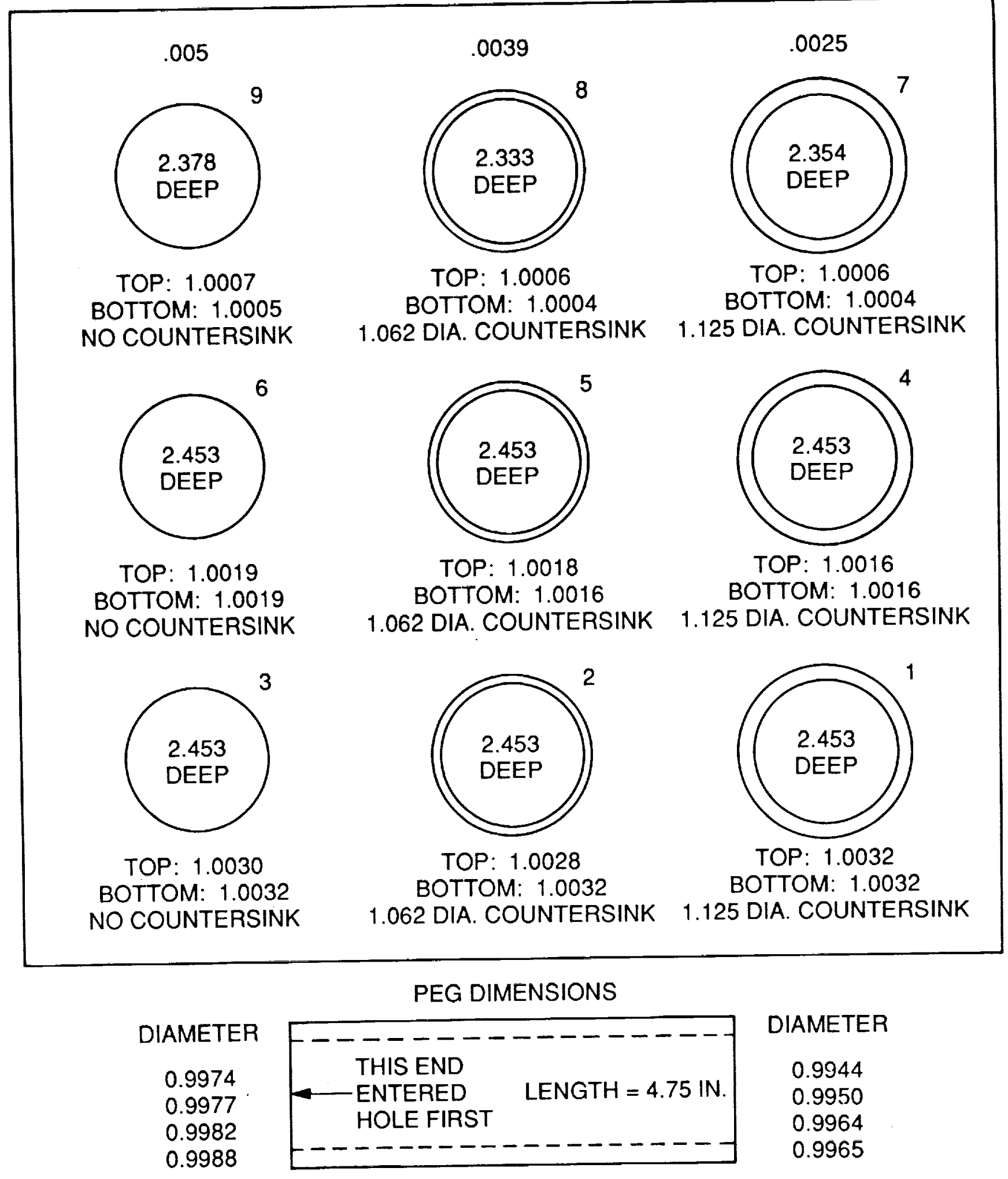

Figure 8. Dimensions of the holes and peg used in the peg-in-hole task. 


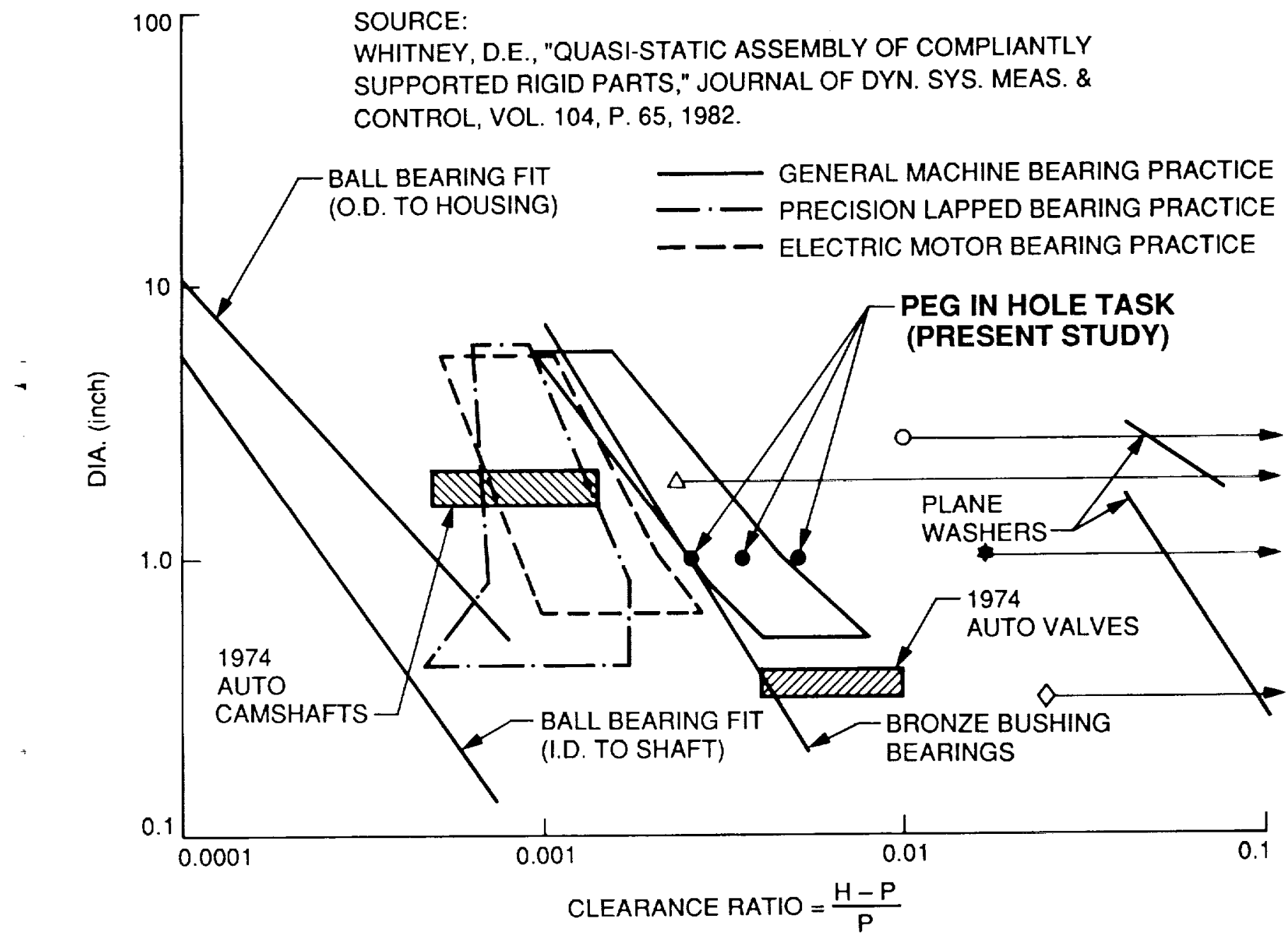

Figure 9. Comparison of tolerances used in the peg-in-hole task with industrial design practice. 
Note that this measure is different from similar "Fitts" measures used by Hill (1972) and Pepper et al. (1988). Our measure ignores the translation component and focuses instead on the difficulty of insertion and extraction of the peg once the tip is properly positioned. The Fitts measure incorporates the translation portion of the peg-in-hole task as:

$$
I_{F}=\frac{1}{T} \log _{2}\left(\frac{2 A}{H-P}\right)
$$

where $A$ is the distance moved to reach the hole and $T$ is the time to complete the movement and peg insertion. Our peg-in-hole task included about 6 in. of translation which was usually accomplished in about $10 \mathrm{sec}$. Thus, the maximum Fitts rate of our task was about $12 \mathrm{bits} / \mathrm{sec}$. This type of analysis is more thoroughly reviewed by Wiker (1988).

The other tasks used here are similarly easy to specify because they use commercially available components. The electrical connectors were unmodified except for a square gripping fixture attached to the locking ring of the Bendix multipin connector (type \#PT06A$20-16 \mathrm{~S} / 16 \mathrm{P})$, and velcro is a commonly available material.

\section{Task Sequences}

Each task was performed according to a prespecified procedure for which the subjects (operators) trained. The task sequences were interspersed with "taps," in which the operator made momentary contact with a designated point on the task board either with the bare gripper or with a held object. The taps injected distinct spikes in the force record (especially the X axis, normal to the task-board surfacc, see Figure 4). All tasks began and ended with a tap on a designated square on the task-board surface. The resulting forcc spikes provided well-defined benchmarks for measurement and interpretation of the progress of the task by inspection of the force records alone.

The tasks, subtasks, and sequences for the tasks are listed in Appendix C.

\section{Test Operators}

The test operators for this experiment were chosen from a population that would have technical background, but not an in-depth knowledge of robotic technology. Detailed robotic knowledge or knowledge of the specific system itself was felt to be distracting and to not reflect forseeable operator populations. Subjects recruited were graduate students, undergraduate students, and one technician, none of whom were specialists in robotics.

\section{Pretesting and Training}

Before telemanipulation training, the subjects performed two standard tests designed to asscss motor-control ability. These tests were administered automatically by a graphics workstation. The motor control battery consisted of a rotary pursuit tracking task and a mirror tracing task. In the first task, the operator controlled a small green dot and attempted to keep contact between the dot and larger red dot moving in a circle at approximately 33 revolutions per minute. In the second, the operator drew the outline of a star between two path boundary lines in the presence of $0-, 90-, 180$, and 270-degree rotations 
of cursor motion relative to hand motion. The tracking-task performance was measured by percentage of the time on target; the star-tracing task by percentage of line length within the displayed outlines. The subjects used a mouse to interact with the computer; the tasks were completely controlled and scored by the computer.

After the "motor control" testing, each subject received 2 to 4 hours of practice on the telemanipulation apparatus. The practice sessions consisted of four 30 -minute sessions in which the task set was performed with and without force feedback.

\section{ANALYSIS AND PERFORMANCE MEASURES}

The raw force-torque data are a rich source of information which can be understood in terms of the task description and which can in turn be used to quantify task performance. This section will describe computations which were performed on the force/torque data to produce performance measures.

Data recorded at such a high rate $(100 \mathrm{~Hz})$ were computationally difficult to analyze and contained spurious information such as mechanical ringing signals showing up in the force/torque plots (Figure 10). To simplify plotting and analysis, the data were filtered and reduced by a decimation process which averaged groups of 10 successive samples to produce a filtered output data file. Figure 11 shows the resulting data in which mechanical oscillations above $5 \mathrm{~Hz}$ are suppressed.

\section{Completion Time}

The simplest and most long-standing task performance measure, completion time, can be determined from the length of the data file containing the force-torque data. The length of the data file was determined by the time between the experimenter's button presses. In most of the cases reported here the movement time was long compared to human reaction time and thus errors due to reaction-time variability of the experimenter are minimal. To prevent even this small error, our analysis software identified the starting and ending taps and used them as the basis of completion time.

\section{Sum of Squared Force (SOSF)}

This measure is computed by taking a nondecreasing sum of the square of the force or torque value

$$
\operatorname{SOSF}=\sum_{i}^{N} f_{i}^{2} d t
$$

where $N=$ number of data samples (task time over $d t), f_{i}$ is the $i$ th sample of force or torque, and $d t$ is the sampling interval ( $0.01 \mathrm{sec}$ in this experiment). SOSF is accumulated separately for each force and torque axis. An alternative and related force/torque performance measure is RMS force/torque. RMS force/torque is not preferred because it cannot be added among different movements, channels, or segments. Adding the RMS measure biases the resulting sum in favor of shorter segments or tasks. As with completion time, SOSF can be computed over the whole record or between starting and ending taps. 

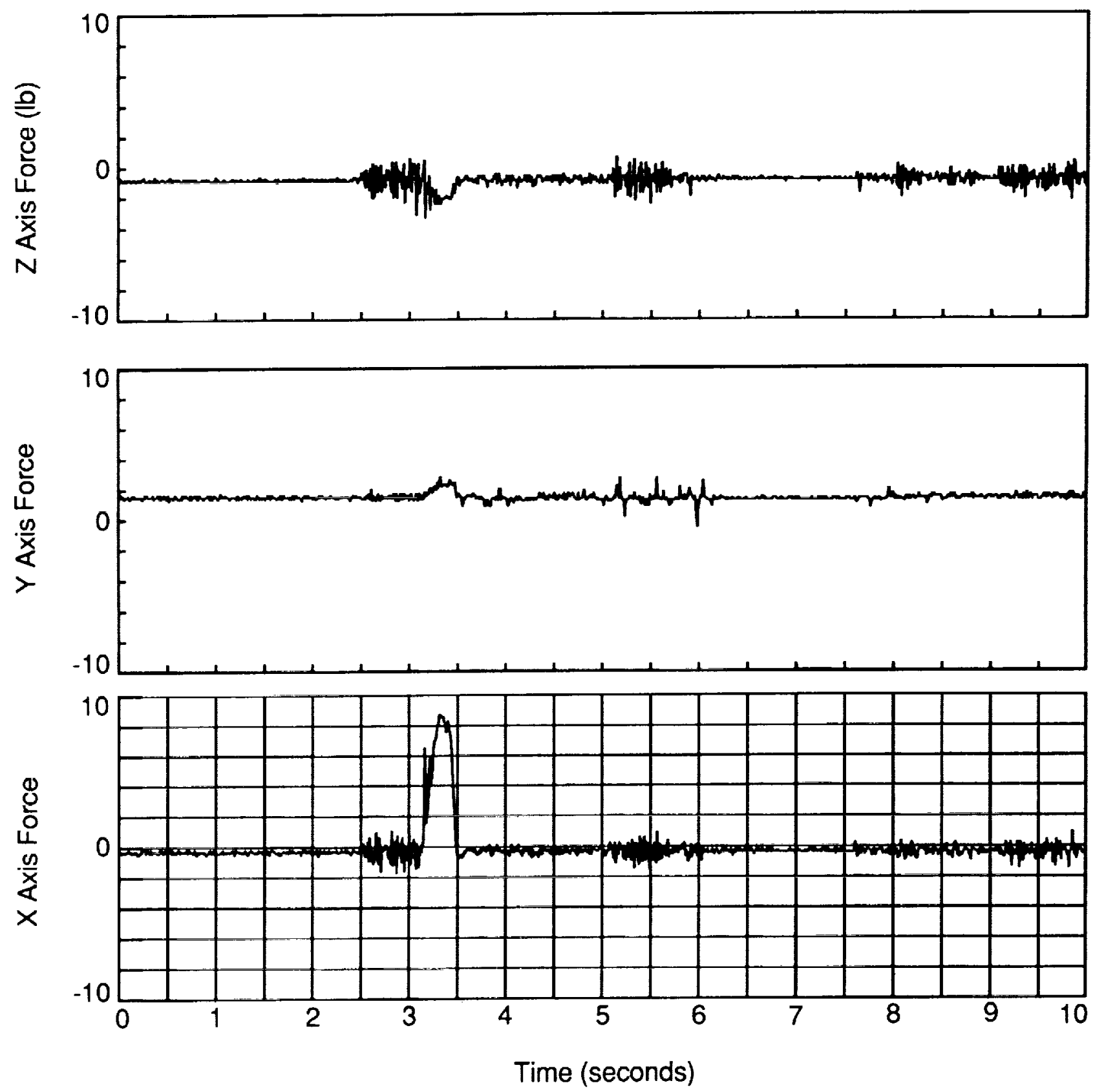

Figure 10. Unprocessed data from the force-torque sensor. Note expanded time scale. Data was filtered by averaging adjacent non-overlapping windows of 10 samples (see Figure 11). 

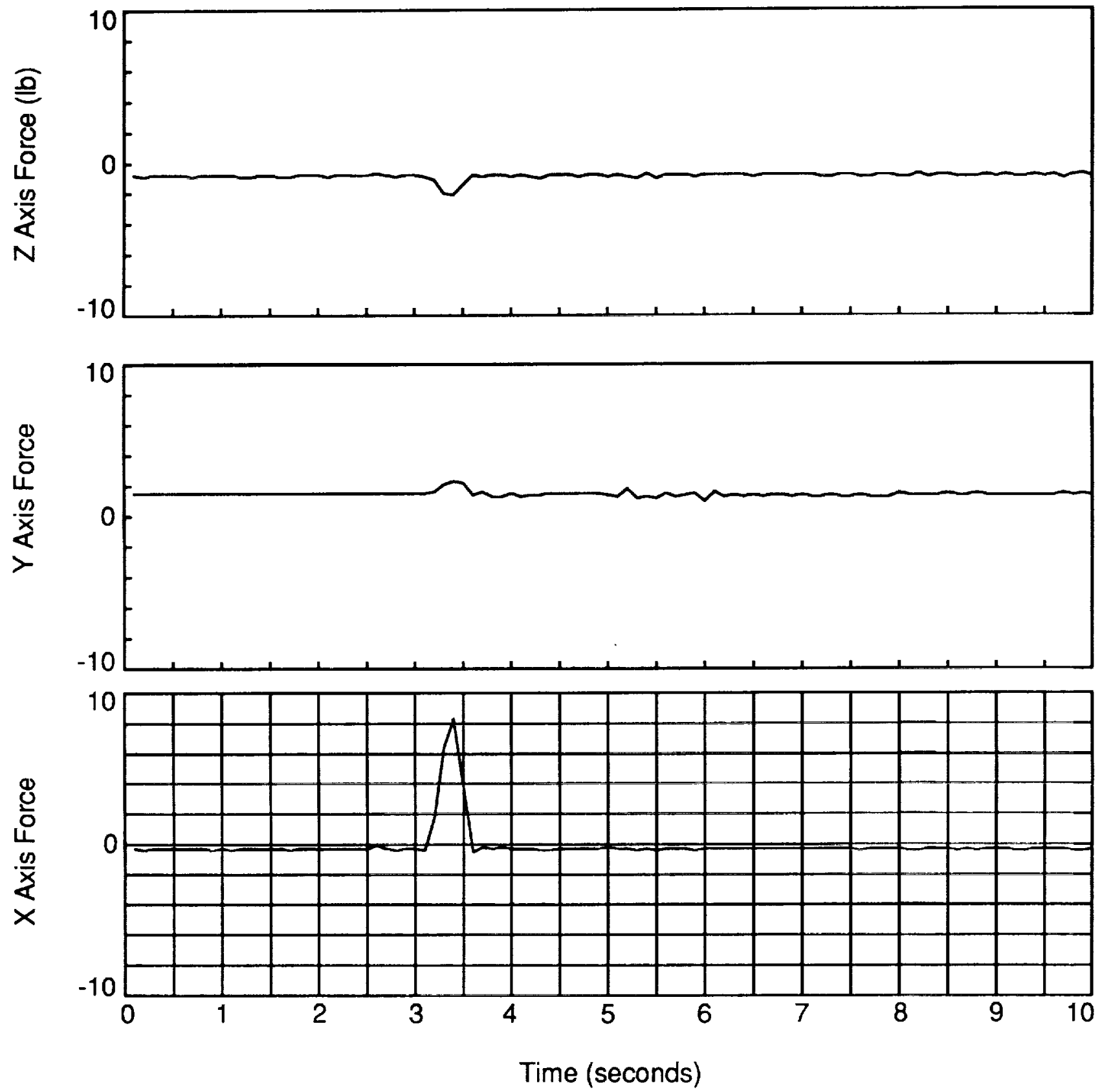

Figure 11. Filtered data. Same data as in Figure 10, but filtered with 10-sample averaging window. 


\section{Visual Error Scoring}

A third way in which performance can be measured is through an observer's notations of the "quality" with which a task is performed. In our experiments, a set of "errors" was defined and explained to the test operators and experimenters (see Table 3). An experimenter watched each repetition of the experiment and counted occurrences of each error.

\section{TABLE 3}

\section{Task Errors}

SYMBOL NAME

\begin{tabular}{ll}
\hline S & Slip \\
I & Incomplete \\
GI & Gripper Interference \\
D & Drop \\
E & Excessive Force
\end{tabular}

\section{DEFINITION}

\section{Object slips in jaws.}

An inserted object such as a peg is not completely inserted.

Gripper touches an object not related to the current task.

Gripped object is dropped.

Visible deflection of the task board.

Individual differences among error scorers might be a source of bias in this measure. To detect this possible bias, four of the experimenters scored the same experimental run. Their error counts were substantially in agrcement.

\section{Task Segmentation Analysis}

In some cases it is desirable to compare performance measures among different segments of the same task. For example, to compare the completion time and SOSF for peg insertion with peg extraction. This was accomplished through a computer program which could recognize benchmarks in the force signal, and divide it in time between a set of segments. Returning to the peg-in-hole example (Figure 12a, b, c, sce especially " $x$ force" in part a), the data can be clearly divided into "translation" (the manipulator is in free motion; no contact forces), "taps" (sharp spikes in force), "insertion" (predominantly positive forces), and "extraction" (predominantly negative forces). Both "completion time" and SOSF can thus be computed for each segment of the task. This could potentially identify which task segments are more or less affected by a particular system improvement.

The segmented analysis program uses a description of the expected task sequence entered by the operator. This description is entered in a simple language expressing a rough model of the expected forces developed during performance of a task. The elements of this language are single letters describing typical force patterns (see Table 4). Referring to the task description of the peg-in-hole task for example, the experimenter can write the expected force sequence as:

$$
\text { " } \mathrm{m}, \mathrm{t}, \mathrm{m}, \mathrm{i}, \mathrm{m}, \mathrm{t}, \mathrm{m}, \mathrm{x}, \mathrm{m}, \mathrm{t}, \mathrm{m} "
$$




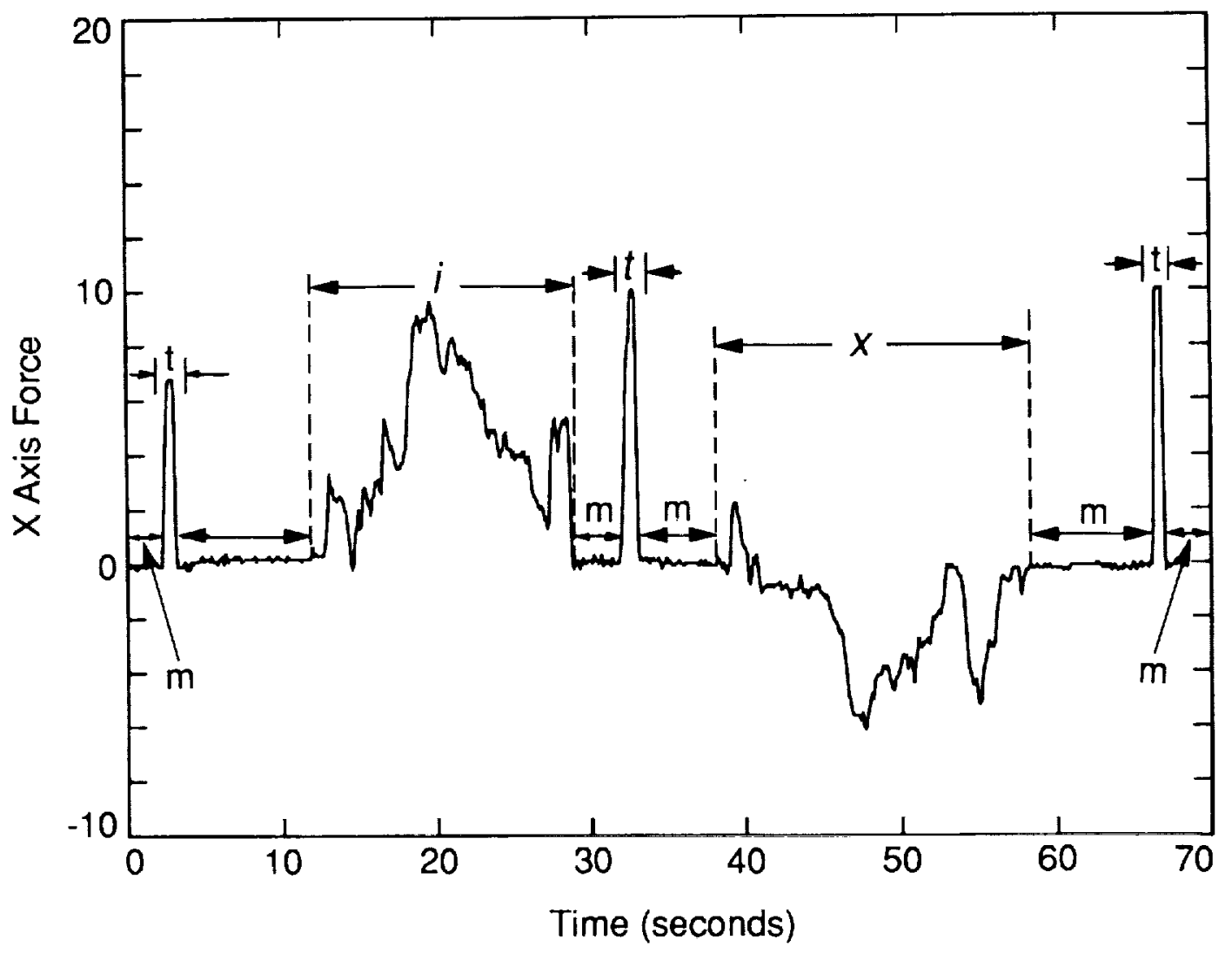

Figure 12. Segmentation of task performance data: shown is an example of $x$ axis force data from the peg-in-hole task automatically divided into phases by the segmentation algorithm. Designation of phases is:

$m$ Move: translation phases of the task involving no contact forces.

$t$ Tap: momentary intentional impact with task board surface - a timing mark.

$i \quad$ Insert: forces arising from insertion of the peg.

$x \quad$ Extract: forces arising from extraction of the peg. 


\section{Table 4}

\section{Task Phase Description Language}

\section{Symbol Definition}

\begin{tabular}{ll}
\hline $\mathrm{m}$ & Movement: free motion of the slave, contact forces are negligible. \\
$\mathrm{t}$ & Tap: a sharp force impulse. \\
$\mathrm{i}$ & Insertion: sustained predominantly positive force. \\
$\mathrm{x}$ & Extraction: sustained predominantly negative force.
\end{tabular}

\section{Segmentation Algorithm}

The segmented analysis program uses this description to drive a thresholding state machine which identifies the time at each transition. This is accomplished by first identifying the taps in the data.

Each tap separates the task into smaller actions. Before and after each tap is a short period of time when no forces are exerted on the hand. This enables us to separate the taps from the rest of the data. The algorithm finds the taps in the $x$-force data so that completion time and SOSF analysis can be done on the individual actions. A tap is defined by several different parameters including minimum height, $h$, maximum width, $w$, minimum time before the tap when no forces occur, $t b$, minimum time after the tap when no forces occur, te, tolerance to allow for noise during the periods before and after the tap, tol, and finally the maximum time between the beginning of the tap (when the force crosses the tolerance level) and when the force is greater than or equal to the minimum height, $t_{21}$ (Figure 13).

The tap algorithm is split up into four states. In each of these four states, specific conditions must be met to cause a transition to the next state; if not, the algorithm jumps back to state 1 and analysis continues until the end of the data.

State 1 Move forward through the $x$-force data until the end of the data or until crossing the minimum height (force) threshold, $h$. Mark this position.

State 2 Jump backward through the $x$-force data the minimum length of time for a transition before the tap, $t b$. Step forward in the $x$-force data until the force exceeds the tolerance tol. Mark this position. Check to see if the length in time between this mark and the mark from state 1 is within the limit defined by the tap definition, $t_{21}$. If not, go back to state 1 ; if so, proceed to state 3 .

State 3 Jump forward in the $x$-force data from the state 1 mark to the width of a tap, $w$, plus the minimum length of time for a transition, $t e$. Step backwards until the force jumps outside of the tolerance, tol, and mark the position. Check to see if the length in time between this point and the state 2 marker (the beginning of the tap) are within the definition of a tap width, $w$. If not, go back to state 1 ; if so, proceed to state 4 . 


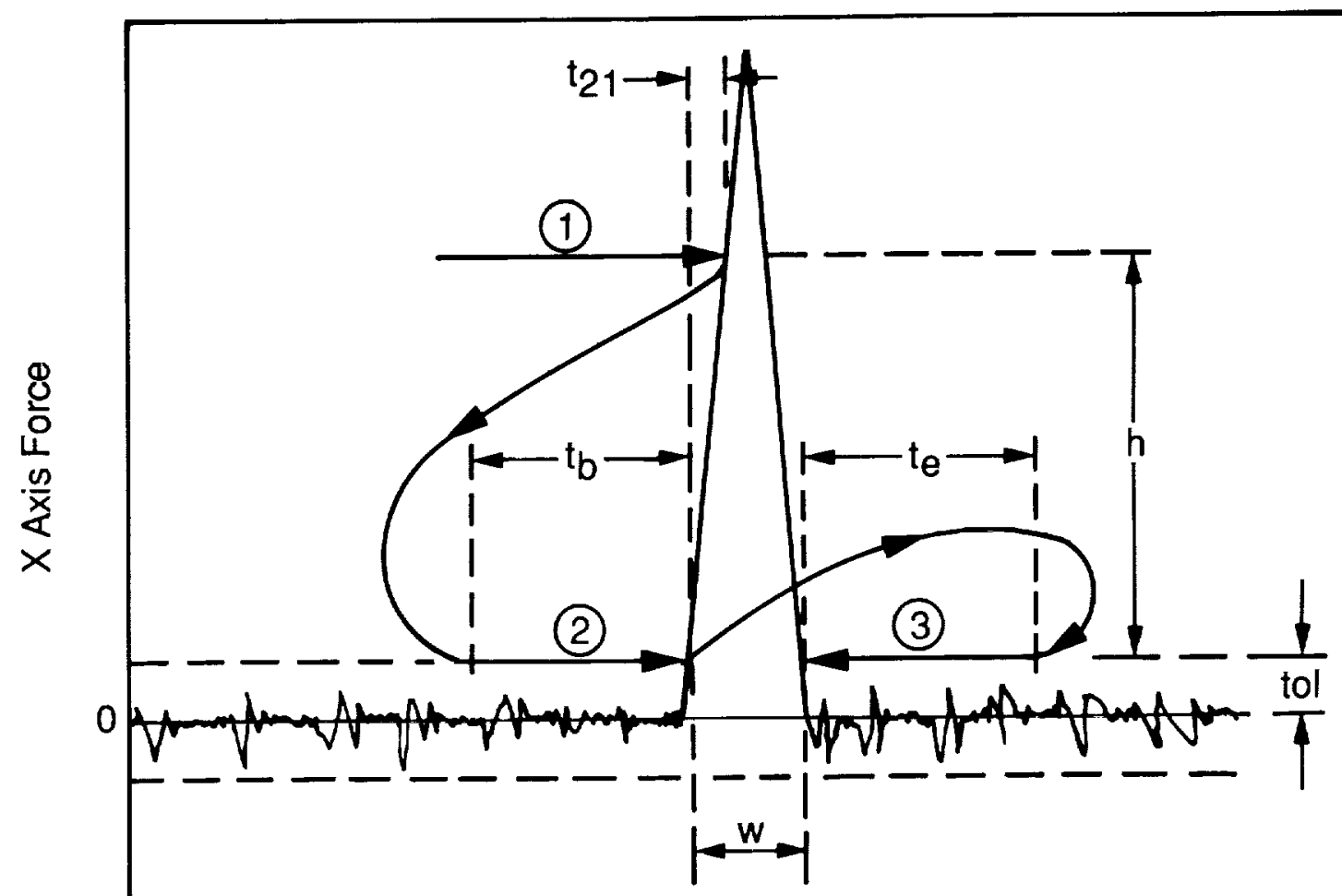

Time

$\mathrm{h}=$ Minimum Tap Force

$\mathrm{w}=$ Maximum Tap Width (time)

$t_{b}=$ Minimum Contact-free time before tap

$\mathrm{t}_{\mathrm{e}}=$ Minimum Contact-free time before tap

tol $=$ Noise Threshold

$t_{21}=$ Front Porch Time

(1) State 1
(2) State 2
(3) State 3

Figure 13. Diagram of algorithm for identification of a "tap," a voluntary impact designed into the task definition to act as an event marker. The algorithm is a state machine driven by threshold crossing events. The six parameters define the permissible variation in tap height, width, etc. 
State $4 \mathrm{~A}$ tap has been found. Store the beginning and end of the tap and calculate the center of the tap. Return to state 1.

After the taps are located, the movement time is calculated, and boundaries are defined for the insertion and extraction phases. Completion time and SOSF can then be accumulated for each task phase. 


\section{RESULTS}

\section{SUMMARY RESULTS}

The results of the experiments form a complex set of data points on the three-way experimental design. Each point in the three-dimensional space formed by the control modes, subjects, and tasks contains three reduced-data points for each of the three repetitions of each experimental trial for each of the five subjects. These are the completion time, SOSF, and number of errors.

The reduced data can be further simplified by averaging across one or more dimensions of the design. As a first look at the data, we have computed averages over all subjects and over the first three tasks, "velcro," "peg-in-hole," and "electrical connectors." The fourth task was not included in this average because it took significantly longer than the others (approximately 150 seconds vs. 75 seconds), and was often not completed due to its difficulty. There are nine subtasks for the peg-in-hole task (corresponding to the nine test holes) vs. two for the velcro and three for the electrical connectors. These averages (Figures 14,15 , and 16) show clear trends in performance as the level of capability progresses from position control, through force reflection, up to the bare-handed operator.

Completion time (Figure 14) for the three primary tasks drops from an average of 92 seconds with position control to an average of 63 seconds when force feedback is added. Completion time drops to only 14 seconds for the bare-handed human. SOSF (Figure 15) drops even more dramatically (from about 3500 to $500 \mathrm{lb}^{2}$-sec) when pure position control is augmented with force feedback and further (to $200 \mathrm{lb}^{2}$-sec) for the bare-handed case.

The number of errors observed per repetition drops from 3.0 to 1.1 when force feedback is added. No errors were observed in the bare-handed data.

The difference between the first two cases and the bare-handed case is so great as to not require statistical validation. The two-tailed $\mathrm{Z}$ test was used to test the null hypothesis that the differences between position control and force-feedback control performance were due to chance. The number of subtask repetitions performed under each control mode is shown in Figure 17. Because the number of data points is large compared to one (approximately 130 to 150 ), the $\mathrm{Z}$ statistic could be used.

The probability of the null hypothesis calculated by this procedure was much less than 0.01 in all of the differences reported above, giving them a high degree of statistical significance.

These results summarize one of the main results of this report, that the provision of force feedback reduces completion time for a task mix emphasizing energetic interaction and precision manipulation by approximately 30 percent, reduces SOSF by a factor of 7 , and reduces errors in performing the task by 63 percent. 


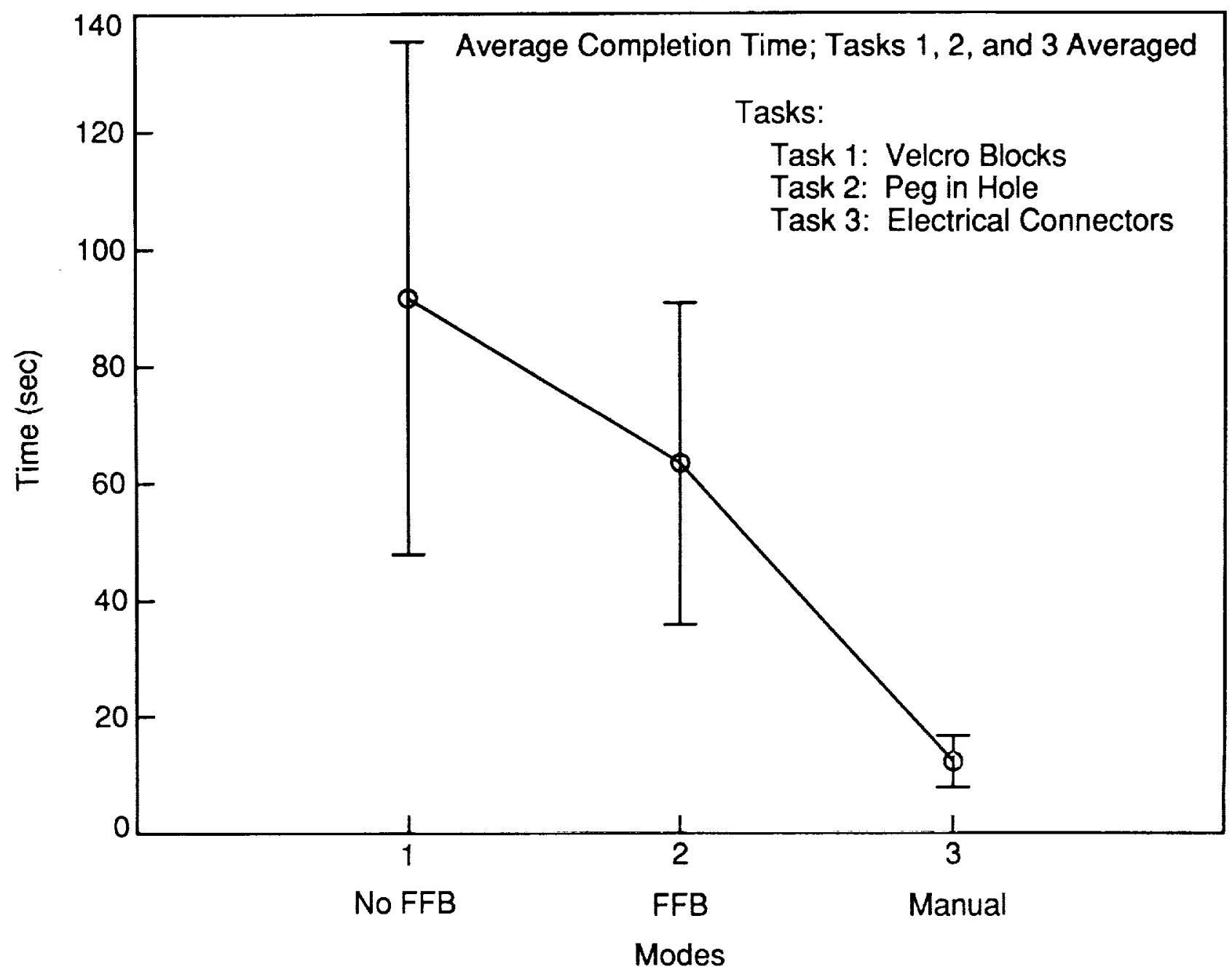

Figure 14. Average completion time performance for five operators performing a mix of tasks under three conditions: (1) position control only, (2) position control with kinesthetic force feedback, (3) direct operation with the bare human hand. 


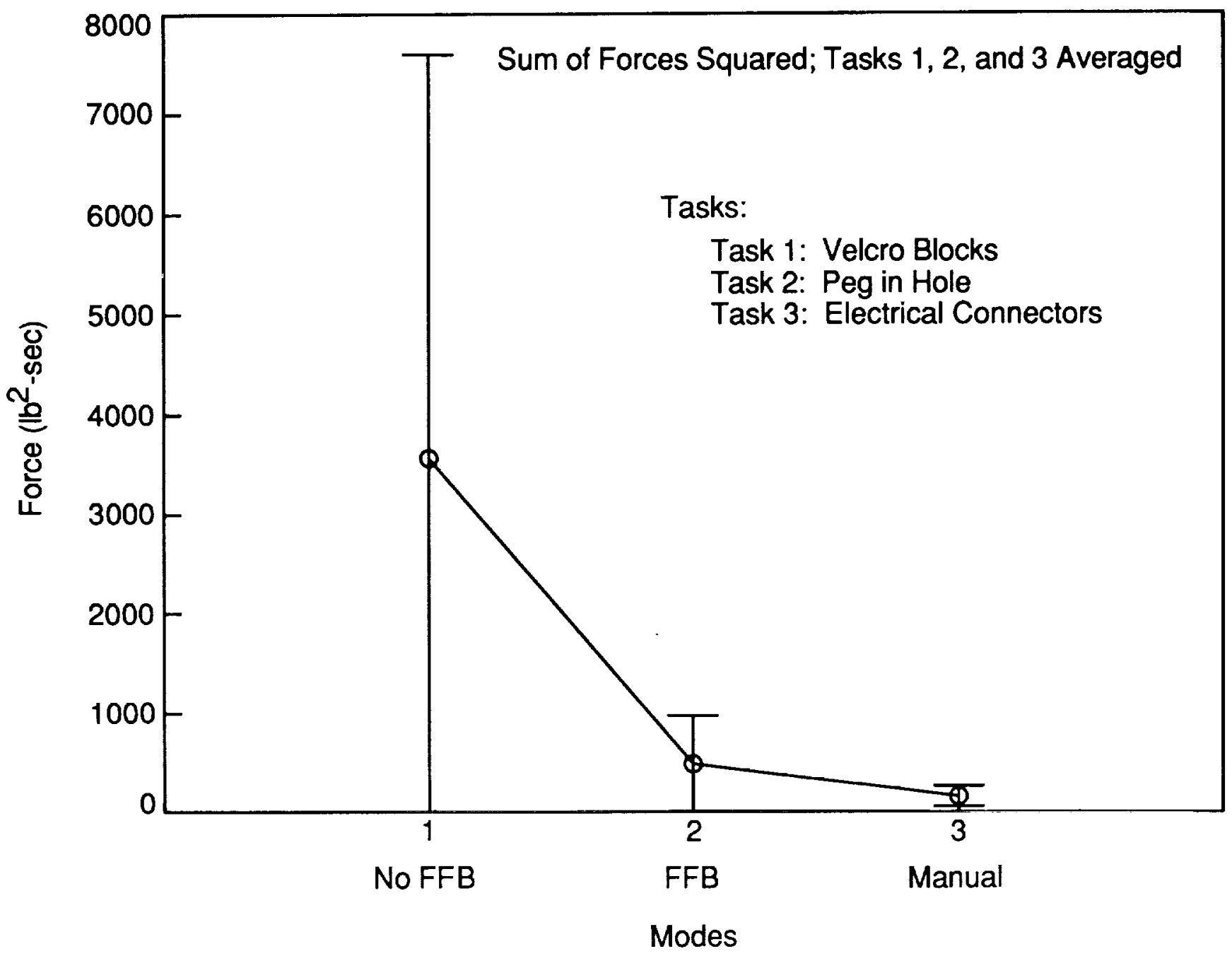

Figure 15. Sum of Squared Forces (SOSF) performance measure for the same operators and tasks as in the previous figure. SOSF is a measure of the total magnitude of force required to perform the task. SOSF drops dramatically as manipulation performance is improved. 


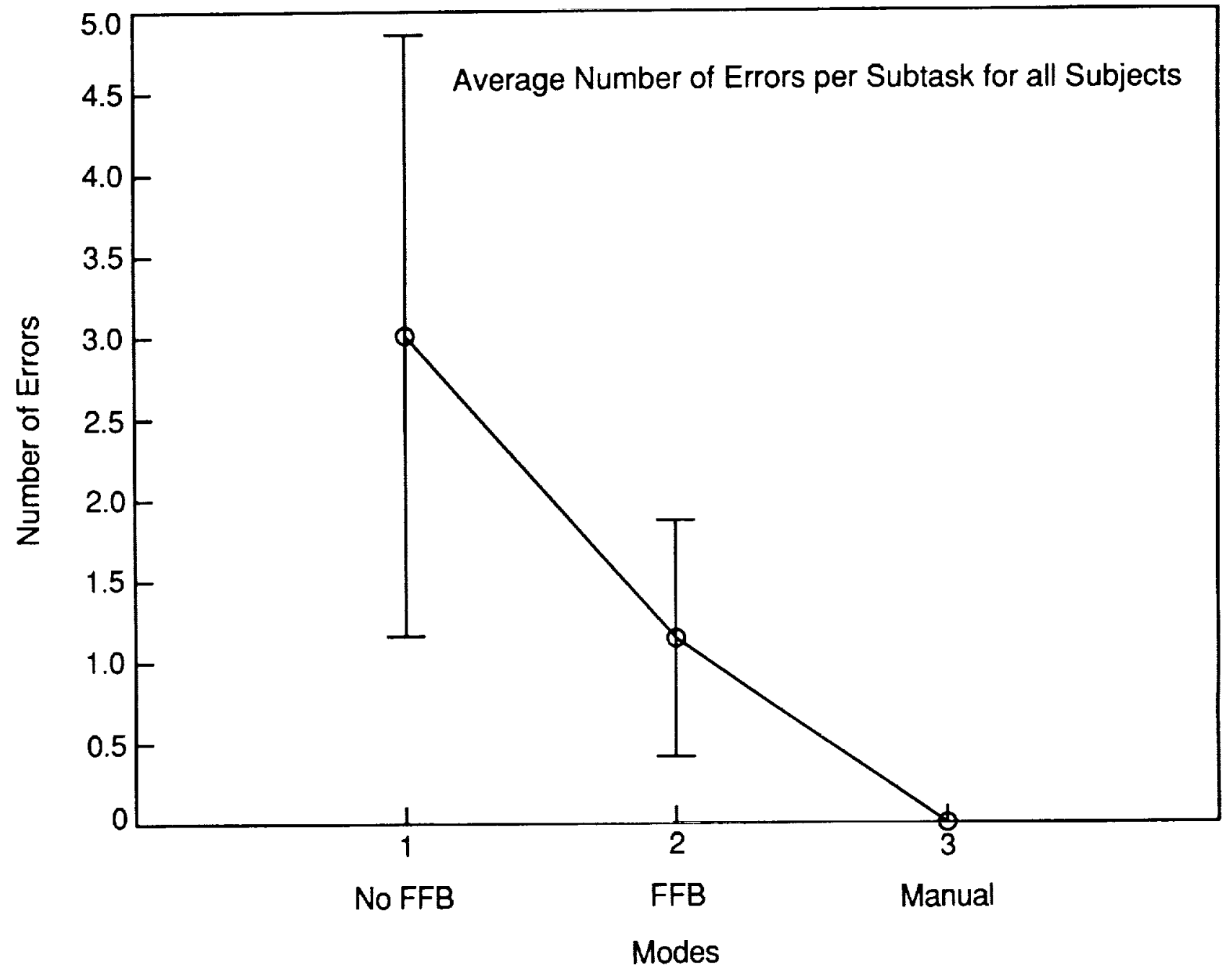

Figure 16. Average number of errors made by the operator in each repetition of each task. The errors were predefined and understood by the test operators. 


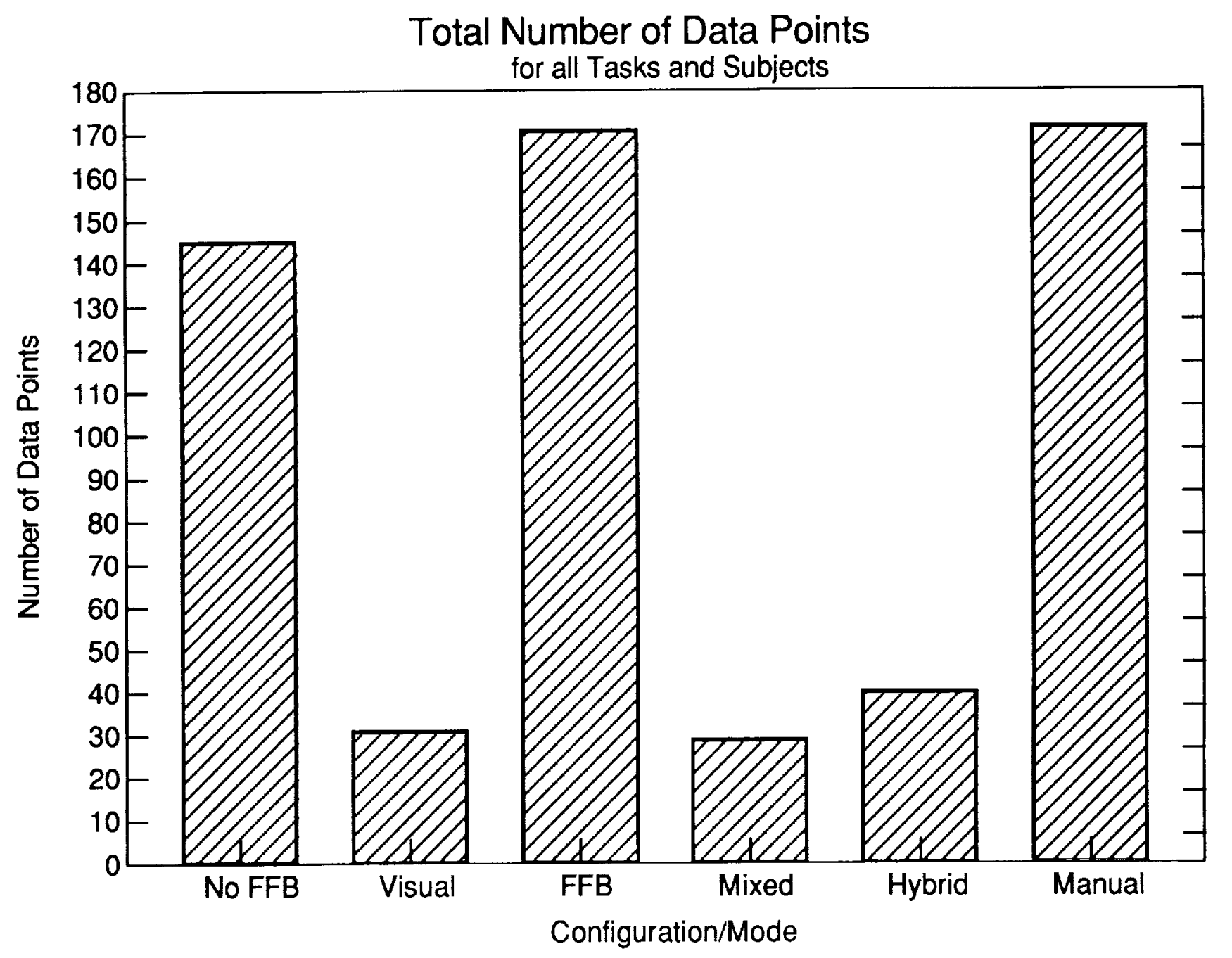

Figure 17. Number of data points for all of the experiments. Each data point represents one subject doing a single performance of one of the subtasks. Visual, mixed, and hybrid modes were studied as preliminary experiments with fewer repetitions and subjects. 
The motor control pretesting was performed in order to determine whether the performance of individual operators in teleoperation could be predicted from their performance on the pretests. A summary of these results is presented below. For further details, see Appendix D.

The analysis attempted to compare performance on the various motor control tests with performance in the various telemanipulation tasks. Linear regression was computed on the points formed by plotting the performance of each operator on selected axes consisting of one pretest performance measure and one measure of telemanipulation task performance. The approach was to check for statistically significant correlations which would indicate the predictive power of the selected tests. The significance of a particular correlation is determined by the permissible $P$ value (in this case 0.05 ) and the computed value of the corrclation coefficient $r$. For a given number of data points (subjects) there is a threshold on $r$ below which the correlation is not significant. The results were that the number of significant correlations was approximately equal to that expected by chance. Thus the selected motor control tests appear not to predict telemanipulation performance.

Because of the small number of subjects, only very strong correlations can be detected with high confidence. Repetition of similar experiments with more subjects is underway to increase the ability to detect smaller but still significant correlations.

\section{DETAILED RESULTS}

As an example of the raw data resulting from these experiments, the complete, filtered data set for a single repetition of the peg-in-hole task shows a complex coupled set of time functions (Figures $4,5,7$ ) in which forces and torques vary (Figures 4,5 ) as the jaws release and then regrasp the peg (Figure $6, t=25$ to 40 seconds). Recall that the task is defined to start and finish with the peg held in the gripper. The $x$-axis force (Figure 4 , bottom trace) in conjunction with the jaw opening (Figure 6, top trace) provides a clear picture of the task sequence. The operator starts by tapping the grasped peg against the task board and moves to the hole. A large positive $x$-force indicates the struggle to insert the peg, which is complete when the jaws release it at $t=25 \mathrm{sec}$. The jaws reclose on empty space to tap the back of the inserted peg $(t=32 \mathrm{sec})$, and the peg is regrasped at $t=39 \mathrm{sec}$ at which point withdrawal almost immediately begins. After the peg is completely removed and contact forces cease $(t=60 \mathrm{sec})$, there is a short translation to the mark, and a final tap. Similar sample records from the other tasks are reproduced in Appendix E.

When selected experimental records from the several control modes are compared (Figure 18), the $x$-axis force traces tell most of the story for the peg-in-hole task because of the alignment between the task axis and the force/torque sensor's $x$-axis. Comparison of performance in the several control modes shows the reduced completion times and force levels achieved as more capability is added to the system.

In the visual feedback condition (Figure 18) the operator uses position control without kinesthetic force feedback, but receives contact force/torque information by means of the graphic display. With only the visual force/torque information, the task is completed in 

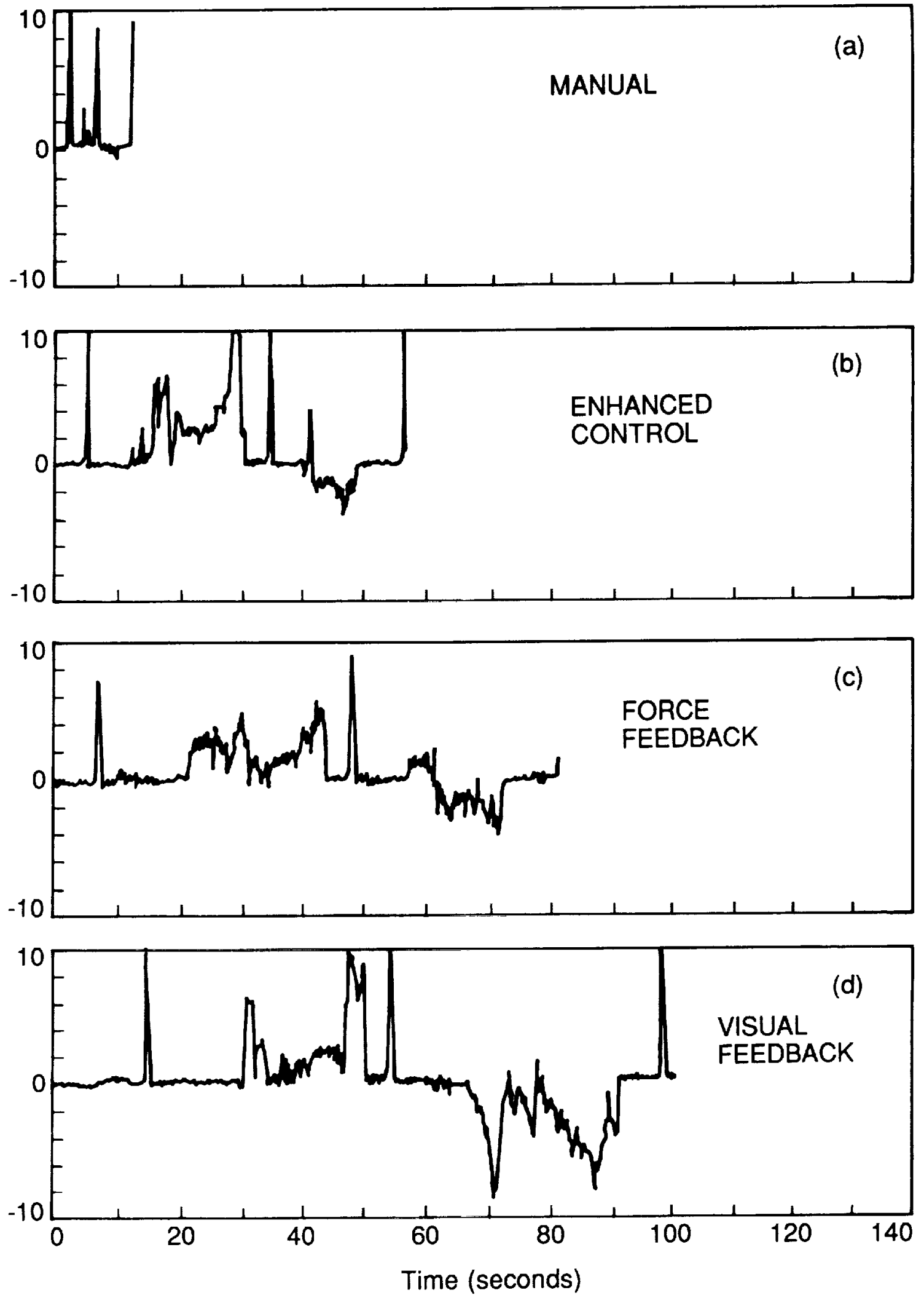

Figure 18. Illustration of force record changes with control modes. Individual records of $\mathrm{X}$ axis force for 4 repetitions of the peg-in-hole task under different control modes. (a) manual, (b) enhanced control; "Hybrid Force Accommodation", (c) force feedback, (d) visual feedback. 
about 100 seconds, and peak forces of up to $+10 \mathrm{lb}$ and $-9.5 \mathrm{lb}$ are observed in the insertion and extraction phases respectively. When kinesthetic force feedback is used (Figure 18b), completion time drops to 82 seconds, and peak forces drop to approximately $\pm 5 \mathrm{lb}$.

Sensed force and torque information can be used locally by the manipulator control system to implement an effective compliance or, in general, a mechanical impedance of the manipulator. Performance with one example of this type of shared control is shown in Figure 18(c). In this mode, the orientation axes wcre controlled automatically to move in the direction of sensed torques. Position axes were controlled by the operator. Completion time was reduced to 60 seconds. Forces were substantially reduced only for the extraction phase, in which the peak force was $-4 \mathrm{lb}$.

Finally, when the task was performed manually (Figure 18d), the task-related forces wcre practically invisible compared to the taps. Completion time was about 15 seconds, and peak forces reached $+3 \mathrm{lb}$ and $-0.5 \mathrm{lbs}$.

Although fascinating in themselves, these raw data records are isolated anecdotes of individual task performances. As described in the summary above, data from all repetitions of the expcriment were reduced to the three basic performance measures. The visually scored error rates were manually collected with the reduced performance data.

The first level of detail added to the summary results was to break them down by individual task instead of averaging all the tasks together (Figures 18a, b, c). Doing this showed that the effect of force feedback was not the same for all of the tasks. For the peg-in-hole task, completion time followed the expected course, dropping by almost a factor of two from 105 to 59 seconds as force reflection was added. For the velcroblocks task, completion time increased from 72 to 83 seconds. Both of these changes were statistically significant when analyzed by the method described above. For the electrical connectors, when force feedback was added only a slight change from the completion time of 70 seconds was observed which was NOT statistically significant. Of course, all of the tasks were completed much faster by the bare-handed operator. The average time in this case was about 15 seconds.

The SOSF data tell a different story. As with completion time, for the peg-in-hole task there was a dramatic drop in SOSF (from 5400 to $500 \mathrm{lb}^{2}$-sec) as force reflection was added. The increase in completion time seen for the velcro task was accompanied by a significant decrease in SOSF (from 800 to $400 \mathrm{db}^{2}$-sec). For the electrical connectors, the SOSF measure declined significantly despite the unchanged completion time.

The data of Figure 19 showed the variation of performance with control mode as task was varied. The same data could be replotted to highlight the task dependence of the effects of mode (Figures 21,22). The first task (positioning velcro blocks) showed no significant effect of force feedback on completion time (see above). The SOSF (Figure 22) was reduced by about $1 / 2$ to a value close to the manual level. The second task, peg-inhole, showed a significant reduction in both completion time and SOSF with the addition of force feedback. 


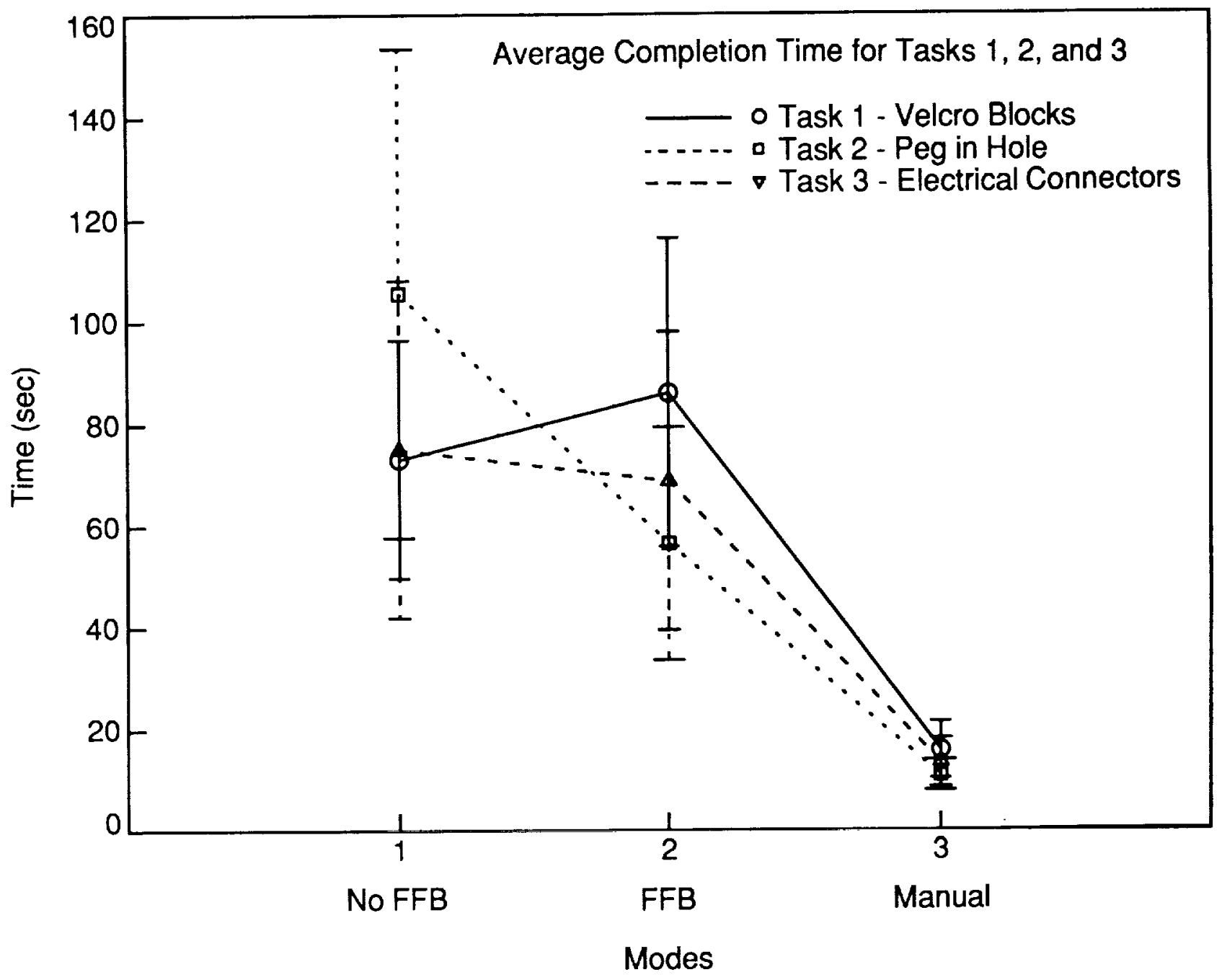

Figure 19. Completion time (CT) as a function of control mode broken down by the individual task type: velcro blocks (solid line, circles), peg-in-hole (dotted line, squares) and electrical connectors (dashed line, triangles). Force feedback affects each task differently. The changes are all statistically significant except the slight drop in CT for electrical connectors with force feedback. Note that force feedback significantly increased $\mathrm{CT}$ for the velcro task. 


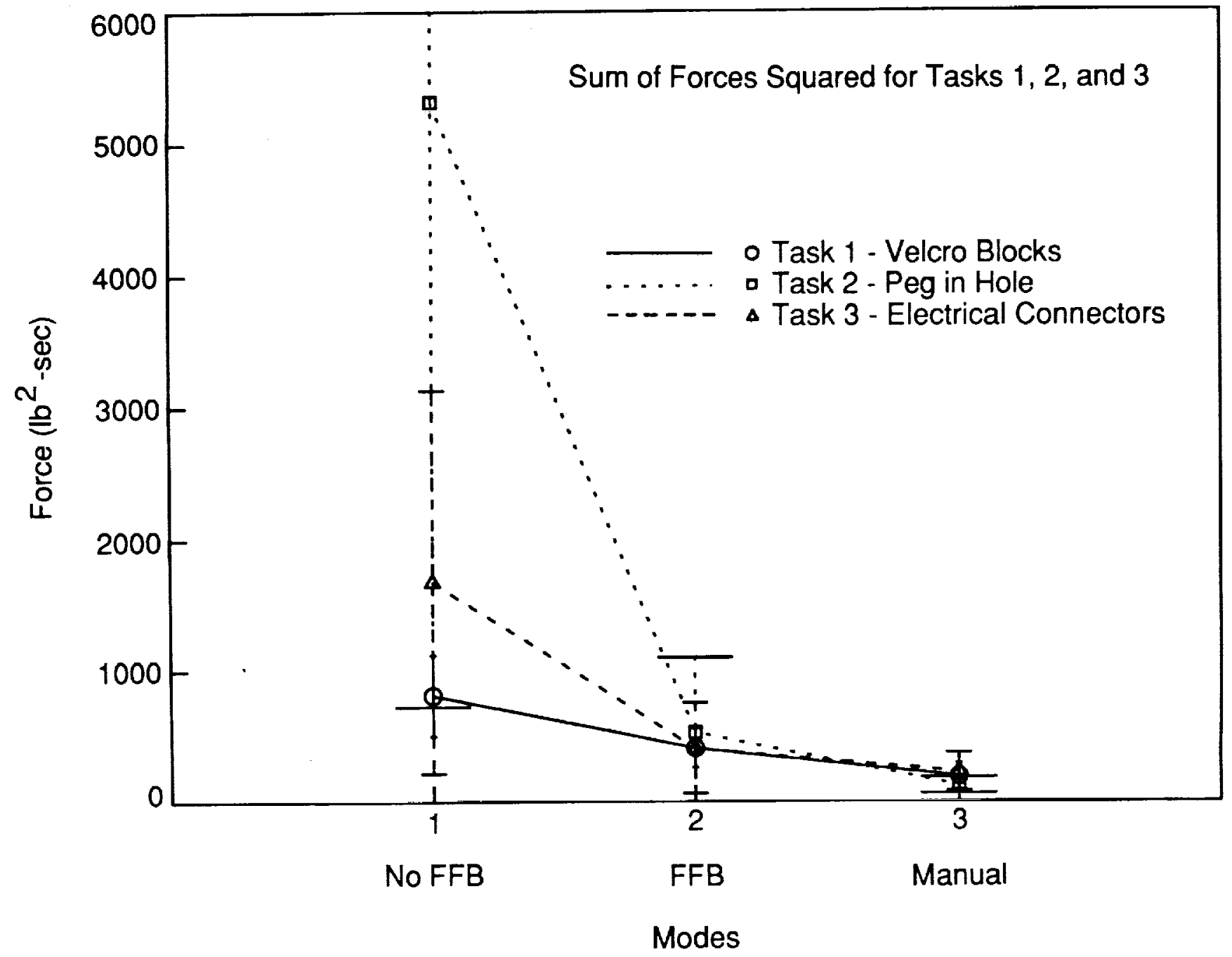

Figure 20. Sum of squared forces (SOSF) measure for the three task types. Key same as for Figure 19. SOSF dropped significantly for all of the tasks when force feedback was used. Levels with force feedback were still approximately double those recorded from the bare-handed operator. 


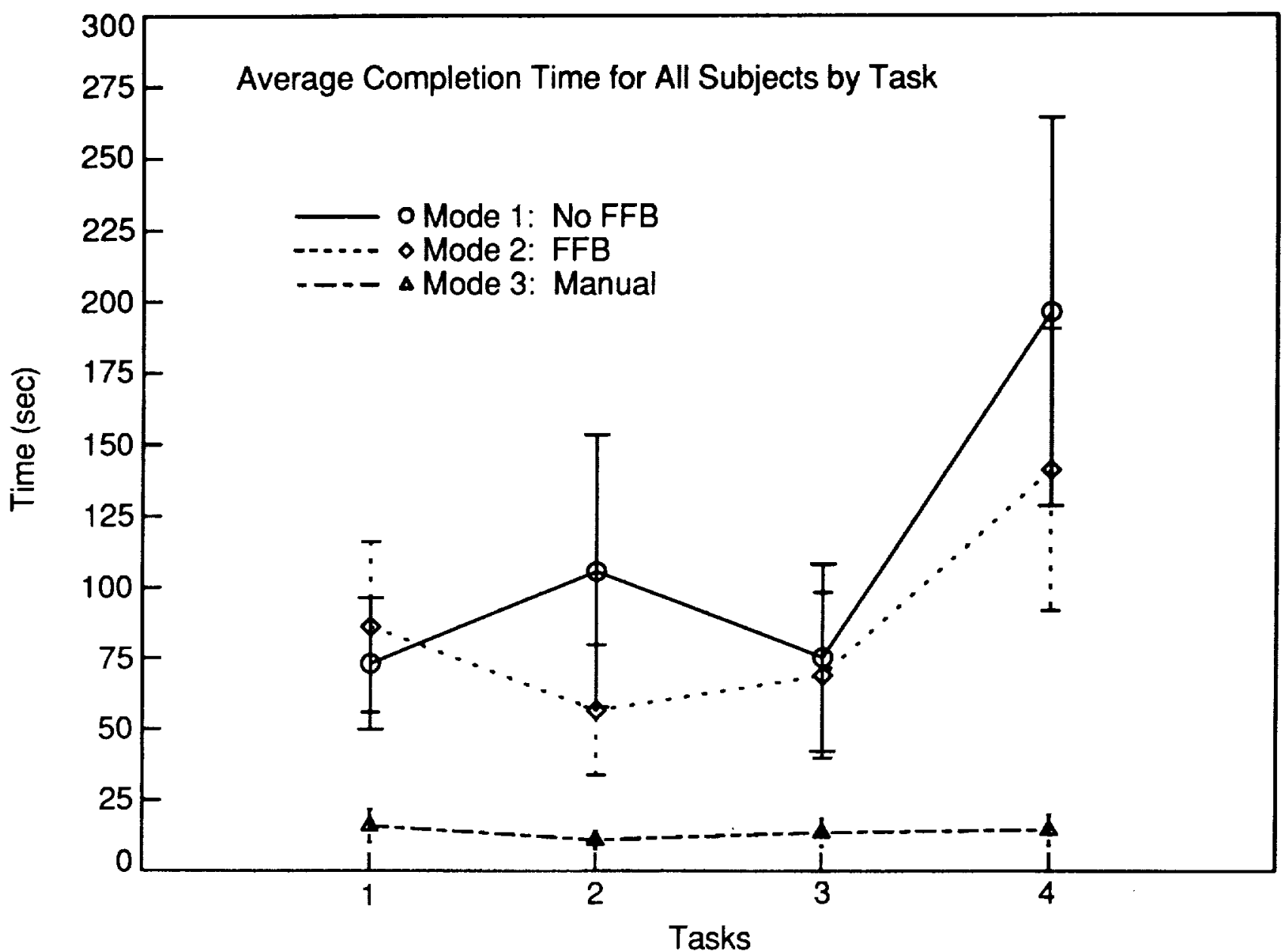

Tasks:

Task 1: Velcro Blocks Task 3: Electrical Connectors

Task 2: Peg in Hole Task 4: Bayonet Connector

Figure 21. Completion time plotted for each mode as a function of task. This is the same data as Figure 19, but replotted with the addition of data from the bayonet connector task. Diverging traces highlight the task dependence on the differential effect of force feedback. 


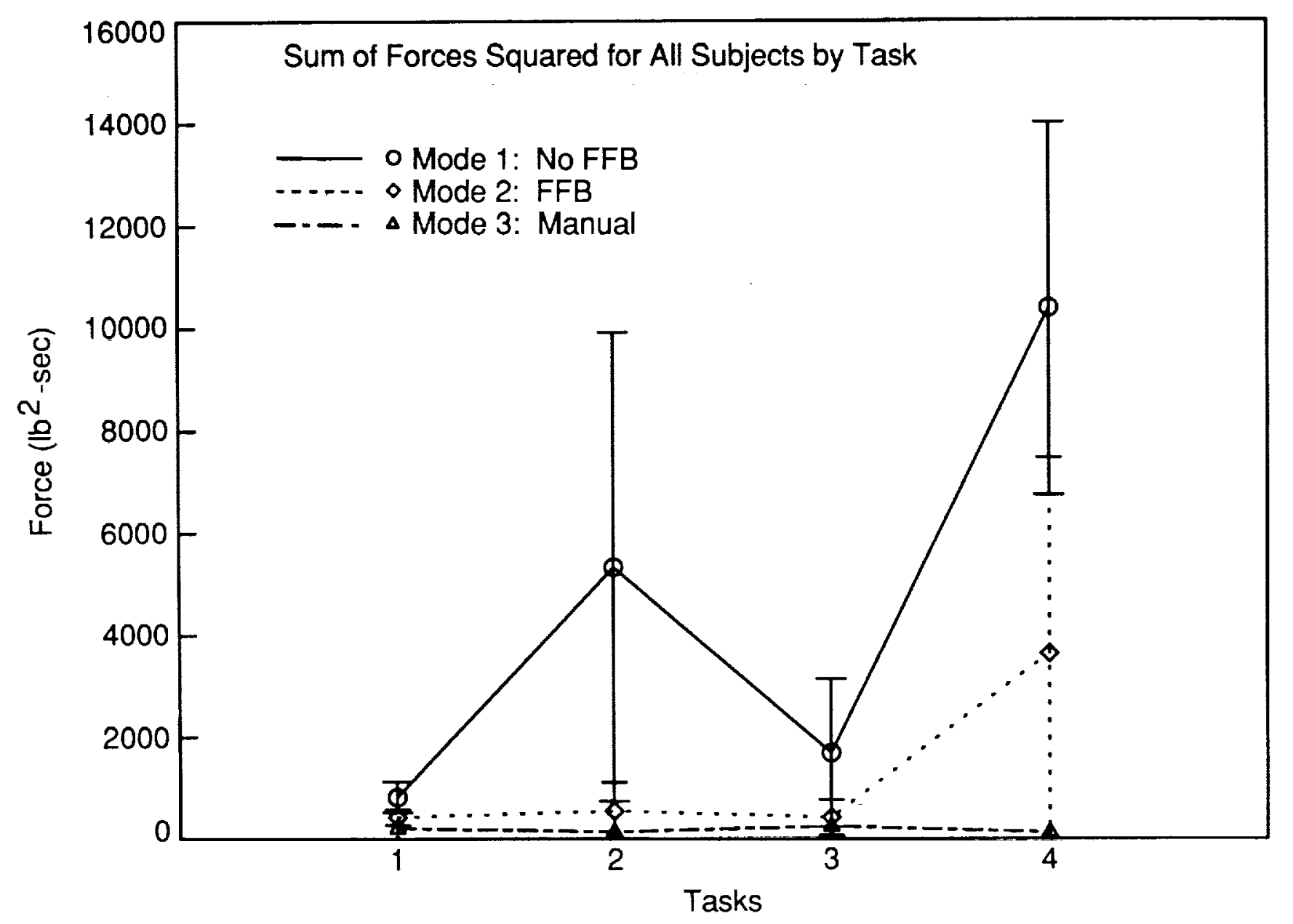

Tasks:

Task 1: Velcro Blocks Task 3: Electrical Connectors

Task 2: Peg in Hole Task 4: Bayonet Connector

Figure 22. Dependence of SOSF on task in the different control modes. 
The third task, electrical connectors, showed no change in completion time, but a significant drop in SOSF for all three connector types when force feedback was added. The last task, the bayonet connector, was similar in that there was a smaller change in completion time than in SOSF (see the last columns of Figures 23 and 24). The relative changes in completion time and SOSF with force feedback thus appeared to depend on the characteristics of the task.

Within an individual task, the results could be further broken down by subtask. This was done for the nine holes of the peg-in-hole matrix (Figures 23 and 24) (recall that the holes varied in diameter and in degree of chamfer). Referring to Figure 8 for dimensions, we see that diameter is constant for holes $1-3,4-6$, and 7-9. Thus a dependence of difficulty on hole diameter would be expected to show up as a stepwise decrease in performance (increase in completion time (CT) or SOSF) with hole number. Chamfer varied along the rows of the matrix. Thus, dependence of performance on chamfer would be expected to show as a sawtooth dependence of CT and SOSF on the hole number modulo 3 .

Neither of these dependencies was conclusively demonstrated by either the completion time or SOSF data. Thus, for the range of information measures covered by our experiment ( 6 to 8.6 bits), performance was remarkably independent of hole diameter or chamfer. Furthermore, the performance without force reflection was uncorrelated with the performance with force reflection.

When the electrical connectors task was broken down by subtask, we measured performance for the individual connector types (Figures 25 and 26). Completion time (Figure 25a) varied from 60 seconds ( $1 / 4$-in. and 3 -prong connectors) to 100 seconds (25-pin Cannon $\mathrm{D}$ connector). Improvement in completion time was slight or nonexistent with force feedback (triangles, dashed line). The force measure, however, showed substantial improvement for all three connectors. The force measure ranged from 800 to $2700 \mathrm{lb}^{2}$-sec for the connectors without force feedback to 200 to $700 \mathrm{lb}^{2}$-sec with force feedback. The improvement was greatest for the 25-pin D connector. Note that for these tasks, substantial improvement was shown in SOSF performance while there was little or no change in completion time.

The CT and SOSF performance measures are well defined not only for the whole task, but for the individual segments of the task as well. The segmentation algorithm described above was used on 150 repetitions of the peg-in-hole task. The results were completion times and SOSF measures for the individual segments: "movement," the free motion of the peg between hole and tap location; "insertion," the phase of the task from tip contact to grip release; "extraction," from gripper-peg contact to tip clearance; and "tap," momentary peg-to-board contact.

The CT was plotted for each of the segments comparing force-feedback and positioncontrol modes (all subjects, Figure 27). As expected, completion time for the movement and tap phases were unchanged by the addition of force feedback (at about 32 seconds and 2 seconds respectively). The insertion and extraction phases were accomplished markedly faster (11 vs. 31 seconds for insertion, 10 vs. 35 seconds for extraction) when force feedback 


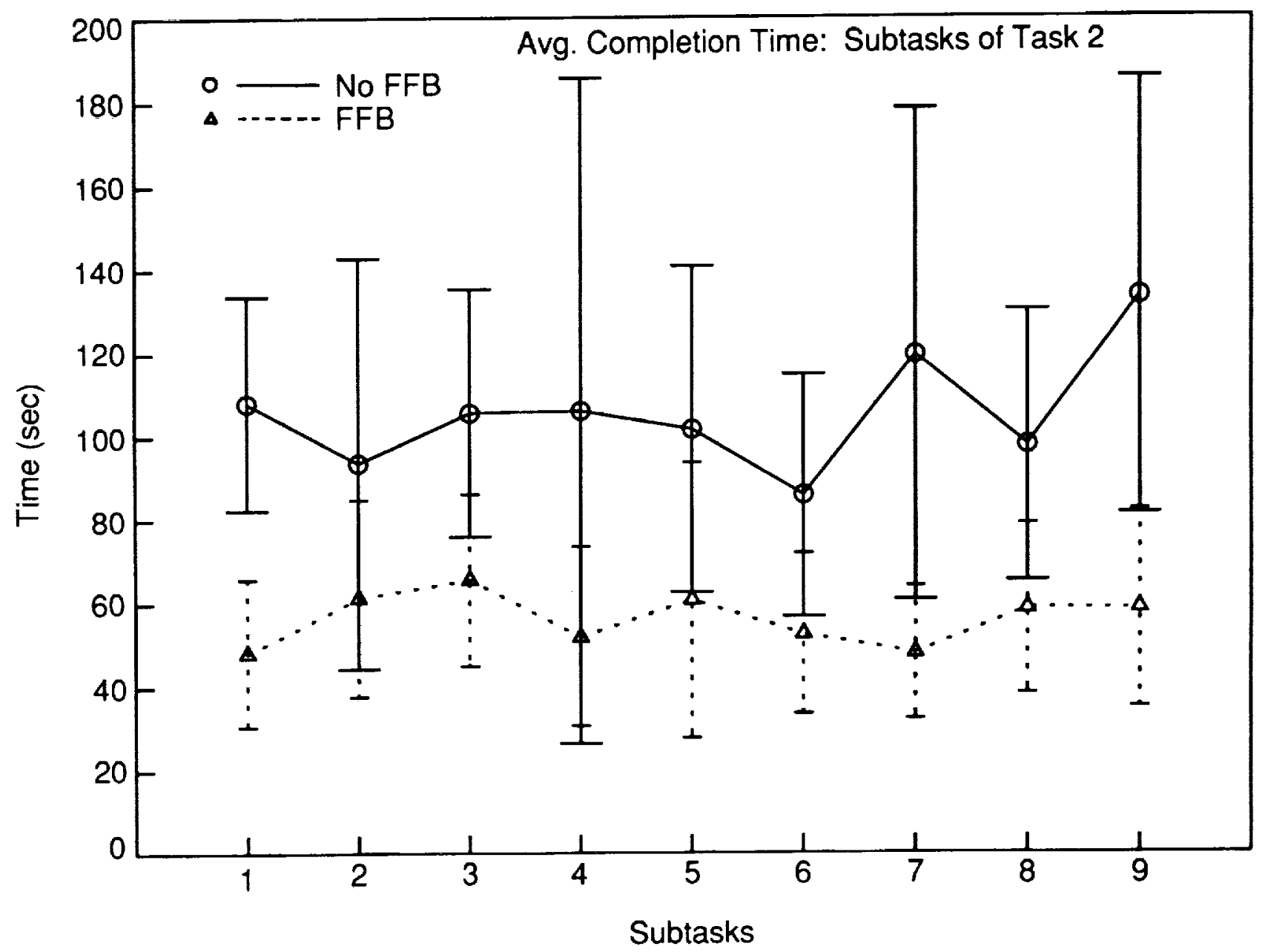

Figure 23. Completion time vs. hole number for the different tolerances in the peg-in-hole task. Hole geometry is defined in Figure 8. Dependence on hole diameter would show up as a stepwise increase in CT with jumps at \#4 and \#7. Dependence on hole chamfer would show up as a sawtooth dependence of CT on hole number modulo 3. Neither of these dependencies is conclusively demonstrated. Dependence would most likely show up if a wider range of hole tolerances had been used. 


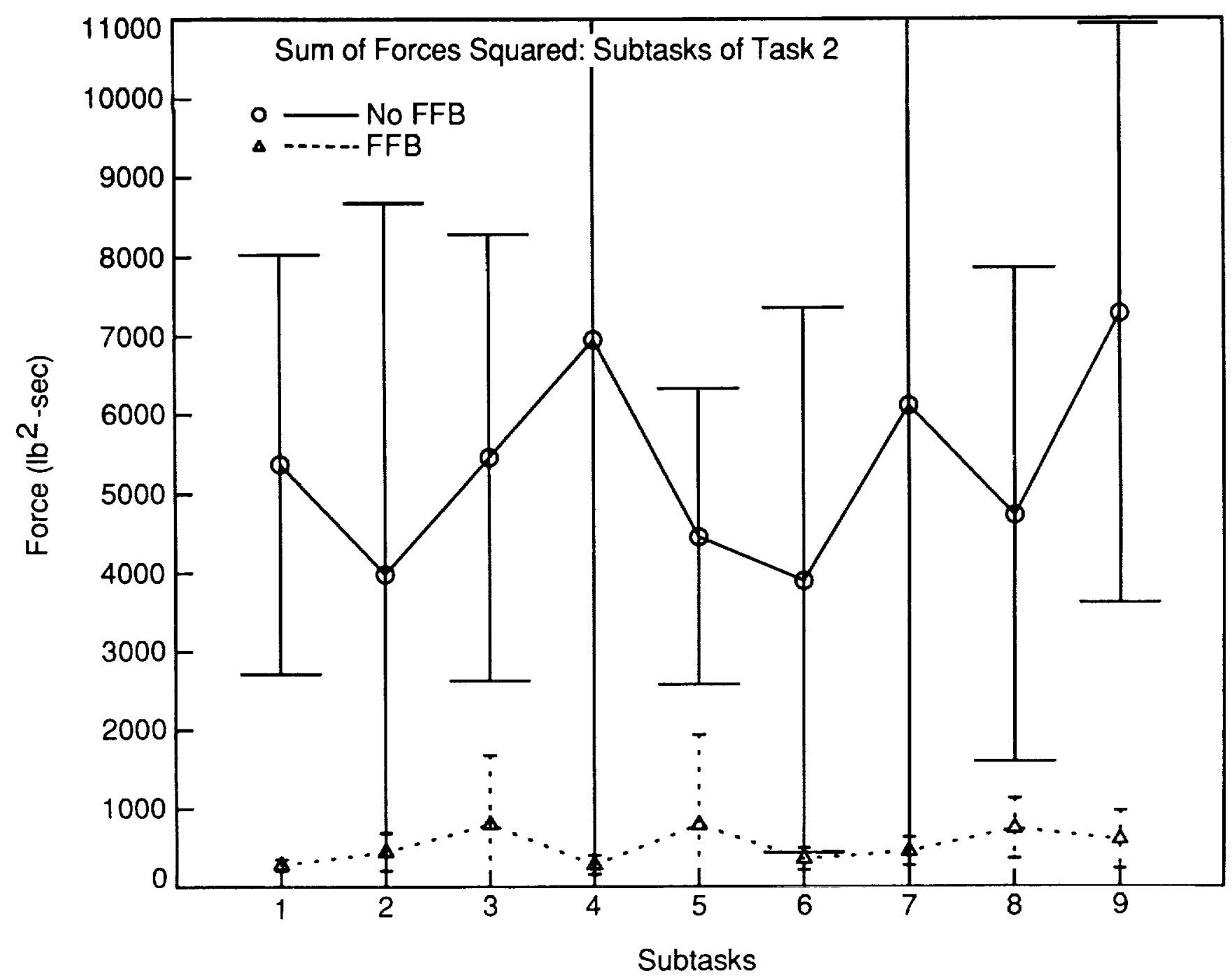

Figure 24. Dependence of SOSF on hole geometry for the peg-in-hole task. Results are similarly negative for the range of hole sizes (relatively high precision, see Figure 9) used. 


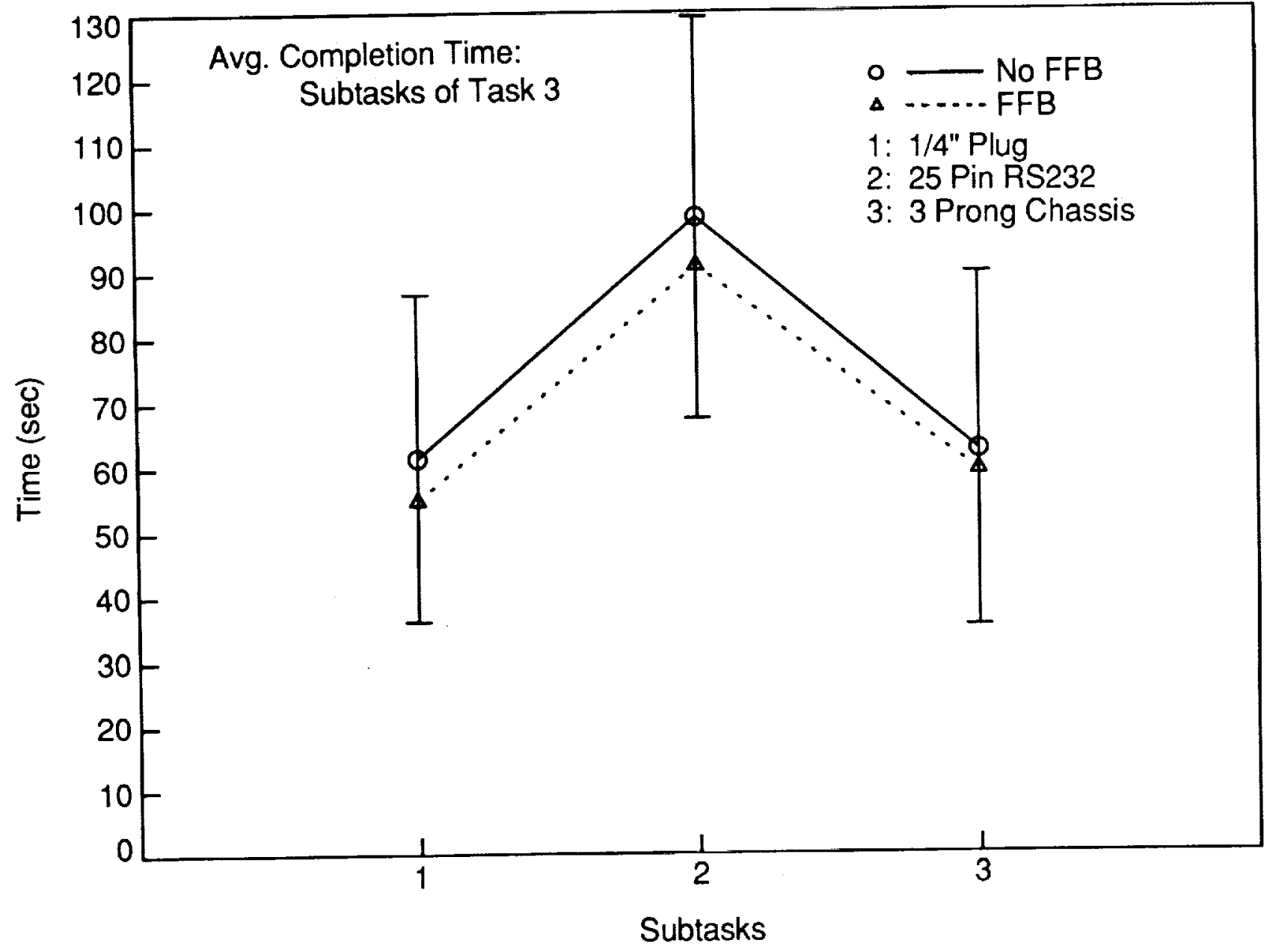

Figure 25. Completion time dependence on the connector type. Key to Subtasks: (1) 1/4-in. plug, (2) 25-pin DB25 RS232 connector, and (3) 3-prong chassis power connector. For these tasks, force feedback made no significant improvement in completion time. 


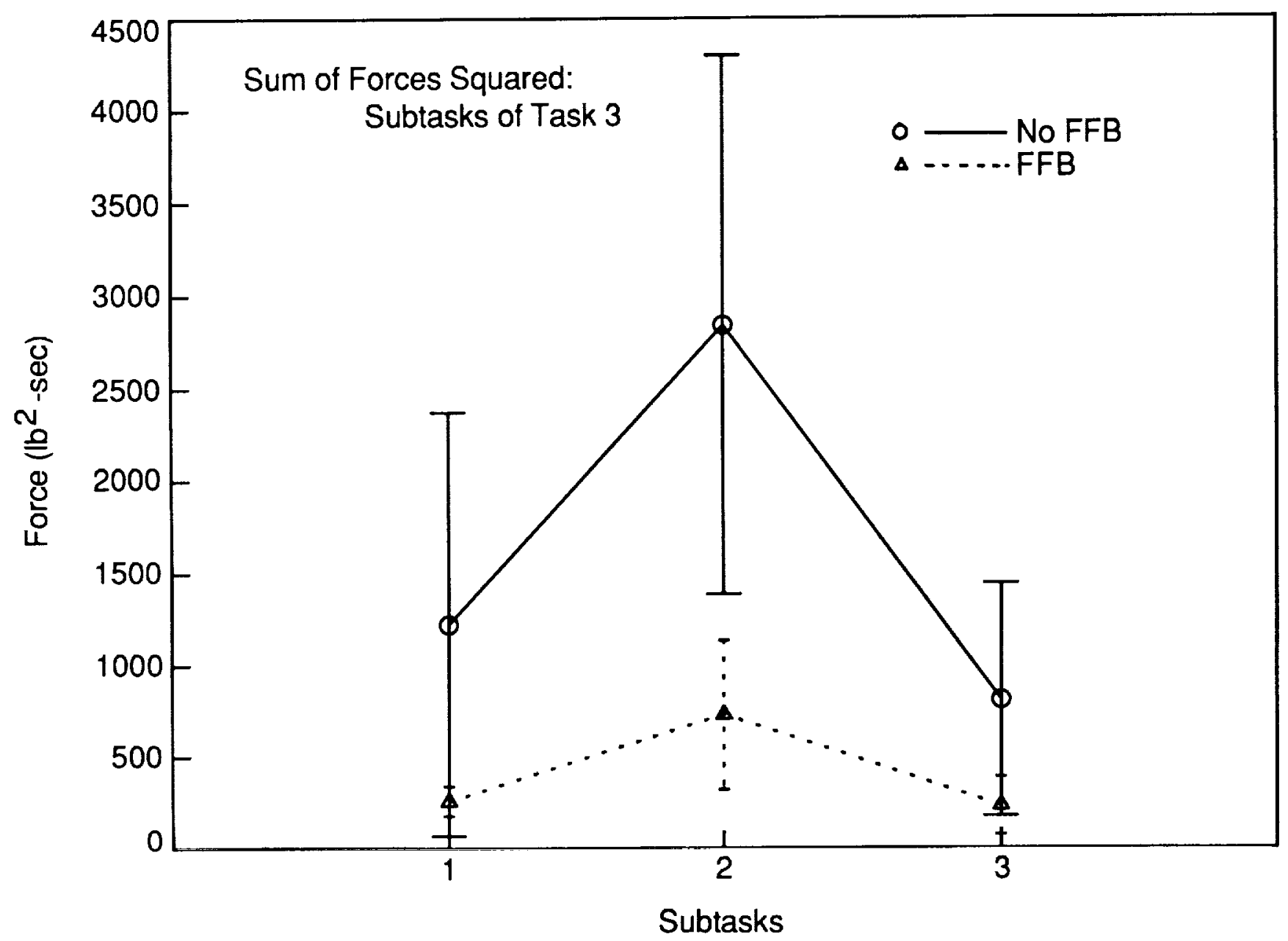

Figure 26. Force performance by connector type. SOSF is substantially reduced for all three connectors (see Figure 25 legend for key). The connector task appears to involve mostly precision alignment of the components, which is not speeded-up by force feedback. However, spurious forces, which are not required to mate the connectors but are generated in the course of exploratory manipulation, are minimized. 


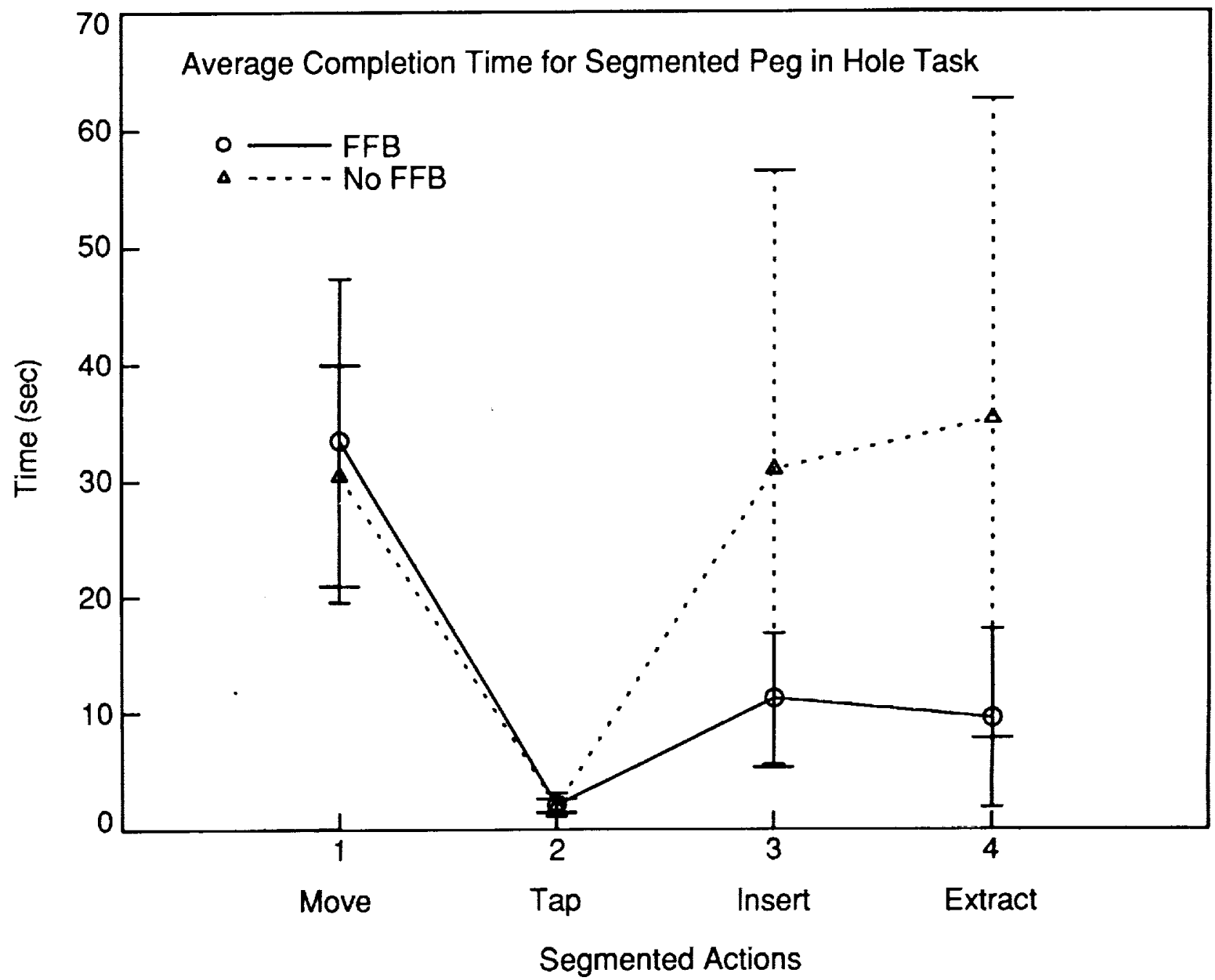

Figure 27. Changes in completion time by task phase for the peg-in-hole task. Force feedback substantially reduces the time to perform insertion and extraction without significantly affecting the time spent in translation or tapping. 
was present. Thus force feedback differentially affected different task phases.

The differential effect of force feedback dramatically changed the proportion of the total time spent in each of the task phases. Pie charts illustrate the mix of manipulation types in the task. Without force feedback (Figure 28) the task was dominated by the insertion and extraction phases: the task components involving energetic interaction with the environment. Together they accounted for $2 / 3$ (67 percent) of the total task time of 102 seconds. When force feedback was present (Figure 29) the total time was reduced to 55 seconds, and the proportion of time spent in energetic interaction was reduced to 37 percent. In contrast to the cases without force feedback, the dominant proportion of the total time with force feedback was consumed by the free-motion portions of the task.

Another variable of interest is the individual operator. Are there differences in performance between the different subjects? If so, are they consistent and predictable? Do different operators appear to follow distinct and identifiable strategies? Although this experiment was not designed to be a statistically rigorous study of these effects, certain regularities have emerged which inform speculation on these questions.

The task performance measures can be broken down and individually computed for each subject (Figures 30,31, and 32). On the basis of this limited sample, completion time (Figure 30) for the first three tasks was approximately the same (approximately $105 \mathrm{sec}$ ) for all five subjects except for subject 5 who was consistently faster (approximately $50 \mathrm{sec}$ ). The SOSF varied with the task but varied relatively little (i.e., by an amount comparable to one within-subject standard deviation) among subjects (Figure 31). Standard deviations in completion time appeared to be relatively invariant at about 30 seconds across the subjects for tasks 1-3. Standard deviation (s.d.) in completion time was also relatively constant at about 105 seconds for the difficult bayonet connector task. Error rates (Figure 32) varied markedly across the subjects as did the standard deviation of error rate from a high of 3 errors per repetition (s.d. $=2.5$ ) to a low of 1 error per repetition (s.d. $=0.5$ ). Subject 5 had the lowest (best) value in each of the three performance measures for most tasks.

\section{PRELIMINARY RESULTS: ADDITIONAL CONTROL MODES}

Future applications of telemanipulations will rely increasingly on the sharing of control between the human operator and, at first, limited autonomous functions. Beyond the three basic control modes reported above, Position Control, Kinesthetic Force Feedback, and Direct Manual, three additional modes (defined below) were evaluated in smaller experiments. These experiments were of smaller scale because of limitations of time and because some data were lost due to electromagnetic interference problems. (The number of data points for each of the six modes is shown in Figure 17.) The generality of these results is thus somewhat limited. However, they are reported because they still represent three repetitions of each subtask element, and are our first source of quantitative performance information on these advanced modes. 


\section{Time for Segmented Task using No FFB \\ Task \#2: Peg in Hole}

\section{Total Time: $105 \mathrm{sec}$}

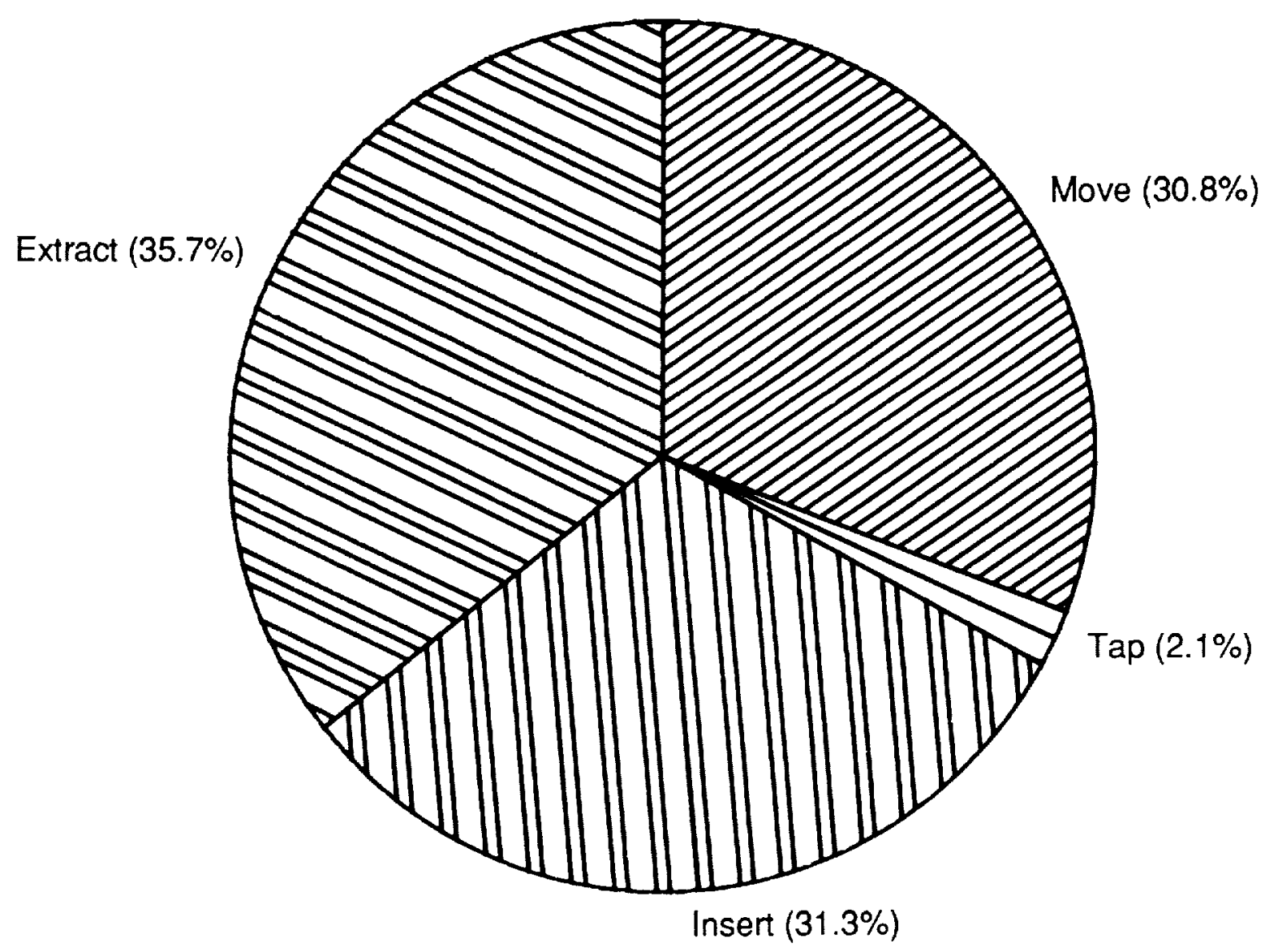

Figure 28. Task phase breakdown with Position Control. Without force feedback, total completion time is approximately evenly divided between moving, insertion, and extraction in the peg-in-hole task. The data are the same as the dotted line of Figure 27. 


\section{Time for Segmented Task using FFB \\ Task \#2: Peg in Hole}

\section{Total Time: $55 \mathrm{sec}$}

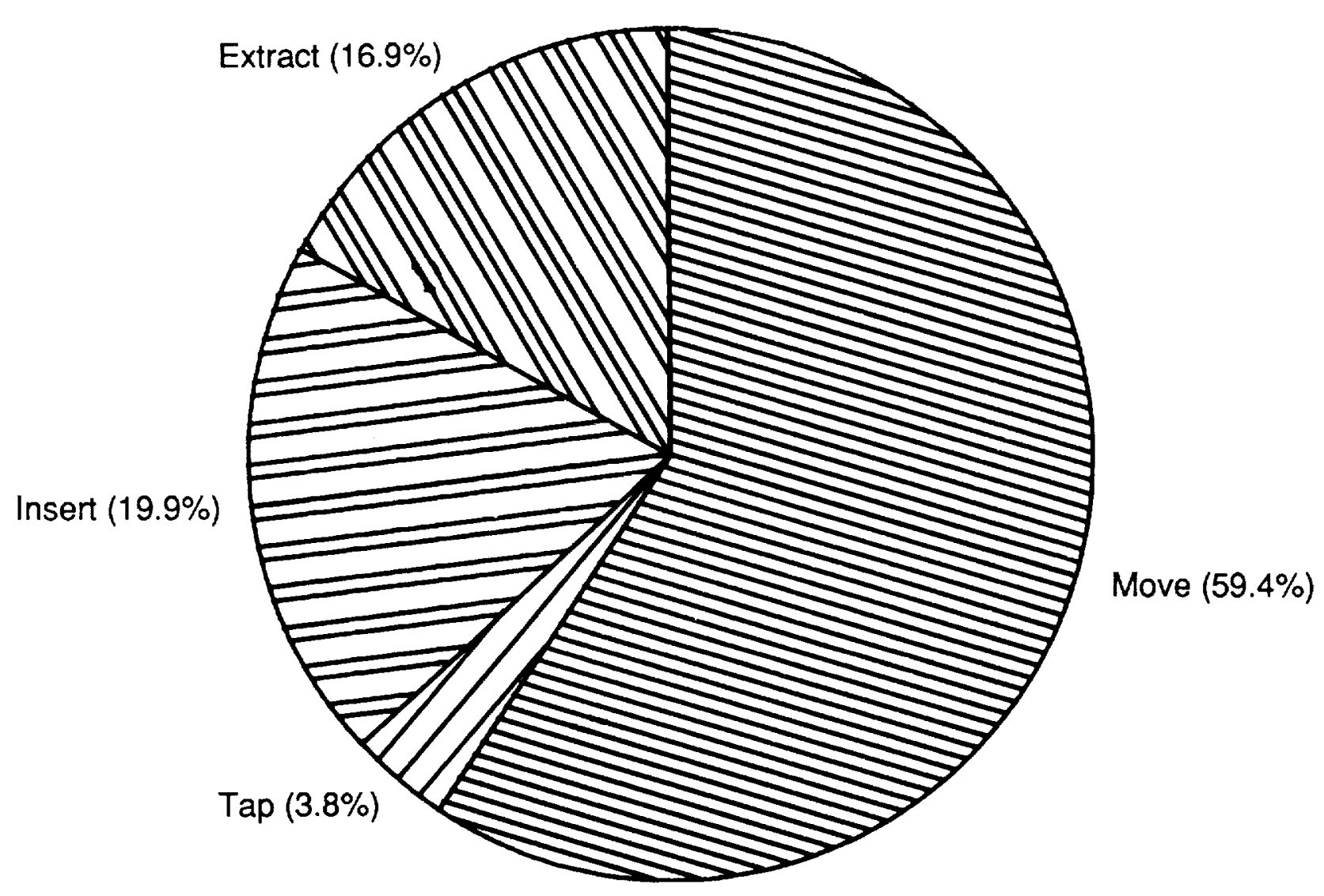

Figure 29. Task phase breakdown with KFF. When the task is performed with force feedback, total time drops from $105 \mathrm{sec}$ to $55 \mathrm{sec}$. As a result, the completion time is dominated by the translation phases of the task. Changing the level of manipulation capability redefines the task weights. 


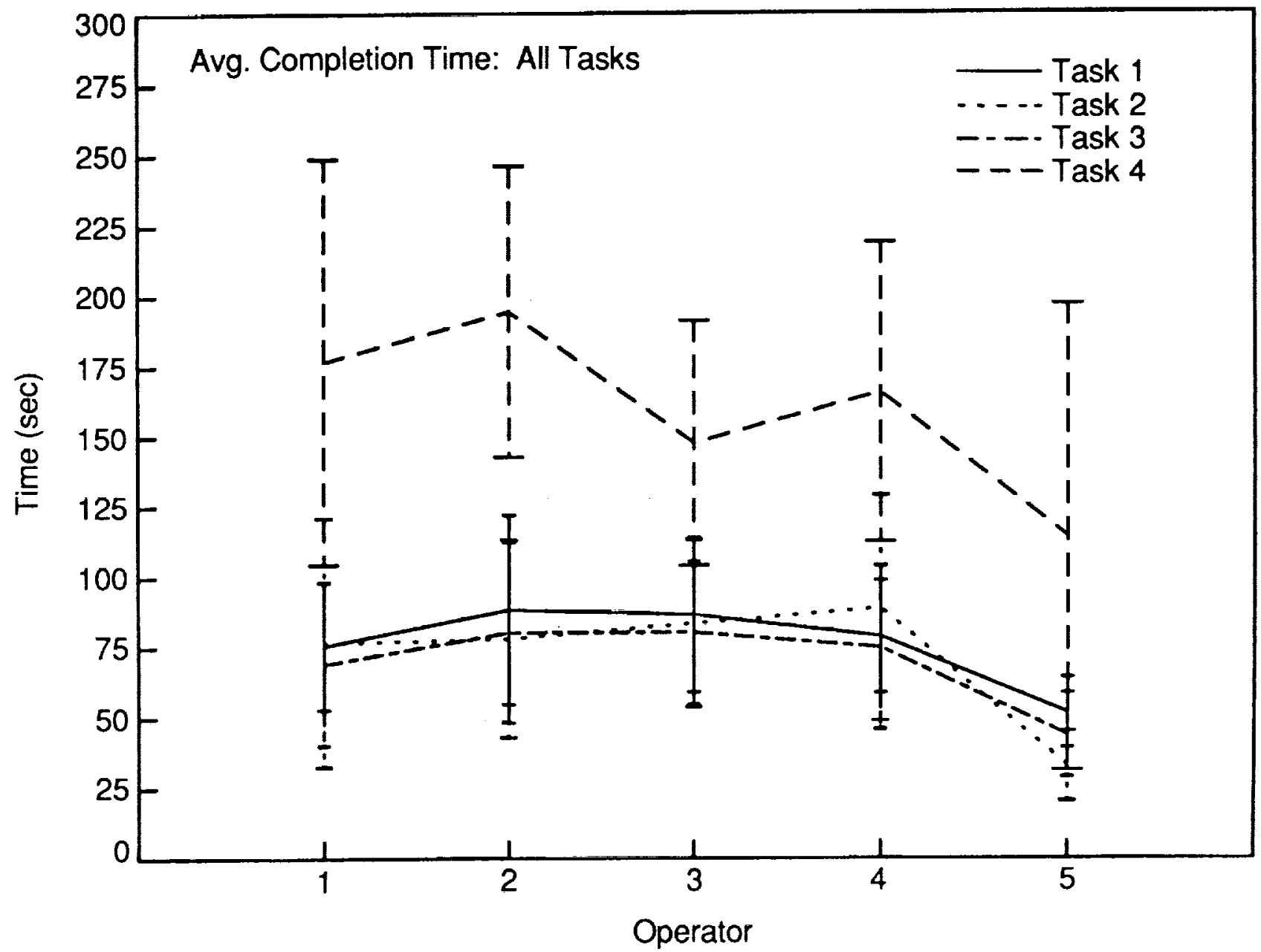

Figure 30. Inter-operator variation. Completion time was relatively constant among the test operators for the first three tasks. Variation was greater for the difficult bayonet connector task. 


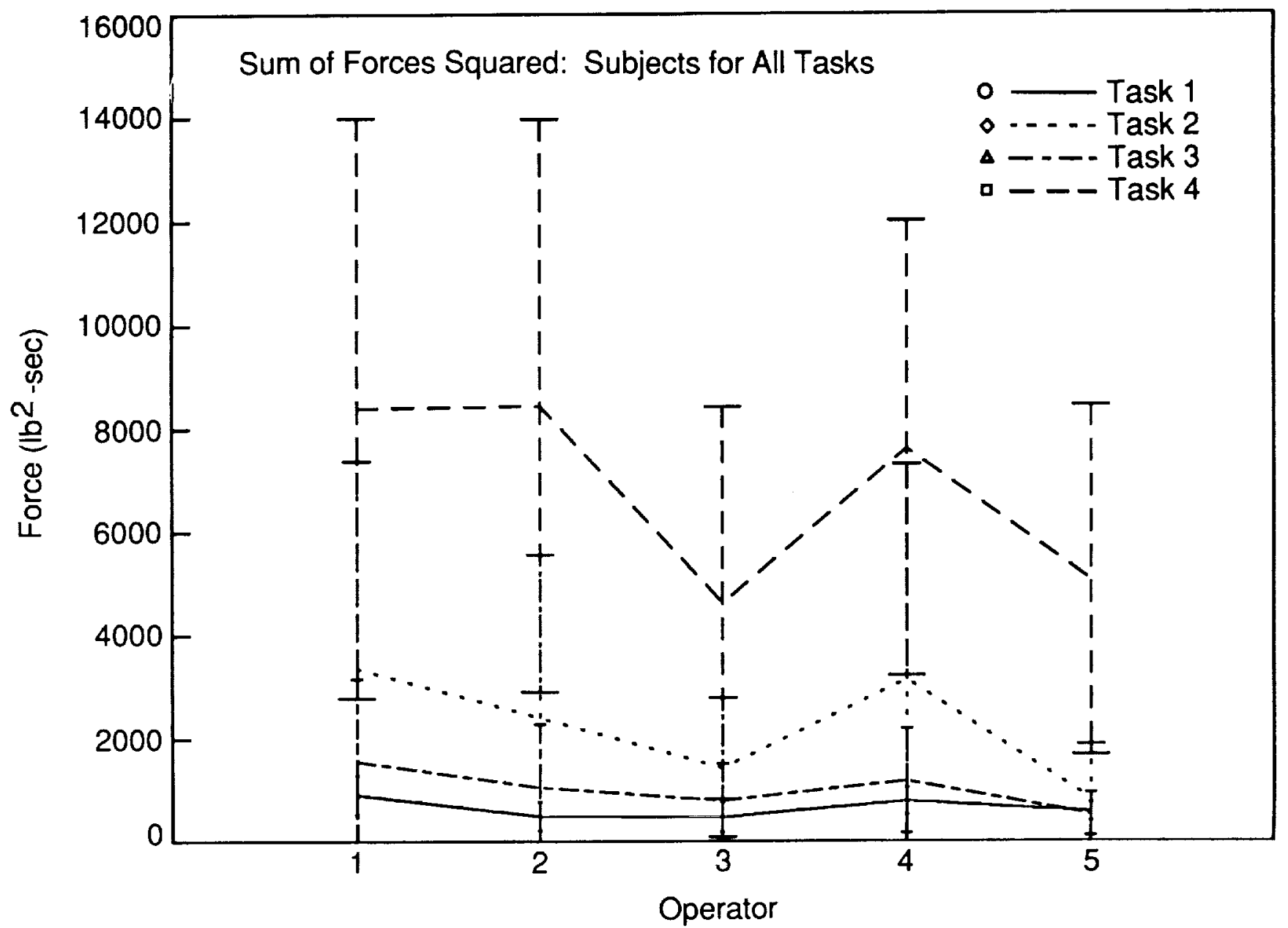

Figure 31. SOSF variation among test operators was greater than completion time. 


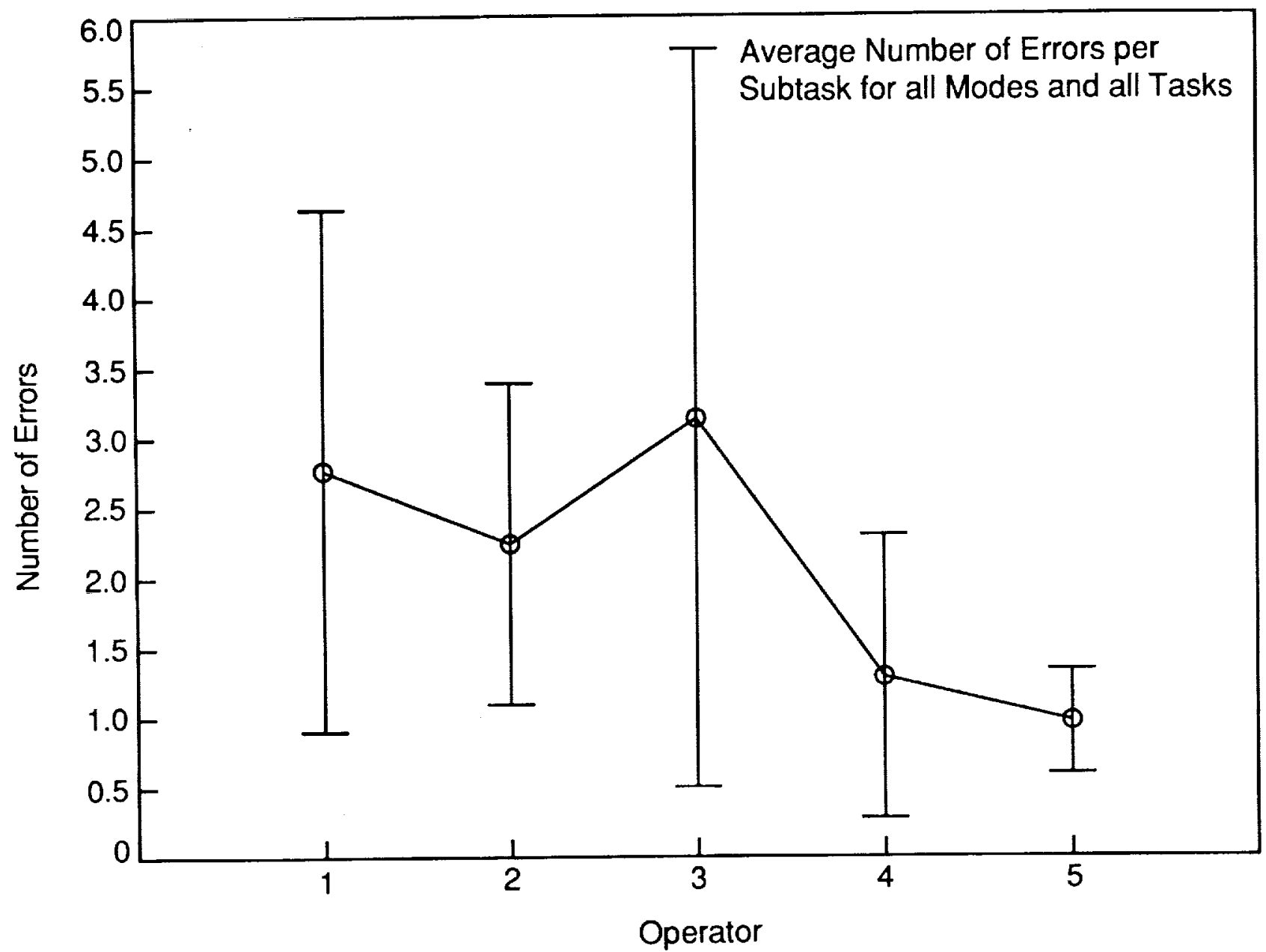

Figure 32. Error rate showed the greatest variation among the test operators. 
The three additional modes were repeated three times each for the complete task set by at least one subject. One relatively primitive mode, "Position Control with Visual Force/Torque Display," and two modes of shared control, "Shared Force Accommodation" and "Hybrid Force Accommodation," were tested by at least one subject. These modes are defined above in the "Capabilities" section.

Two subjects whose performance was quite similar in the modes for which both data sets exist were compared in all six modes for all of the first three tasks. Completion time performance (Figure 34) is slightly improved ( 83 seconds vs. 100 seconds) with the use of the graphical force display (significance not tested) although not by as much as kinesthetic force feedback (mode 3). "Shared Force Accommodation" mode resulted in the lowest overall completion time of any of the telemanipulated modes (approximately 44 seconds). "Hybrid Force Accommodation" in which both subjects had approximately equal performance, showed an increase in completion time compared to kinesthetic force feedback from about 70 to 80 seconds (significance not tested). SOSF performance (Figure 35) showed larger differences in the same pattern.

The third performance measure, task error rate (Figure 36) varied in a similar pattern (no data were available for the hybrid mode). In this plot, data from each subject were averaged for each point. Thus, for mode 1 , data from all subjects are included, whereas for modes 2, 4, and 5 (see Figure 36), only one subject is represented. For completeness, completion time and SOSF are broken down by task type (Figures 37 and 38). These data are averaged as described for task error rate. 


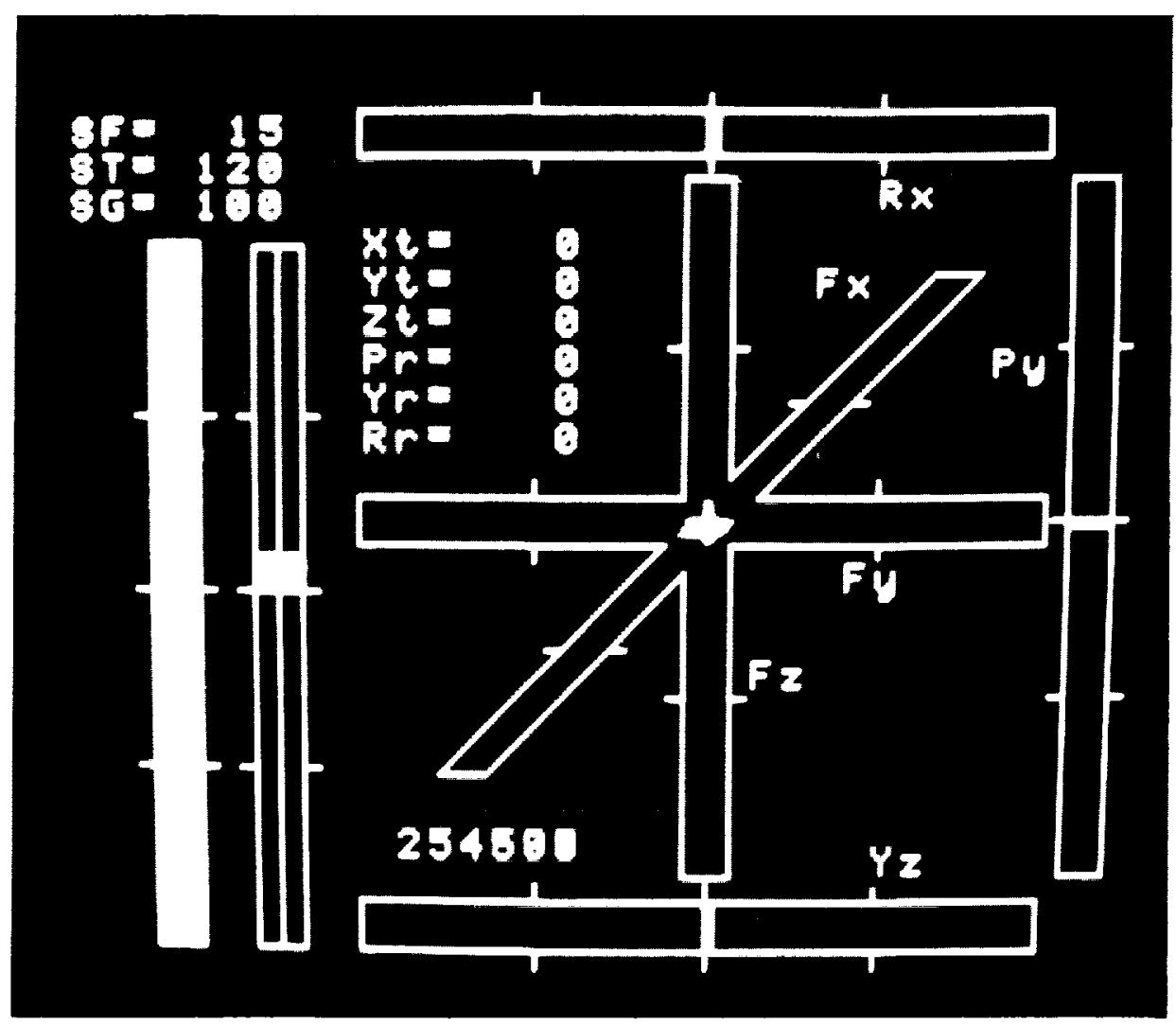

Figure 33. Video display of force-torque information. The bar graphs form a pseudo perspective view of the hand coordinate frame. The diagonal bar represents the $X$ direction (in and out of screen). Bars at upper, right, and lower edges represent the moments around the $\mathrm{X}, \mathrm{Y}$, and $\mathrm{Z}$ axes respectively. Bars on right represent finger opening and clamping forces. 


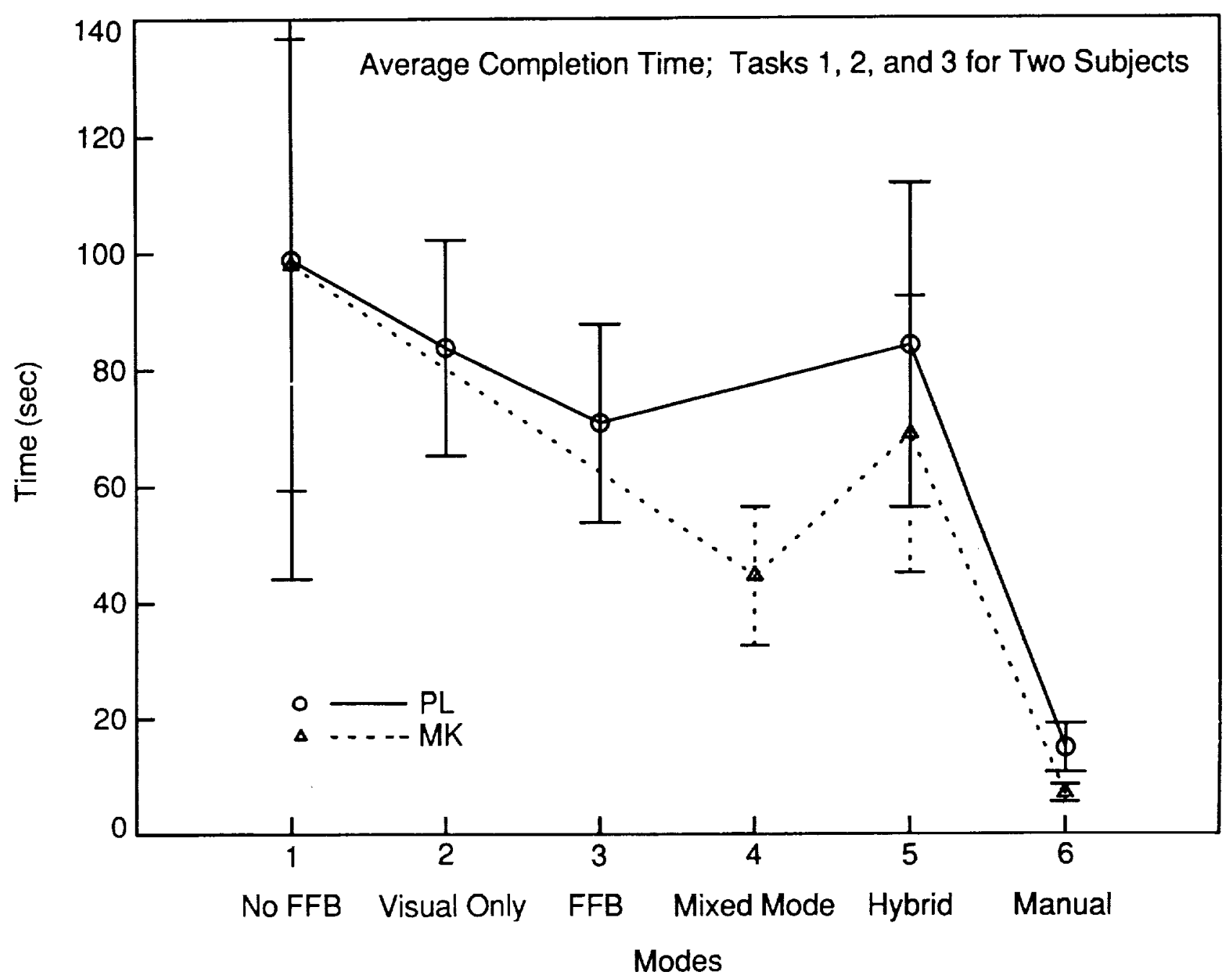

Figure 34. Completion time for additional modes. Preliminary experiments were performed with three additional modes: visual force display, and two shared control modes, "Shared Force Accommodation" and "Hybrid Force Accommodation" (see text). Completion time with "Shared Force Accommodation" was the best of any telerobotic mode tested. Generally speaking, performance improved as the level of capability increased with the exception of "Hybrid Force Accommodation" mode, which did not improve performance. Because of the limited amount of data, these results are not statistically conclusive. 


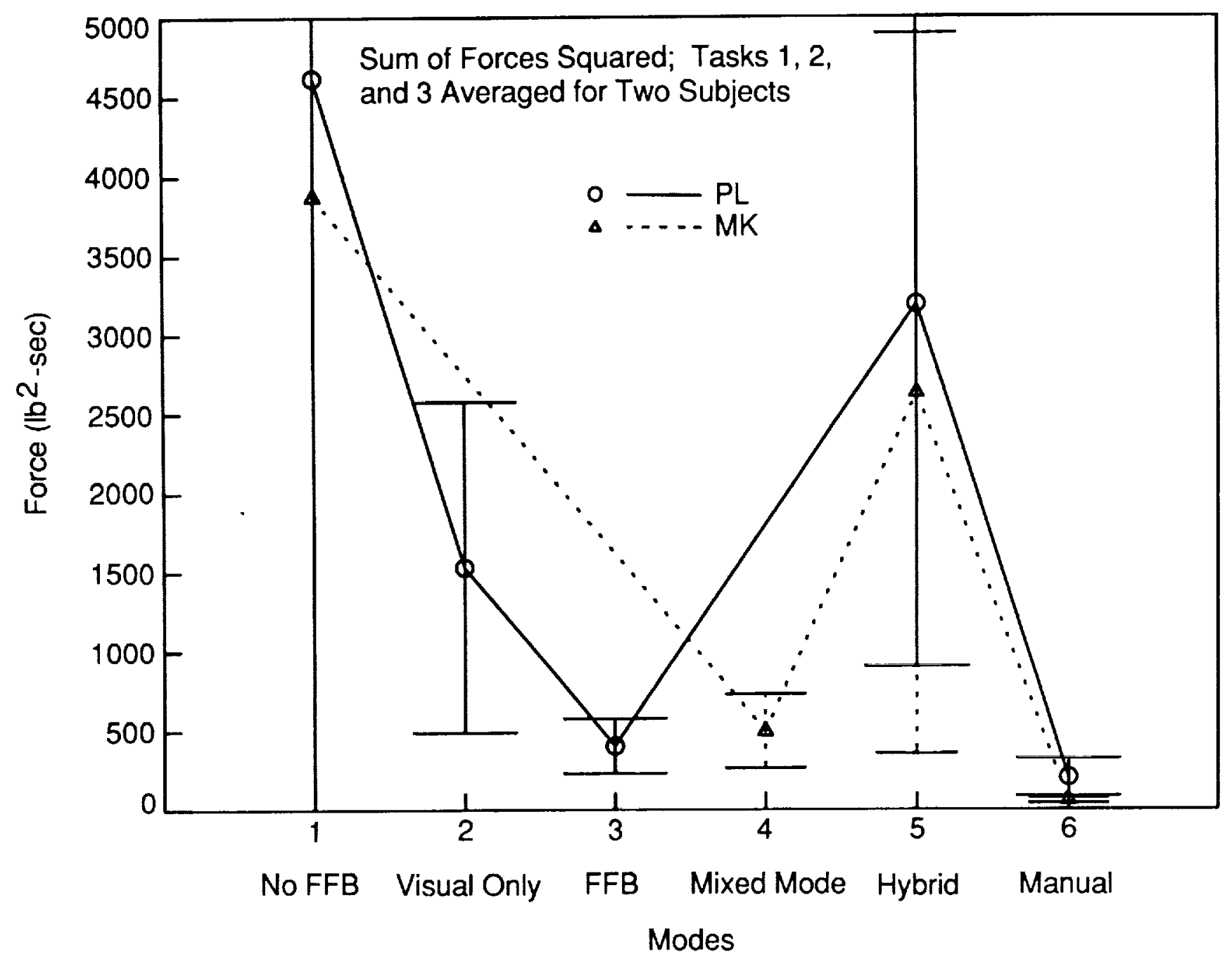

Figure 35. SOSF with additional control modes. The force control performance shows larger changes in the same general pattern as completion time. 


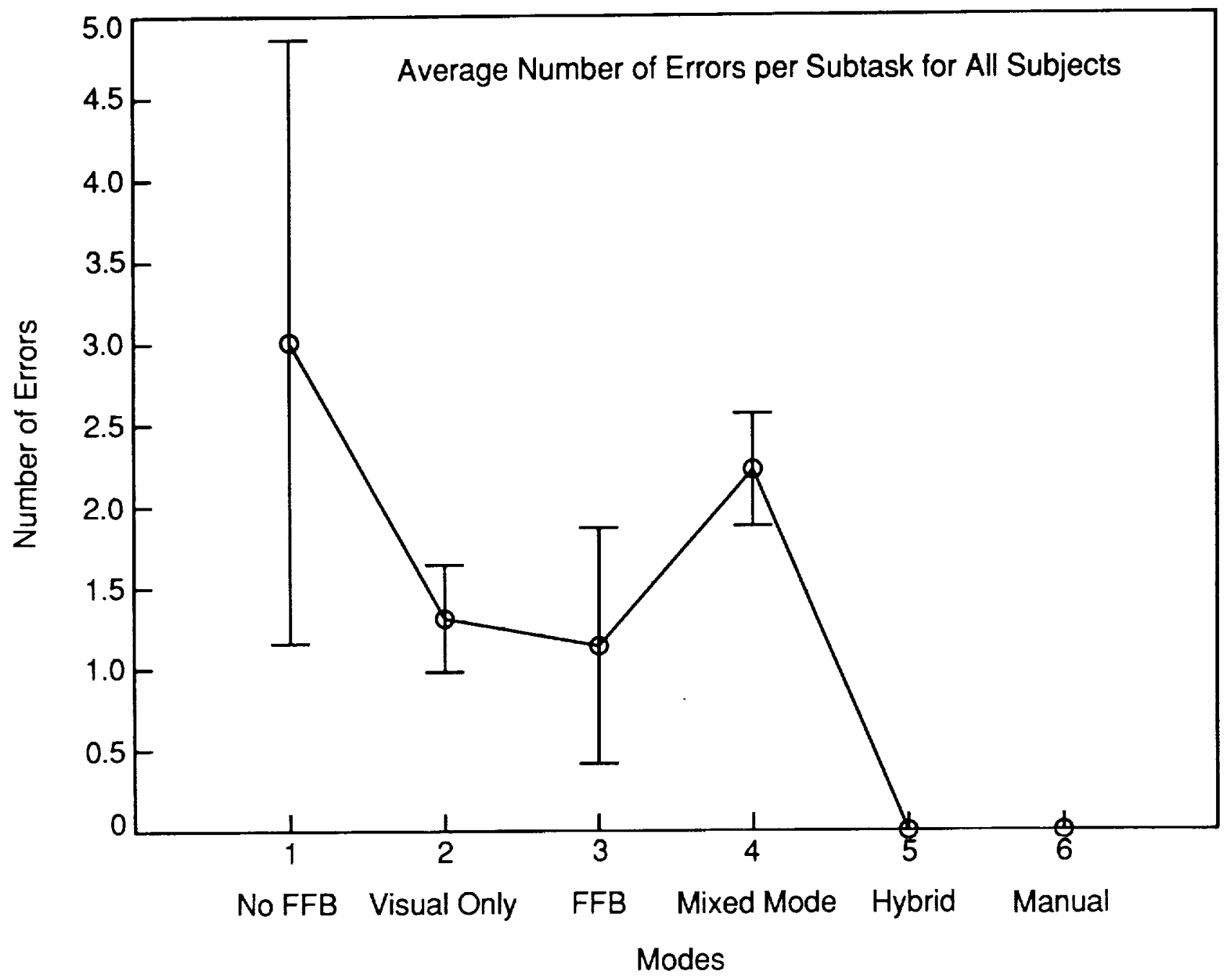

Figure 36. Number of task errors. Visual force feedback (mode 2) has a larger effect on error rate than CT or SOSF. Data for mode 5 are missing. Bare-handed human operator ("mode" 6) made no errors. 


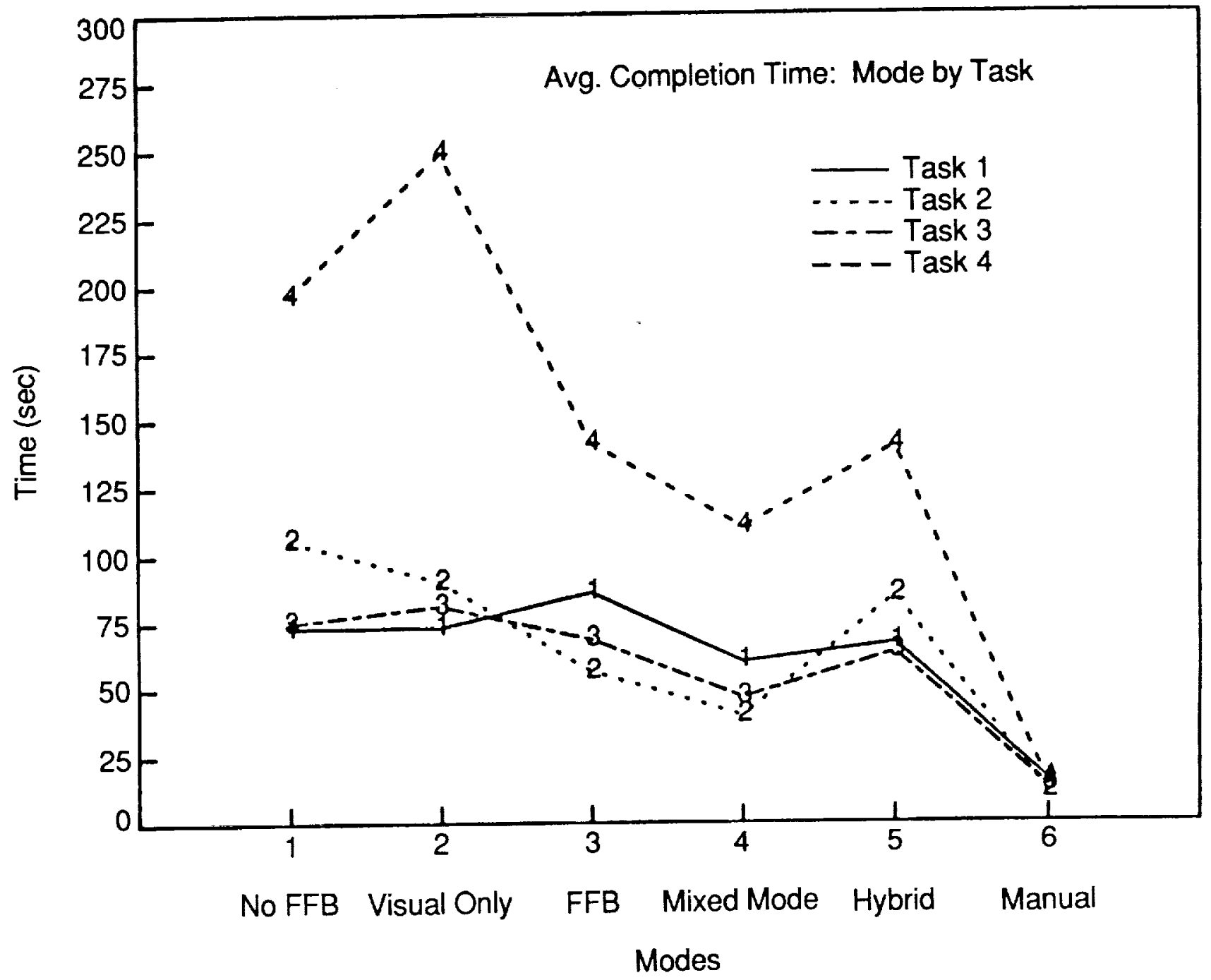

Figure 37. Completion time vs. mode. The added modes affected each task differently. 


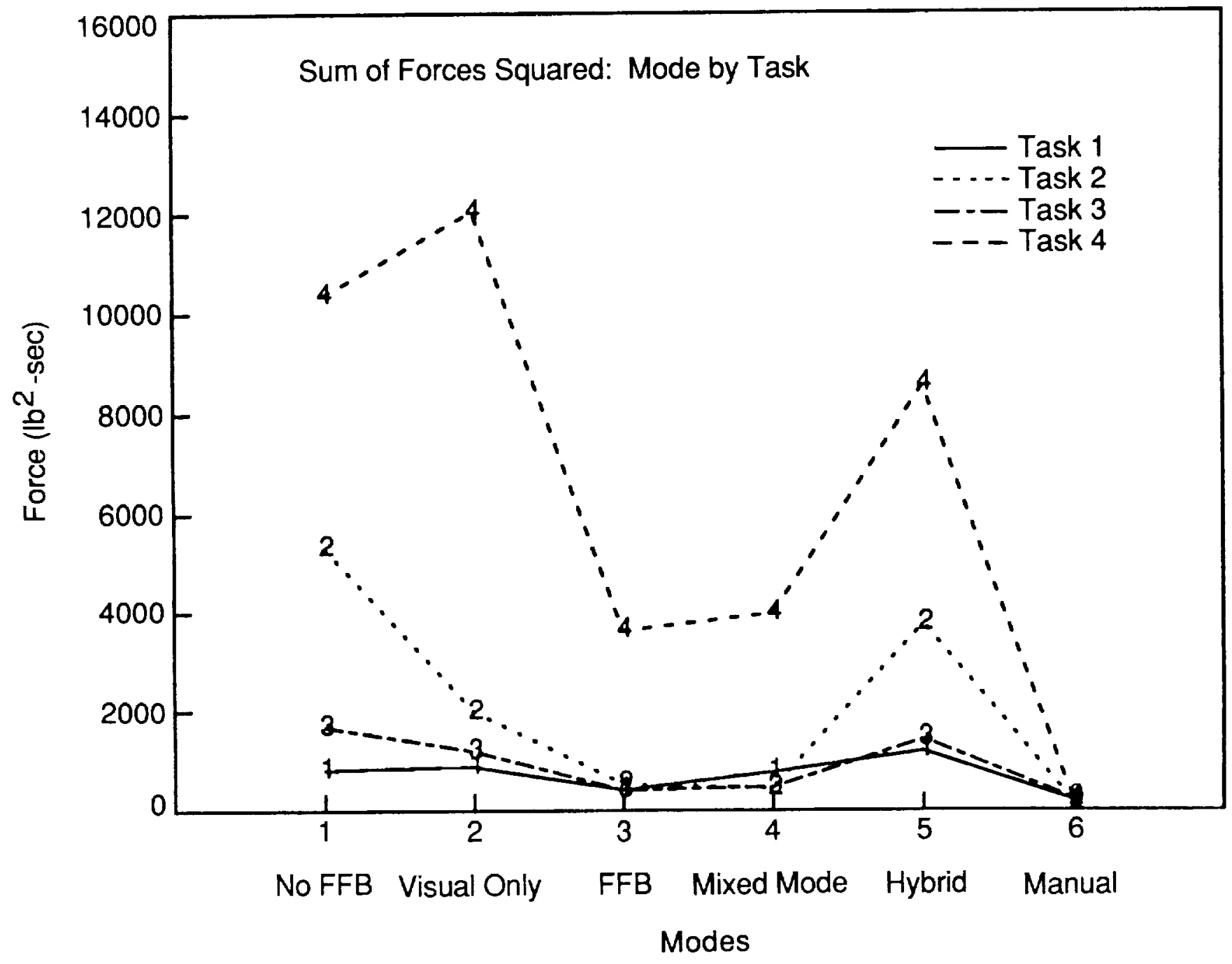

Figure 38. SOSF performance as a function of control mode. 


\section{DISCUSSION}

This study has characterized an advanced-telemanipulation-system prototype in quantitative terms relevant to application planning. As yet, only recent theoretical developments in the modeling of bilateral manipulation systems (Hannaford 1989, Raju 1988) can provide a full characterization of the system's performance independent of the operator, and it remains to relate these performance measures to performance on actual tasks. Thus the approach was to use multiple test operators performing an array of different tasks under the various operating conditions.

\section{SYSTEM AND CONTROL MODES}

One of the unique features of the ESAB system is its astonishing number of possible control modes. Since each of the six task-space axes can be set into one of ten modes independently, one million combinations are generated. In addition, several parameter values must be specified for each axis. So far we have used only simple heuristics to choose among the huge number of ways the system can be controlled. The preliminary evaluation of two of these possible modes (Figures 34 through 36) showed that "Shared Force Accommodation" - a shared control mode in which Kinesthetic Force Feedback Control was used in translation and rotation axes and Local Force Accommodation used in orientation axes - resulted in improved performance (relative to force-reflecting teleoperation), and that "Hybrid Force Accommodation" - in which pitch and yaw axes were locally controlled and translation was operator controlled without force feedback - resulted in poor performance. This demonstrates that emerging forms of shared telerobotic control must be carefully selected and matched to the task. With the present state of knowledge of manipulation, we have no way of knowing at this point whether among the thousands of other possible modes there is one which enables even better performance than observed under "Shared Force Accommodation."

It is reasonable that visual feedback of force/torque information should result in a performance level intermediate between Position Control and Force Feedback (Figures 34 through 36). However, an earlier study (Hannaford 1987) did not find improvement. Among the differences between the studies which might explain this are the use of rate control in the earlier study vs. position control here, better procedures and training for the test operators, and the improved dynamics of the mechanism and control system used in the current study.

\section{MECHANICS AND STABILITY}

One of the key performance issues in design of active telemanipulators is stability. The stability issue arises when forward position commands and kinesthetic force feedback combine to form a closed-loop system encompassing the human operator, hand controller, robot manipulator, and environment. Various studies (Hannaford \& Anderson 1987, Hannaford \& Fiorini 1988, Anderson \& Spong 1988, Hannaford 1989) have put forward analytical, computational, and experimental models of this system and its dynamics. This work was consistent with operational observations that stability during manipulator contact was in- 
versely related to force-feedback scale. This parameter, the force-feedback scale factor, is referred to as $h 12$ in the hybrid two-port nomenclature described in Hannaford (1989). This scale factor could be reduced to obtain stable operation. In the experiments reported here, $h 12$ was 0.1 so that only $1 \mathrm{lbf}$ was applied to the operator for each $10 \mathrm{lbf}$ applied to the manipulator tip. However, the cost of the stability obtained in this manner was not only reduction of the kinesthetic feedback of force information, but also, effective magnification of the force perception threshold of the man-machine system. This is so because the mechanism of the force-reflecting hand controller has significant friction levels between the actuators and the handgrip which effectively absorb all force signals below a certain force level, Fmin. The human operator has some perceptual threshold for forces, Fth. Thus, the man-machine perception threshold is

$$
T m m=\frac{(F \min +F t h)}{h 12}
$$

in the sense that any force applied to the slave will not be felt if its magnitude is below Tmm. In our case, the effective man-machine threshold is then $10(F \min +F t h)$. For mechanisms available today, $F$ min can be on the order of $8 \mathrm{oz}$, which in the above example, neglecting the human operator threshold, scales to an effective deadband to force sensitivity of $5 \mathrm{lb}$. Reducing this threshold is a major challenge in telemanipulation system design.

\section{TASKS}

The tasks studied fell into one of two broad classes, "generic" and "application" tasks.

Although the main generic task (peg-in-hole) is a classical task in the robotics lab, its relationship to the "real world" is illustrated by the relationship of the tolerances used in our task board to industrial parts-mating practice (Figure 9). The peg and hole sizes reported here as well as those used by Hill (1977) (open triangle in the figure) are squarely in the range of "General Machine Bearing Practice." One difference between the peg-inhole task and the industrial parts illustrated is that the pegs are long compared to their diameter and thus have large contact surfaces. As a result, near the point where the tip of the pcg has just entered the hole, the bending moment can be quite large, causing binding problems at that point in the task (as well as near extraction). Thus the parts-mating capability demonstrated in this and other studies may be extendible to tighter tolerances for parts with lower ratios of length to diameter.

The electrical connectors qualify as application tasks because they are stock items unmodified for the experiments. Despite the occasionally high contact forces encountered in some of the experimental conditions and the large number of repetitions, none of these stock electrical connectors suffered damage from the robotic operations. In all of the connector tasks, the task board contained the female connector and the male was manipulated by the robot.

In both the velcro and electrical connector tasks, and to a lesser extent in the bayonet connector task, kinesthetic force feedback substantially reduced SOSF but did not 
substantially affect completion time. This indicated that these tasks are primarily precise positioning tasks and that while force feedback did not aid task performance in terms of time, extraneous forces arising from contact with the task board were reduced by the kinesthetic force feedback.

\section{SUBJECTS}

Variation among subjects was surprisingly slight. Their backgrounds were similar (engineering students or recent graduates) except for one who was a physical education major with training in gymnastics and coaching. This subject showed the best overall performance by each of the measures. This apparent correlation between performance and prior academic background might suggest that potential operators be grouped into classes based on interests or aptitudes and that these groupings might predict relative levels of telemanipulation performance. Of course this must be tested with statistically significant numbers of test operators.

We cannot conclude that there was any predictive value to the "motor control" tests the subjects performed prior to the telemanpulation experiments. Although further experimentation is under way in this area, new preliminary results tend to confirm this negative finding. Of course, some other test may be found which will reliably predict telemanipulation performance. The personality-type hypothesis described above needs to be further tested. If it appcars to hold, then surveys or personality inventory tests might prove useful.

\section{CONCLUSIONS AND RECOMMENDATIONS}

The main results of this study are that manipulation performance can be quantified along at least three dimensions, and that these measures showed task dependent increases in performance as system capability was increased. The unaided human operator was placed at the highest extreme of the scale of manipulation capability because presentday remote-manipulation systems all introduce substantial distortions and movement constraints between the operator and task, and thereby reduce performance. It is expected to take much time before telemanipulation performance will equal that of the bare-handed human. This study has quantified the ground remaining to be covered. In striving to achieve this level of performance, these results should be interpreted as data supporting more advanced modes of telemanipulation than are presently in widespread use. Although addition of kinesthetic force fcedback is of substantial help in moving performance towards the extreme demonstrated by the bare-handed human, more advanced shared-control modes offer further improvements. It is also likely that further improvements in the fidelity of kinesthetic force feedback will substantially improve performance.

As was found in the studies mentioned in the introduction, this work supports the idea that multiple measures of performance must be used to characterize telemanipulation. This is illustrated by cases such as the electrical connector tasks, in which kinesthetic force feedback did not substantially affect completion time, but did sharply reduce extraneous forces. In this case the precision positioning demands of the task were not aided by force feedback, but potentially damaging forces could not be controlled without it. 


\section{FUTURE WORK}

Experimentation using similar tasks and methodology in which time delay is added between master and slave sides is already under way. These experiments will be useful for systems applications such as the Space Station, in which interfacing constraints add time delays on the order of $50 \mathrm{msec}$, as well as future applications such as ground-based teleoperation in low earth orbit, in which delays from 2 to 4 seconds will be involved.

Other plans include expansion to dual arm capability and more complex tasks such as module exchange and assembly. The direct human control data should be supplemented with data from subjects wearing pressurized space gloves and calibrated against experimental EVA. 


\section{REFERENCES}

Bejczy, A.K. and M. Handlykken. 1981. "Experimental Results with a Six-Degree-of-Freedom Force Reflecting Hand Controller," Proceedings of the 17th Annual Conference on Manual Control, Los Angeles, CA.

Bejczy, A.K. and J.K. Salisbury. 1983. "Kinesthetic Coupling For Remote Manipulators," CIME (Computers in Mechanical Engineering), vol. 2, no. 1, pp. 48-62.

Bejczy, A.K., B. Hannaford, and Z. Szakaly. 1988. "Multi-Mode Manual Control in Telerobotics," Proceedings of Romansy ' 88 , Udine, Italy.

Bejczy, A.K. and Z. Szakaly. 1987. "Universal Computer Control System for Space Telerobotics," Proceedings of the IEEE Conference on Robotics and Automation, vol. 1, pp. 318-324, Raleigh, NC.

Draper, J.V., W.E. Moore, and J.N. Herndon. 1987. "Effects of Force Reflection on Servomanipulator Task Performance," Proceedings of USDOE/FRG Specialists' Meeting on Remote Systems Technology, Oak Ridge, TN.

Fiorini, P. 1988. "A Versatile Hand for Manipulators," IEEE Control Systems Magazine, vol. 8, no. 5 , pp. 20-24.

Flatau, G., J. Vertut, J.P. Guilbaud, J.C. Germond, and C. Glachet. 1972. "MA22: A Bilateral Servo Master-Slave Manipulator," Proc. 20th Conf. on Remote Systems Technology, pp. 296302.

Goertz, R.C. and W.M. Thompson. 1954. "Electronically Controlled Manipulator," Nucleonics, pp. 46-47.

Goertz, R.C. 1964. "Manipulator Systems Development at ANL," Proceedings of the 12th Conference on Remote Systems Technology, ANS, pp. 117-136.

Hannaford, B. 1987. "Task Level Testing of the JPL-OMV Smart End Effector," Proceedings of the JPL - NASA Workshop on Space Telerobotics, JPL Publication 87-13, vol. 2, pp. 371-380, Pasadena, CA.

Hill, J. 1979. "Study of Modeling and Evaluation of Remote Manipulation Tasks with Force Feedback," Final Report 5, 6 JPL Contract 95-5718, Stanford Research Institute, Menlo Park, CA.

Hill, J.W. and J.K. Salisbury. 1977. "Study to Design and Develop Remote Manipulator Systems," Annual Report, SRI International, Menlo Park, CA.

Kugath, D.A. 1972. Experiments Evaluating Compliance and Force Feedback Effect on Manipulator Performance, General Electric Corporation, NASA-CR-128605, Philadelphia, PA. 
McGovern, D.E. 1974. "Factors Affecting Control Allocation for Augmented Remote Manipulation," Ph.D. Dissertation, Design Division, Department of Mechanical Engineering Stanford University, CA.

Pepper, R.L. and P.K. Kaomea. 1988. "Teleoperation: Telepresence and Performance Assessment," Proc. Int. Symposium Teleoperation and Control, pp. 227-234, Ergonomics Society.

Raibert, M.H. and J.J. Craig. 1981. "Hybrid Position/Force Control of Manipulators," ASME J. of Dynamic Systems, Measurement, and Control, vol. 102, pp. 126-133.

Szakalay, Z., W.S. Kim, and A.K. Bejczy. 1989. "Force-Reflecting Teleoperated System with Shared and Compliant Control Capabilities," Proceedings NASA Conference on Space Telerobotics, JPL Publication 89-7, Pasadena, CA.

Vertut, J. 1976. "Experience and Remarks on Manipulator Evaluation," in Performance Evaluation of Programmable Robots and Manipulators, ed. T.B. Sheridan, National Bureau of Standards Special Publication 459.

Whitney, D.E. 1985. "Historical Perspectives and State of the Art in Robot Force Control," Proceedings of IEEE Conference on Robotics and Automation, pp. 262-268. 
Appendix A

Modular Task Board For Telemanipulation Experiments

Douglas McAffee 


\section{MODULAR TASK BOARD FOR ROBOTICS AND TELEOPERATORS}

The Modular Task Board, depicted in the following pages, was developed for testing human subjects in experiments using the Advanced Teleoperator System.

The Modular Task Board is composed of as many as nine separate "task windows" designed to simulate generic and actual tasks that a teleoperator/telerobotic system may be called upon to perform.

\section{Main Features}

Task Layout Easily Rearranged

The distances between tasks can be changed. Sometimes it is desirable to vary the relative location and inter-task distances to offset human learning, check adaptation, or provide variety. Also, inter-task distances can be easily changed if after use modification proves desirable.

Accommodates a Variety of Task Sizes

The task board features removable window frames so that tasks wider than the standard 7-in. by 7 -in. window can be accommodated. The task frame itself is modular and allows for larger tasks (up to $7 \mathrm{in}$. by $21 \mathrm{in}$.) to be mounted flush to the task board surface by removing intermediary frame members.

Task Modification Easy and Inexpensive

New tasks can be easily fabricated and added without removing or remaking the entire task board. If a task proves to be undesirable or becomes obsolete, the whole task board does not have to be scraped.

No Down Time

New tasks, modified tasks, or repairs can be made off-line to task board usage, thereby allowing for continuous system operation. 
Task Isolation

Often multiple task boards consist of a complicated, congested array of multiple tasks, or, to avoid overcrowding, they limit the number of separate tasks that can be performed.

Sometimes it is desirable to test only one or two tasks sequentially and to focus on them only. The Modular Task Board allows the user to isolate individual tasks by filling the balance of the task board surface with "blank task windows."

Even Task Board Profile

The Modular Task Board is designed to allow most tasks to mount almost flush to the task board surface. This helps to avoid complicated task board topologies that could distract from the experiments being performed.

Adjustable Inclination

The task board inclination can be adjusted to any angle between horizontal and vertical.

Optimal Use of the Puma 560 Work Envelope

The task board is designed to fit optimally in the Puma 560's work envelope. When positioned properly, the entire task board surface can be reached. 


\section{MODULAR TASK BOARD}

\section{\$or}

ROBOTICS and TELEOPERATORS

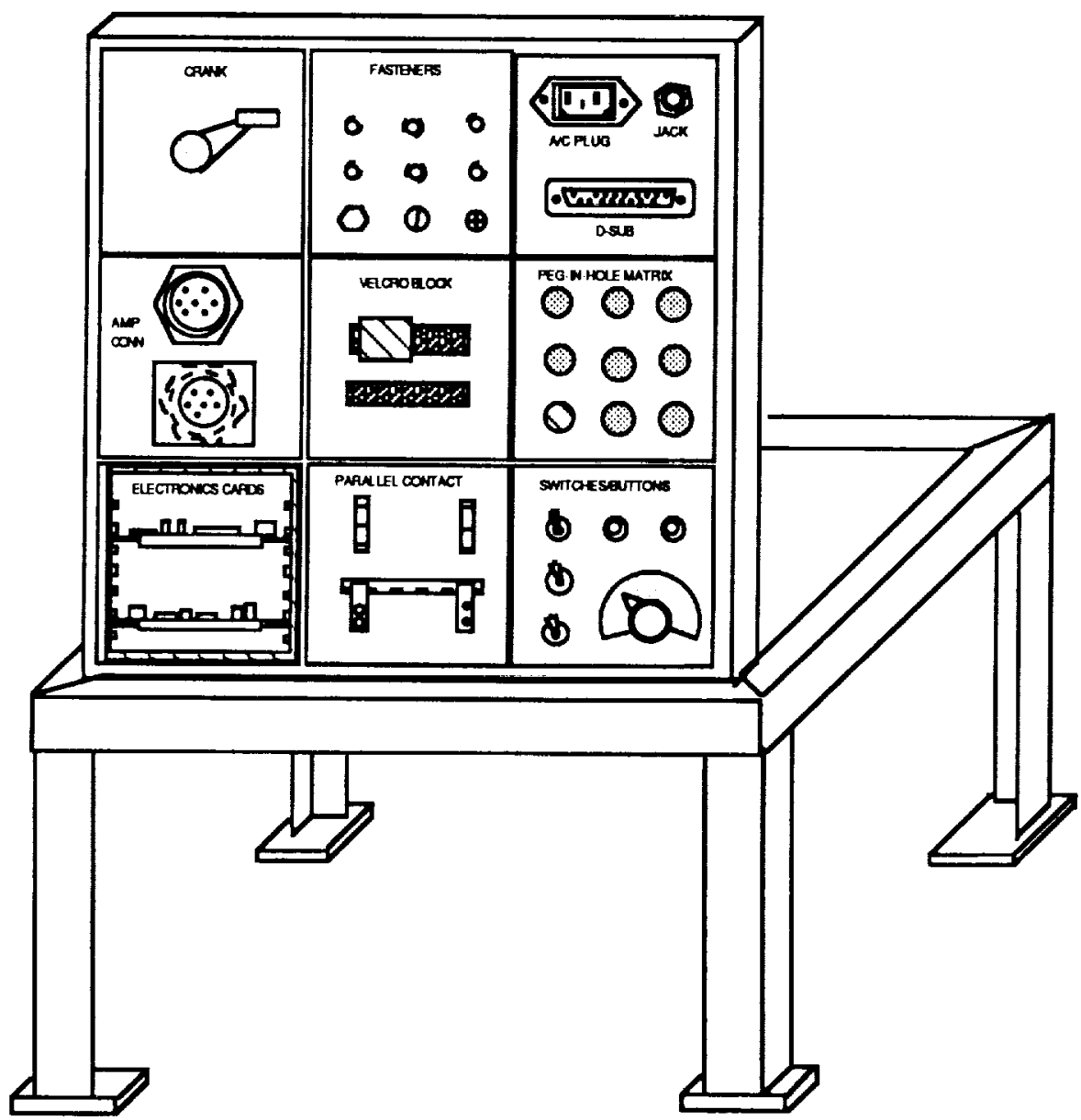




\section{MODULAR TASK BOARD \\ FOR ROBOTICS AND TELEOPERATORS}

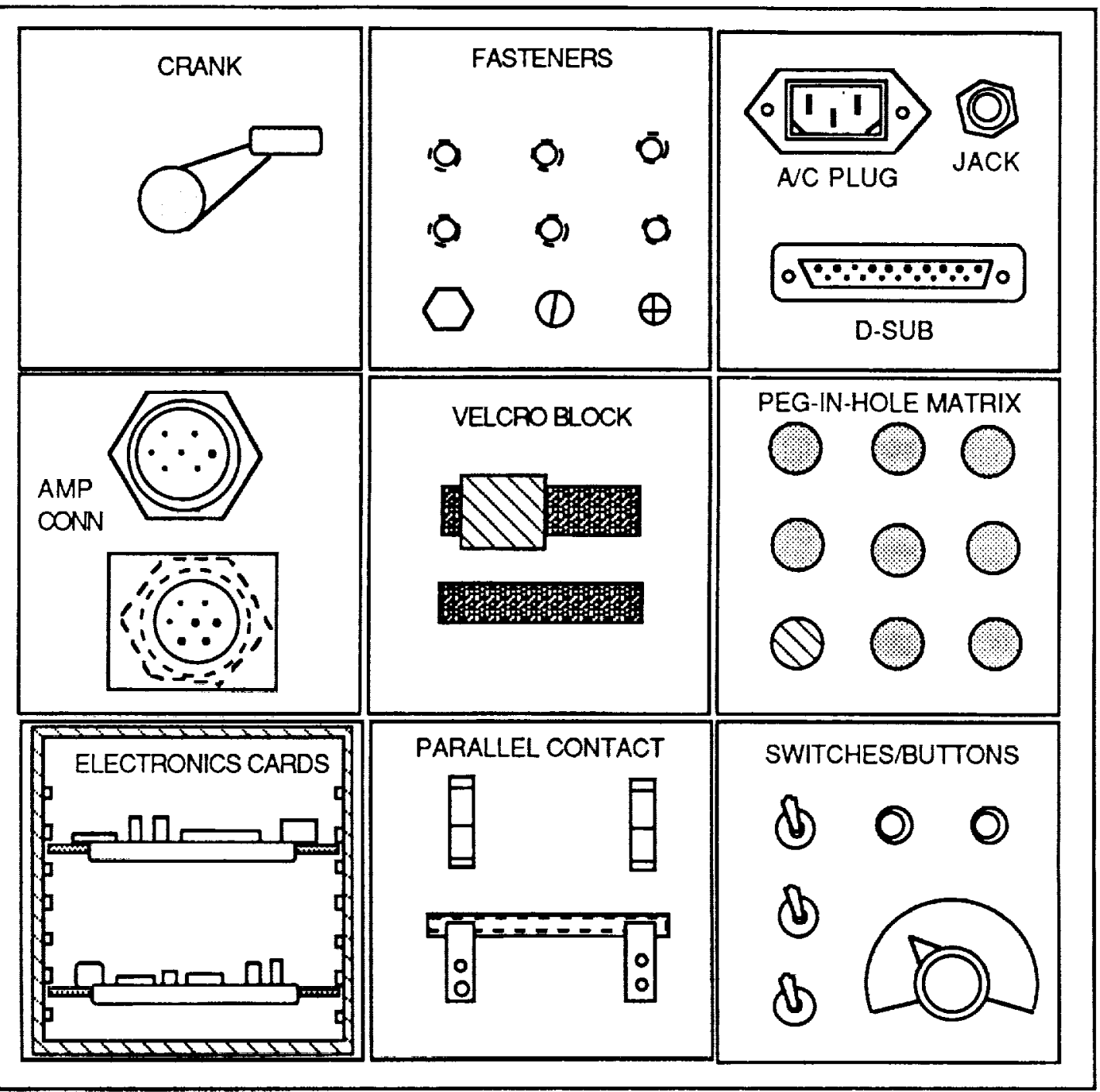


TASK WINDOWS CAN BE REARRANGED

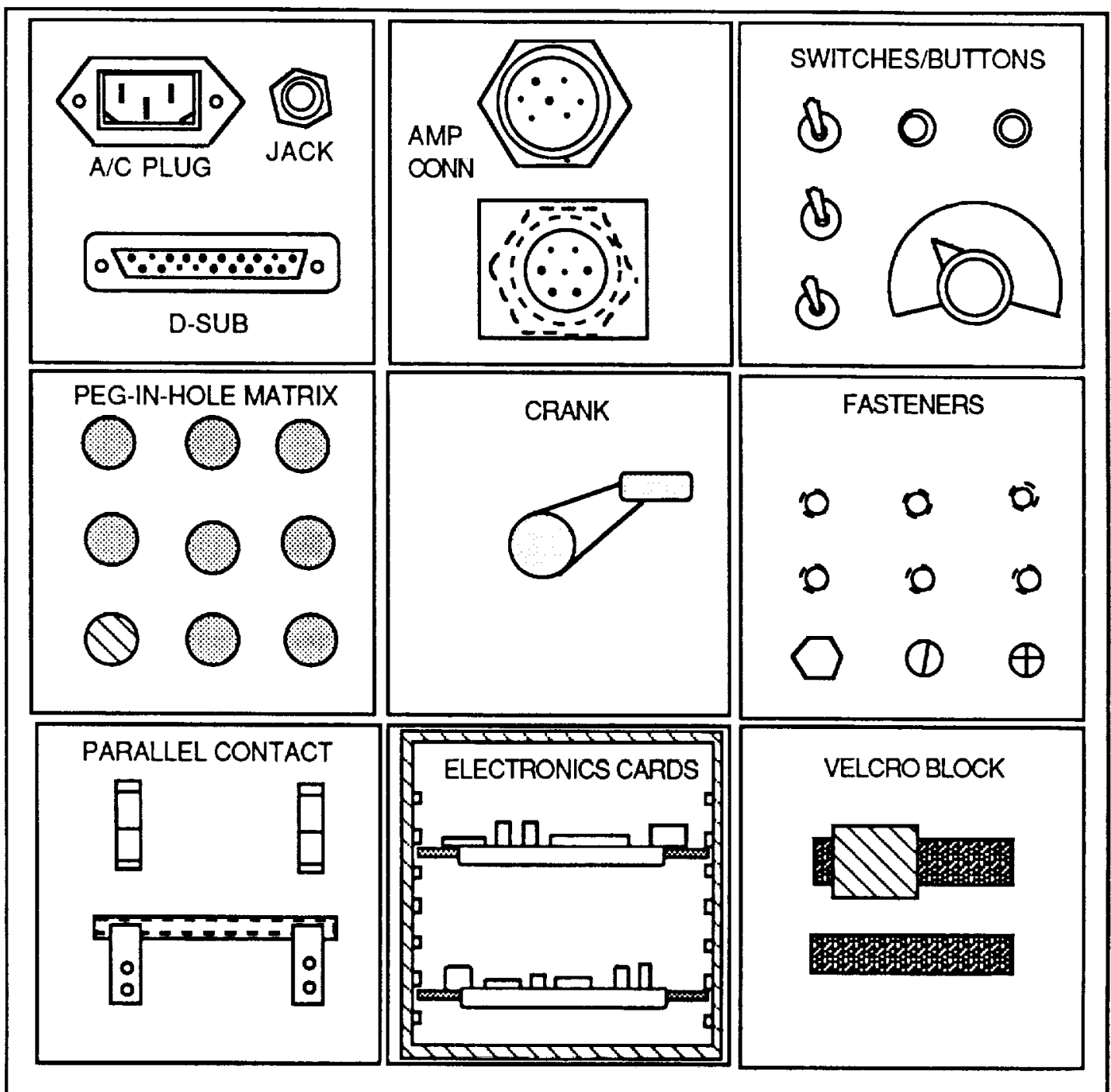


TASKS CAN BE ISOLATED BY USING BLANK WINDOWS

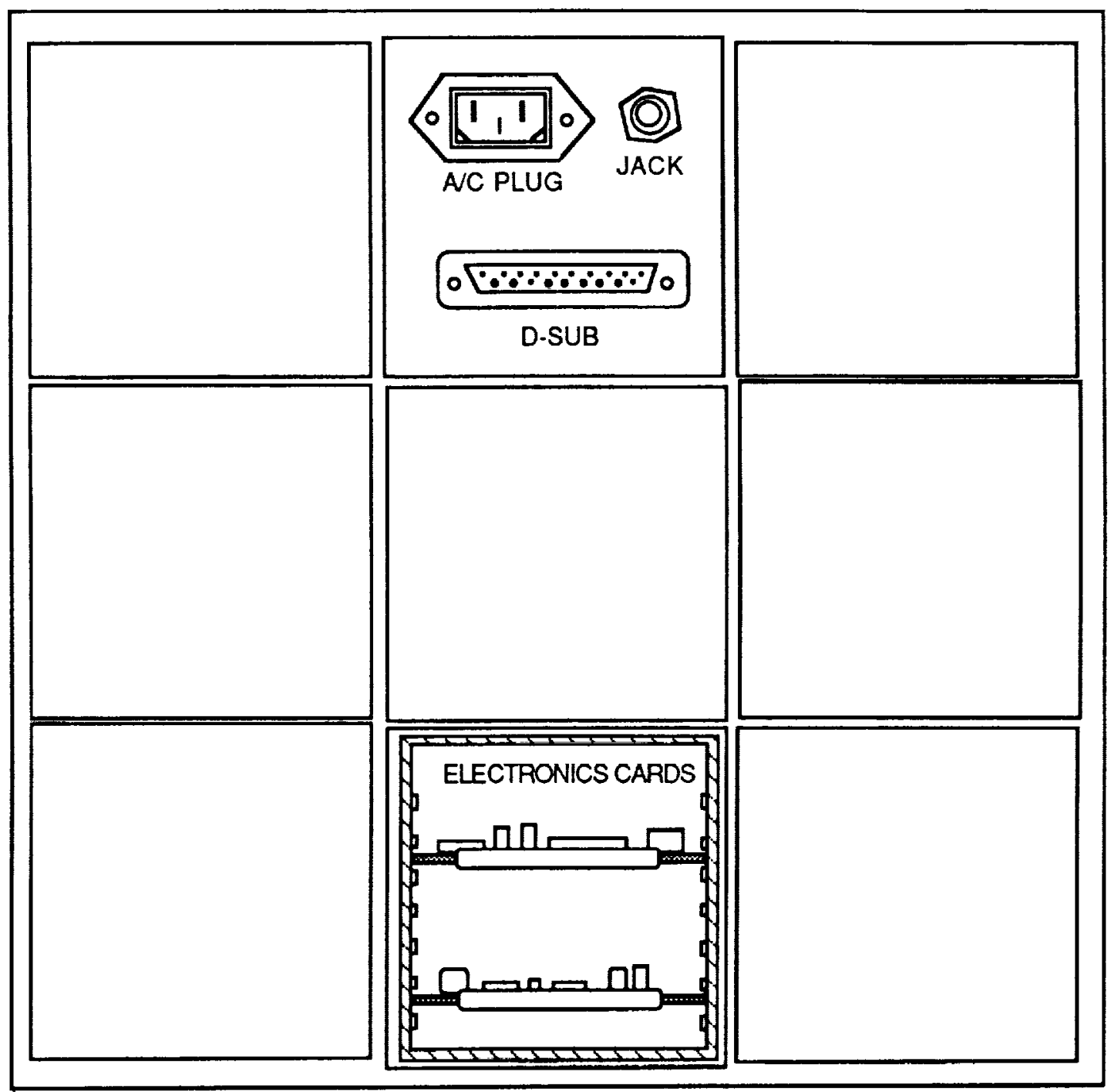


Tasks Can Vary In Sizs

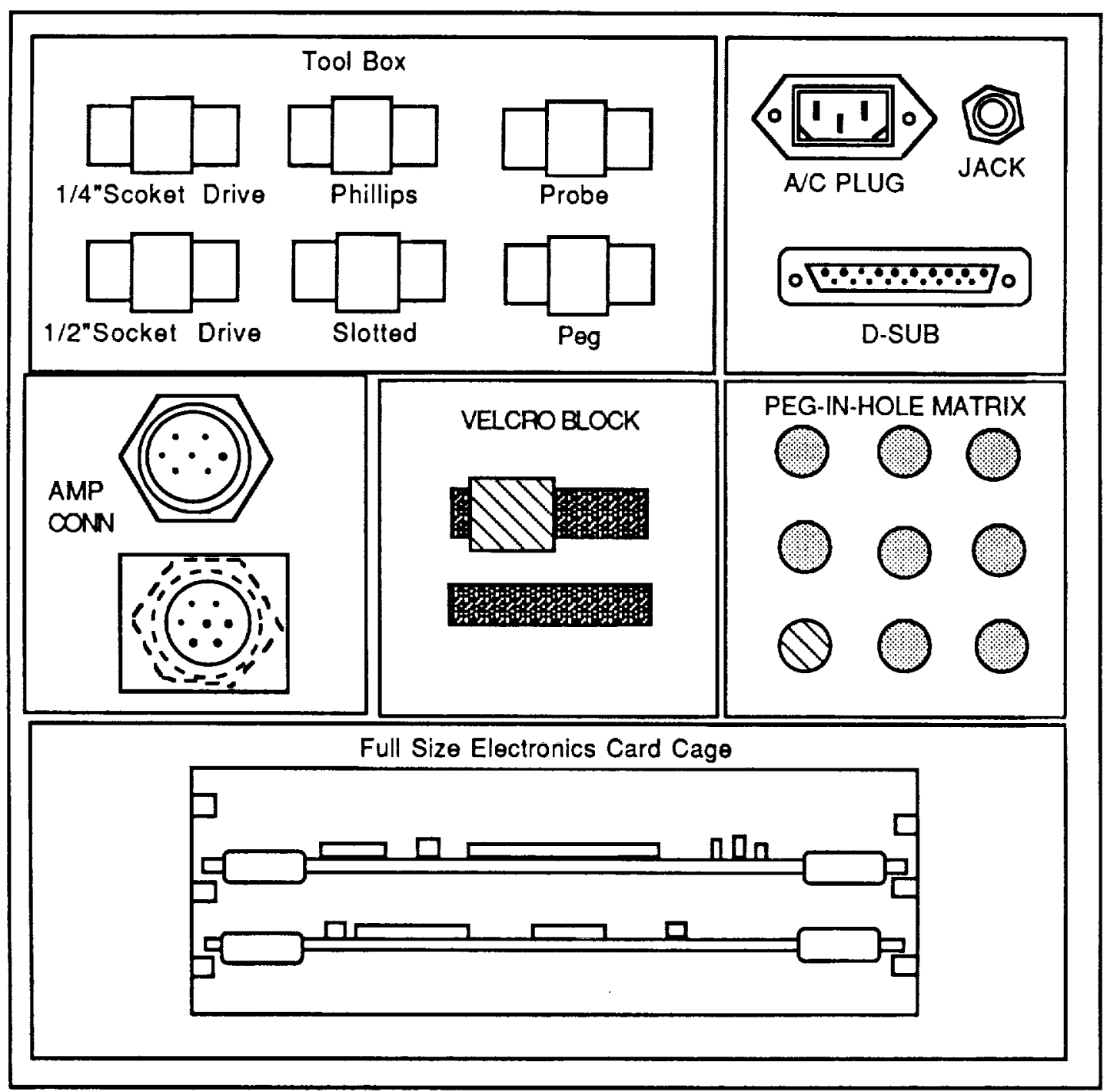




\section{Modular Task Board \\ Mounting Comfigurations}


MODULAR TASK BOARD and PUMA 550 ROBOT ARM
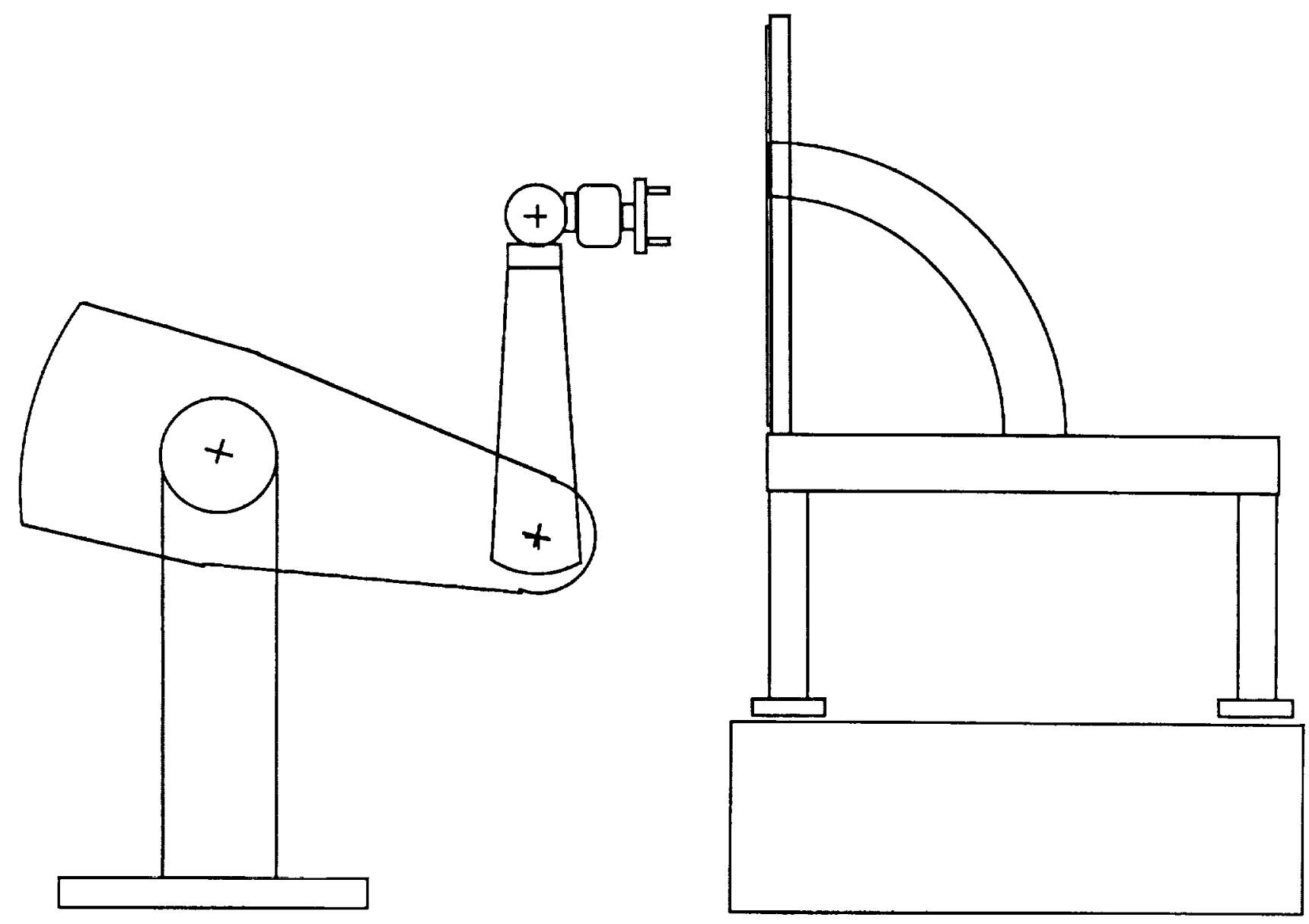


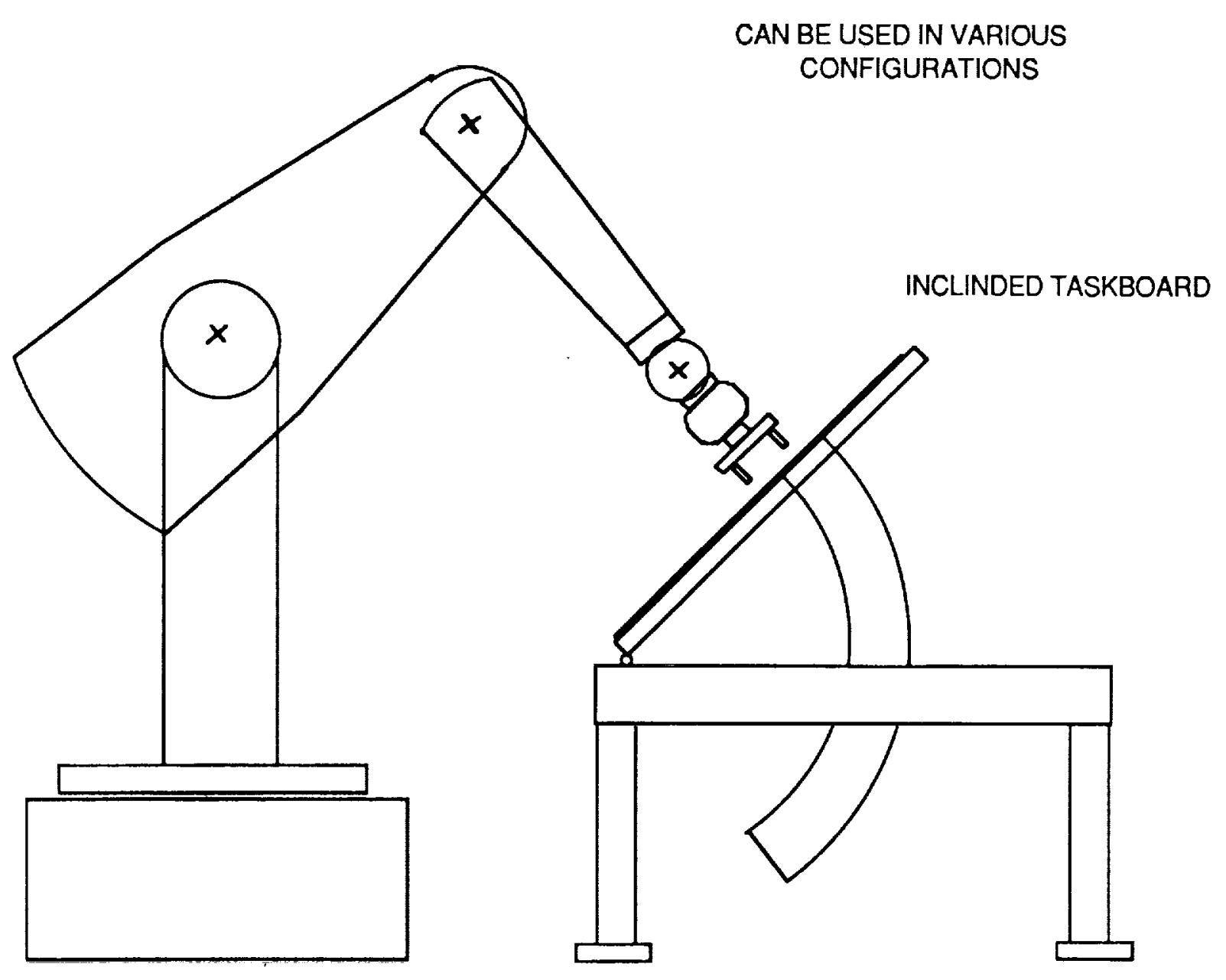




\section{MODULAR TASK BOARD and PUMA 560 ROBOT ARM}

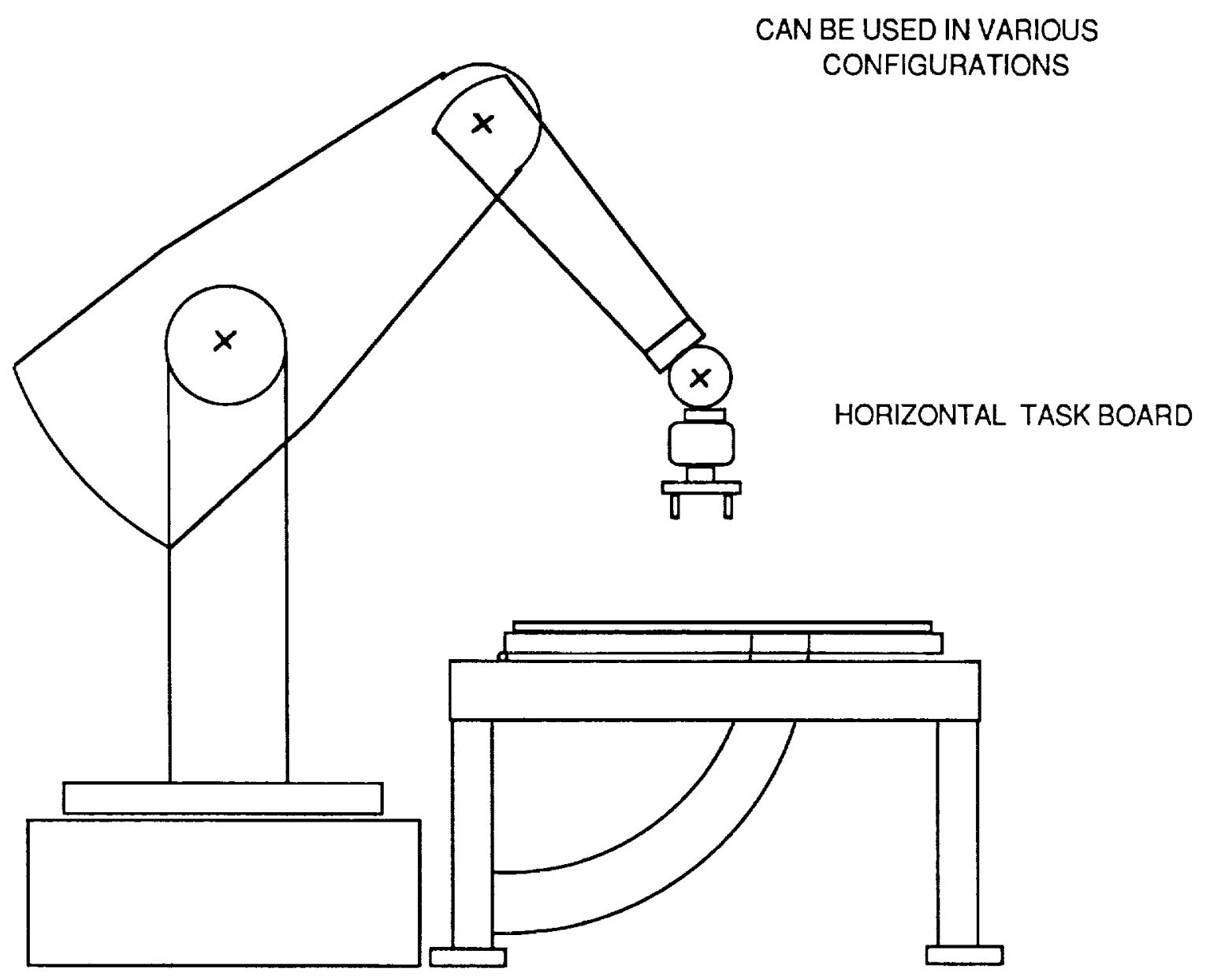


MODULAR TASK BOARD OPTIMIZED FOR USE IN PUMA WORK ENVELOPE

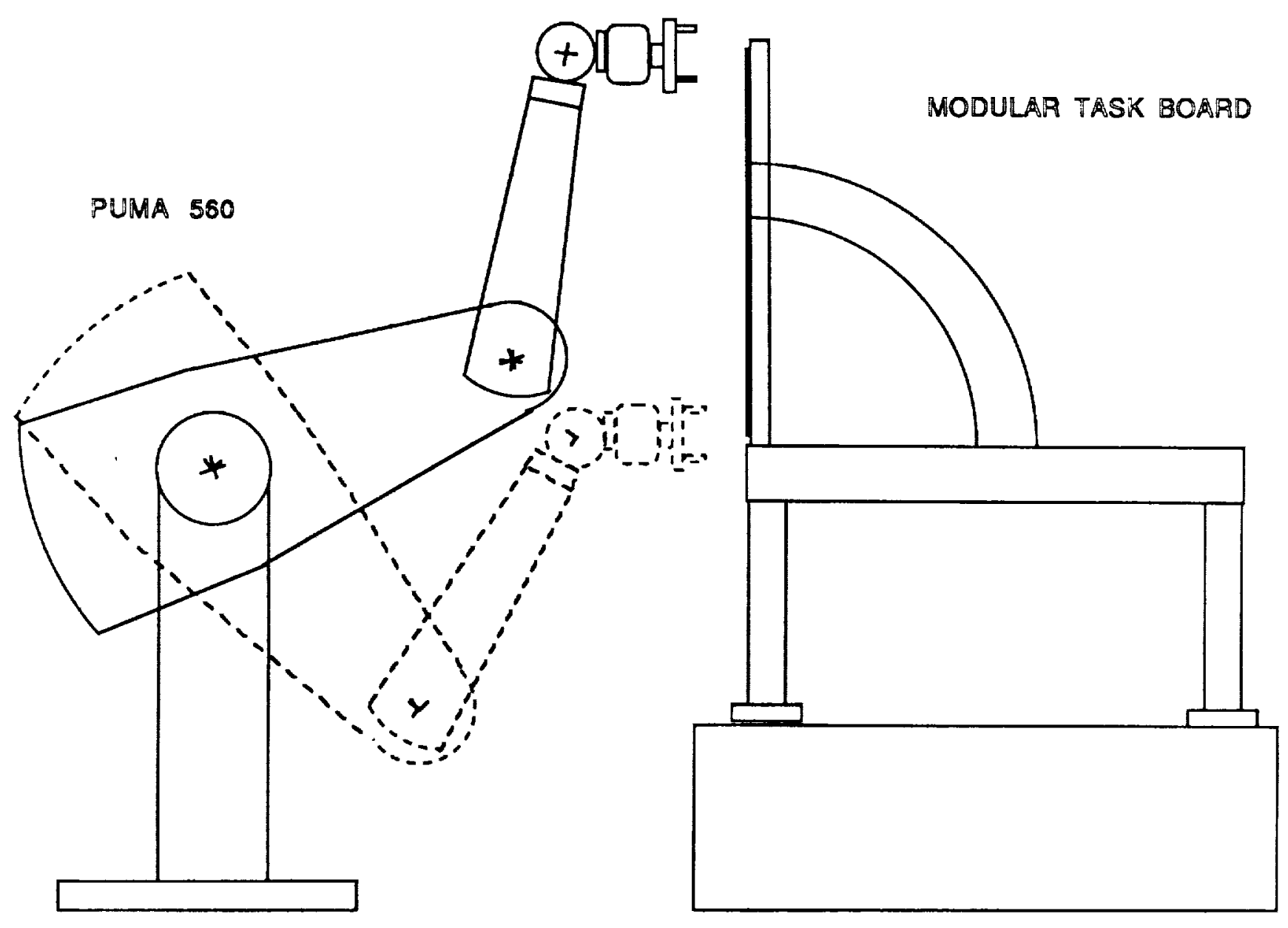


Modular Task Board

Task Wimdow Frame 


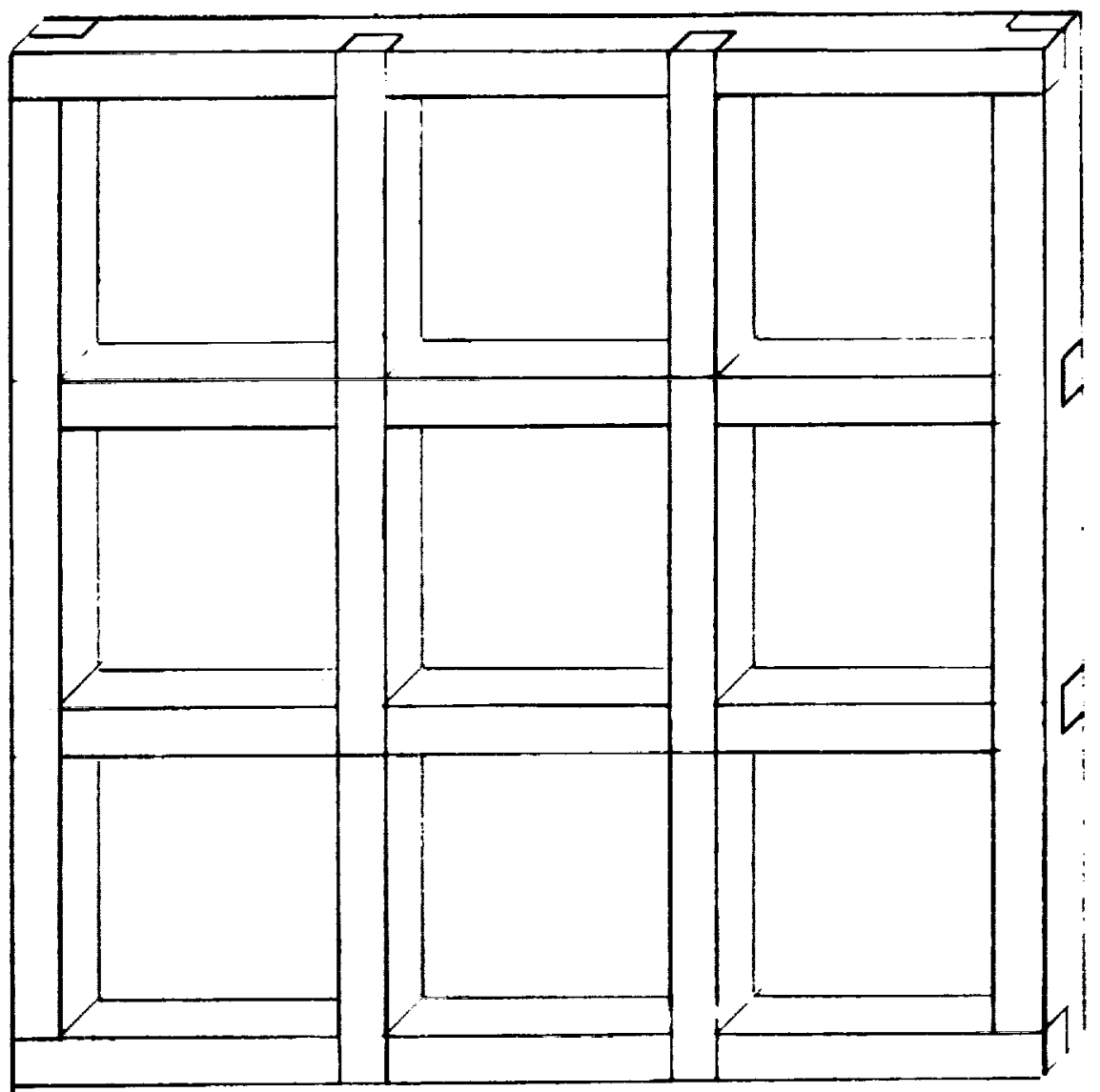




\begin{tabular}{|c|c|c|}
\hline (PREPARED BY) & (DATE) & (REPORT NO.) \\
\hline (CHECKEO BY) & (DATE) & (PROJECT) \\
\hline
\end{tabular}

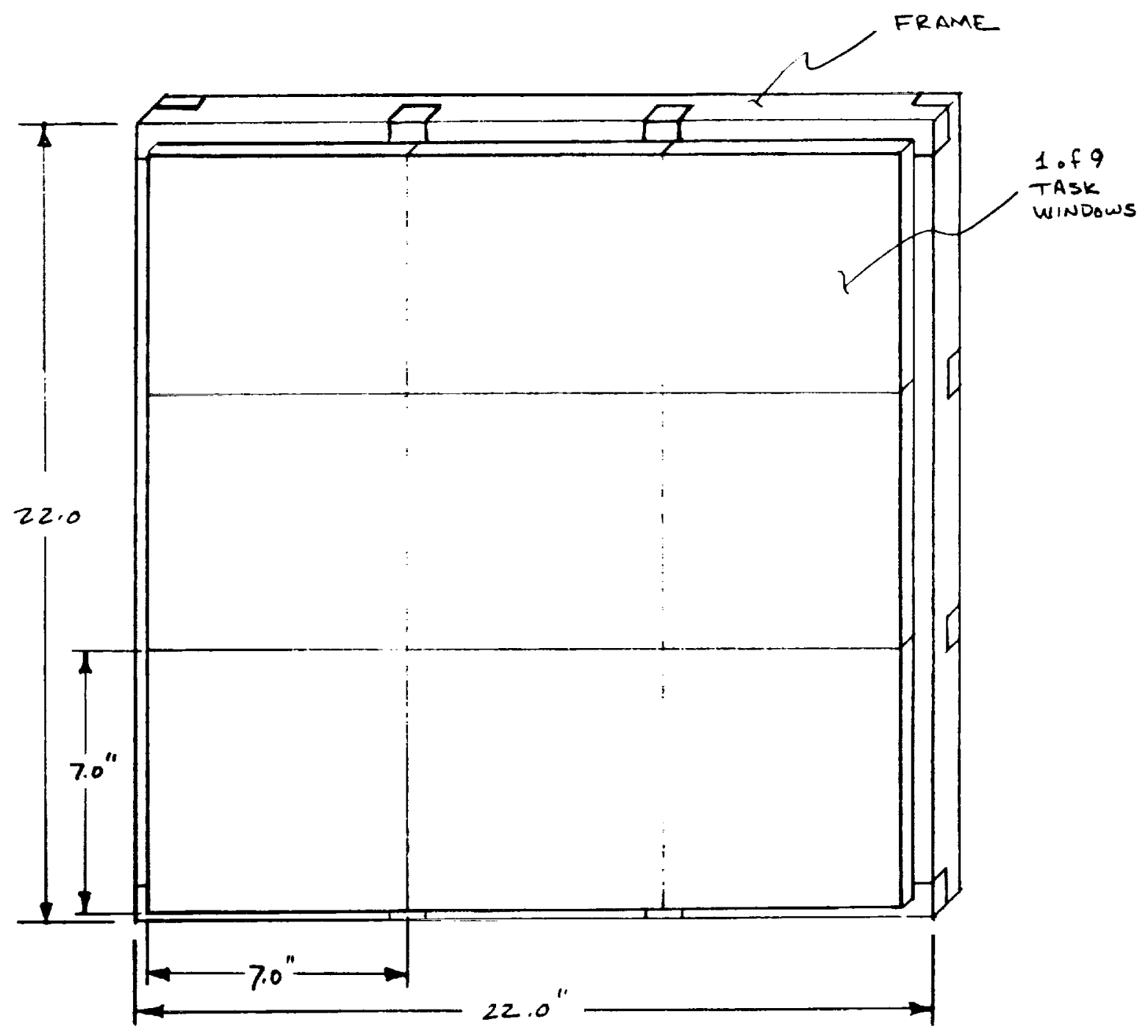


JP L

PAGE

OF

(PREPARED BY)

(DATE)

(CHECKED BY)

(DATE)

(PROJECT)

TITLE EXPLODED PARTS VIEW OF FRAMAF

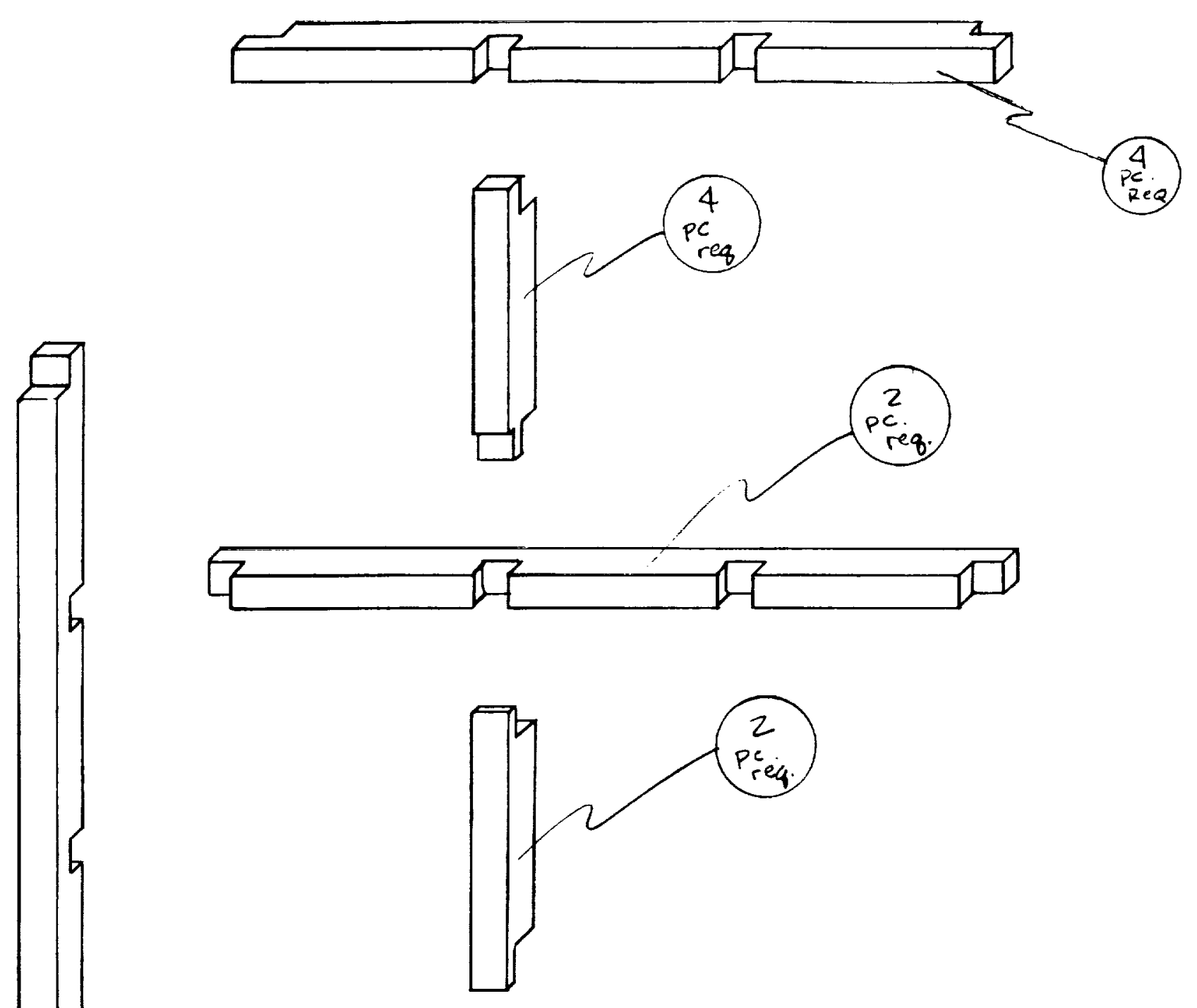

80 
JP

PAGE 1 OF

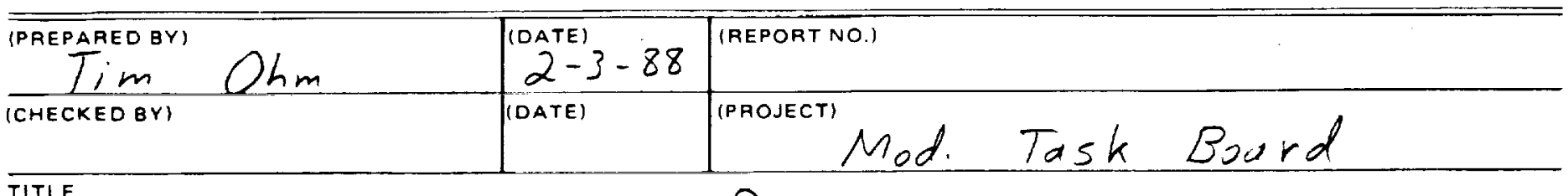

TITLE

Task BoArd Frame Piece
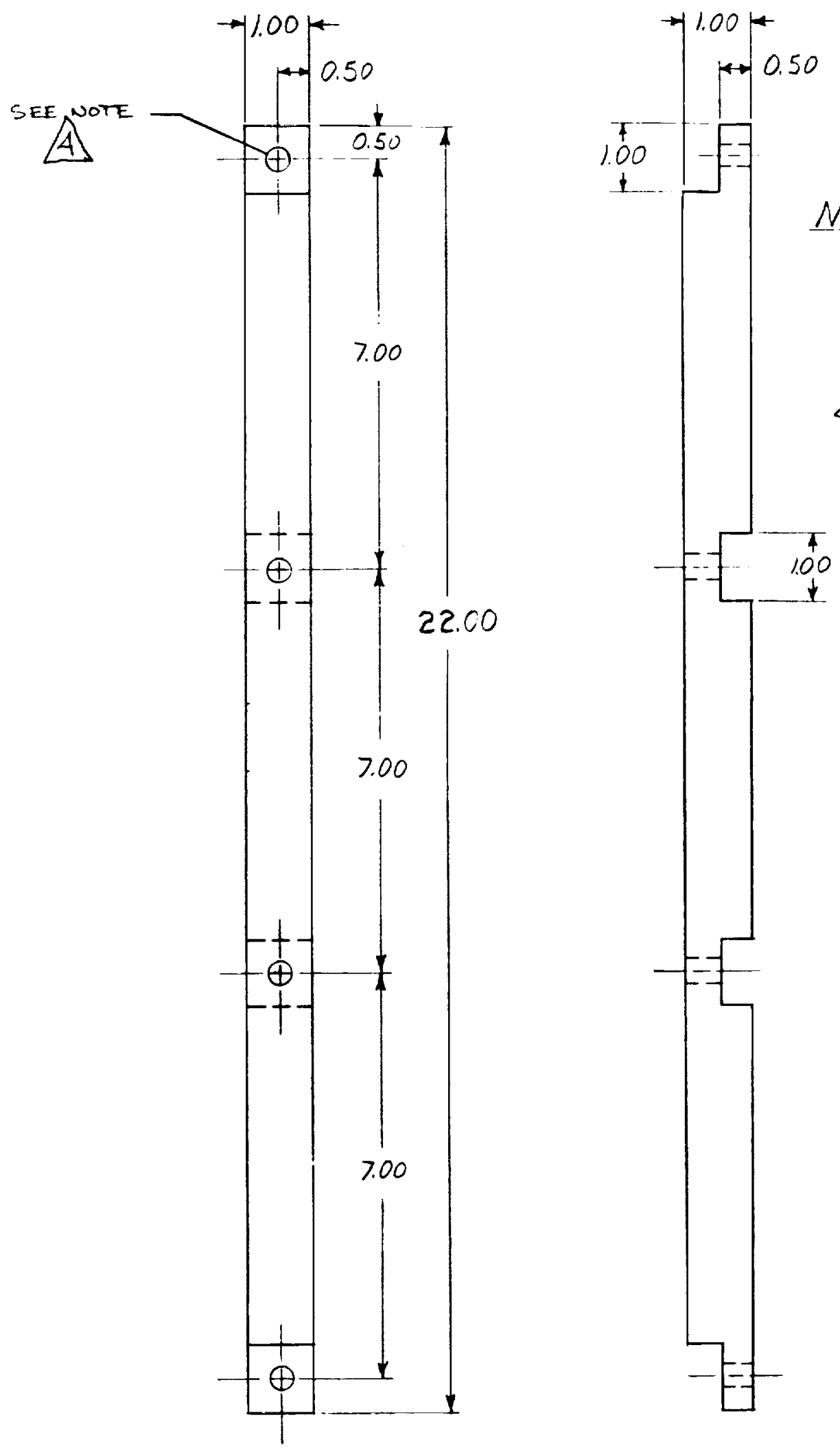

NOTES:

1. Al 6061

2. 2 Reid of ea vier.

3. DIM. ARE IN.

4. 2 VERSIONS $A \& B$ YER A: TAP HOLS S AT BOTH ENDS $Y_{1}-20$, AND THE TWO CENTRAL HOLES 1/4-20 CLEARANCE VERB TAP TWO CENTRAL HOLES Y/4.20, AND DRIL THE Has AT BOTH TALE $1 / 4-20$ CLEAKANXL.

81 
NFL

PAGE 2 OF 4

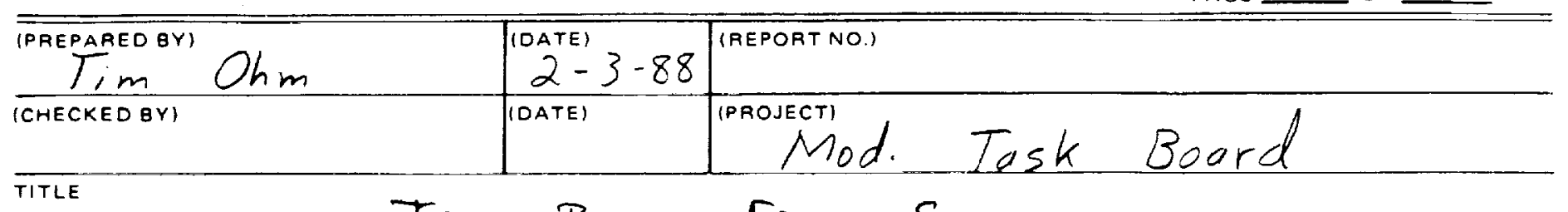

TitLe Task Board Frame Support
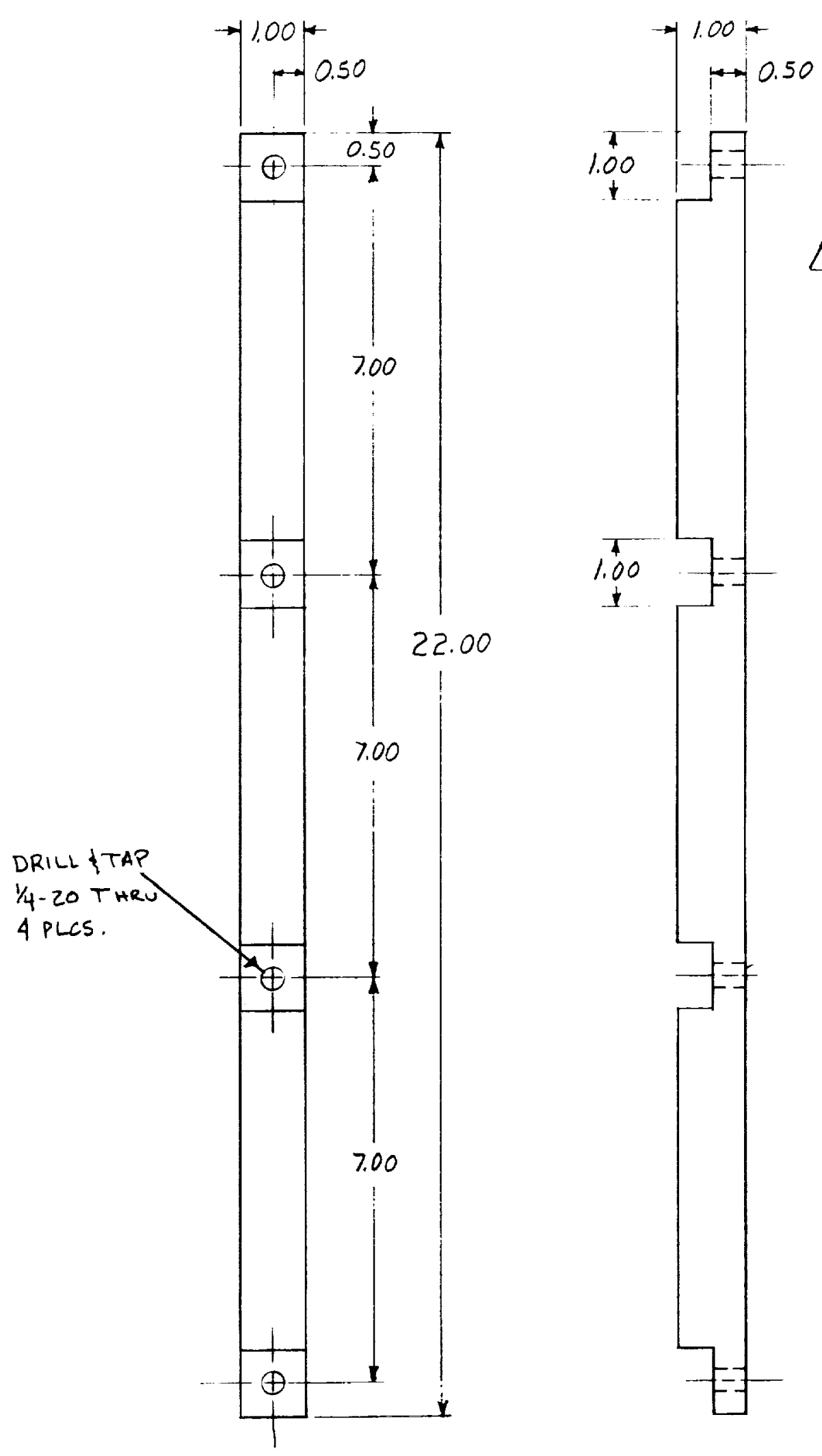

NOTES:

1. A1 6061

2. 2 Regis

3. DIM. ARE IN.

82 
PL

PAGE 3 OF 4

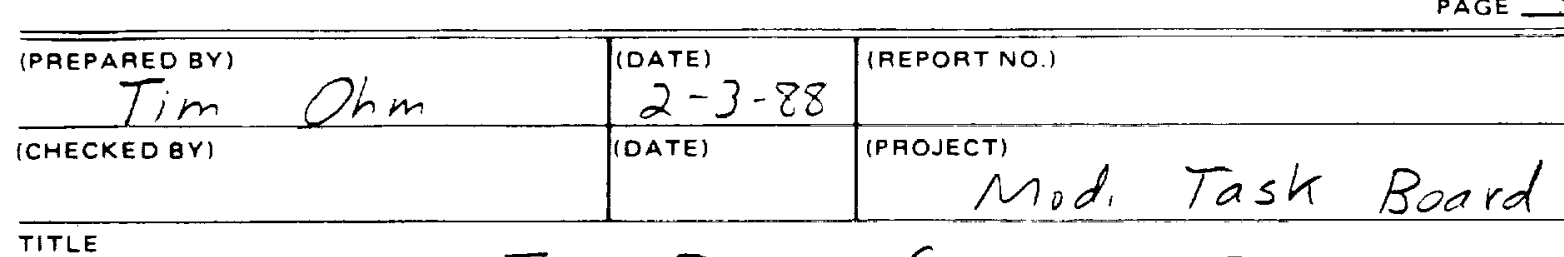

TitLe Task Board Connecting Strut
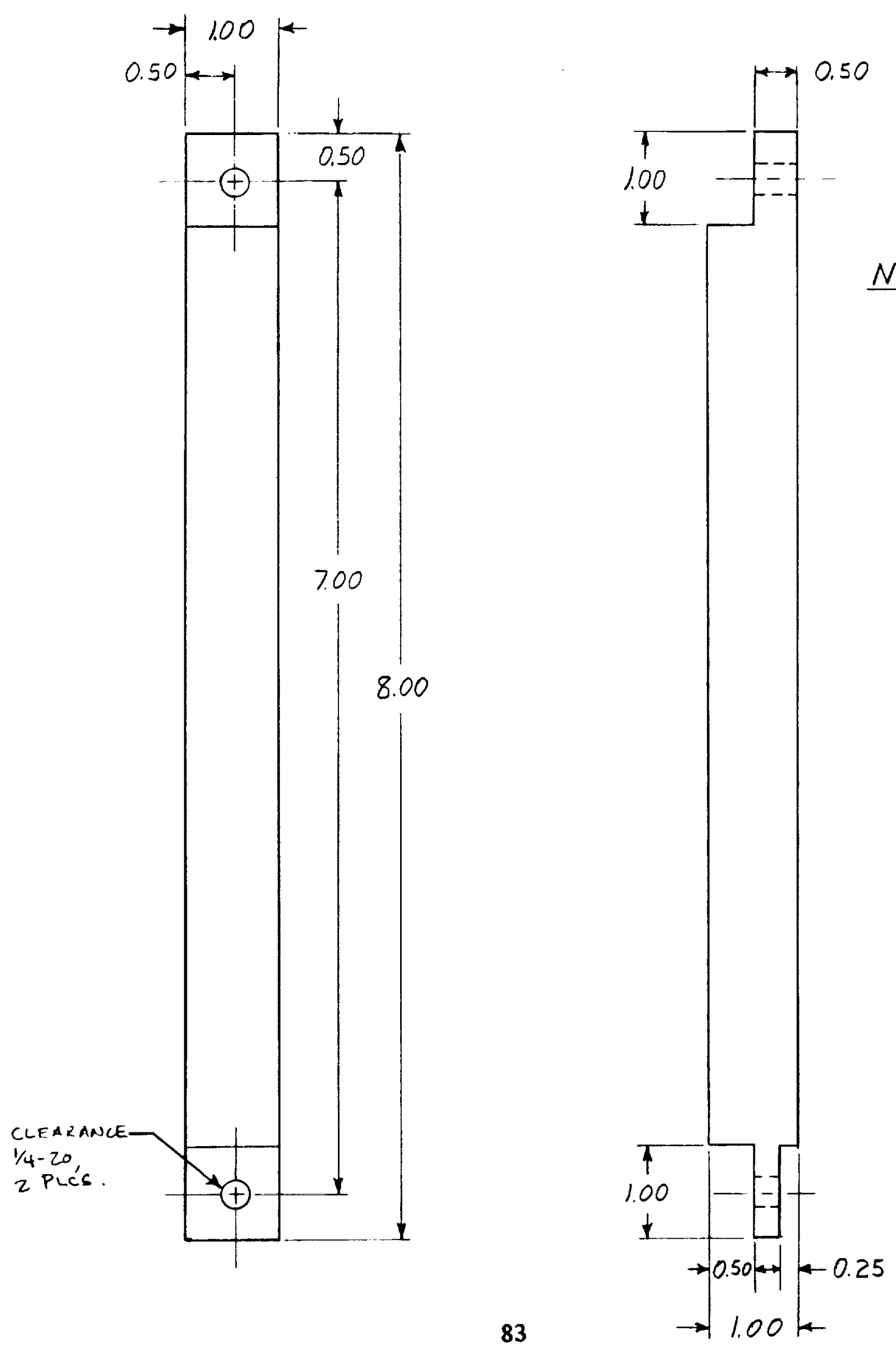

83

NOTES:

1. Al 6061

2. 4 Reg'd

3. DIM ARE IN. 
JP

PAGE 4 OF 4

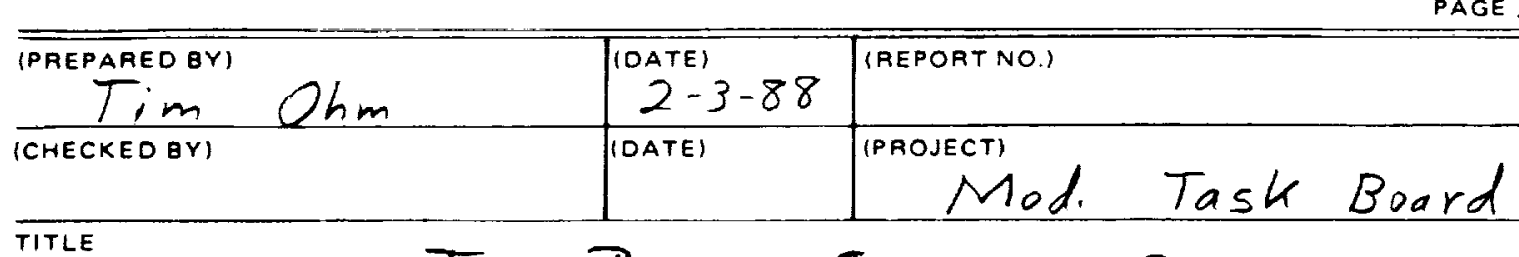

title Task Board Separating Strut
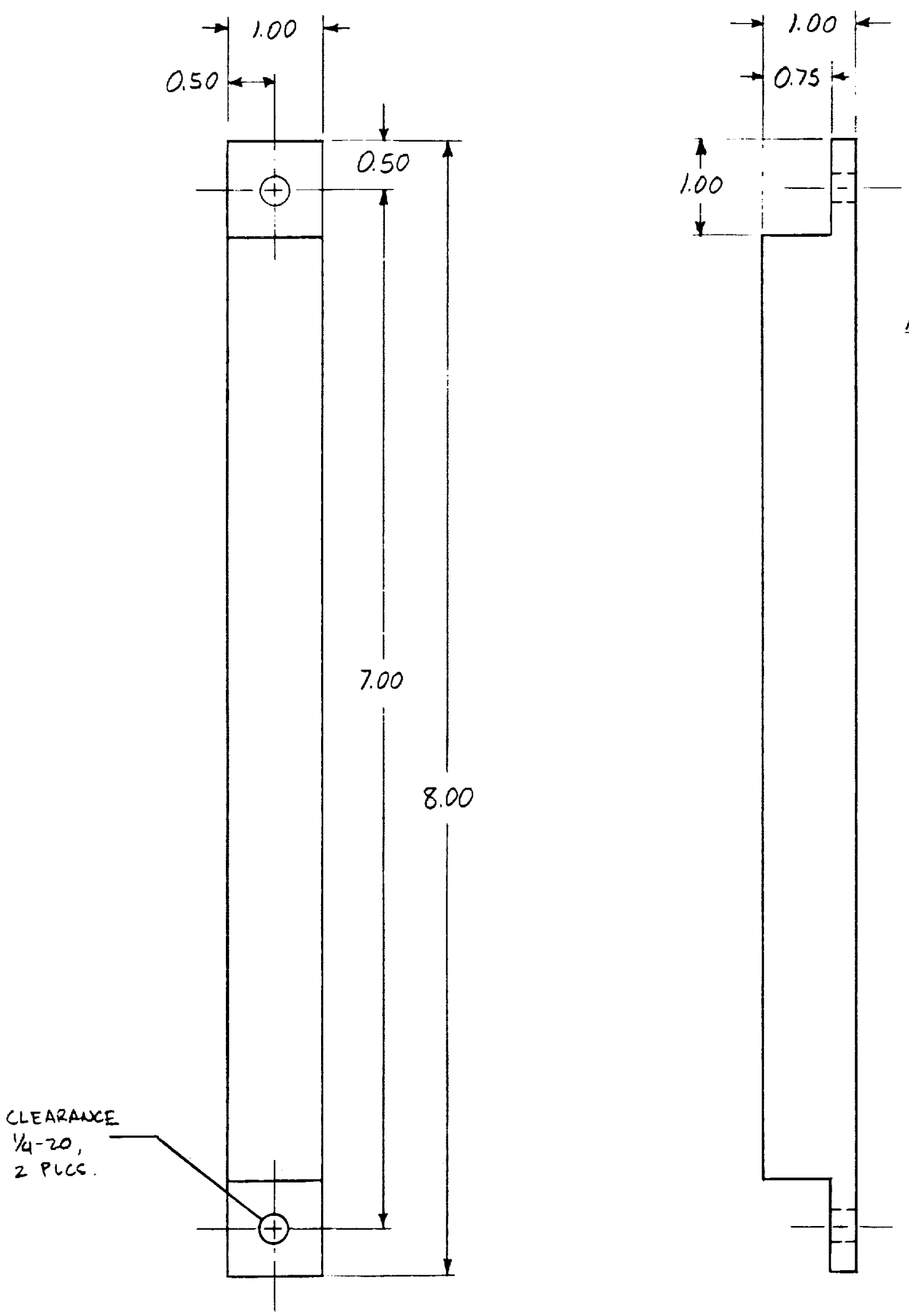

NOTES:

1. Al 6061

2. 2 Reg'd

3. DIM. ARE IN.

84 


\section{Modular Task Board \\ Task Windows}




\begin{tabular}{|c|c|c|}
\hline $\begin{array}{l}\text { (PREPARED } \\
\text { Brent Gy }\end{array}$ & $\begin{array}{l}\text { (DATE) } \\
9-6-88\end{array}$ & (REPORT NO.) \\
\hline (CHECKEDEY) JJ & (DATE) & (PROJECT) \\
\hline
\end{tabular}

TITLE

PEG IN HOLE TASK

WINDOW I OFI

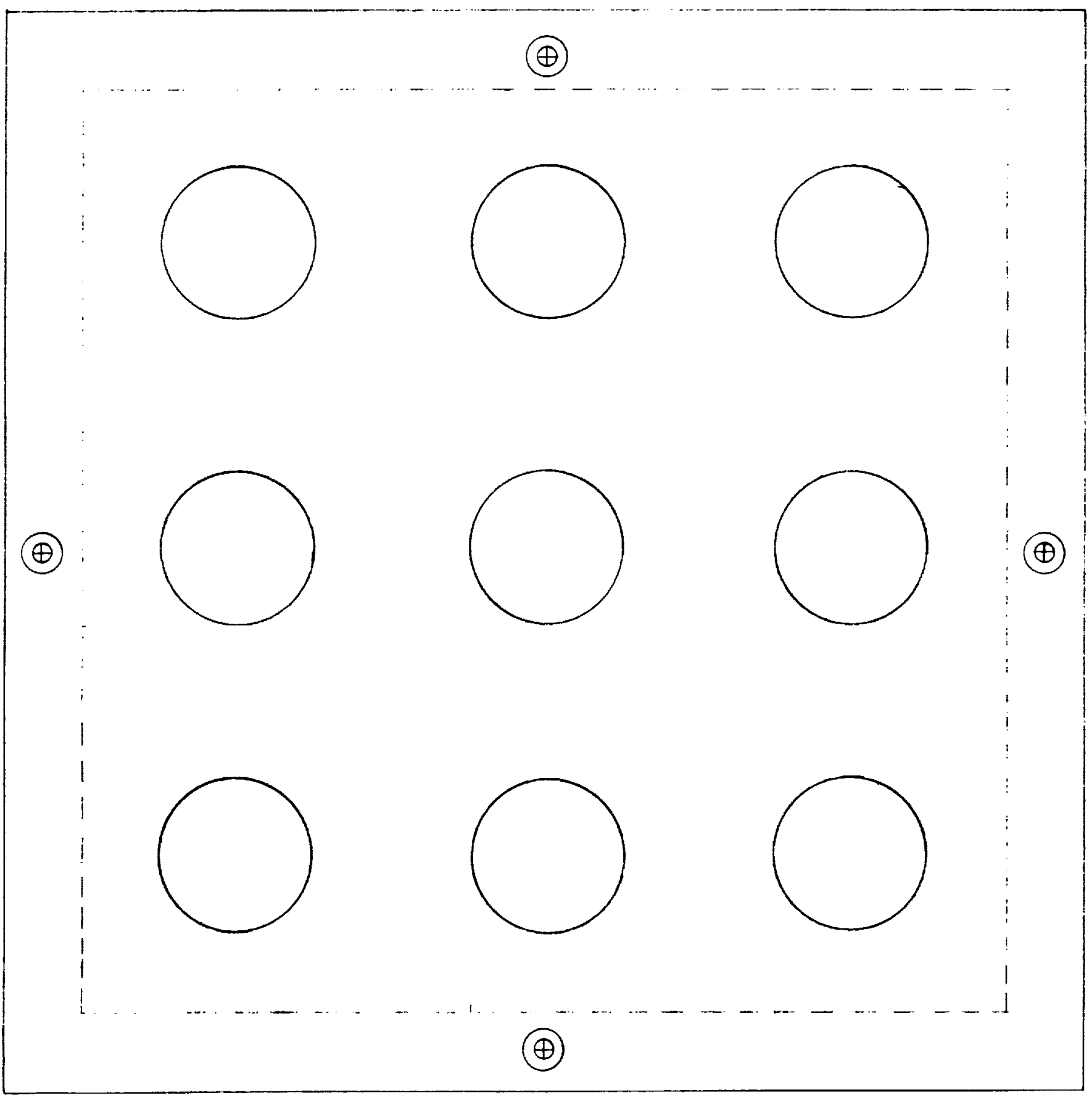

NOTE: SCALE $1: 1$ 
L

PAGE

OF

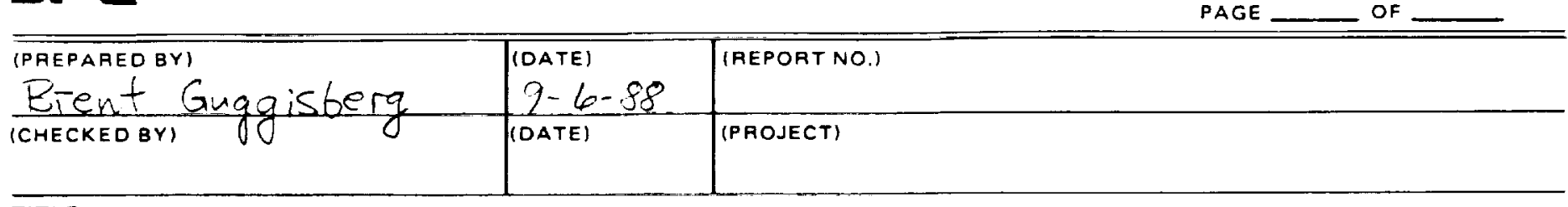

TITLE

PES N HOLE TASK

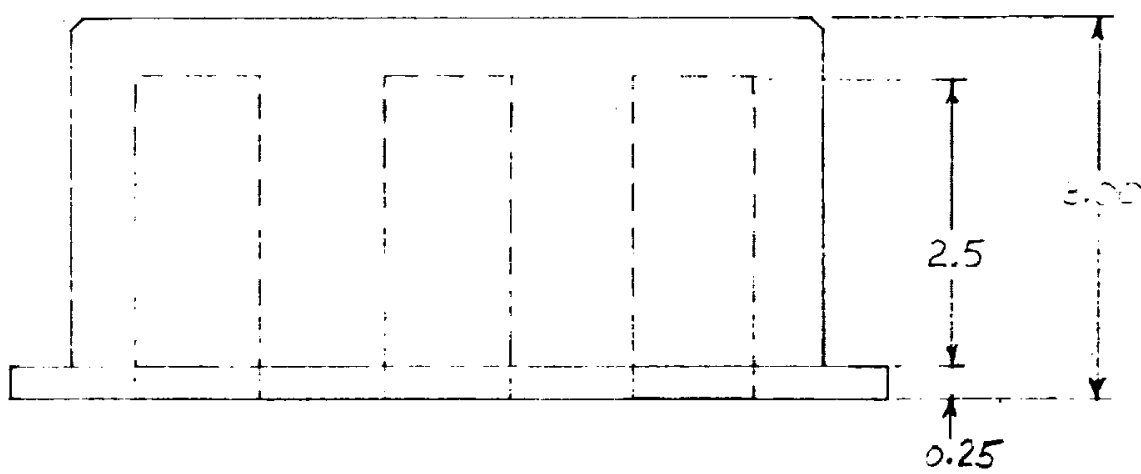

Window I of?

NOTES:

1. SCALE 1:2

2. DIM ARE IN.

3. Al 6041 BLOCK

* 4. 304 STAINLESS STEel
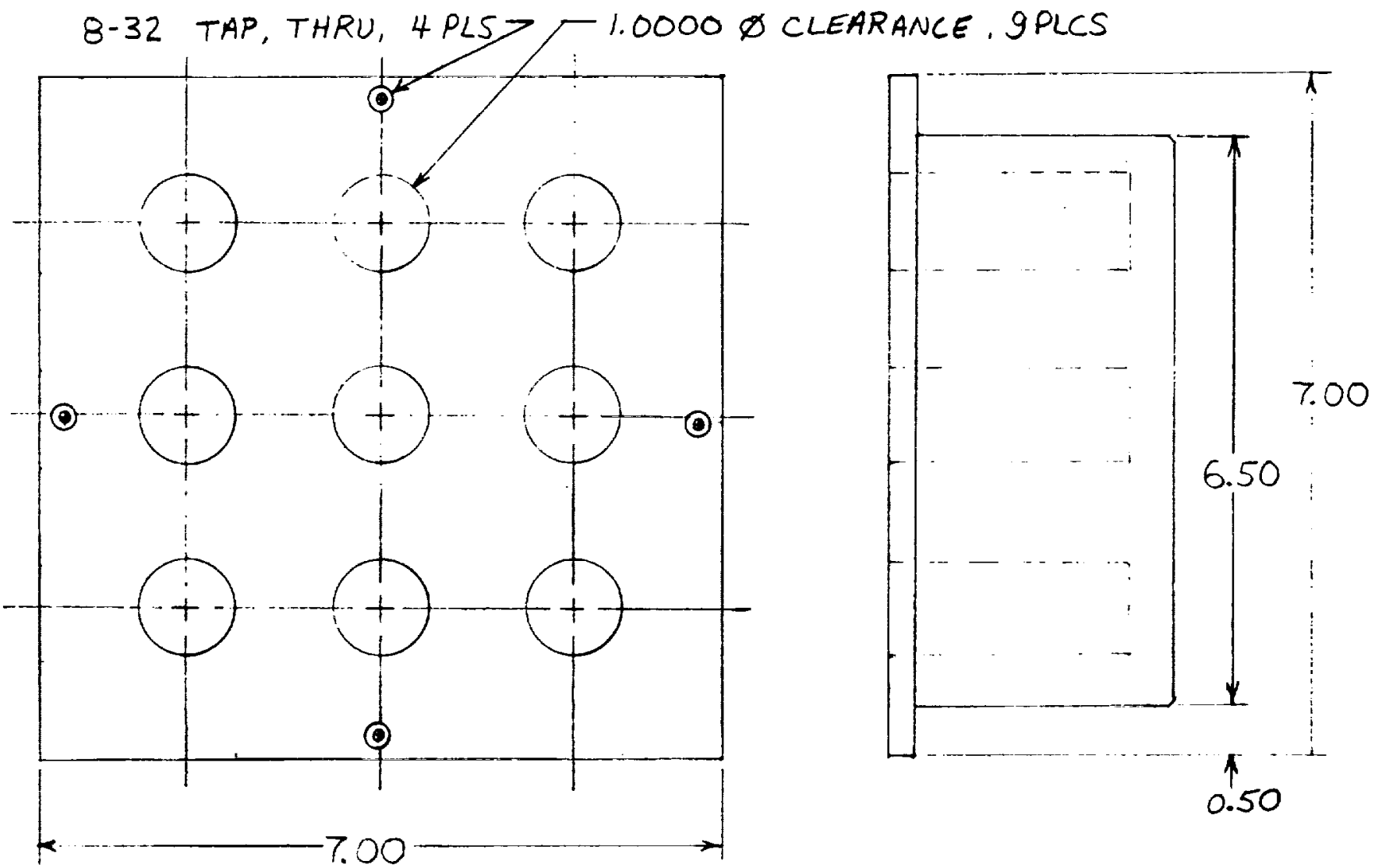

ORIGINAL PAGE IS OF POOR QUALIa

87 


\begin{tabular}{c|c}
\hline (PREPAREO & (DATE) \\
Brent Gujgisbery & $9-6-88$ \\
\hline (CHECKEDBY & (DATE)
\end{tabular}

MATING AND UNMATING OF THREE STANDARD ELECTRICAL CONNELTORS

(๑)

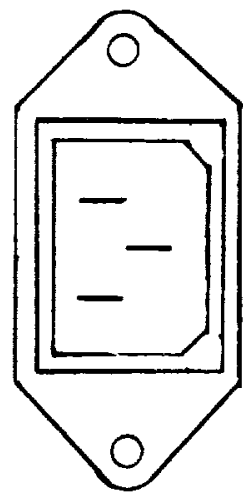

RECEPT CONNECTOR RECESSEO, 3 PIN FOR HP EQUIPMENT

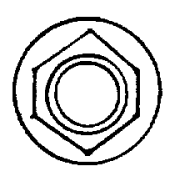

RECEPT CONNECTOR MICROPHONE

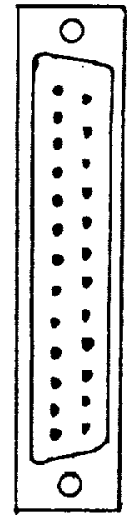

RECEPT CONNECTOR ITT CANON, RECTAGULAR 


\begin{tabular}{l|l}
\hline (PAEPARED BY) & (DATE) \\
Brent Guggister) & $9-6-88$ \\
\hline (CHECKEDBY) & (DATE)
\end{tabular}

(๑)

(๑)
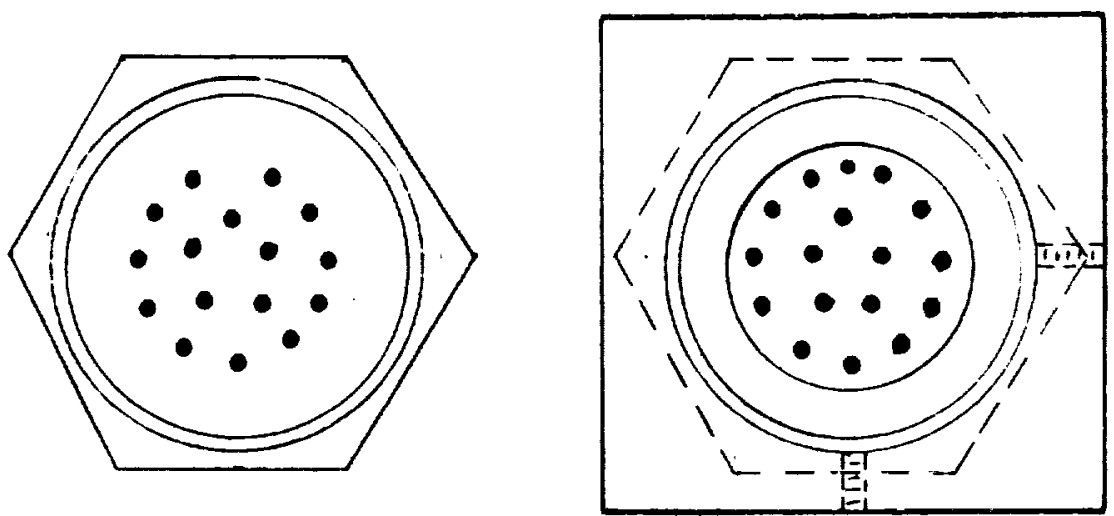

\section{BENDIX CONNECTOR \\ RECEPT}

NOTE: IN ORDER FOR THE GRIPPER FINGER TO MATE, LOCK, UNLOCK, AND UNMATE THE BENDIX STYLE CONNECTOR ABOVE, A RECTANGULAR ADAPTOR WAS FABRICATED FOR THE CIRCULAR PLUG. 
JP

PAGE

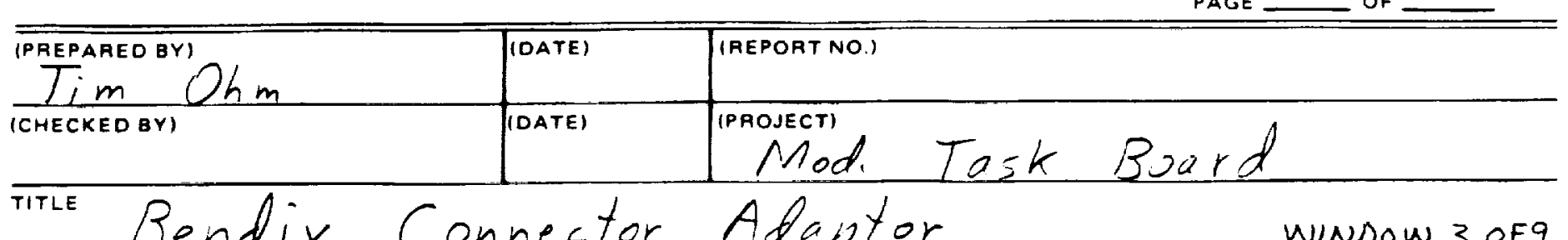

True Bendix Connector Adaptor

WINDOW 3 OF 9

Notes:

1. Al 6061
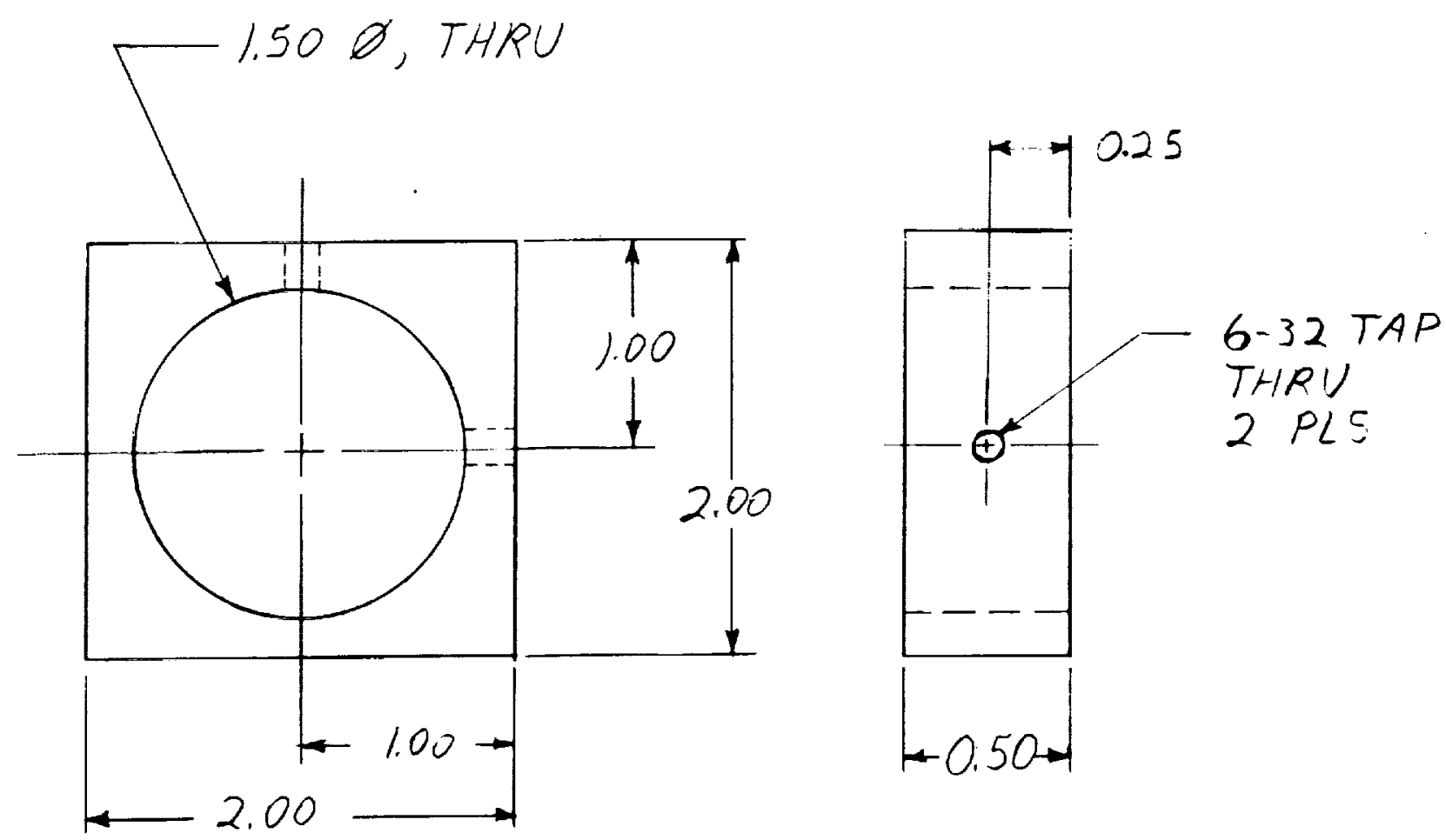

90 


\begin{tabular}{l|l|}
\hline (PREPAAEO BY) & (DATE) \\
Brent Guggisberg & $7-6-88$ \\
\hline (CHECKEOBY) & (DATE)
\end{tabular}

TITLE

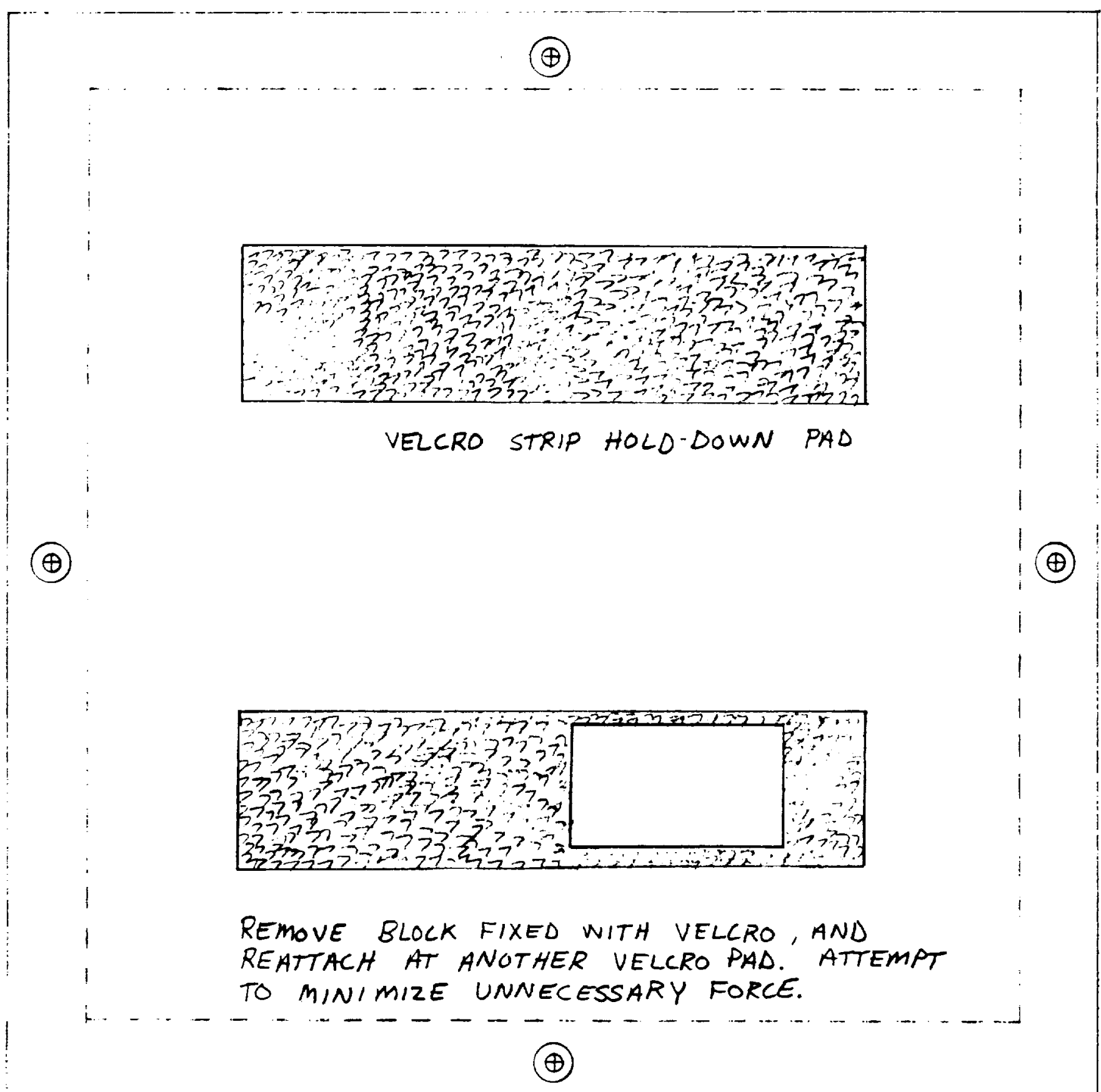


Appendix B

\section{Task Board Modifications and Improvements \\ Brent Guggisberg}


INTEROFFICE MEMORANDUM

19 December 1988

To: Blake Hannaford

FROM: Brent Guggisberg $\because$ !

SUBJECT: Task Board Notes

The general approach to designing new tasks has been experimental. An attempt to create new tools and techniques along with their evaluation has led to generic applications which are closely related to scenarios for space station environments. The tasks can be used for evaluating and studying human performance parameters using the Advanced Teleoperator System. The results will provide valuable knowledge needed for enhanced capabilities in telerobotics.

According to a current NASA report on status and future directions for telerobotics research and development programs, the following areas were identified as possible subjects for developing data to support international standards: (1) task boards (2) system performance measures on task boards (3) human performance measures on task boards with teleoperation and (4) autonomous-telerobotic-teleoperated performance comparisons. Standardization of task boards will allow different techniques and technologies to be evaluated against a common set of tasks. This will also permit the selection of consistent and compatible approaches to telerobotic applications as well as establish common measures of performance.

The purpose of this memo is to document the unique set of tasks which has recently been developed at JPL. A brief outline of the task description as well as performance objectives are discussed. Sketches of the task components and photographs of the completed tasks are provided. 


\section{PEG-IN-HOLE TASR}

Two versions of the peg-in-hole task have been developed. The first version had different sized chamfers and tolerances for each hole, as shown in Figure 1. This version was fabricated from 6061-T6 aluminum with a 303 stainless steel peg. Figure 2 shows a drawing of the aluminum matrix set-up. Preliminary experiments using the peg-inhole task for measuring operator performance have shown that chamfers do not improve operator efficiency. Other tests showed no significant difference in task completion time for the different hole tolerances.

A second version was machined without chamfers, but with tighter tolerances (a 1.0000 inch peg in a 1.0005 inch hole). The new design features hardened sleeves that can be easily changed or modified (see Figure 3). The 9-hole matrix block was made from 7075-T7 aluminum because it is the "toughest" aluminum avallable. In other words, the material has a very high tensile strength, but not necessarily a large hardness value. 7075-T7 aluminum is also "easy" to machine. The rear cover plate (used to keep the sleeves in place) was made from 6061-T6 because this aluminum costs less and usually proves more readily available. The sleeves were made of A-286 tool steel. Sleeves are better for holding tight tolerances because they can be fabricated on a lathe and honed to achleve the proper tolerance. Flight fasteners are also made of this material. A-286 can be heat treated to obtain a higher hardness value, but heat treating was not required for this application. In addition, A-286 steel will not rust (an important requirement for tight tolerance applications) and has a much higher Rockwell hardness value than the machine ground 303 stainless steel peg. 


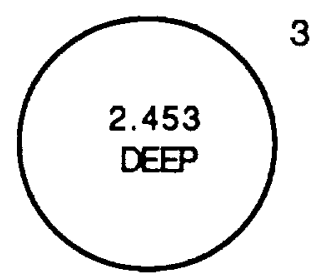

TOP: 1.0030

BOTTOM: 1.0032

NO COUNTERSINK

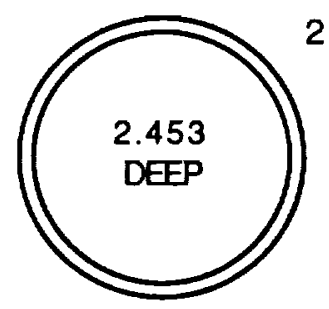

TOP: 1.0028

BOTTOM: 1.0032

1.062 DIA. COUNTERSINK

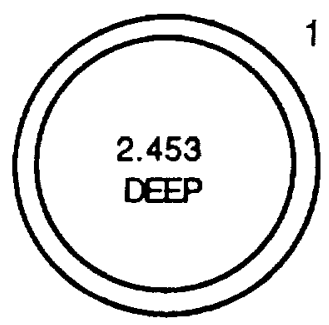

TOP: 1.0032

BOTTOM: 1.0032

1.125 DIA. COUNTERSINK

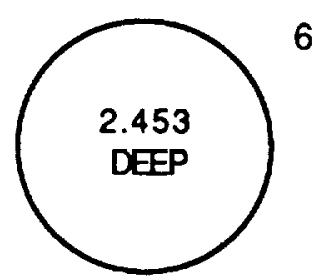

TOP: 1.0019 BOTTOM: 1.0019 NO COUNTERSINK

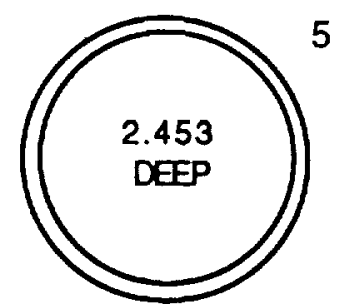

TOP: 1.0018 BOTTOM: 1.0016 1.062 DIR. COUNTERSINK

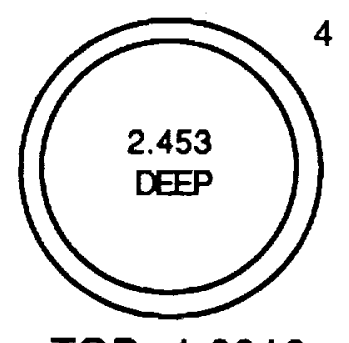

TOP: 1.0016

BOTTOM: 1.0016

1.125 DIR. COUNTERSINK

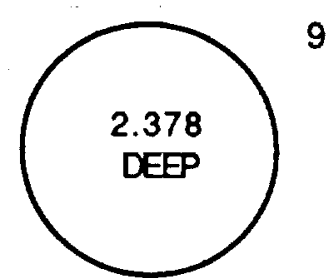

TOP: 1.0007 BOTTOM:1.0005 NO COUNTERSINK

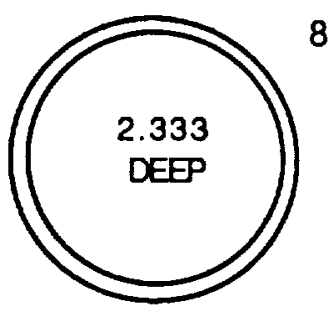

TOP: 1.0006

BOTTOM: 1.0004 1.062 DIA. COUNTERSINK

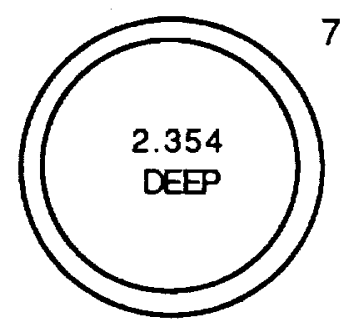

TOP: 1.0006

BOTOM: 1.0004

1.125 DIA. COUNTEASINK

\section{PEG DIMENSIONS}

\section{DIAMETER}
0.9974
0.9977
0.9982
0.9988

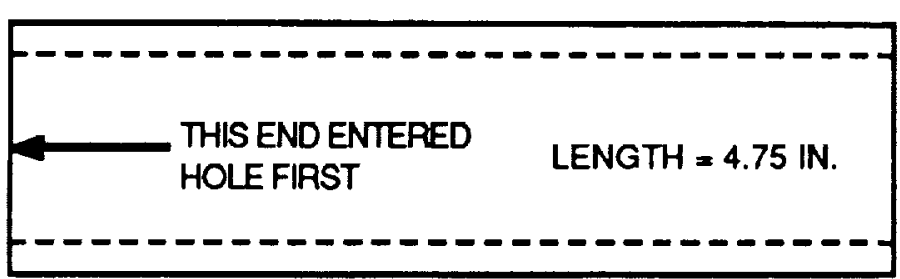

DIAMETER

0.9944

0.9950

0.9964

0.9965 

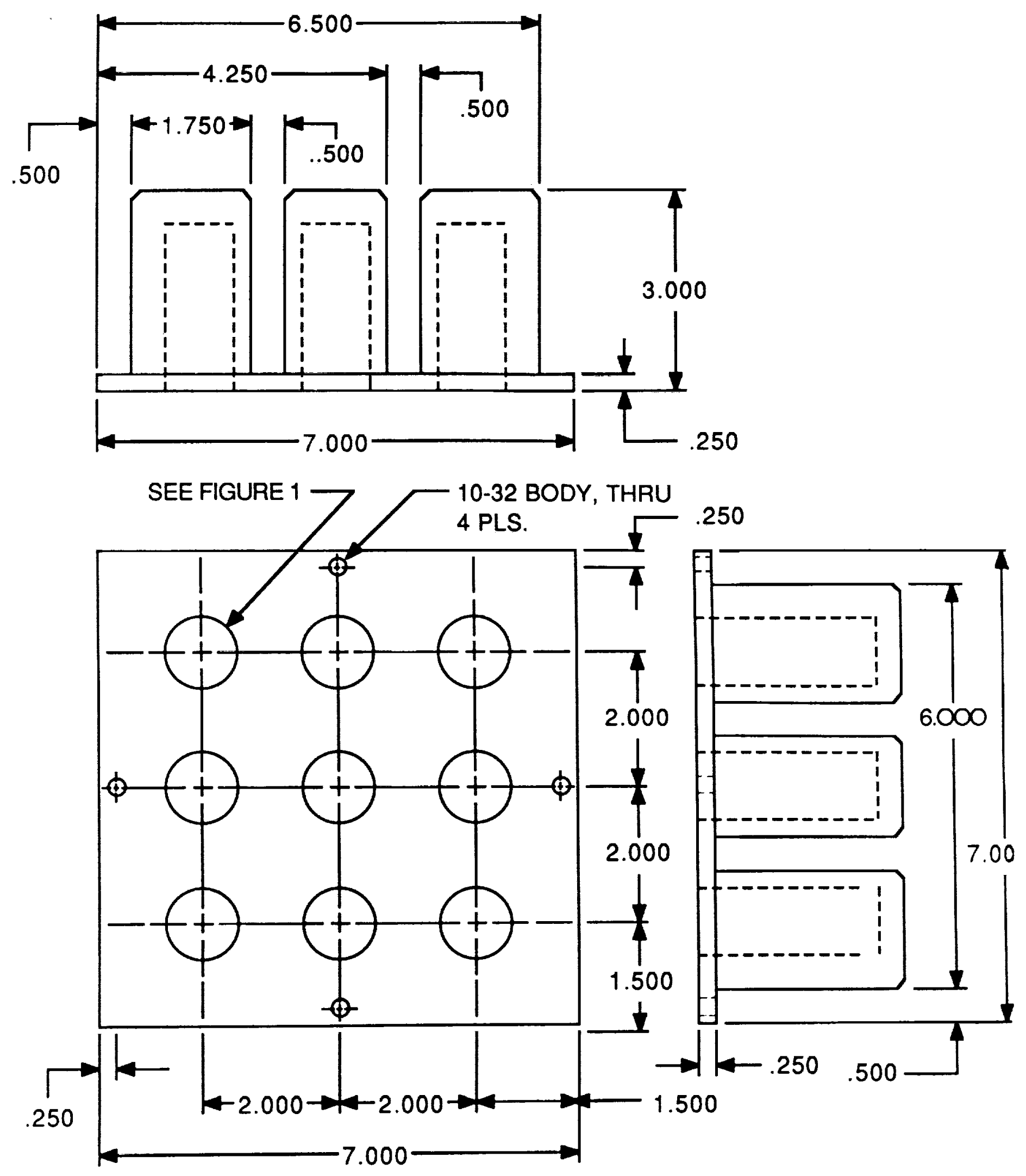

FIGURE 2: ORIGNINAL PEG-IN-HOLE TASK MODULE 

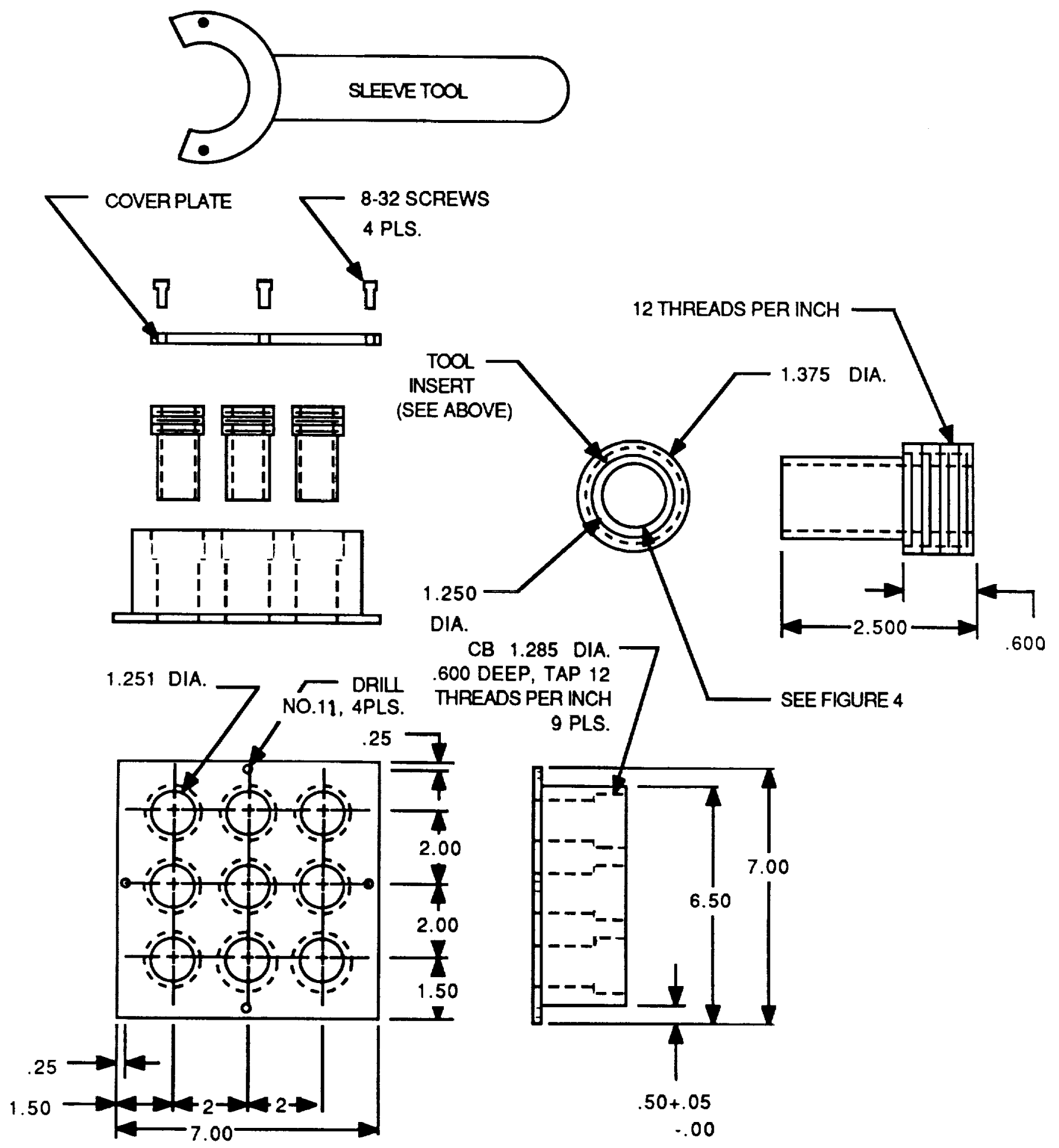

FIGURE 3: NEW PEG-IN-HOLE TASK MODULE 


\section{SLEEVES WERE MACHINED WITH THE FOLLOWING DIMENSIONS}
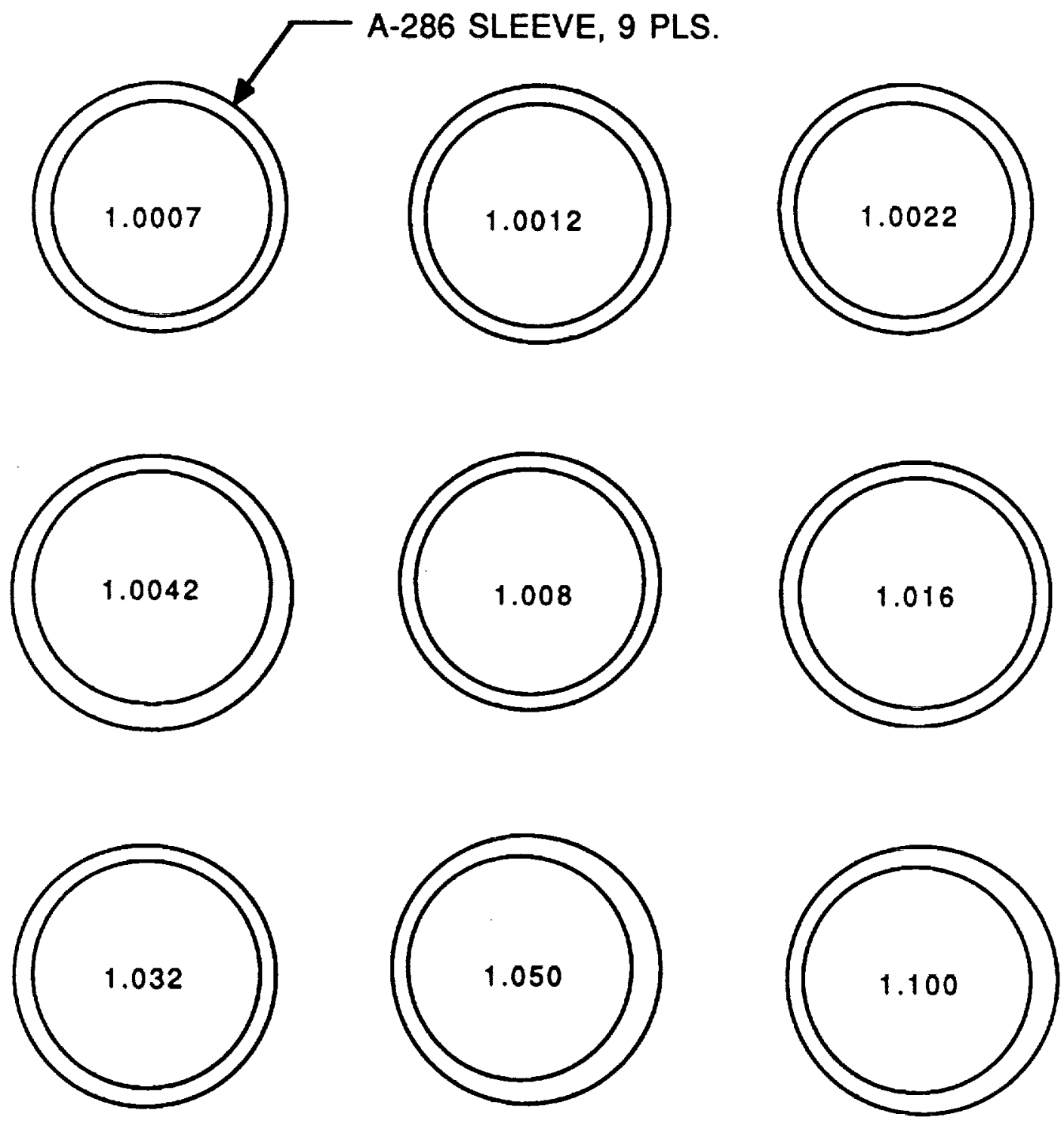

$1.0002,1.0003$, and 1.0006 in. sleeves were also machined.

The peg has a diameter of $1.0002 \mathrm{in.}$ and a length of $4.75 \mathrm{in}$.

FIGURE 4: NEW PEG-IN-HOLE TASK DIMENSIONS 
The following material selection possibilities for the peg-in-hole task were also considered:

- Use the original peg-in-hole 9-hole matrix block and add A-286 tool steel sleeves. 303 or 304 stainless steel for the peg.

o Use the original peg-in-hole 9-hole matrix block and add 4130 steel sleeves. Case hardened or tool steel for the peg.

- Use the original peg-in-hole 9-hole matrix block and add bronze or brass sleeves. 303 or 304 stainless steel for the peg.

- Fabricate an entirely new block from 4130 steel with no sleeves. Case hardened or tool steel for the peg.

- Fabricate an entirely new block from 303 or 304 stainless steel with no sleeves. Bronze or brass for the peg.

It is important to remember that the block/sleeve and the peg must exhibit proper disparity between each other to prevent seize-up. In other words, one must be "hard" and the other must be "soft". For example, a 303 stainless steel block with a 303 stainless steel peg is not a possibility because gouging, gulling, and cold welding will occur. Cold-worked steels are also not acceptable for tight tolerance applications because they do not machine to a smooth surface. In addition, a 9-hole matrix block made from 4130 or stainless steel would be approximately 2.5 times heavier than one made from aluminum. This weight factor would make it very hard and clumsy to change the module around the task board. Moreover, 4130 will rust in normal laboratory conditions. 


\section{ELECIRICAL CONNECTORS TASK}

The following four electrical connectors are mounted in the task window as shown in Figure 5:

(1) 3-pole electrical socket (Hewlett Packard 1251-2357).

(2) 2-pole flanged electrical inlet (General Electric 4343-5).

(3) 0.25 inch head-phone style socket.

(4) Cannon 15 pin rectangular connector.

A corresponding plug attaches to each socket at one end and terminates under a clamp within the task module at the other end.

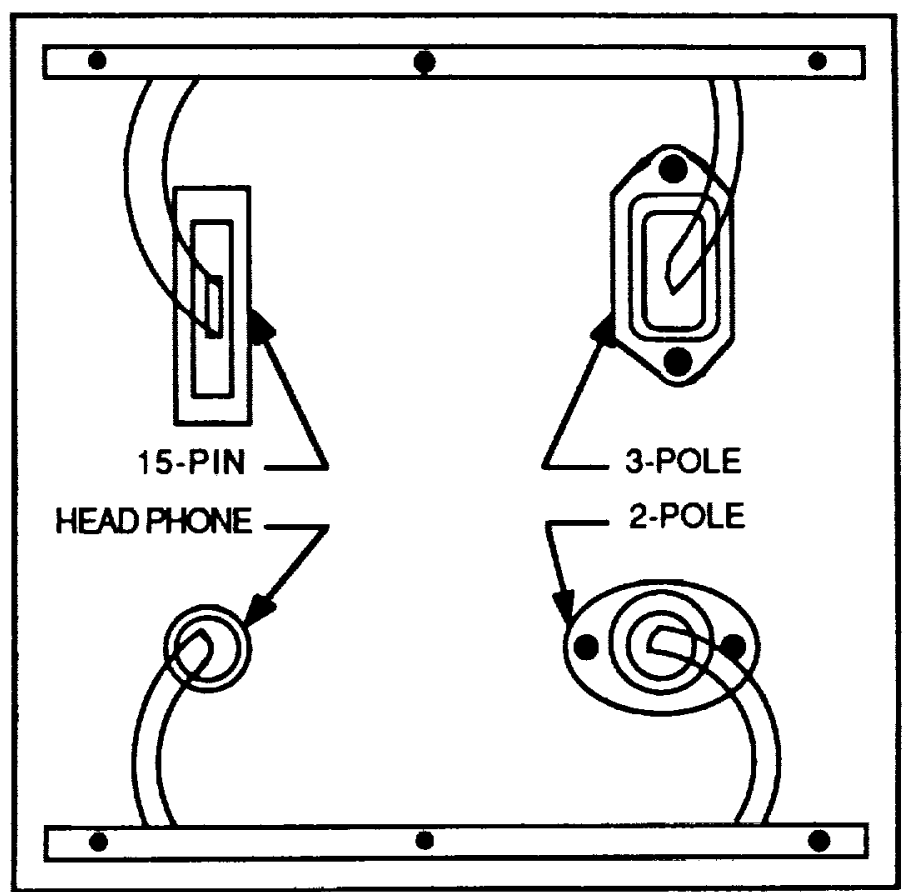

FIGURE 5: ELECTRICAL CONNECTORS TASK 


\section{BAYONET CONNECTOR TASK}

This task involves the mating and unmating of a bendix PT06A-2016S/16P electrical connector. A white line is painted on the socket face in order to "see" the position of the notches for locking the socket to the plug. The top camera view of the task board is used for checking the connector's orientation. A square grip was fitted to the round connector for better handling as shown in Figure 6 below.

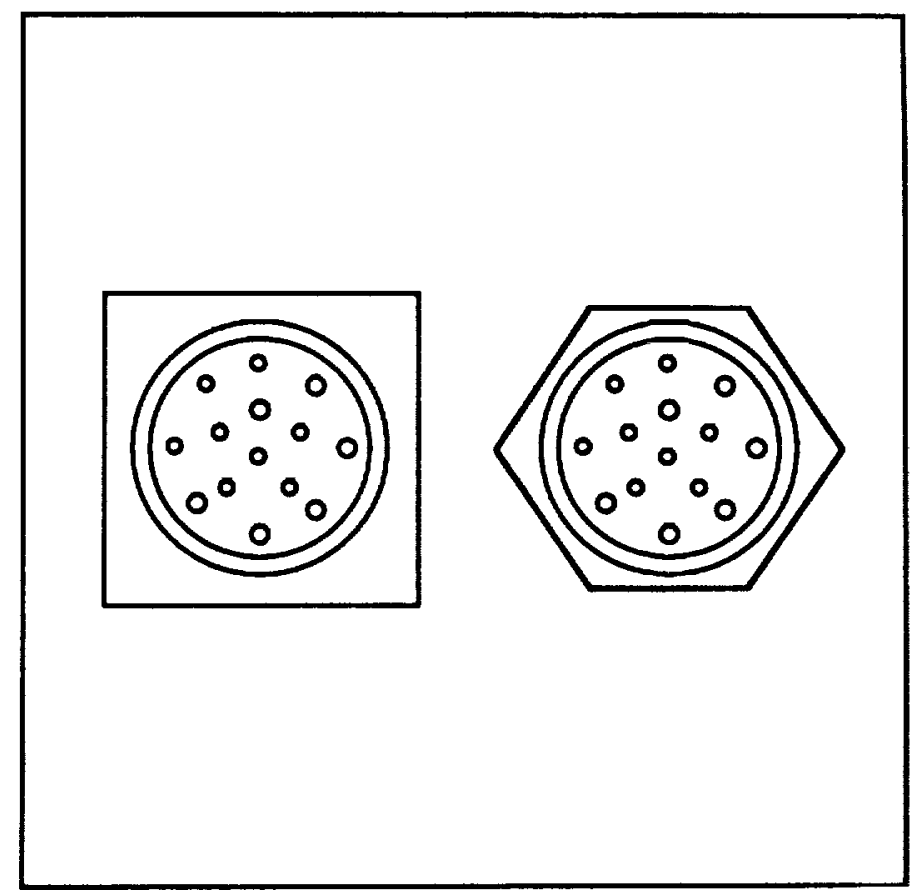

FIGURE 6: BAYONET CONNECTOR TASK 


\section{VELCRO TASK}

An effort is made to minimize unnecessary force while removing and attaching the different sized blocks.

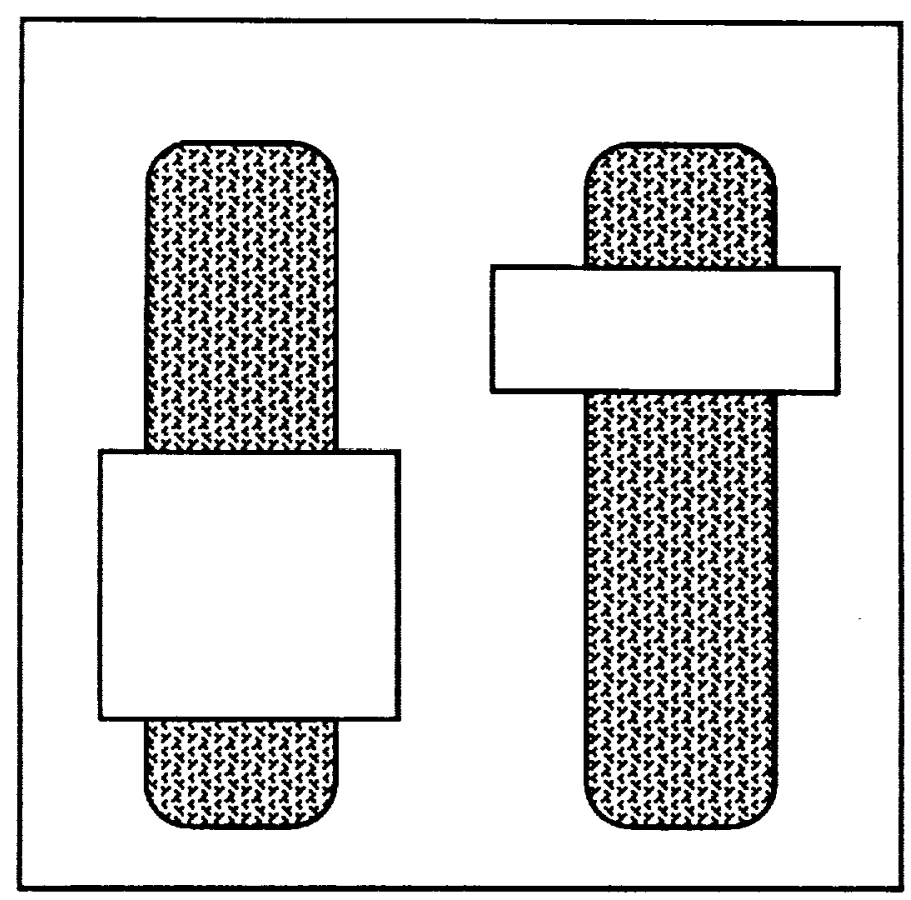

FIGURE 7: VELCRO ATTACHMENT TASK 


\section{CIRCUIT BOARD RPYQVAL TASK}

This task contains a 6.5 inch deep $\times 6.03$ inch width circuit board rack (Buckeye STP BPR 6001-63N) with adjustable height. The rack has 12 board capacity and 0.348 card spacing. The task features a latching door with indexing hinges; see Figure 8. Two stainless steel sliders (Figure 9) rotate through two corresponding Delrin guides (Figure 10). The guides employ spring loaded ball plungers (see manufactures parts list on the attached page) that detent through holes in the slider. The intensity of the indexing can be changed by adjusting the ball plungers .

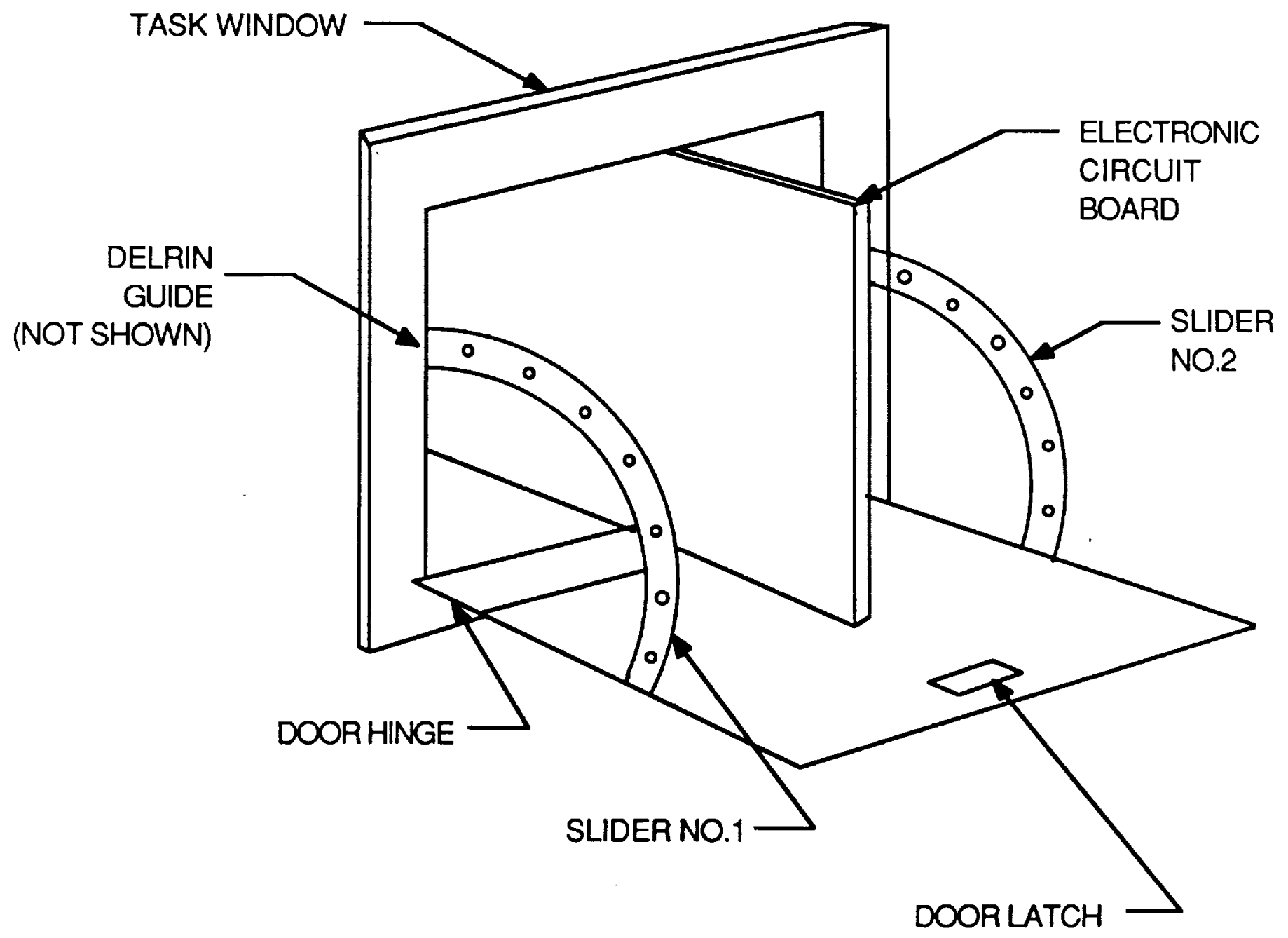

FIGURE 8: CIRCUIT BOARD REMOVAL TASK 


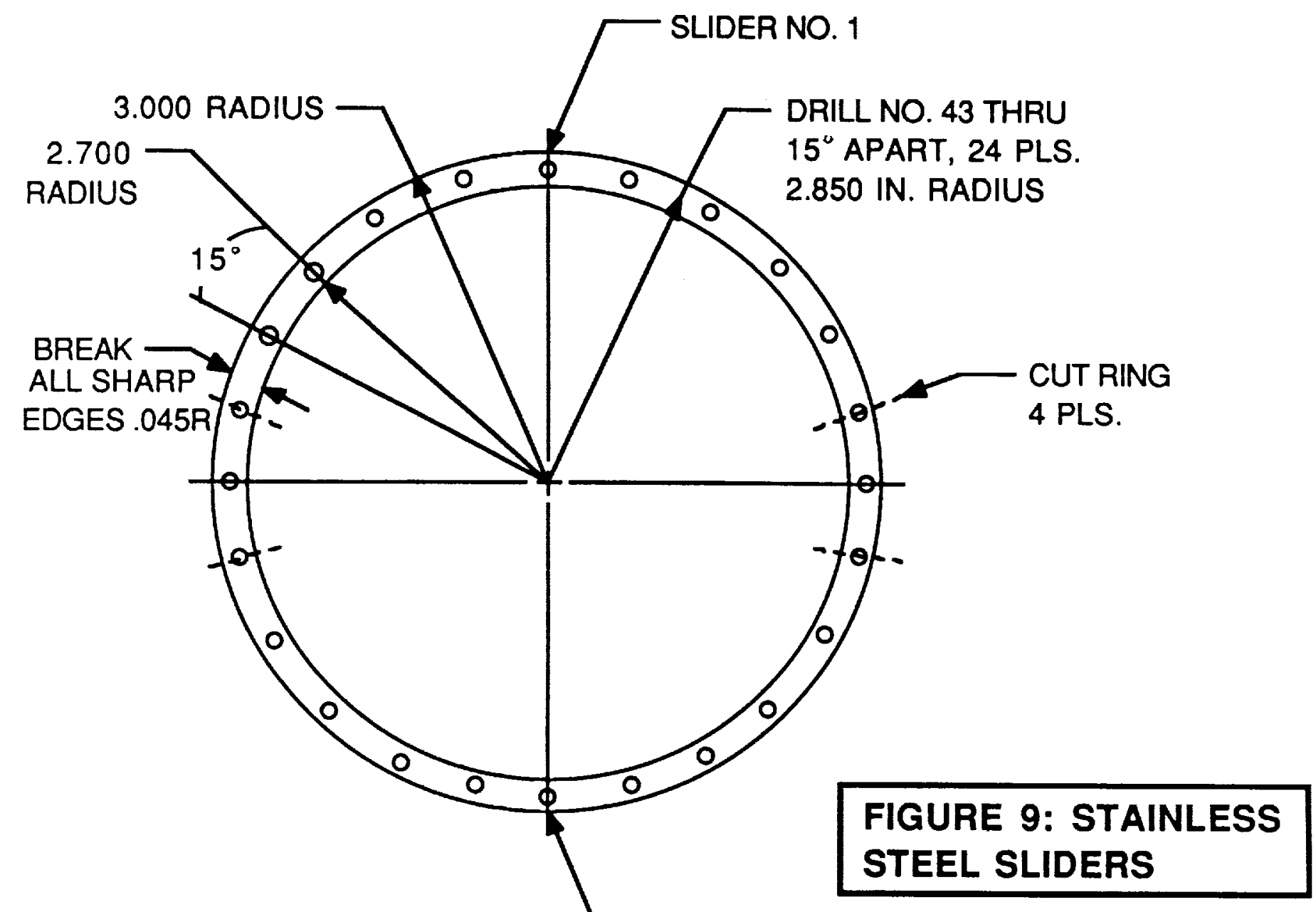

SLIDER NO. 2

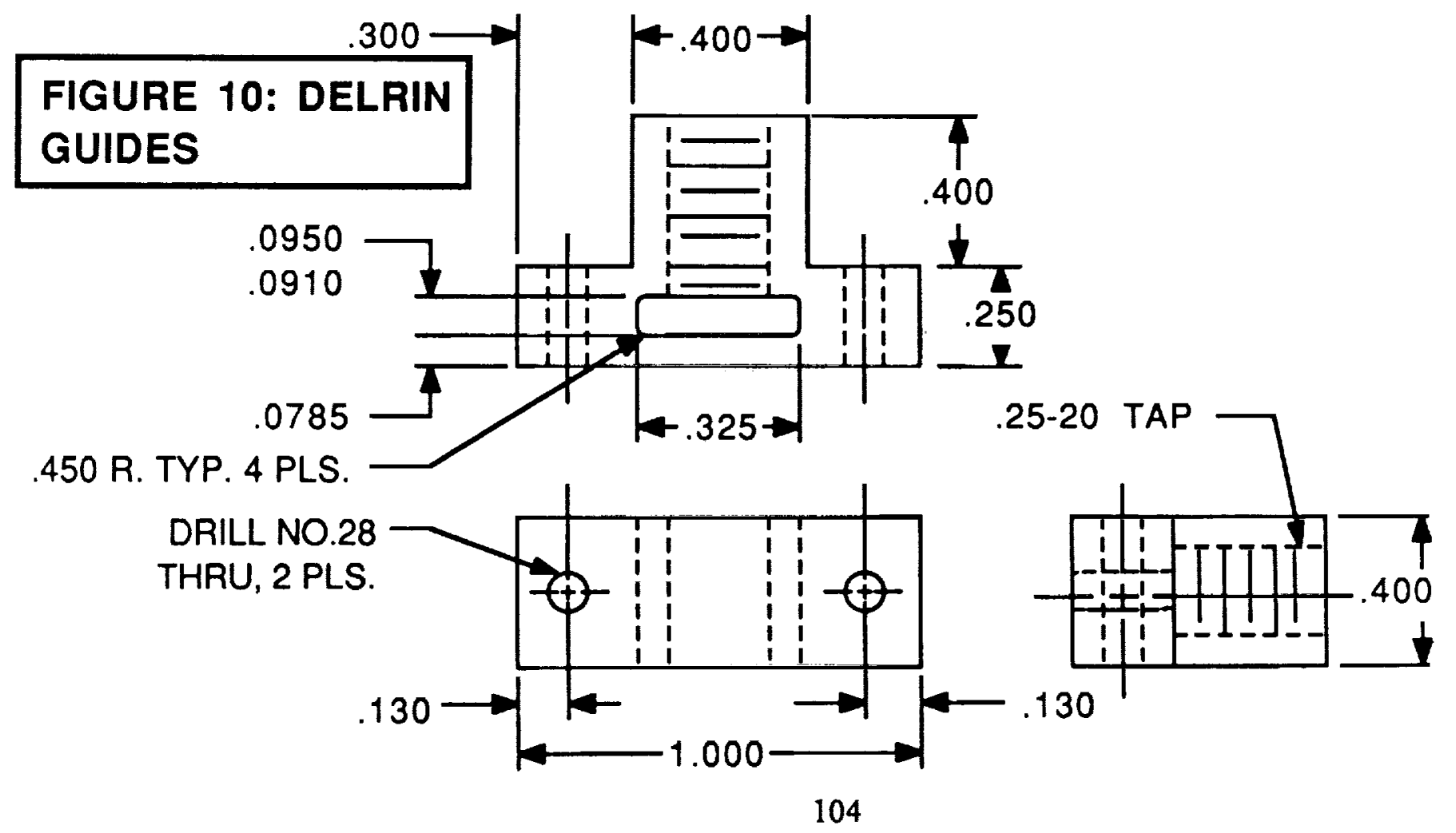




\section{CRANK TASKS}

Two types of cranks are used on the task board. The first crank has an extension arm and a knob while the other uses a simple one inch diameter plastic knob. A nylon bushing with a 10-32 pan head screw are used to attach aluminum knob to the aluminum handle. Both cranks utilize a rotary switch with adjustable indexing (2 to 12 positions) every 30 degrees for "positive action response" to the operator.

An attempt is made to rotate the cranks as smoothly as possible in order to minimize unnecessary force. The crank task, shown in Figure 11 below, operates throughout an entire 360 degree circle while the knob task, shown in Figure 12, functions only within a 300 degree arc. Numbers are engraved at each point of index.

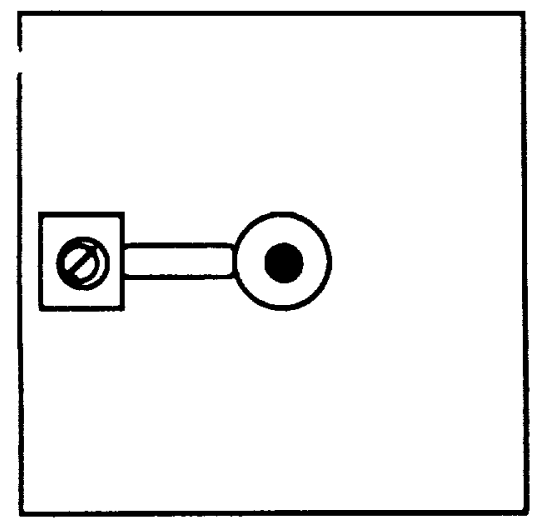

FIGURE 11: CRANK TASK

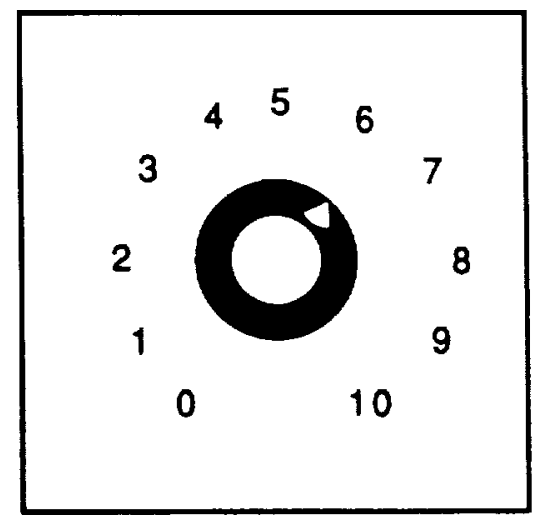

FIGURE 12: KNOB TASK 


\section{PLANAR CONTACT}

This task, displayed in Figure 13, includes a speclal "tool" for making "planar contact" with a surface within the task module. The tool pushes against four springs mounted in the rear of the task structure. When the tool makes complete surface contact with the rear task plate, four micro switches are engaged which light up four red indicator lamps. If any part of the tool plate is not making absolute contact with the spring/switch plate, the matching light will not turn on. Figure 14 shows the tool handle which is an exact inverse of the "smart-hand" grippers.

Two electrical component boxes are mounted on the back side of the task structure which house two nine volt batteries and all electrical wiring. A switch mounted outside one of the electrical component boxes will turn on all lights at once for testing purposes.

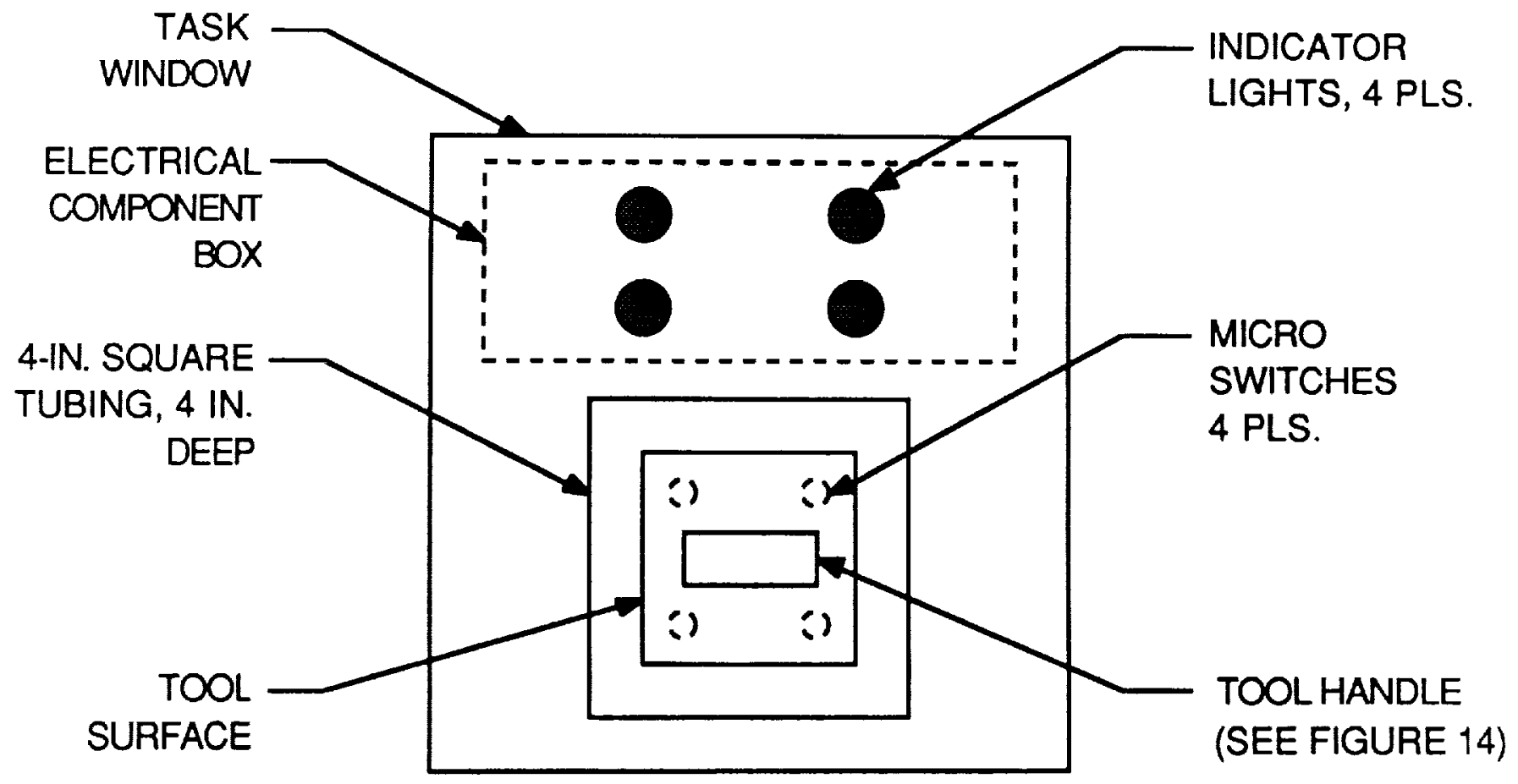

FIGURE 13: PLANAR CONTACT TASK 


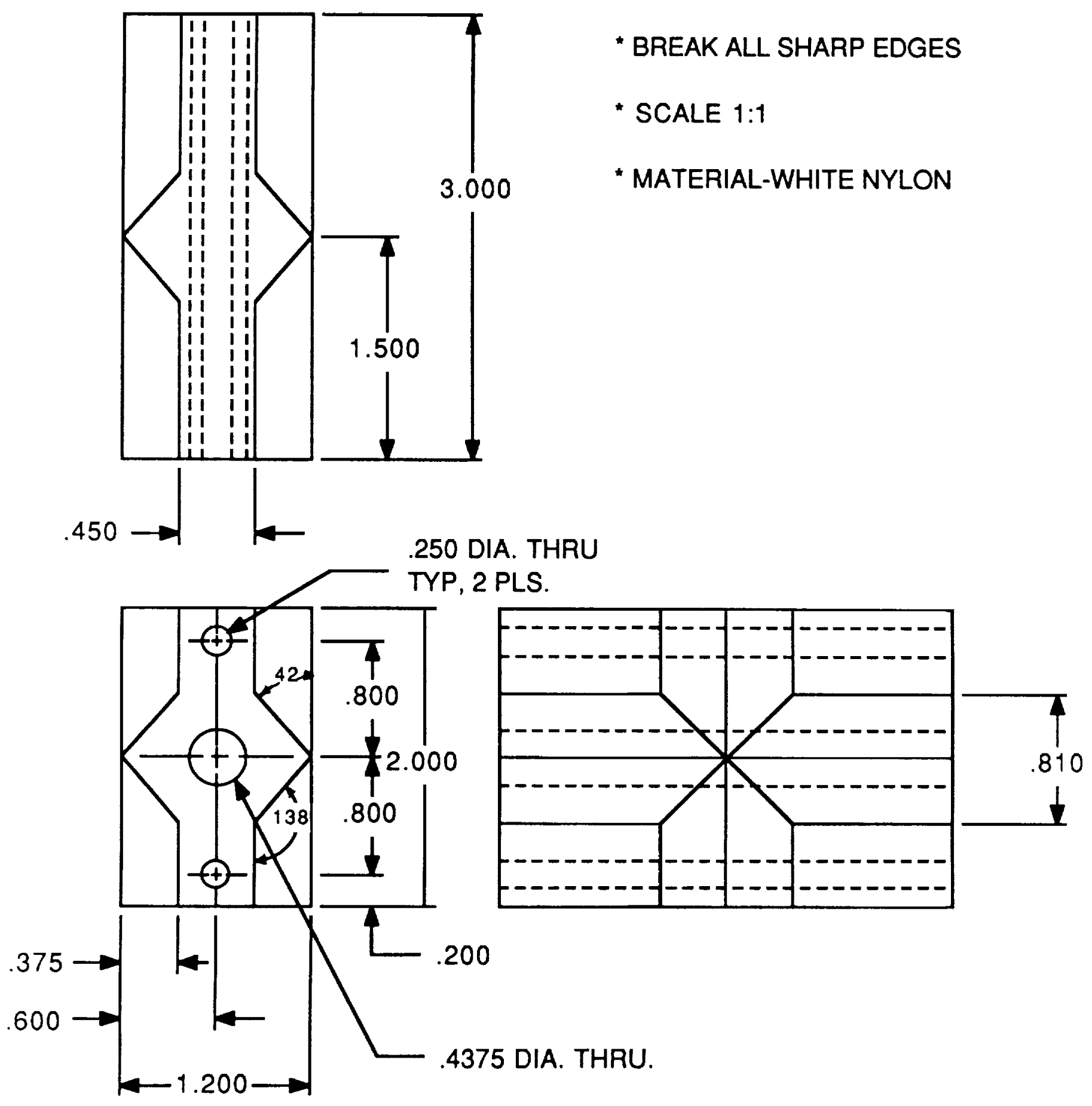

FIGURE 14: NYLON TOOL HANDLE 


\section{FASTENER REYOVAL/ATTACHDIENT TASK}

A distinctive set of tools was procured for this task. Modified ratcheting drivers are used to screw various sized bolts into corresponding threaded holes. The drivers have custom nylon tool handles similar to the one in Figure 14.

The fasteners are coupled with one task plate (Figure 15) while the driver and sockets are stored in a second task plate (Figure 16). Sockets can be easily changed without extra assistance. Figure 17 shows a drawing of the socket containers. The driver also features a special T-shaped extension for changing the ratchet direction. This can also be performed using only the teleoperator system.

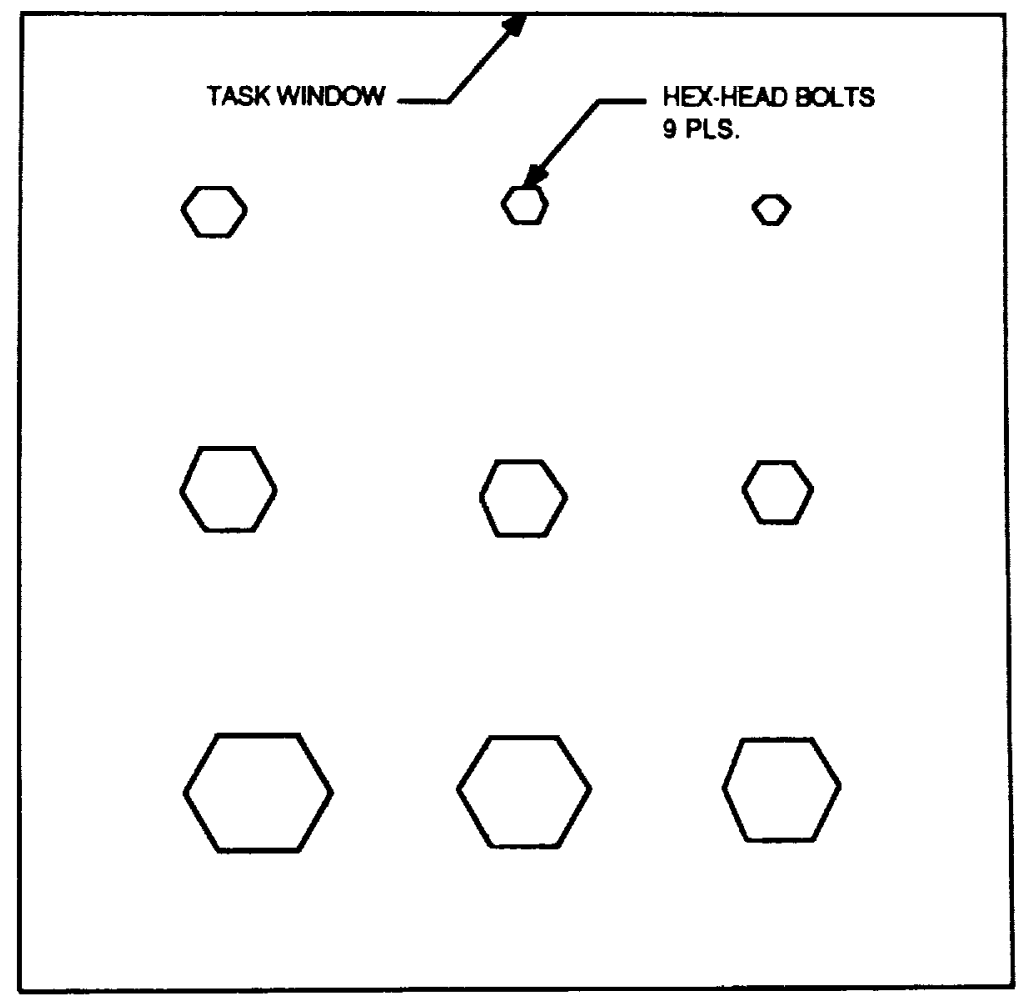

FIGURE 15: FASTENER REMOVAL/ATTACHMENT TASK 


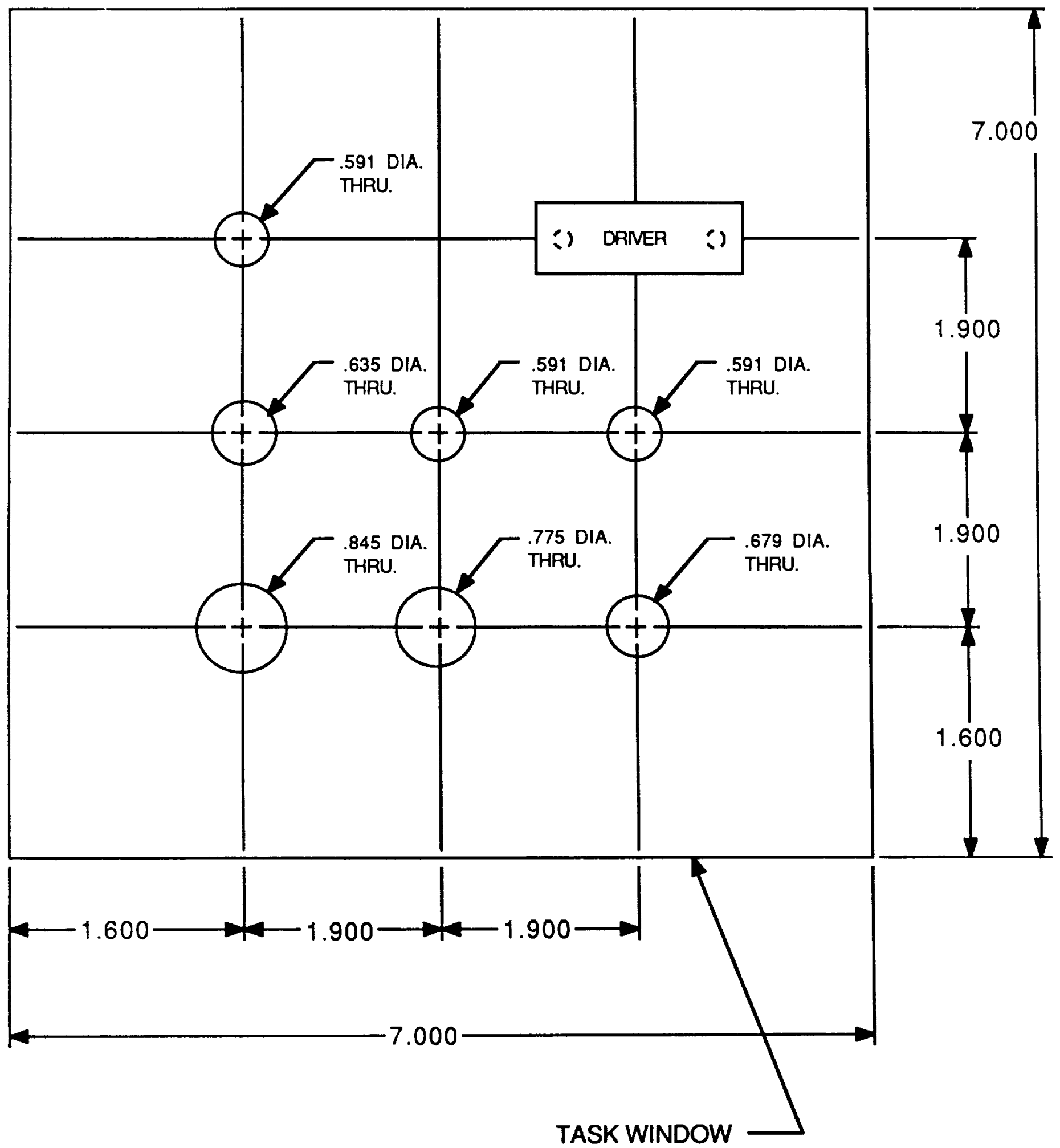

FIGURE 16: DRIVER/SOCKET HOLDER SUBTASK 


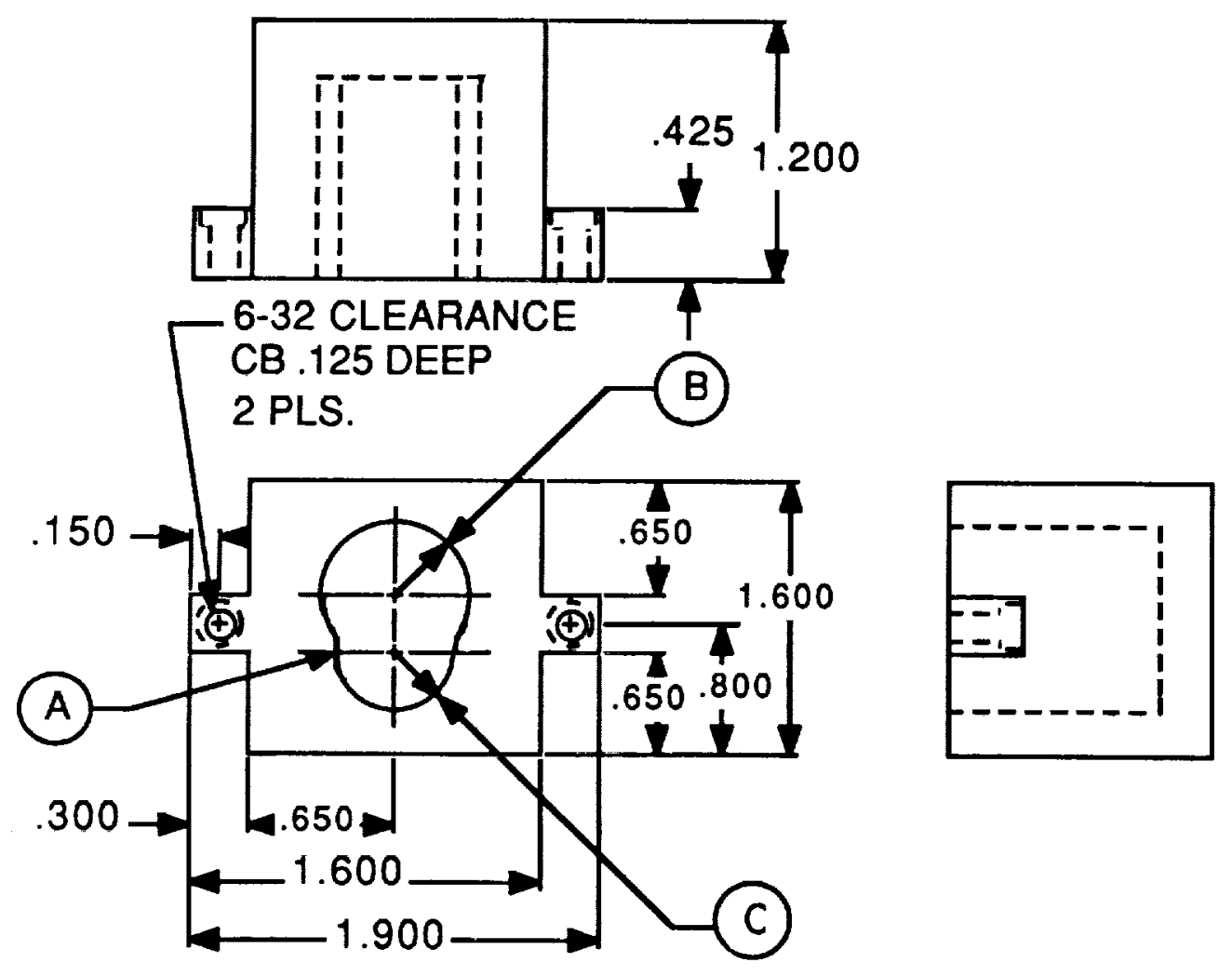

(A) MACHINE AS SHOWN

(B) R.423 R.388 $\quad$ R.340 R.318 R.296 R.296 R.296 (1.000 DEEP)

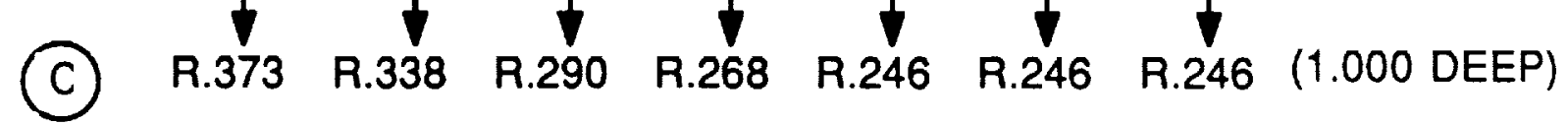

MATERIAL: ALUMINUM

MAKE ONE EACH PER DIMENSIONS ABOVE

FIGURE 17: SOCKET HOLDERS 
Neoprene Rubber Coating for "Smart-Hand" Grippers and Task Board Components.

\author{
Numerous components on task board as well as the "smart-hand" \\ grippers were coated with a resilient material that provides high \\ friction gripping. Plastic Dip, Solathane, Color Guard Tough Rubber \\ Coating, and neoprene rubber were all tested, The neoprene rubber \\ turned out to be the most durable covering. An outside vendor was used \\ to apply the .055 inch thick coating.
}




\section{Appendix C}

\section{Task Sequence Descriptions}




\section{TASK SEQUENCE DESCRIPTIONS}

1. Velcro Blocks

1.1 Attach and detach both blocks; tap mark, grasp small block, detach, attach block, release grasp, tap on small block, grasp large block, detach, attach block, release grasp, tap on large block, release grasp, tap mark.

1.2 Exchange block positions: tap mark, grasp small block (at position "A"), detach, move to side (position "B"), attach, release, tap, grasp large block (position "C"), detach, move to position "A", attach, release, tap, grasp small block (at position "B"), move to position "C", attach, relcase, tap small block, tap mark.

2. Peg-in-Hole Matrix

2.x Inscrt peg in hole $\mathrm{x}$ : (nine holes, subtasks $2.1-2.9$ ) start with peg in gripper, tap) mark with peg tip, move to hole $x$, insert peg, release grasp, tap on end of peg, regrasp, extract peg, move to mark, tap mark.

3. Electrical Connectors

3.x Unmate and mate connector $x$. Tap mark, move to connector $\mathrm{x}$, grasp connector, unmate, move to mark, tap mark with connector, move back to receptacle, mate, release, tap connector, move back to mark, tap. Iicy: (1) 3-prong chassis power connector, (2) 25-pin RS-232 connector (3) 1/4" headphone plug.

4. Bendix Connector

4.1 Unmate and mate connector. Tap mark move to left receptacle (where connector is mated), align jaws, grasp locking ring (via fixture), rotate counter clockwise 90 deg, remove connector, move to right receptacle, align connector, rotate locking ring clockwise $90 \mathrm{deg}$, ungrasp, move to mark, tap.

Subject Instructions. After practice, and before beginning the data taking, the subjects were instructed to perform the tasks as fast as possible wlile minimizing the amount of force used. In addition, the subjects were asked to avoid making "errors" of specific types defined in Table 3. 


\title{
Appendix D
}

\section{Operator Skill Testing and Prediction}

\author{
Haya Zak
}




\section{OPERATOR SKILL TESTING AND PREDICTION}

In an attempt to establish a teleoperator skill test that would constitute a prediction criterion for operator's performance, two motor control tests were designed: the "Star Test" and the "Circle Test."

\section{Test Descriptions}

Both tests were implemented on an IRIS 2400 graphics computer. The Star Test had the following features: A figure of a star was presented to the subject, consisting of an inner star outline and an outer star outline separated by approximately 0.25 inches (Fig. D-1). The objective was to trace between the two star outlines (called "inside the star") with a moused-controlled cursor, while maintaining maximum tracing speed and accuracy. Speed was measured in "frames" (in one minute there are 1440 frames), an indicator of the total time required to complete an end-to-end tracing of a star. Accuracy was measured as "total length" - the length (inches) of the cursor's path in tracing inside and outside the star. The optimal length in tracing the star was 34.38 inches, thus any "total length" recording that exceed this value is an indication of whether and by how much the subject deviated from the optimal path. The recording of completion time and performance accuracy started as soon as the subject pushed the mouse button. As long as the cursor moved inside the star, the path traveled by the cursor was filled with a red color, serving as a positive feedback to the subject. Once deviation from the inner path occurred, the red color would stop filling the inner path and the green line marking the path traveled by the cursor went outside the star, serving as a negative feedback. In order to "get on the right track again," the subject had to get on the inner path exactly on or before the point of deviation, marked by the end of the red filled part.

The Circle Test consisted of a target red circle (approximately 1 inch in diameter) which was following a circular (invisible) path (approximately 10 inches in diameter) with a speed of 22.35 cycles per minute. Another "small" blue circle (approximately 0.5 inch in diameter) controlled by the mouse, was the "hunter." The objective was to keep the small circle on target (the big circle) as the target moved along its path. Accuracy was measured in this case as time on Target (percent of total time).

\section{Experimental Procedures}

The 5 subjects performed 32 repetitions of the Star Test and 60 repetitions of the Circle Test. The high number of repetitions was designed to bring the subjects to a reasonably reliable level of performance, i.e., where the "leveling off" of the learning curve occurs. The Star Test was further elaborated to include various mouse orientations either $0,90,180$, or 270 degrees in relation to the hand's grasp position. Subjects were required to change the mouse orientation every repetition, such that 8 repetitions were performed for each orientation. 


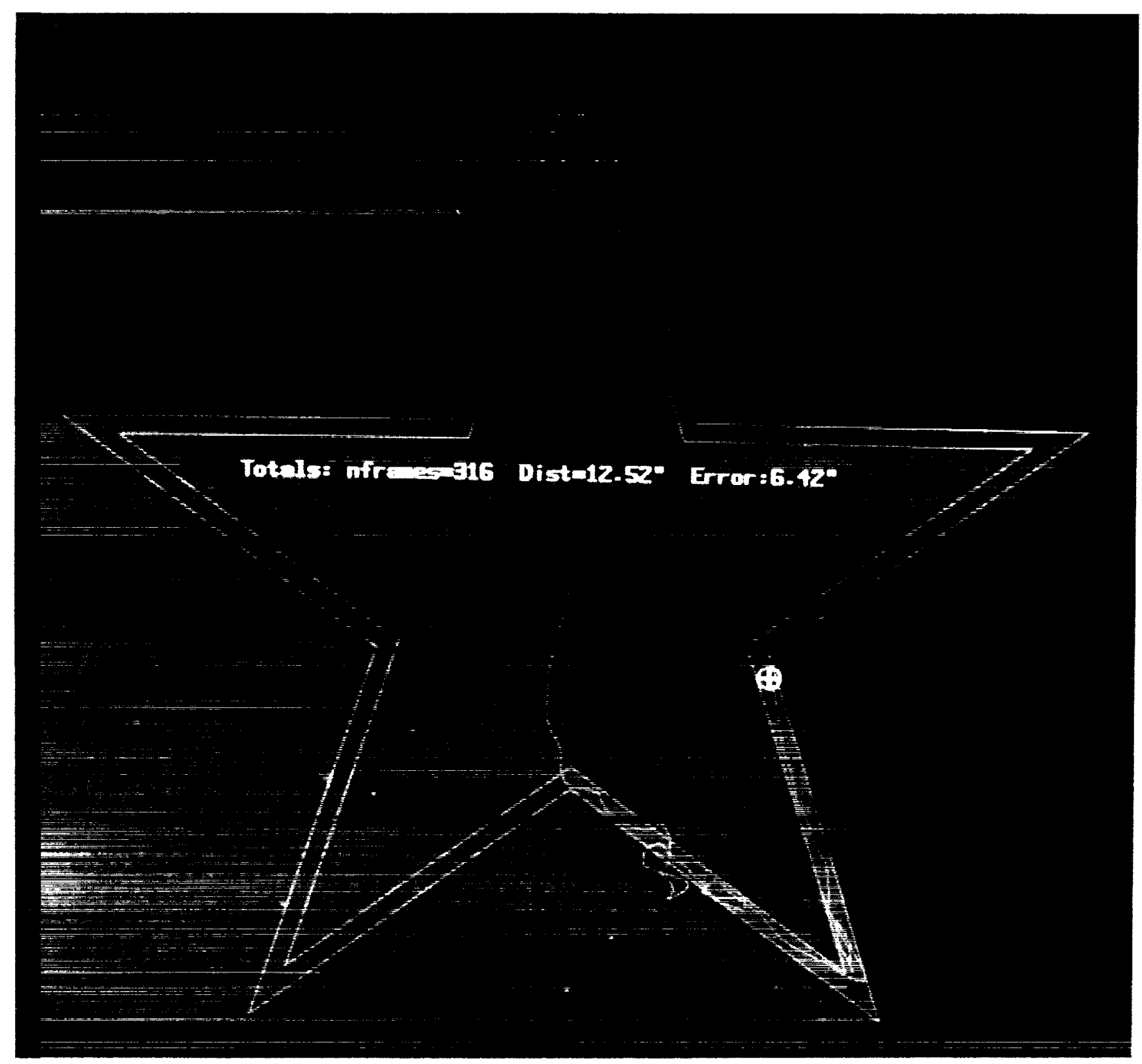

Figure D-1. Screen image from the star tracing test used in motor control pre-testing. The subjects used a mouse to trace between the two star outlines. The task was made more difficult by imposing rotations of $90,180,270$ and 0 degrees between mouse motion and cursor motion.

\section{ORIGINAL PAGE}

BLACK AND WHITE PHOTOGRAPH 


\section{Data Analysis}

The two mean performance measures for the Star Test (total length and completion time) were taken for each subject, then correlated with teleoperation performance measures (time, force, and errors). A summary of results is shown in Tables D.1-D.8. The Pearson product-moment correlation was computed for each test-teleoperation performance measurcment pair. In this method, the significance of each correlation is determined via the $t$ test (where $t=\frac{r \sqrt{n-2}}{\sqrt{1-r^{2}}}$ ). For 4 subjects (at alpha=0.05), the value required for significance is $t=0.05$. The numbers in each square indicate the number of significant correlations out of the total number of correlations (the latter in parenthesis).

Table D.1 summarizes our findings for the Star Test. The data breakdown is by mouse orientation, by mode (FFB or MANUAL) and by task (1-4 and ALL). Since other kinds of analysis performed on this data show task 4 to be quite different in nature from tasks $1-3$, it was decided to treat task 4 as a spccial category, while tasks $1-3$ could be averaged together to form the ALL category. From table D.1 it appears that there were no significant correlations, i.e., 0 out of 80 , for the MANUAL mode, while 6 correlations out of 88 were found to be significant in the FFB mode. Nevertheless, 6(88) may still be random results (for alpha=0.1), therefore, it is difficult to conclude that the FFB mode contributed to the results. Within the FFB mode, the most "promising" orientation appears to be 180 degrees, but again $3(22)$ significant correlations may be due to chance alone. No particular task appears to be taking the lead in terms of significance. The ALL category seems to correlate slightly more than the individual tasks, yet this may very well be a chance result as wcll. Ovcrall, only 6(168) scored significantly, thus, the results do not allow us to reject the null hypothesis at alpha $=0.05$.

Talbles D.4-D.8 summarize the Star Test results for each task (Table D.5, task 1; Table D.6, task 2; Table D.7, task 3; Table D.8, task 4; Table D.4 arcrage of tasks 1-3). The now information gained by this representation is that "time" for the Star Test may be a better predictor of telcoperation measures: 7(84) were significant for "time" while 0(84) was recorded for "length." The breakdown of ERRORS by task is not available, although it does exist for all tasks (1-4). Since 2(8) correlations with ERRORS were found to be significant, this is a promising result that should be explored in future experiments.

Table D.2 summarizes the results for the Circle Test. While for the FFB mode there are no significant corrclations found, i.e., 0(5) for the TIME and ERRORS, 2(4) correlations wore significant for the FORCE measure. The latter result offers good support for rejecting the null hypothesis. Once again, comparison of the FFB mode to the MANUAL mole results in FFB outperforming the MANUAL in terms of the number of significant corrclations.

Table D. 3 is yet another summary of results for the Circle Test. 


\section{Concluding Remarks}

The goal of establishing a valid teleoperator skill test has not yet been achieved based on the results of the above experiment. Further research is needed to investigate the potcntial of the Star Test and the Circle Test for being reliable teleoperator skill tests. 
TABLE D.I

\begin{tabular}{|c|c|c|c|c|c|c|}
\hline & TASK & STAR $0^{\circ}$ & STAR $90^{\circ}$ & STAR $180^{\circ}$ & STAR $270^{\circ}$ & total \\
\hline \multirow{6}{*}{ 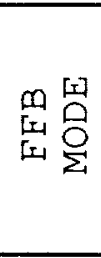 } & 1 & $0(4)$ & $0(4)$ & $0(4)$ & $0(4)$ & $0(16)$ \\
\hline & 2 & $0(4)$ & $0(4)$ & $1(4)$ & $0(4)$ & $1(16)$ \\
\hline & 3 & $1(4)$ & $0(4)$ & $0(4)$ & $0(4)$ & $1(16)$ \\
\hline & 4 & $1(4)$ & $0(4)$ & $0(4)$ & $0(4)$ & $1(16)$ \\
\hline & $\overline{\mathrm{ALL} *}$ & $0(6)$ & $0(6)$ & $2(6)$ & $1(6)$ & $3(24)$ \\
\hline & subtotal & $2(22)$ & $0(22)$ & $3(22)$ & $1(22)$ & $6(88)$ \\
\hline \multirow{7}{*}{ 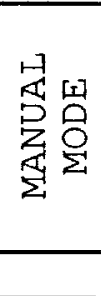 } & 1 & $0(4)$ & $0(4)$ & $0(4)$ & $0(4)$ & $0(16)$ \\
\hline & 2 & $0(4)$ & $0(4)$ & $0(4)$ & $0(4)$ & $0(16)$ \\
\hline & 3 & $0(4)$ & $0(4)$ & $0(4)$ & $0(4)$ & $0(16)$ \\
\hline & 4 & $0(4)$ & $0(4)$ & $0(4)$ & $0(4)$ & $0(16)$ \\
\hline & ALL * & $0(4)$ & $0(4)$ & $0(4)$ & $0(4)$ & $0(16)$ \\
\hline & subtotal & $0(20)$ & $0(20)$ & $0(20)$ & $0(20)$ & $0(80)$ \\
\hline & total & $2(42)$ & $0(42)$ & $3(42)$ & $1(42)$ & $6(168)$ \\
\hline
\end{tabular}

TABLE D. 2

\begin{tabular}{|c|l|c|c|c|c|c|c|}
\hline & & TASK 1 & TASK 2 & TASK 3 & TASK 4 & ALL $^{*}$ & total \\
\hline \multirow{3}{*}{ FFB } & TIME & $0(1)$ & $0(1)$ & $0(1)$ & $0(1)$ & $0(1)$ & $0(4)$ \\
\cline { 2 - 8 } & FORCE & $0(1)$ & $1(1)$ & $0(1)$ & $0(1)$ & $1(1)$ & $2(4)$ \\
\cline { 2 - 8 } & ERRORS & $\mathrm{n} / \mathrm{a}$ & $\mathrm{n} / \mathrm{a}$ & $\mathrm{n} / \mathrm{a}$ & $\mathrm{n} / \mathrm{a}$ & $0(1)$ & $0(1)$ \\
\cline { 2 - 8 } & subtotal & $0(2)$ & $1(2)$ & $0(2)$ & $0(2)$ & $1(3)$ & $2(11)$ \\
\hline \multirow{2}{*}{$\begin{array}{c}\text { MANUAL } \\
\text { MODE }\end{array}$} & TIME & $0(1)$ & $0(1)$ & $0(1)$ & $0(1)$ & $0(1)$ & $0(5)$ \\
\cline { 2 - 8 } & FORCE & $0(1)$ & $0(1)$ & $0(1)$ & $0(1)$ & $0(1)$ & $0(5)$ \\
\cline { 2 - 8 } & Subtotal & $0(2)$ & $0(2)$ & $0(2)$ & $0(2)$ & $0(2)$ & $0(10)$ \\
\hline & total & $0(4)$ & $1(4)$ & $0(4)$ & $0(4)$ & $1(5)$ & $2(21)$ \\
\hline
\end{tabular}

TABLE D. 3

\begin{tabular}{|c|c|c|c|c|}
\hline & TIME & FORCE & ERRORS & total \\
\hline $\begin{array}{c}\text { FFB } \\
\text { MODE }\end{array}$ & $0(5)$ & $2(5)$ & $0(1)$ & $2(11)$ \\
\hline $\begin{array}{c}\text { MANUAL } \\
\text { MODE }\end{array}$ & $0(5)$ & $0(5)$ & $\mathrm{n} / \mathrm{a}$ & $0(10)$ \\
\hline total & $0(10)$ & $2(10)$ & $0(1)$ & $2(21)$ \\
\hline
\end{tabular}

*ALL includes tasks 1-3 only, except for the correlations with ERRORS where all tasks (1-4) are included. 
TABLE D. 4

\begin{tabular}{|c|c|c|c|c|c|}
\hline & & TIME & FORCE & ERRORS & totaI \\
\hline \multirow{3}{*}{ LENGTH } & FFB & $0(4)$ & $0(4)$ & $0(4)$ & $0(12)$ \\
\cline { 2 - 6 } & MANUAL & $0(4)$ & $0(4)$ & $\mathrm{n} / \mathrm{a}$ & $0(8)$ \\
\cline { 2 - 6 } & Subtotal & $0(8)$ & $0(8)$ & $0(4)$ & $0(20)$ \\
\hline \multirow{3}{*}{ TIME } & FFB & $0(4)$ & $1(4)$ & $2(4)$ & $3(12)$ \\
\cline { 2 - 6 } & MANUAL & $0(4)$ & $0(4)$ & $\mathrm{n} / \mathrm{a}$ & $0(8)$ \\
\cline { 2 - 6 } & Subtotal & $0(8)$ & $1(8)$ & $2(4)$ & $3(20)$ \\
\hline & total & $0(16)$ & $1(16)$ & $2(8)$ & $3(40)$ \\
\hline
\end{tabular}

TABLE D. 5

\begin{tabular}{|c|c|c|c|c|c|}
\hline & & TIME & FORCE & ERRORS & total \\
\hline \multirow{3}{*}{ LENGTH } & FFB & $0(4)$ & $0(4)$ & $\mathrm{n} / \mathrm{a}$ & $0(8)$ \\
\cline { 2 - 6 } & MANUAL & $0(4)$ & $0(4)$ & $\mathrm{n} / \mathrm{a}$ & $0(8)$ \\
\cline { 2 - 6 } & subtotal & $0(8)$ & $0(8)$ & $\mathrm{n} / \mathrm{a}$ & $0(16)$ \\
\hline \multirow{3}{*}{ TIME } & FEB & $0(4)$ & $0(4)$ & $\mathrm{n} / \mathrm{a}$ & $0(8)$ \\
\cline { 2 - 6 } & MANUAL & $0(4)$ & $0(4)$ & $\mathrm{n} / \mathrm{a}$ & $0(8)$ \\
\cline { 2 - 6 } & subtotal & $0(8)$ & $0(8)$ & $\mathrm{n} / \mathrm{a}$ & $0(16)$ \\
\hline & total & $0(16)$ & $0(16)$ & $\mathrm{n} / \mathrm{a}$ & $0(32)$ \\
\hline
\end{tabular}

TABLE D. 6

\begin{tabular}{|c|c|c|c|c|c|}
\hline & & TIME & EORCE & ERRORS & total \\
\hline \multirow{3}{*}{ LENGTH } & FFB & $0(4)$ & $0(4)$ & $\mathrm{n} / \mathrm{a}$ & $0(8)$ \\
\cline { 2 - 6 } & MANUAI & $0(4)$ & $0(4)$ & $\mathrm{n} / \mathrm{a}$ & $0(8)$ \\
\cline { 2 - 6 } & subtotal & $0(8)$ & $0(8)$ & $\mathrm{n} / \mathrm{a}$ & $0(16)$ \\
\hline \multirow{3}{*}{ TIME } & EEB & $0(4)$ & $2(4)$ & $\mathrm{n} / \mathrm{a}$ & $2(8)$ \\
\cline { 2 - 6 } & MANUAI & $0(4)$ & $0(4)$ & $\mathrm{n} / \mathrm{a}$ & $0(8)$ \\
\cline { 2 - 6 } & subtotal 1 & $0(8)$ & $2(8)$ & $\mathrm{n} / \mathrm{a}$ & $2(16)$ \\
\hline & total & $0(16)$ & $2(16)$ & $\mathrm{n} / \mathrm{a}$ & $2(32)$ \\
\hline
\end{tabular}

TABLE D. 7

\begin{tabular}{|c|c|c|c|c|c|}
\hline & & TIME & FORCE & ERRORS & total \\
\hline \multirow{3}{*}{ LENGTH } & FFB & $0(4)$ & $0(4)$ & $\mathrm{n} / \mathrm{a}$ & $0(8)$ \\
\cline { 2 - 6 } & MANUAL & $0(4)$ & $0(4)$ & $\mathrm{n} / \mathrm{a}$ & $0(8)$ \\
\cline { 2 - 6 } & Subtotal & $0(8)$ & $0(8)$ & $\mathrm{n} / \mathrm{a}$ & $0(16)$ \\
\hline \multirow{3}{*}{ TIME } & EFB & $1(4)$ & $0(4)$ & $\mathrm{n} / \mathrm{a}$ & $1(8)$ \\
\cline { 2 - 6 } & MANUAL & $0(4)$ & $0(4)$ & $\mathrm{n} / \mathrm{a}$ & $0(8)$ \\
\cline { 2 - 6 } & subtotal & $1(8)$ & $0(8)$ & $\mathrm{n} / \mathrm{a}$ & $1(16)$ \\
\hline & total & $1(16)$ & $0(16)$ & $\mathrm{n} / \mathrm{a}$ & $1(32)$ \\
\hline
\end{tabular}

TABLE D. 8

\begin{tabular}{|c|c|c|c|c|c|}
\hline & & TIME & FORCE & ERRORS & total \\
\hline \multirow{3}{*}{ LENGTH } & FEB & $0(4)$ & $0(4)$ & $\mathrm{n} / \mathrm{a}$ & $0(8)$ \\
\cline { 2 - 6 } & MANUAL & $0(4)$ & $0(4)$ & $\mathrm{n} / \mathrm{a}$ & $0(8)$ \\
\cline { 2 - 6 } & subtotal & $0(8)$ & $0(8)$ & $\mathrm{n} / \mathrm{a}$ & $0(16)$ \\
\hline \multirow{3}{*}{ TIME } & FEB & $1(4)$ & $0(4)$ & $\mathrm{n} / \mathrm{a}$ & $1(8)$ \\
\cline { 2 - 6 } & MANUAL & $0(4)$ & $0(4)$ & $\mathrm{n} / \mathrm{a}$ & $0(8)$ \\
\cline { 2 - 6 } & subtotal & $1(8)$ & $0(8)$ & $\mathrm{n} / \mathrm{a}$ & $1(16)$ \\
\hline & total & $1(16)$ & $0(16)$ & $\mathrm{n} / \mathrm{a}$ & $1(32)$ \\
\hline
\end{tabular}




\section{Appendix E}

\section{Typical Force/Torque Records for Additional Tasks}



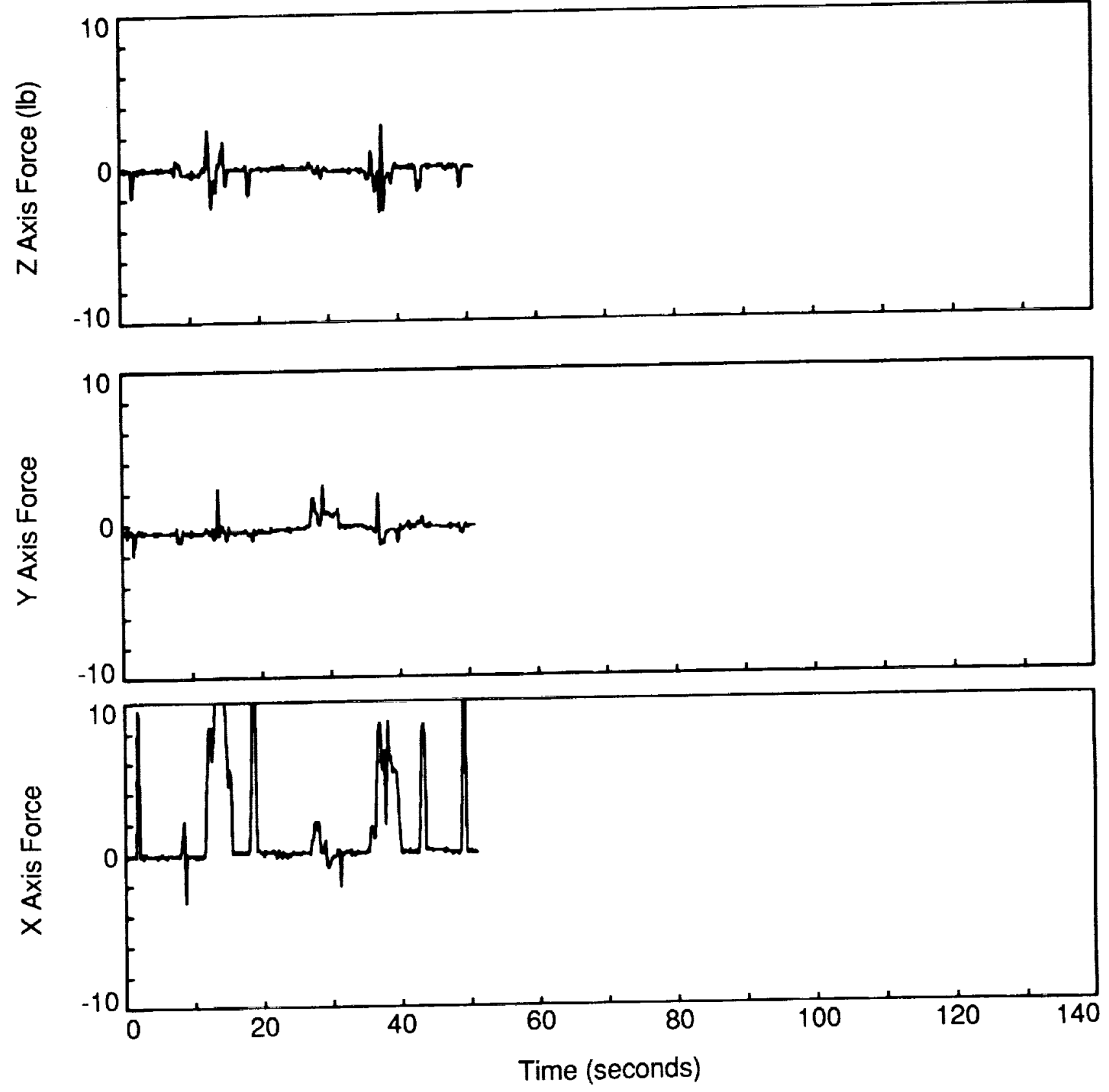

Figure E-1. Raw data acquired from a single repetition of the velcro experiment (continued in figures E-2 and E-3). $\mathrm{X}, \mathrm{Y}$, and $\mathrm{Z}$ axis forces measured in the robot hand reference frame. Spikes represent taps made intentionally by the operator to provide reference benchmarks in the force signal. 

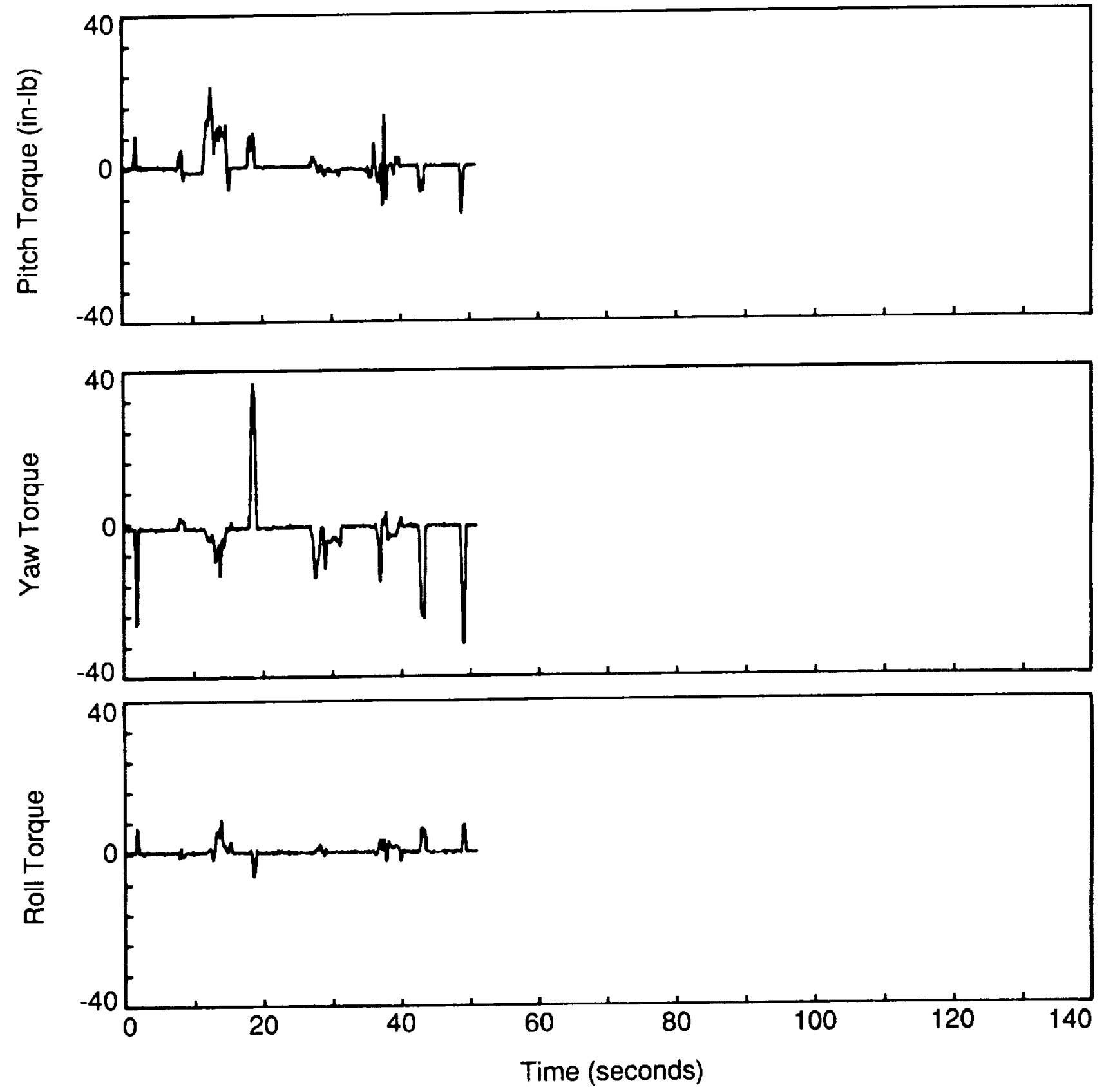

Figure E-2. Pitch, yaw and roll torques during the velcro task. 

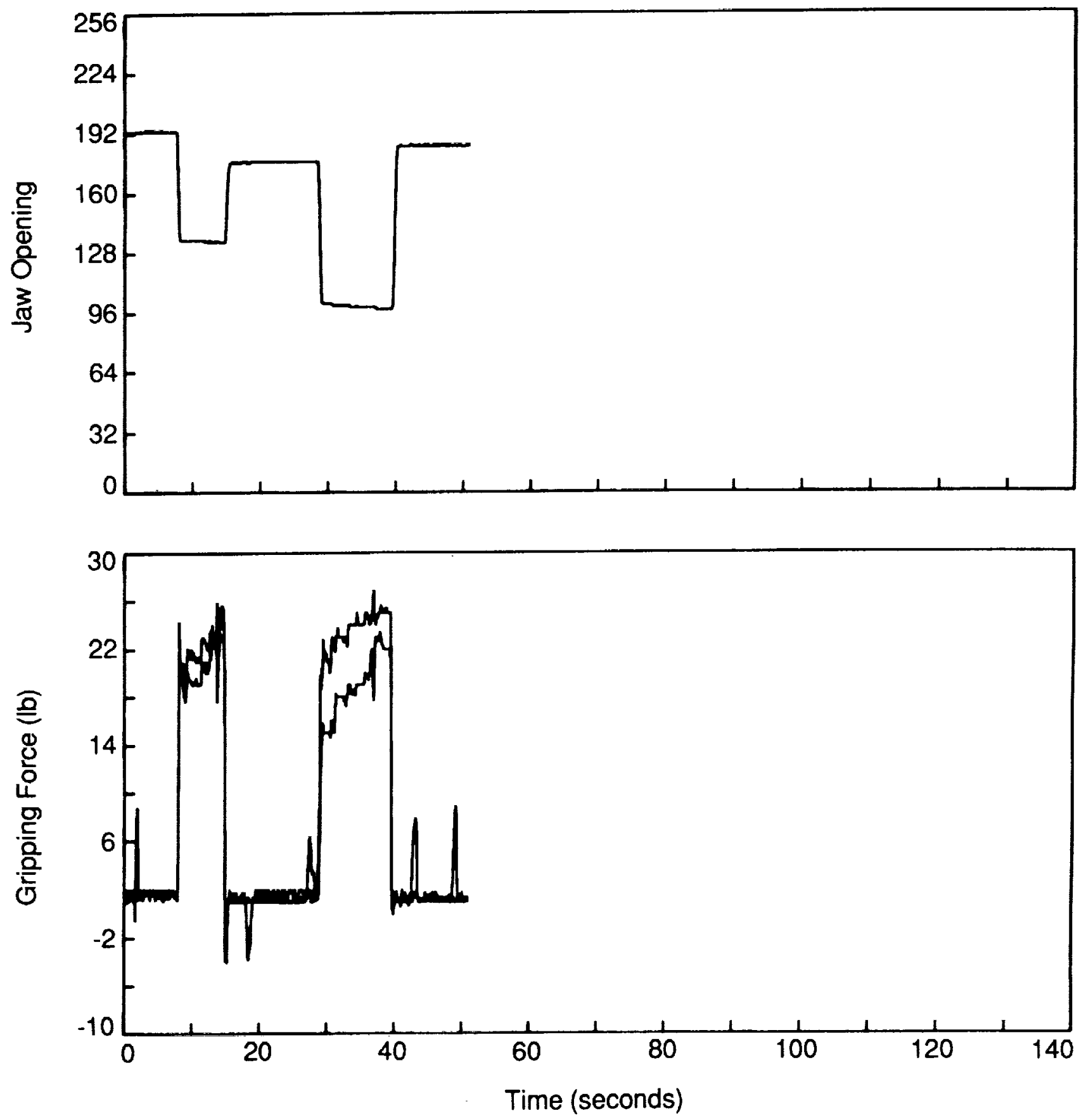

Figure E-3. Jaw opening and finger gripping force for the velcro task. 

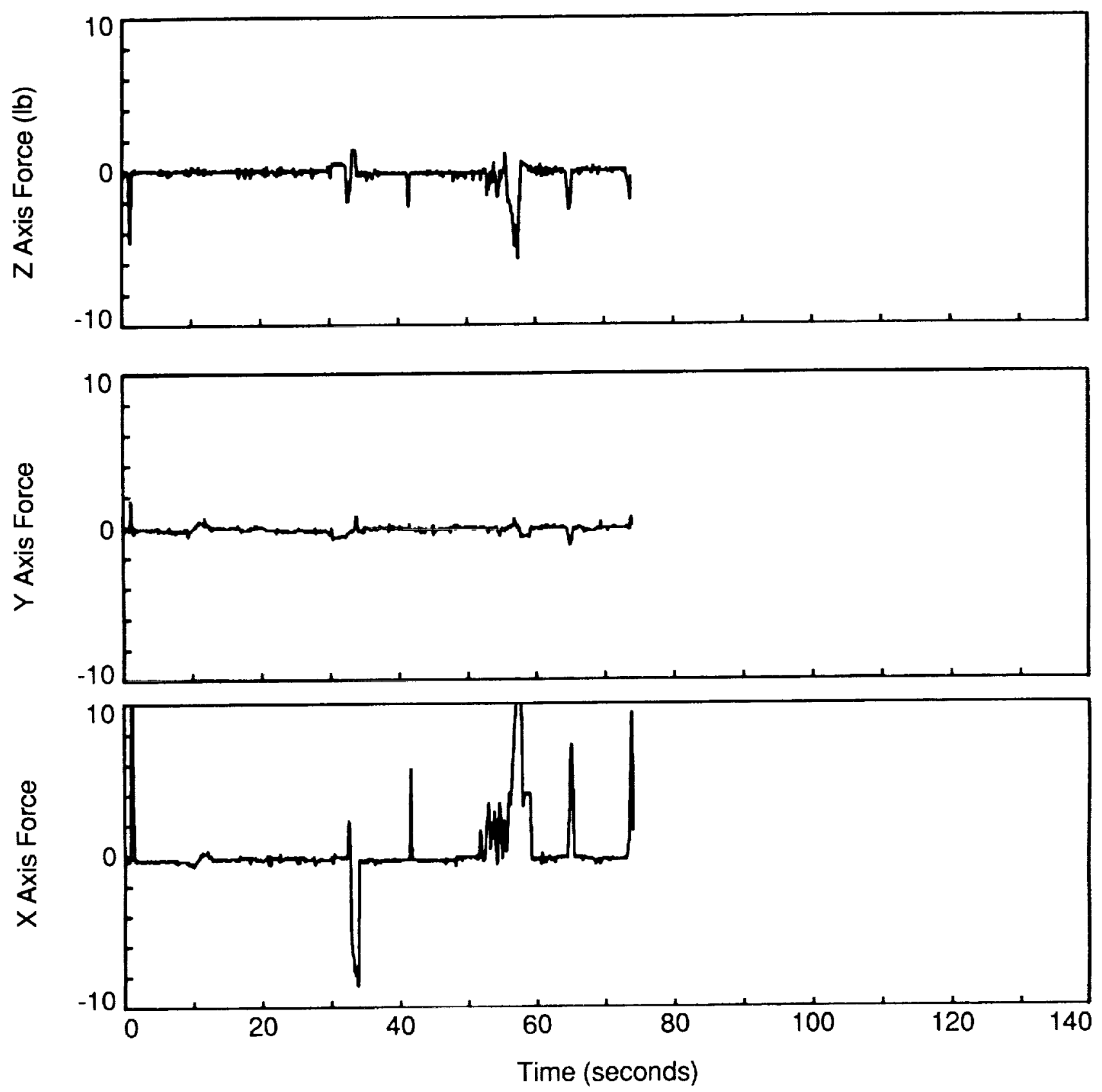

Figure E-4. Raw data acquired from a single repetition of the 25 pin connector experiment, continued in Figures E-5 and E-6. 

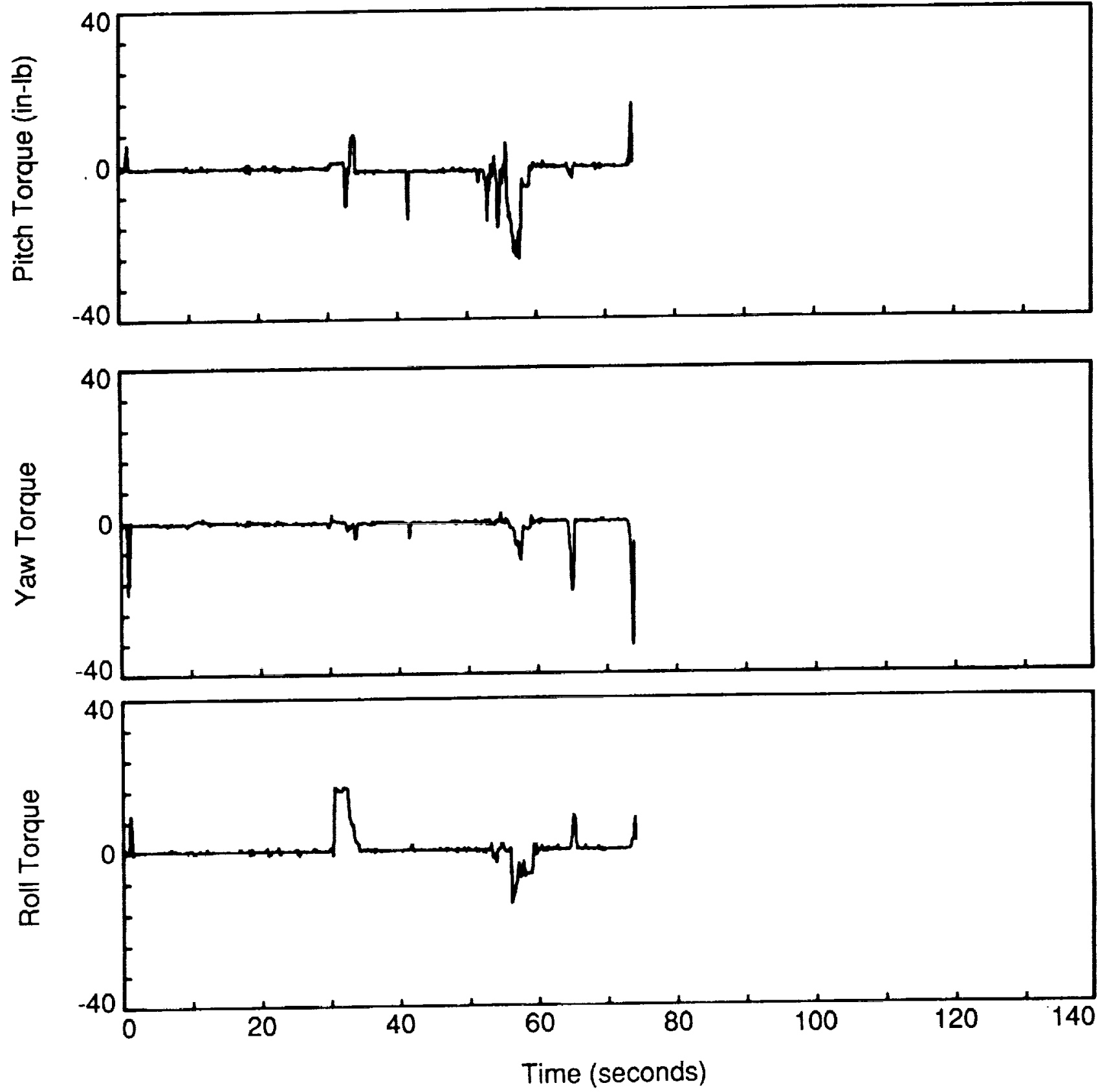

Figure E-5. Pitch, yaw and roll torques during the 25 pin connector task. 

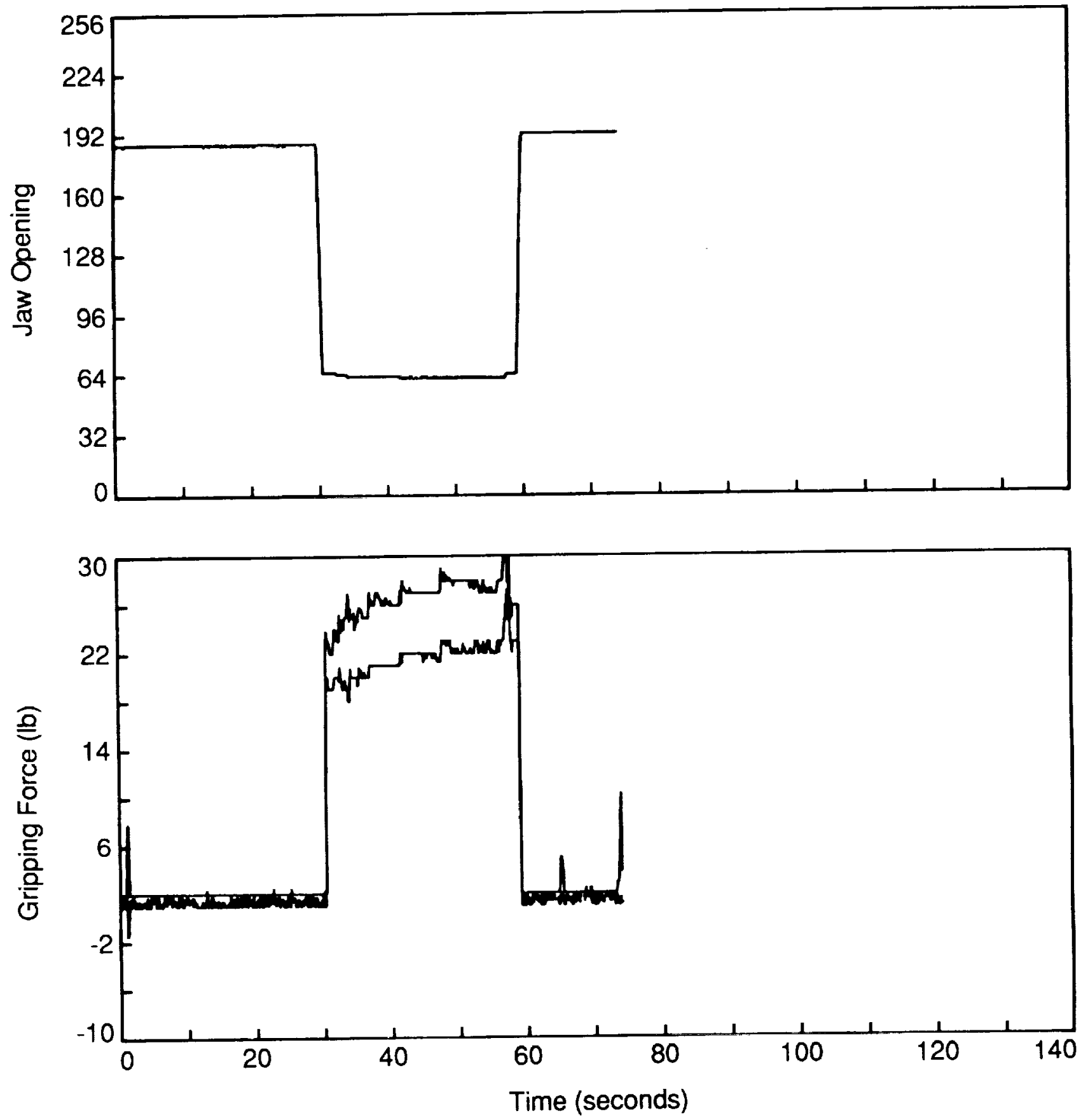

Figure E-6. Jaw opening and finger gripping force for the 25 pin connector task. 

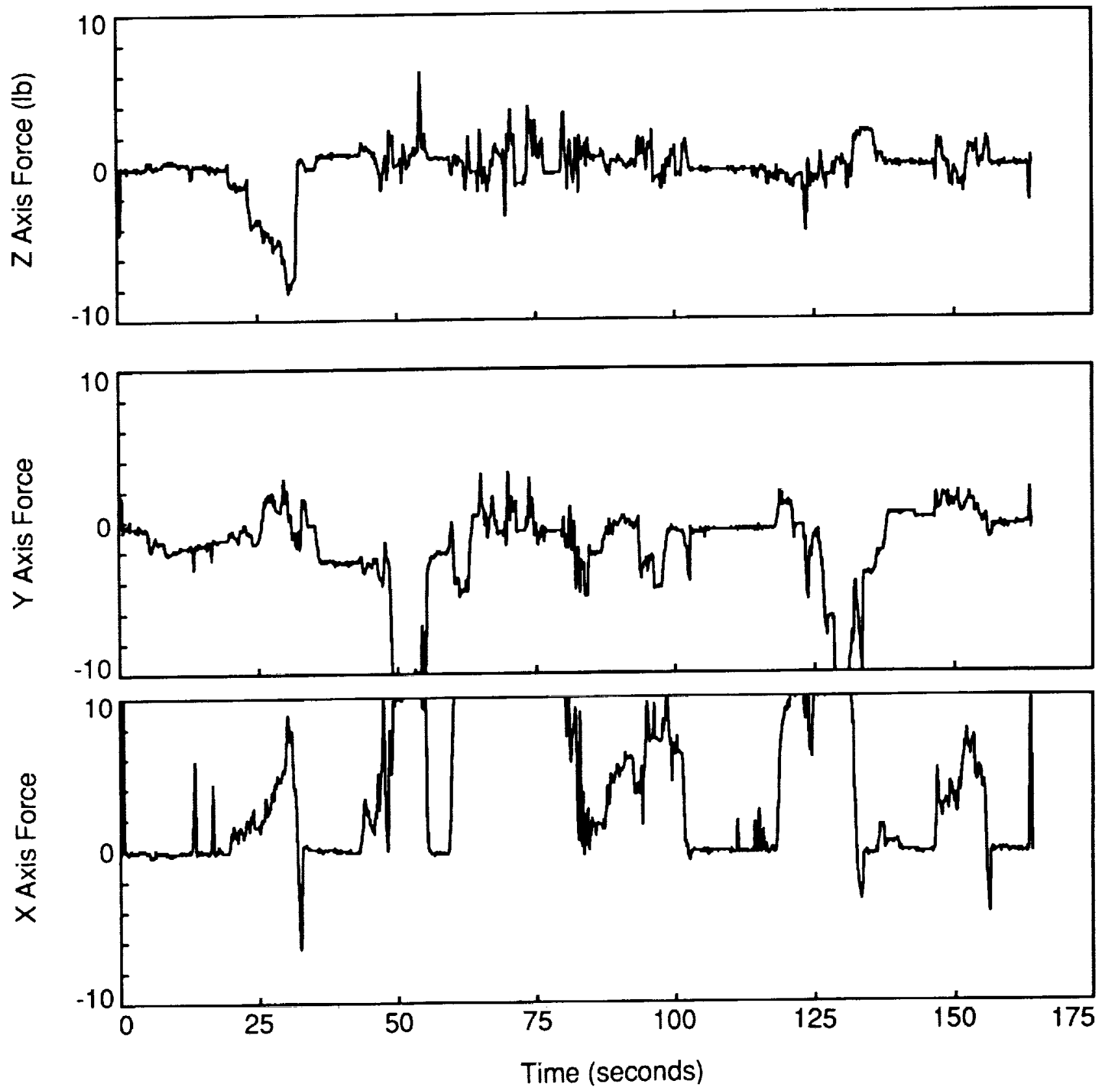

Figure E-7. Raw data acquired from a single repetition of the bayonet connector experiment, continued in figures E-8 and E-9. 

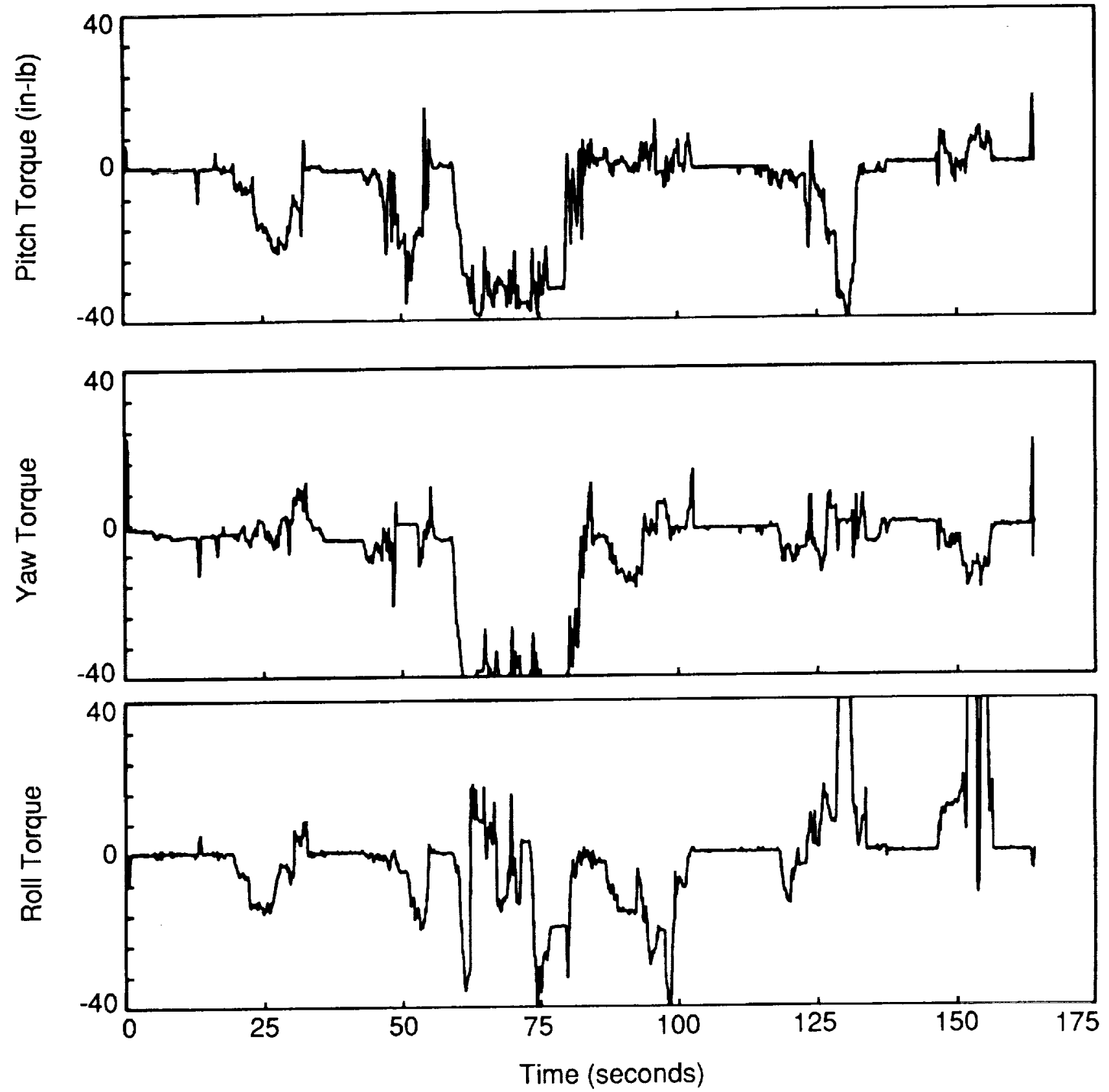

Figure E-8. Pitch, yaw and roll torques during the bayonet connector task. 

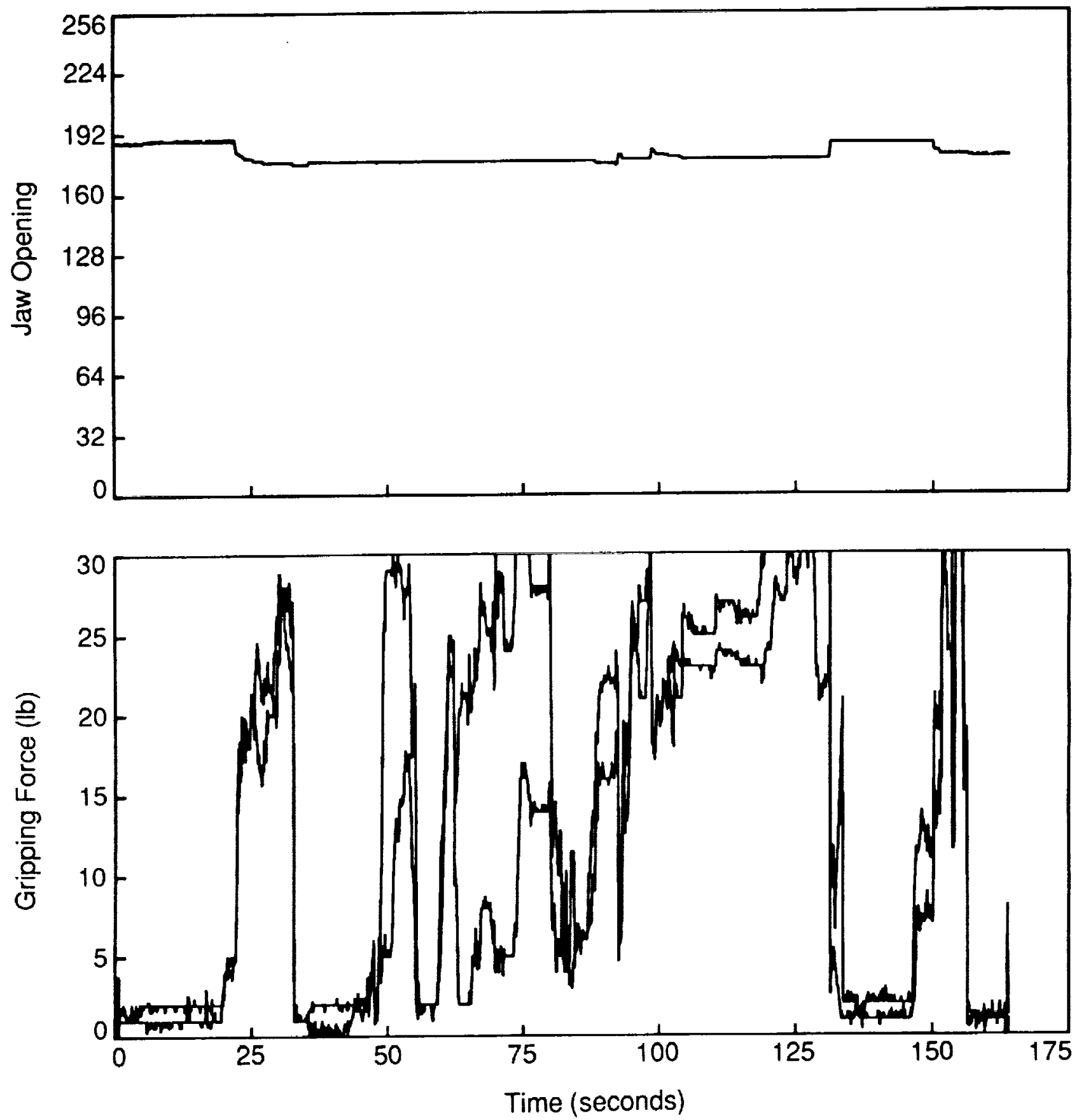

Figure E-9. Jaw opening and finger gripping force for the 25 pin connector task. 


\begin{tabular}{|c|c|c|}
\hline 1. Report No. & 2. Government Accession No. & 3. Recipient's Cotolog No. \\
\hline \multirow{2}{*}{\multicolumn{2}{|c|}{$\begin{array}{l}\text { 4. Title and Subtitle } \\
\qquad \begin{array}{l}\text { Performance Evaluation of a } \\
\text { Six-Axis Generalized Force- } \\
\text { RMXX Reflecting Teleoperator }\end{array}\end{array}$}} & 5. Report Date June 15, 1989 \\
\hline & & 6. Performing Organization Code \\
\hline \multicolumn{2}{|c|}{ 7. Author(s) B. Hannaford et al. } & 8. Performing Organization Report No. \\
\hline \multirow{3}{*}{\multicolumn{2}{|c|}{$\begin{array}{l}\text { 9. Performing Orgonization Nome ond Address } \\
\text { JET PROPULSION LABORATORY } \\
\text { California Institute of Technology } \\
4800 \text { Oak Grove Drive } \\
\text { Pasadena, California } 91109\end{array}$}} & 10. Work Unir No. \\
\hline & & $\begin{array}{l}\text { 11. Controct or Gront No. } \\
\text { NAS7 }-918\end{array}$ \\
\hline & & 13. Type of Report and Period Covered \\
\hline \multirow{2}{*}{\multicolumn{2}{|c|}{$\begin{array}{l}\text { 12. Sponsoring Agency Nome and Address } \\
\text { NATIONAL AERONAUTICS AND SPACE ADMINISTRATION } \\
\text { Washington, D.C. } 20546\end{array}$}} & \\
\hline & & 14. Sponsoring Agency Code \\
\hline
\end{tabular}

15. Supplementory Notes

16. Abstroct Recent Work at JPL in real-time distributed computation and control has culminated in a prototype force-reflecting telemanipulation system having dissimilar master (cable-driven force-reflecting hand controller) and slave (PUMA 560 robot with custom/controller), extremely high sampling rate $(1000 \mathrm{~Hz})$, and low loop computation delay $(5 \mathrm{msec})$. In a serles of experiments with this system and five trained test operators covering over 100 hours of teleoperation, we measured performance in a series of generic and application-driven tasks with and without force feedback, and with control shared between teleoperation and local sensor referenced control. Measurements defining task performance include $100-\mathrm{Hz}$ recording of six-axis force/torque information from the slave manipulator wrist, task completion time, and visual observation of predefined task errors.

The tasks consisted of high precision peg-in-hole insertion, electrical connectors, velcro attach-de-attach, and a twist-lock multi-pin connector. Each task was repeated three times under several operating conditions: "normal" bilateral telemanipulation, forward position control without force feedback, and shared control. In shared control, or lentation was locally servo controlled to comply with applied torques, while translation was under operator control. All performance measures improved as capability was added along a spectrum of capabilities ranging from pure position control through force-reflecting teleoperation and shared control. Performance was optimal for the bare-handed operator.

17. Key Words (Selected by Author(s)) Teleoperator, teleoperator performance, telemanipulation, force-reflecting telemanipulation system, six-axis generalized force-reflecting telemanipulation

\section{Distribution Statement}

Unclassified - Unlimited
19. Security Clossif. (of this roport) Unclassified
20. Security Clousif. (of this poge) Unclassif Ied
21. No. of Poges 130
22. Price 
-. 